\title{
Individualizing prostate cancer treatment
}

\author{
Citation for published version (APA):
}

van Wijk, J. H. (2021). Individualizing prostate cancer treatment: Development of treatment decision support systems for prostate cancer patients. [Doctoral Thesis, Maastricht University]. Maastricht University. https://doi.org/10.26481/dis.20211029jw

\section{Document status and date:}

Published: 01/01/2021

DOI:

10.26481/dis.20211029jw

Document Version:

Publisher's PDF, also known as Version of record

\section{Please check the document version of this publication:}

- A submitted manuscript is the version of the article upon submission and before peer-review. There can be important differences between the submitted version and the official published version of record.

People interested in the research are advised to contact the author for the final version of the publication, or visit the DOI to the publisher's website.

- The final author version and the galley proof are versions of the publication after peer review.

- The final published version features the final layout of the paper including the volume, issue and page numbers.

Link to publication

\footnotetext{
General rights rights.

- You may freely distribute the URL identifying the publication in the public portal. please follow below link for the End User Agreement:

www.umlib.nl/taverne-license

Take down policy

If you believe that this document breaches copyright please contact us at:

repository@maastrichtuniversity.nl

providing details and we will investigate your claim.
}

Copyright and moral rights for the publications made accessible in the public portal are retained by the authors and/or other copyright owners and it is a condition of accessing publications that users recognise and abide by the legal requirements associated with these

- Users may download and print one copy of any publication from the public portal for the purpose of private study or research.

- You may not further distribute the material or use it for any profit-making activity or commercial gain

If the publication is distributed under the terms of Article $25 \mathrm{fa}$ of the Dutch Copyright Act, indicated by the "Taverne" license above, 


\section{Individualizing prostate cancer treatment}

Development of treatment decision support systems for prostate cancer patients

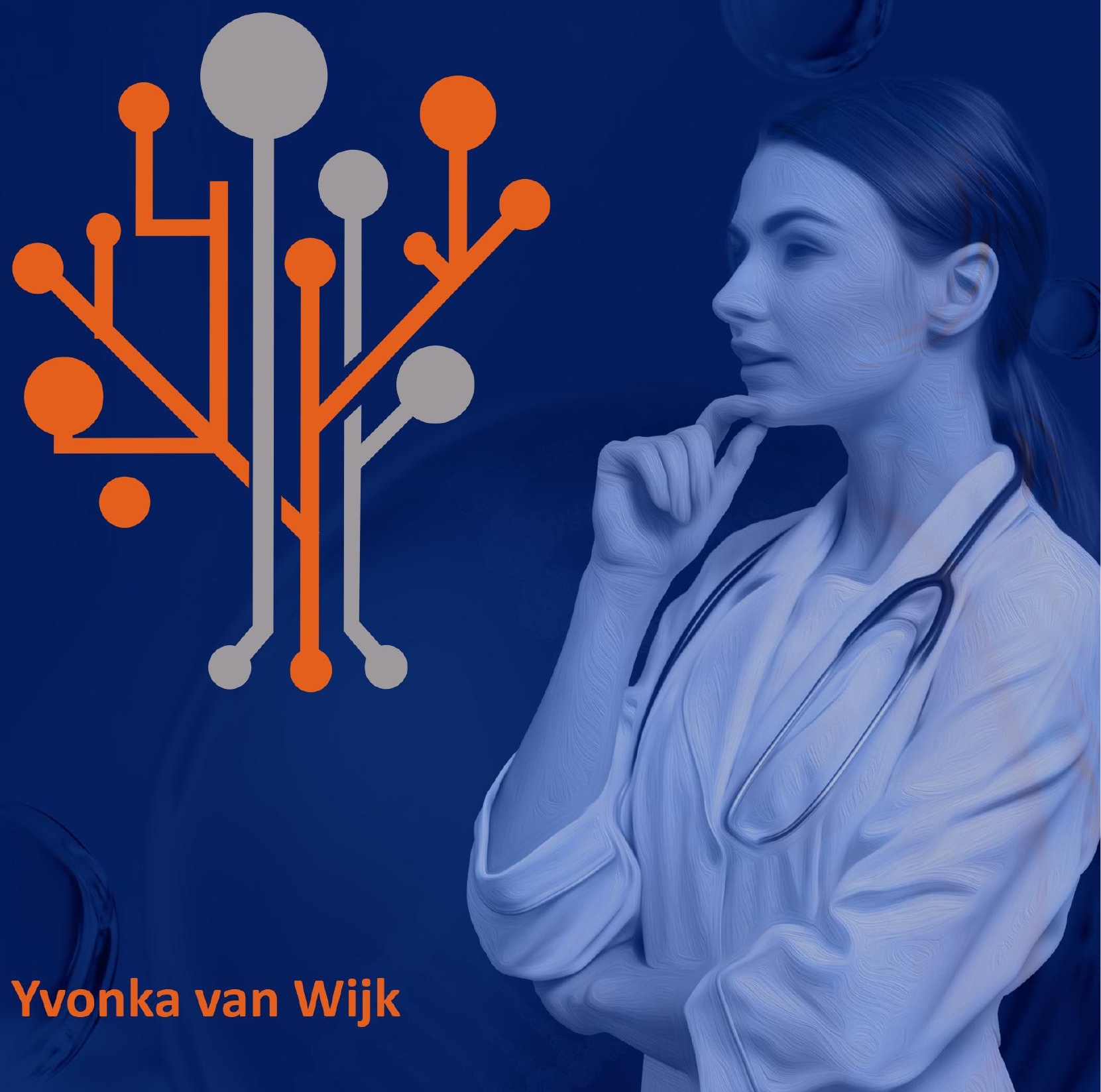




\section{Individualizing prostate cancer treatment}

Development of treatment decision support systems for prostate cancer patients 
Copyright @ Johanna Henrica van Wijk 2021

All rights reserved

No part of this publication may be reproduced, stored in a retrieval system or transmitted in any form or by any means, electronic, mechanical, photocopying, recording or otherwise, without prior permission of the author.

ISBN 978-94-6423-513-5

Printed by: $\quad$ ProefschriftMaken || www.proefschriftmaken.nl

Layout by: $\quad$ Tamara van Wijk

Cover design by: Tamara van Wijk 


\title{
Individualizing prostate cancer treatment
}

\author{
Development of treatment decision support \\ systems for prostate cancer patients
}

\section{Dissertation}

To obtain the degree of Doctor at the Maastricht University, on the authority of the Rector Magnificus Prof. Dr. Rianne M. Letschert, in accordance with the decision of the Board of Deans, to be defended in public on Friday 29th of October 2021 at 16:00 hrs

by 
Supervisor

Prof. Dr. P. Lambin

Co-supervisors

Dr. B.L.T. Ramaekers

Dr. B.G.L. Vanneste

Assessment Committee

Prof. Dr. Ir. F.J.W. Verhaegen (Chair)

Prof. Dr. D. Azria, University of Montpellier, France

Prof. Dr. M. van Vulpen, University of Technology Delft

Dr. B.F.M. Wijnen 


\section{Contents}

\section{General Introduction}

Chapter 1. Big data-based Decision Support systems for Radiotherapy

Chapter 2. Objective and Outline of the Thesis

Chapter 3. Decision support systems in prostate cancer treatment: an overview

\section{Treatment decision support systems for prostate cancer}

Chapter 4. Development of a virtual implantable rectum spacer as the basis for a decision support system

Chapter 5. Development of an isotoxic Decision Support System integrating genetic markers of toxicity for the implantation of a rectum spacer

Chapter 6. Influence of rectal spacer dynamics on anorectal dose and late rectal complication risk

Chapter 7. ProRaDS: Prostatectomy versus external beam Radiotherapy Decision Support system

\section{Discussion}

Chapter 8. General Discussion and future perspectives

\section{Appendices}

Summary

Impact Paragraph

Acknowledgements

Curriculum Vitae 

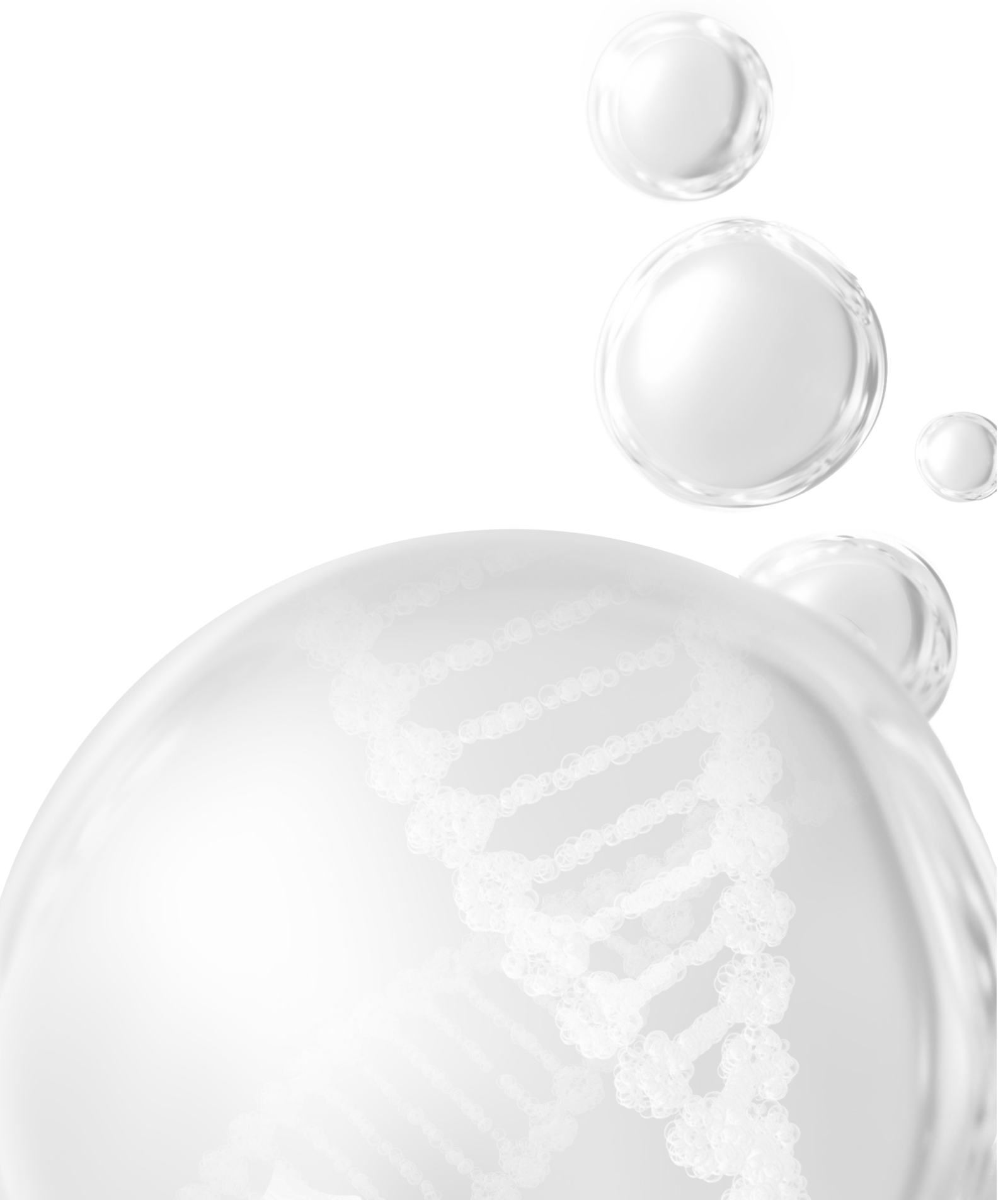
General Introduction 

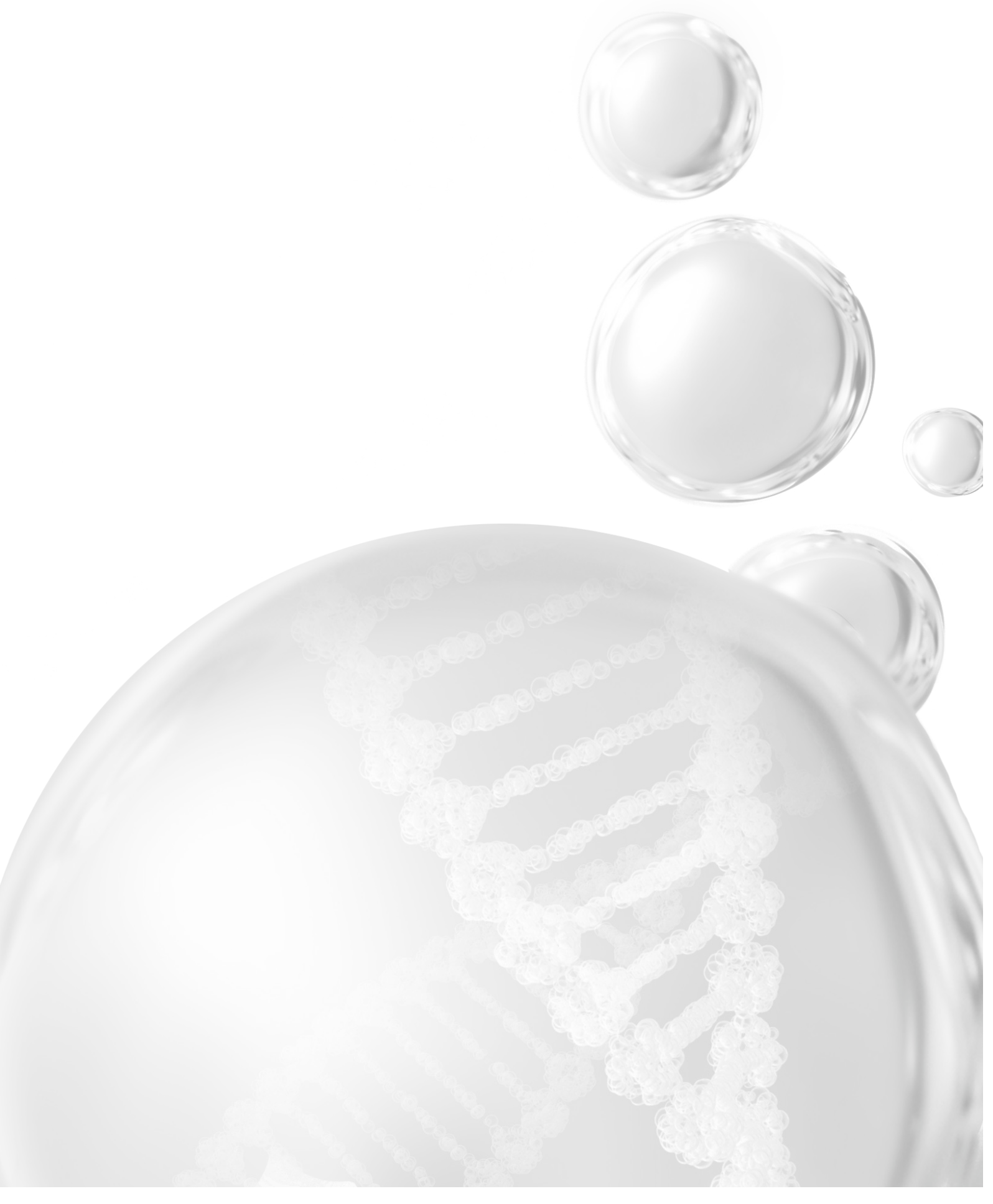


\section{Chapter 1}

\section{Big data-based Decision Support systems for Radiotherapy}

Authors:

Yvonka van Wijk, Cary Oberije, Erik Roelofs, Philippe Lambin

Adapted from:

"Big Data-Based Decision Support Systems for Hadron Therapy." Advances in Particle Therapy. CRC Press, 2018. 221-230

DOI: 10.1201/b22229 


\section{Introduction}

In the last decade, major advances have been made on the field of radiation oncology, bringing new diagnostic techniques and expanding the number of treatment modalities ${ }^{[1]}$. With a larger number of treatment options comes a heightened potential for personalized medicine, however, this has its challenges. Traditional evidence based medicine uses randomized trials that are designed to represent homogenous populations of patients, and are not based upon patient, disease and treatment parameters ${ }^{[2]}$.

The challenge in clinical decision making lies in that a physician must take into account a large number of characteristics, such as patient biology, pathology, medical images, blood test results, given medication, dose in the organs at risk, dose in the target volume, fractionation, and genomic data relevant to radiotherapy. The decisions should take into account the tumor control rate, the survival rate, and the radiotoxicity. The human cognitive capacity is limited however, making predictive modeling and big data in radiation oncology an increasingly essential tool in decision making ${ }^{[3]}$.

Particle therapy modalities, such as hadron therapy, are expected to be less toxic and more effective than the more conventional photon therapy, due to their favorable dose distributions ${ }^{[4,5]}$. However, planning studies show that not all patients would benefit from this more expensive treatment ${ }^{[6,7]}$, making decision support systems (DSS) an important tool to justify patient stratification for particle therapy.

This chapter will discuss the process of gathering data, training models and developing DSS using rapid learning health care (RLHC).

\section{Rapid Learning Healthcare}

During clinical trials, certain patient groups are under-represented, causing the data to be biased ${ }^{[8,9]}$. RLHC uses and reuses medical data from both clinical trials and from standard clinical practice to use in novel studies or for decision support in new patients ${ }^{[10,11]}$. RLHC consists of four sequential phases: the Data, Knowledge, Application, and Evaluation phase ${ }^{[3]}$. Figure 1.1 shows an overview of these phases. The first phase includes the obtaining and mining of prior data regarding the patients, disease, treatment and outcome. The second phase analyzes this data using methods such as machine learning to obtain knowledge from the data. The application phase improves clinical practice using this knowledge in the form of DSS. The final phase evaluates the DSS with regard to outcome, and uses this assessment as input for the first phase. 


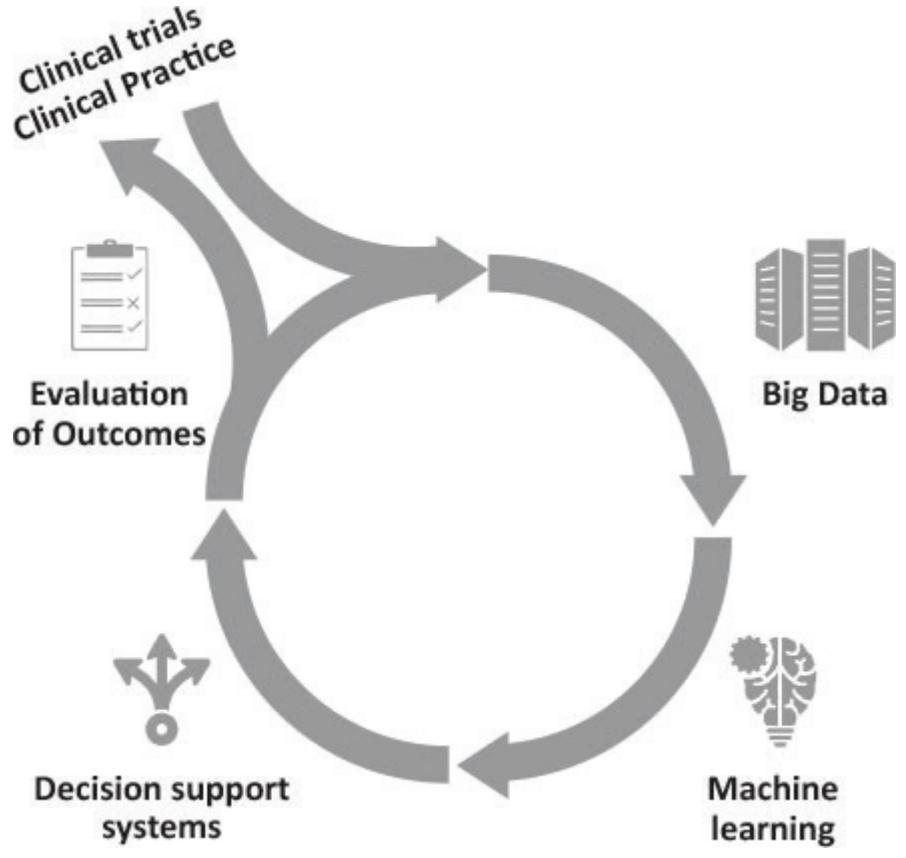

Figure 1.1: The phases of Rapid Learning Health Care (RLHC): Data collection from clinical practice and clinical trials, development of knowledge on the obtained data, application of the models via decision support systems (DSS), evaluation of the DSS and the application of DSS in clinical practice (Courtesy of T.P. van Wijk).

\section{Big data}

Ideally, the data obtained in the first phase of RLHC meets the classification demands of 'Big Data': the data should have Volume so that enhanced knowledge can be obtained with large amounts of variables; the data should have Velocity so that the gained knowledge remains practicable; the data should have Variety in order to find which treatment best fits an individual patient; and the data should have Veracity so that trust can be placed in the acquired knowledge ${ }^{[12]}$. The primary challenge in this phase of RLHC is obtaining data that meets these demands.

CancerLinQ (http://cancerlinq.org/) is an initiative to rise to this challenge by the American Society of Clinical Oncology (ASCO) $)^{[13]}$.

Figure 1.2 shows an overview of the CancerLinQ system. The system collects and analyzes all patient data obtained from the complete electronic health records (EHRs), and offers the opportunity to learn from previous patients, improving the quality of care. The system is built to transfer data including: patient and care provider demographics, appointments and visit details, medical and prescription history, surgery reports, pathology and lab data, and family history. Using language recognition methods, the software will even be able to extract information from clinician notes. 


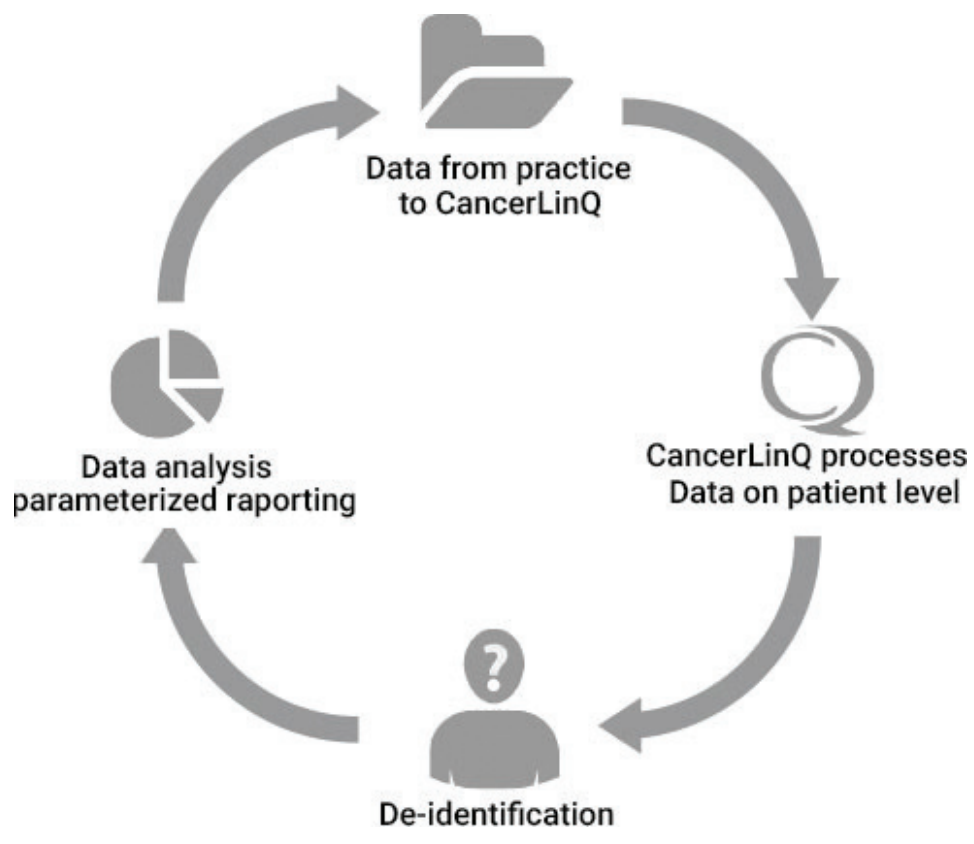

Figure 1.2: CancerLinQ is an initiative by the American Society of Clinical Oncology (ASCO) and works in four steps: data is uploaded to CancerLinQ from clinical practice, the data is processed on patient level, patient data is de-identified, the data is analyzed and reported and made available to clinical practice (Courtesy of T.P. van Wijk)

Centralization of data faces some difficult challenges, such as differences between institutes including language and data storage, and the reluctance to share data due to its value, privacy issues and lack of resources ${ }^{[14]}$. The first steps to overcoming these issues are being made through a cooperation between radiotherapy institutes all over the world (the Netherlands, Germany, Belgium, the UK, the USA, Italy, Denmark, Australia, India and China, with South Africa, Ireland and Canada as prospective partners), which applies RLHC using the euroCAT project (www.eurocat.info) ${ }^{[15]}$. Figure 1.3 shows the infrastructure of the euroCAT project. This project overcomes some of the classical barriers to data sharing by using 'distributed learning' to train RLHC models on data locally without the data needing to leave its relative institute (please see the animation: (https://youtu.be/ nQpqMluHyOk).

For this project to work, data within the institutes needs to be translated to general, well-defined terms. Machine-readable data needs to be developed, where local terms can be matched to the general ones, for example the $\mathrm{NCl}$ Thesaurus. These general terms serve as an interface for each institute, enabling 
information gathering and interpretation by a semantic gateway to the data. This also stimulates the harmonization between institutes on which data should be collected and how (disease specific 'umbrella' protocols ${ }^{[16]}$.

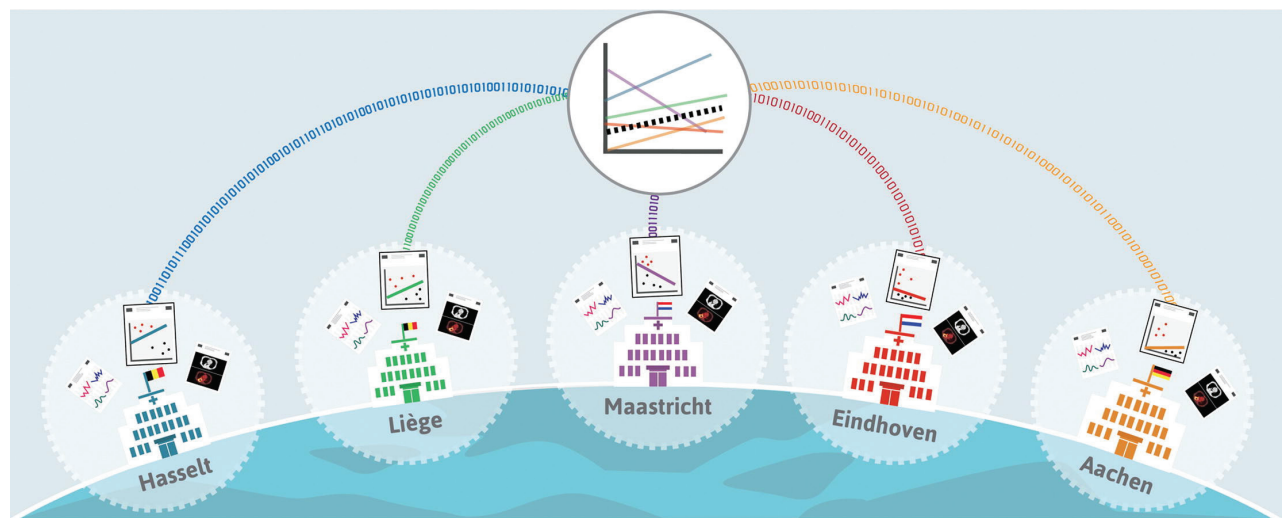

Figure 1.3: The graphical abstract for "Infrastructure and distributed learning methodology for privacy-preserving multi-centric rapid learning health care: euroCAT"[15].

Machine learning

Extracting knowledge from data is the third step in RLHC, and a common method used for this is machine learning ${ }^{[17]}$. Machine learning trains models on past data and uses it to make predictions regarding outcomes, such as tumor control and complication risks on data obtained from a new patient. Before models obtained with machine learning can be applied, they need to be validated, for which the TRIPOD (transparent reporting of a multivariable prediction model for individual prognosis or diagnosis) statement is a popular template ${ }^{[18]}$. The TRIPOD statement demands that the model is externally validated, so on dataset comparable to the training set, but from a different institute. There are a number of radiotherapy models available for different types of diseases, for example on https://www. ai4cancer.ai, https://mskcc.org, and http://research.nki.nl. 


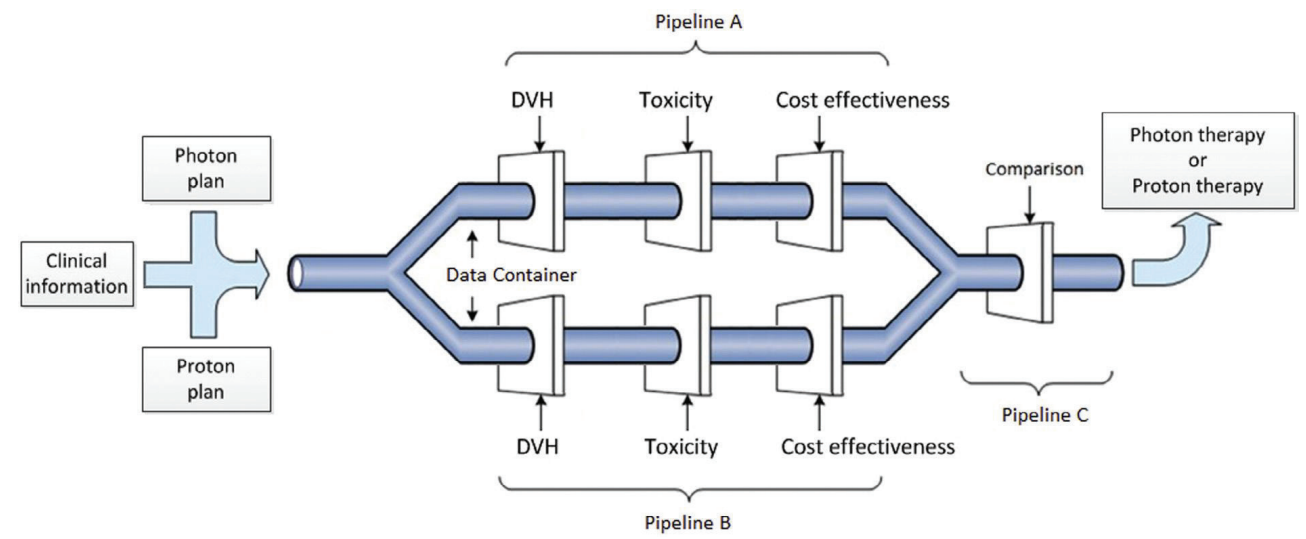

Figure 1.4: An example of a multifactorial Decision Support System that uses clinical information to make a three level comparison between proton and photon therapy for prostate cancer.

\section{Decision support systems}

The third phase of RLHC is the application of gained knowledge, which can be applied using DSS. DSS are typically software applications such as recursive partitioning analyses models, nomograms or websites such as https://www.ai4cancer.ai. A DSS is used to support the groups responsible for deciding on patient treatment, such as the physician, the tumor board and the patient, in making a knowledgeable decision regarding treatment options. Within the field of radiotherapy, it is acceptable to uses physics- and radiobiology-based models to predict complication risk and tumor control using tumor control probability (TCP) models ${ }^{[19]}$ and normal tissue complication probability models (NTCP) ${ }^{[20]}$. DSS is an extension to this practice, integrating validated physics and radiobiology based models, together with patient and disease parameters into a multifactorial structure ${ }^{[21]}$ (Figure 1.4).

The goal of a DSS is to use a combination of features (clinical, treatment, imaging, biomarkers, pathology, genetics etc.) and use it to make a robust prediction on patient outcome ${ }^{[22]}$, as shown in Figure 1.5. The outcome predicted by a DSS can include tumor control, including local recurrence or rate of metastases, complication probability, overall survival or even cost-effectiveness.

\section{Clinical features}

In several studies, clinical features have found to have a large influence on patient outcome ${ }^{[23,24]}$, and for a DSS to make individual predictions for patients, these features should be integrated into the system. Examples of clinical features are disease progression, organ function, age or sex of the patient, etc. The measurement of some of these features, such as performance status, is straightforward and can 
be done using methods such as questionnaires ${ }^{[25]}$. However, in order to reach a level of trustworthiness similar to that of randomized trials, the clinical parameters must be recorded in a meticulous manner. Standardized protocols should be used to this end, for example the 'Umbrella' protocol NCT01855191

\section{https://www.cancerdata.org/protocols/eurocat-umbrella-protocol-nsclc.}

\section{Multifactorial Decision Support Systems}

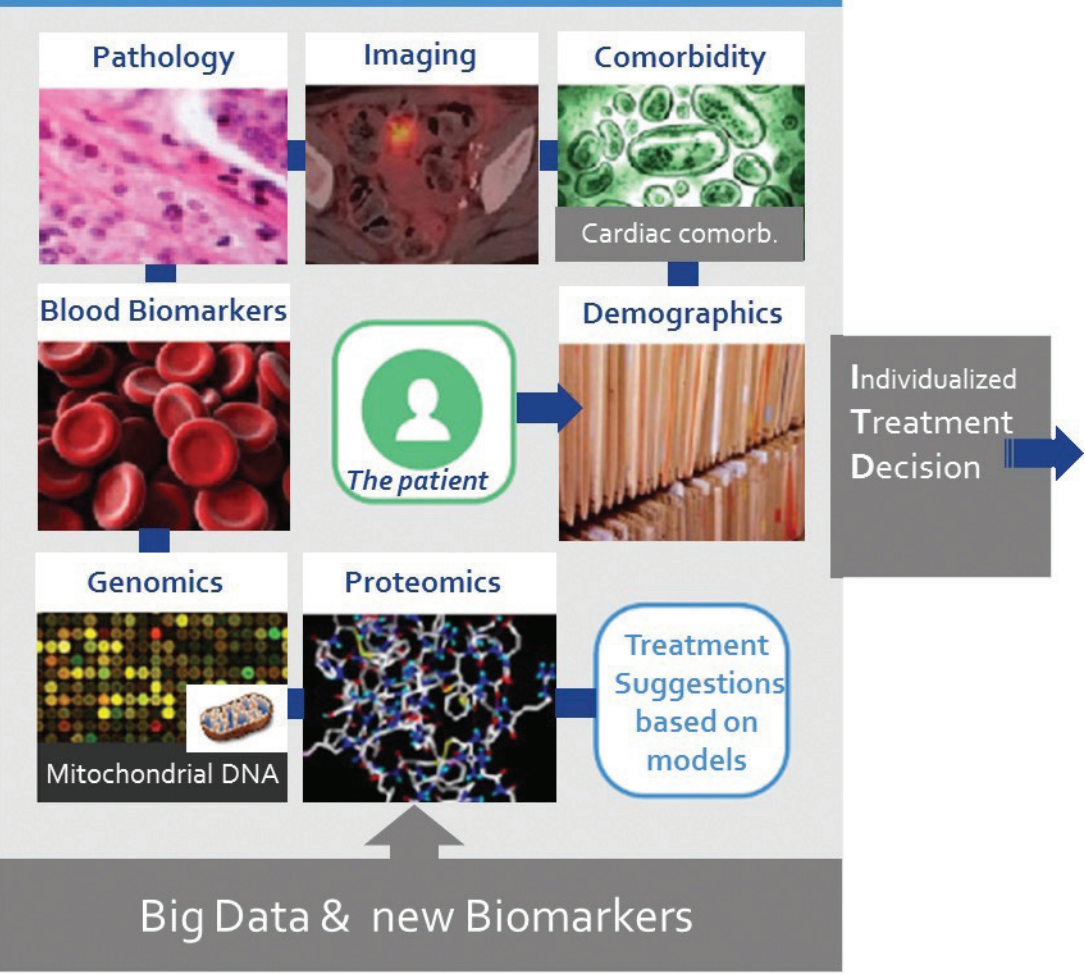

Figure 1.5: A multifactorial Decision Support System uses existing research evidences and resources to give a customized recommendation on patient treatment.

It is crucial that this is done after establishing a standardized protocol to ensure that the results are comparable between different centers and time points ${ }^{[26]}$. Additionally, perquisites for the measurements of certain features should be recorded, for example features evaluated only on patients showing certain symptoms, to prevent bias when interpreting the measurements.

Similar to the measurement of features, toxicity should be measured and 
graded using validated systems, for example the common terminology criteria for adverse events (CTCAE) ${ }^{[27]}$, which allows events to be reported by either patient or physician. A protocol must be established to report which system was used for observation and how events resulting from the treatment were managed. The reporting of the toxicity should be conducted in line with the Strengthening "The Reporting of Observational Studies in Epidemiology" (STROBE) statement for observational studies and genetic-association studies ${ }^{[28]}$. This statement is a protocol which lists all items that should be addressed during reporting to ensure standardized interpretation for these types of studies.

\section{Treatment features}

Radiotherapy treatment features include both dose features and additional, none radiation treatments given to the patient, such as chemotherapy or hormonal therapy, which have shown to influence the outcome. Modern technologies such as intensity modulated radiotherapy (IMRT), brachytherapy, volumetric arc radiotherapy (VMAT) or particle-beam therapy, such as hadron therapy, allow for localized dose delivery around the target volume with maximum sparing of the organs at risk with very high dosimetric accuracy ${ }^{[29]}$. These techniques, in combination with increasing knowledge of normal tissue response to radiotherapy ${ }^{[30]}$, is used to keep the delicate balance between TCP and NTCP. Figure 1.6 shows the dose distribution resulting from IMRT, VMAT, intensity modulated proton therapy (IMPT) and brachytherapy (BT) treatment planning on the prostate.

To model the dose response in a DSS, the relevant features are obtained from the planned dose distributions. To ensure that these features are realistic, measures should be taken to deliver the prescribed dose as planned and these measures should be described ${ }^{[31]}$. One of the modelling challenges on this field is how best to combine the dose distribution for every sub-volume of a structure into features. Parameters such as mean dose, maximum dose, volume receiving more than a set amount of dose, or equivalent uniform dose (EUD) are easily quantified and are often used, which is acceptable for a large range of applications. However, to improve personalized decision making, spatial characteristics should be analyzed as well to differentiate in radio-sensitivity in different regions of organ or tumor ${ }^{[32]}$. 


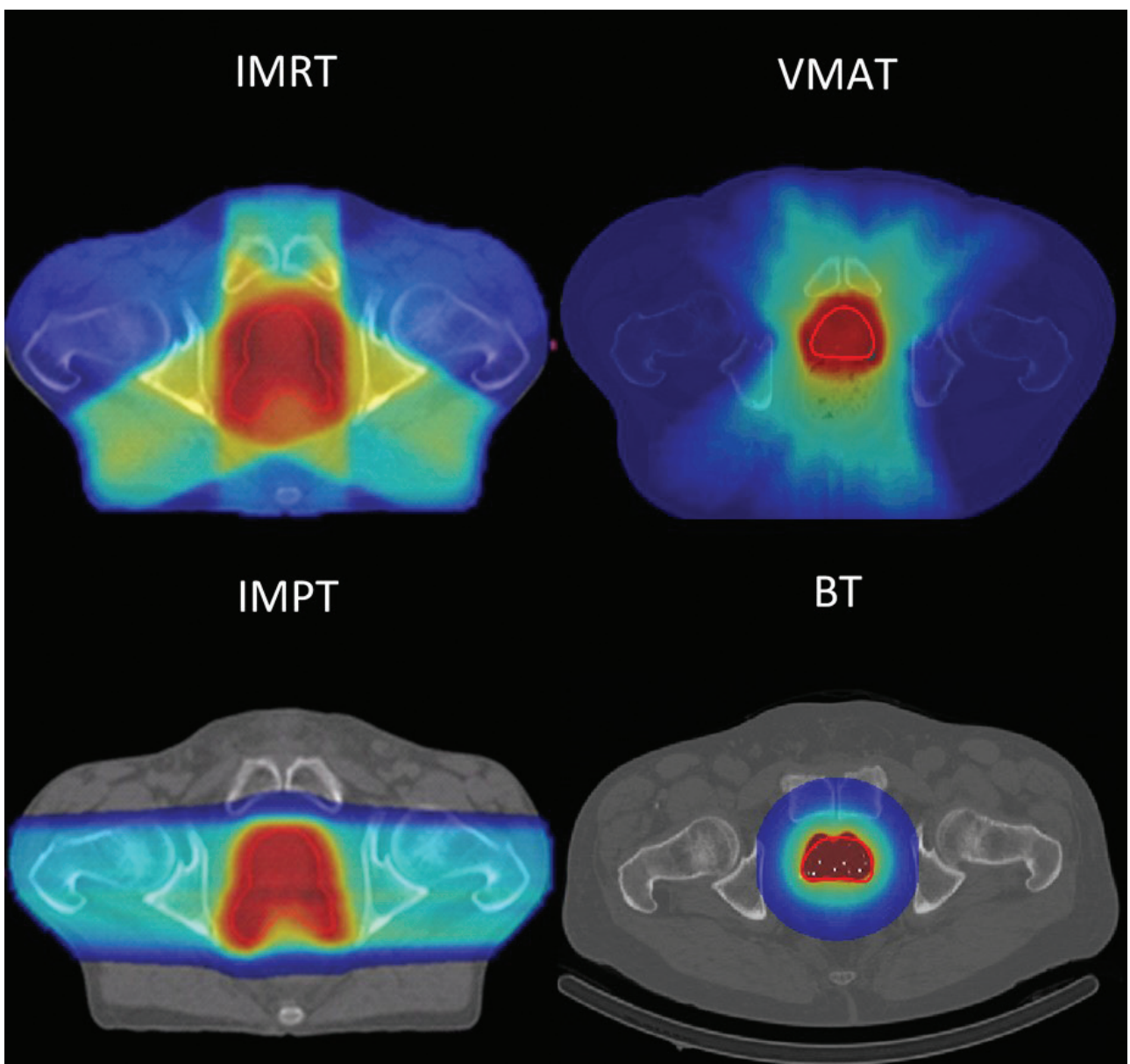

Figure 1.6: Dose distributions resulting from intensity modulated radiotherapy (IMRT), volumetric arc radiotherapy (VMAT), intensity modulated proton therapy (IMPT) and brachytherapy (BT) treatment planning on a patient with prostate cancer.

\section{Cost-effectiveness}

In the past, the endpoint of most DSS have been TCP and/or NTCP. However, due to the limited resources available in healthcare and considering the increasing costs of newer treatments, cost-effectiveness (CE) comparisons are becoming increasingly relevant ${ }^{[33]}$. This is especially important when considering treatments which are not significantly beneficial to all patients, but are notably more expensive or invasive than traditional treatments, such as hadron therapy ${ }^{[34]}$. A CE analyses is a good way to weigh the TCP, the NTCP and the treatment costs against each other. Additionally, a CE analyses could improve the insurance perspective regarding more expensive treatments, and could allow reimbursement for patients for whom such a treatment proves cost-effective ${ }^{[35]}$. 


\section{Evaluation}

The goal of RLHC is to improve predictability of treatment outcomes to allow increase of effectiveness and efficiency of patient treatment. However, it is important to verify the improved predictability of multifactorial decision support systems compared to generally accepted, and often less complex, models ${ }^{[36]}$. Accepted RLHC models should repeatedly be reevaluated using robust data to test if the outcome is as expected and how this relates to evidence based guideline knowledge.

\section{Summary}

Modern healthcare aspires to optimize personalized cancer therapy and faces many challenges in this respect. The increase in available treatment options and the diversity in patients prove incredibly problematic for individualized decision making. However, DSS developed using RLHC have the potential to bring us one step closer to realizing that goal. An essential step that needs to be taken is the standardization of data acquisition, including data concerning treatment, clinical features, imaging, genetics, and outcome. Also the clinical assessment of developed DSS is critical, as well as standardizing the development of robust prediction models. 


\section{References}

1. Vogelzang, N.J., et al., Clinical cancer advances 2011: Annual Report on Progress Against Cancer from the American Society of Clinical Oncology. J Clin Oncol, 2012. 30(1): p. 88-109.

2. Maitland, M.L. and R.L. Schilsky, Clinical trials in the era of personalized oncology. CA Cancer J Clin, 2011. 61(6): p. 365-81.

3. Abernethy, A.P., et al., Rapid-learning system for cancer care. J Clin Oncol, 2010. 28(27): p. 4268-74.

4. Grutters, J.P., et al., When to wait for more evidence? Real options analysis in proton therapy. Oncologist, 2011. 16(12): p. 1752-61.

5. Pijls-Johannesma, M., et al., Particle therapy in lung cancer: where do we stand? Cancer Treat Rev, 2008. 34(3): p. 259-67.

6. Roelofs, E., et al., Design of and technical challenges involved in a framework for multicentric radiotherapy treatment planning studies. Radiother Oncol, 2010. 97(3): p. 567-71.

7. van der Laan, H.P., et al., The potential of intensity-modulated proton radiotherapy to reduce swallowing dysfunction in the treatment of head and neck cancer: A planning comparative study. Acta Oncol, 2013. 52(3): p. 561-9.

8. Grand, M.M. and P.C. O'Brien, Obstacles to participation in randomised cancer clinical trials: a systematic review of the literature. J Med Imaging Radiat Oncol, 2012. 56(1): p. 31-9.

9. Murthy, V.H., H.M. Krumholz, and C.P. Gross, Participation in cancer clinical trials: race-, sex-, and agebased disparities. JAMA, 2004. 291(22): p. 2720-6.

10. Dehing-Oberije, C., et al., Development and external validation of prognostic model for 2-year survival of non-small-cell lung cancer patients treated with chemoradiotherapy. Int J Radiat Oncol Biol Phys, 2009. 74(2): p. 355-62.

11. Roelofs, E., et al., Results of a multicentric in silico clinical trial (ROCOCO): comparing radiotherapy with photons and protons for non-small cell lung cancer. J Thorac Oncol, 2012. 7(1): p. 165-76.

12. Lustberg, T., et al., Big Data in radiation therapy: challenges and opportunities. Br J Radiol, 2017. 90(1069): p. 20160689.

13. Schilsky, R.L., et al., Building a rapid learning health care system for oncology: the regulatory framework of CancerLinQ. J Clin Oncol, 2014. 32(22): p. 2373-9.

14. Budin-Ljosne, I., et al., DataSHIELD: an ethically robust solution to multiple-site individual-level data analysis. Public Health Genomics, 2015. 18(2): p. 87-96.

15. Deist, T.M., et al., Infrastructure and distributed learning methodology for privacy-preserving multicentric rapid learning health care: euroCAT. Clinical and Translational Radiation Oncology, 2017. 4: p. 24-31.

16. Meldolesi, E., et al., An umbrella protocol for standardized data collection (SDC) in rectal cancer: a prospective uniform naming and procedure convention to support personalized medicine. Radiother Oncol, 2014. 112(1): p. 59-62.

17. Kourou, K., et al., Machine learning applications in cancer prognosis and prediction. Comput Struct Biotechnol J, 2015. 13: p. 8-17.

18. Collins, G.S., et al., Transparent Reporting of a multivariable prediction model for Individual Prognosis or Diagnosis (TRIPOD): the TRIPOD statement. Ann Intern Med, 2015. 162(1): p. 55-63. 
19. Walsh, S. and W. van der Putten, A TCP model for external beam treatment of intermediate-risk prostate cancer. Med Phys, 2013. 40(3): p. 031709.

20. Michalski, J.M., et al., Radiation dose-volume effects in radiation-induced rectal injury. Int J Radiat Oncol Biol Phys, 2010. 76(3 Suppl): p. S123-9.

21. Dekker, A., et al., Rapid learning in practice: a lung cancer survival decision support system in routine patient care data. Radiother Oncol, 2014. 113(1): p. 47-53.

22. Bright, T.J., et al., Effect of clinical decision-support systems: a systematic review. Ann Intern Med, 2012. 157(1): p. 29-43.

23. Klopp, A.H. and P.J. Eifel, Biological predictors of cervical cancer response to radiation therapy. Semin Radiat Oncol, 2012. 22(2): p. 143-50.

24. Kristiansen, G., Diagnostic and prognostic molecular biomarkers for prostate cancer. Histopathology, 2012. 60(1): p. 125-41.

25. Schmidt, M.E. and K. Steindorf, Statistical methods for the validation of questionnaires--discrepancy between theory and practice. Methods Inf Med, 2006. 45(4): p. 409-13.

26. Garrido-Laguna, I., et al., Validation of the Royal Marsden Hospital prognostic score in patients treated in the Phase I Clinical Trials Program at the MD Anderson Cancer Center. Cancer, 2012. 118(5): p. 1422-8.

27. National Cancer Institute (U.S.), Common terminology criteria for adverse events (CTCAE). Rev. ed. NIH publication. 2009, Bethesda, Md.: U.S. Dept. of Health and Human Services, National Institutes of Health, National Cancer Institute. $194 \mathrm{p}$.

28. von Elm, E., et al., The Strengthening the Reporting of Observational Studies in Epidemiology (STROBE) Statement: guidelines for reporting observational studies. Int J Surg, 2014. 12(12): p. 1495-9.

29. Jaffray, D.A., Image-guided radiotherapy: from current concept to future perspectives. Nat Rev Clin Oncol, 2012. 9(12): p. 688-99.

30. Jaffray, D.A., et al., Accurate accumulation of dose for improved understanding of radiation effects in normal tissue. Int J Radiat Oncol Biol Phys, 2010. 76(3 Suppl): p. S135-9.

31. Hermans, B.C., et al., Weekly kilovoltage cone-beam computed tomography for detection of dose discrepancies during (chemo)radiotherapy for head and neck cancer. Acta Oncol, 2015. 54(9): p. 1483-9.

32. Bentzen, S.M., Theragnostic imaging for radiation oncology: dose-painting by numbers. Lancet Oncol, 2005. 6(2): p. 112-7.

33. Amin, N.P., D.J. Sher, and A.A. Konski, Systematic review of the cost effectiveness of radiation therapy for prostate cancer from 2003 to 2013. Appl Health Econ Health Policy, 2014. 12(4): p. 391-408.

34. Ramaekers, B.L., et al., Protons in head-and-neck cancer: bridging the gap of evidence. Int J Radiat Oncol Biol Phys, 2013. 85(5): p. 1282-8.

35. Sensible and sustainable care W.a.S. the Council for Public Health and Health Care to the Minister of Health, Editor. 2006: Zoetermeer.

36. Steyerberg, E.W., et al., Assessing the performance of prediction models: a framework for traditional and novel measures. Epidemiology, 2010. 21(1): p. 128-38. 


\section{Chapter 2}

Objective and Outline of the Thesis 


\section{Objectives}

The focus of this work was on the third phase of the rapid learning healthcare cycle: the development of clinical decision support systems (DSS). The primary aim of this thesis was the following:

"Bridging the gap between machine learning based models and the decision making process through the development of clinical decision support systems for prostate cancer patients"

We focused our efforts on prostate cancer patients, as this disease has a large variety of viable treatment options with similar cure rates, and a large demographic that could benefit from such a tool ( 2,400 patients in the Netherlands receive active treatment for prostate cancer per year). We divided our primary aim into two sub-objectives:

Objective 1: To provide an overview of decision support systems in prostate cancer treatment

Objective 2: To develop and challenge new clinical decision support systems that aid in the treatment selection of prostate cancer both a) within the field of radiotherapy and b) multidisciplinary.

\section{Hypotheses}

Relating to Objective 2, we tested the following hypotheses:

1. Image deformation can be used to create a CT scan of a patient with an implantable rectum spacer prior to implantation, allowing for dose comparisons.

2. A clinical decision support system can be used to identify patients for whom an implantable rectum spacer is beneficial prior to treatment.

3. The expected biochemical failure-free survival of patients can be improved when using an iso-toxic DSS to decide on the implantation of an implantable rectum spacer during external beam radiotherapy.

4. The expected quality of life and cost-effectiveness of patients can be improved when using a clinical decision support system to decide between external beam radiotherapy and prostatectomy for the treatment of prostate cancer.

Thesis outline

In this work we focus on the development of DSS for prostate cancer treatment, particularly external beam radiotherapy (EBRT). We split this thesis into 3 parts: Part I consist of the general introduction and a literature overview to work towards meeting Objective 1; Part II consists of the development and of new DSS which addresses Objective 2; Part III consists of a general discussion (Figure 2.2) . 
Part I

To demonstrate the significance of rapid learning health care, and the role of DSS in this practice, we provide a general introduction in Chapter 1.

The first step to meeting our primary aim and addressing Objective 1 was to gain insight in current literature concerning DSS for prostate cancer. In order to identify the gap in current decision support systems regarding prostate cancer treatment, an overview is made of current available tools, including diagnostic support tools, treatment DSS, and patient decision aids (PDA) in Chapter $\mathbf{3}$.

Part II

Objective $2 a$ was to develop a new DSS within the field of radiotherapy. During EBRT for prostate cancer the anterior rectal wall, which lays in close proximity to the prostate, has the risk to receive high dose levels, possibly leading to chronic rectal bleeding. In order to reduce the dose in this organ at risk, rectal spacers have been developed, such as the implantable rectum spacer (IRS) (Figure 2.1). Chapters 4-6 cover the development, refinement and challenge the robustness of a proof of concept DSS for the placement of an IRS during EBRT treatment of prostate cancer. Chapter 4 describes the development of a virtual IRS (V-IRS) which uses image deformation to generate virtual CT image of a patient with IRS. This V-IRS is used as the basis for a new three-level DSS based on the V-IRS, comparing dose, NTCP and TCP on both the image without IRS and with V-IRS. The performance of this V-IRS is assessed by comparing to the CT images of real IRS'. Chapter $\mathbf{5}$ expands this DSS by making it iso-toxic, thus calculating the maximum achievable TCP possible for a given patient based on toxicity restraints on the rectum, as well as by including two SNPs predictive for late rectal bleeding. In Chapter $\mathbf{6}$ we challenged the robustness of the V-IRS by testing the effect of the exact volume of the IRS on dose metrics and NTCP values calculated on the rectum. This study used a set of patients who had received a balloon spacer at the beginning of treatment, and for whom cone-beam CTs were collected in the course of treatment. During this time, the balloon spacer deflates, reducing the space gained by the IRS for each image. We compared the dose metrics in the rectum and the NTCP values for late rectal bleeding of the later images to the treatment planning image. 


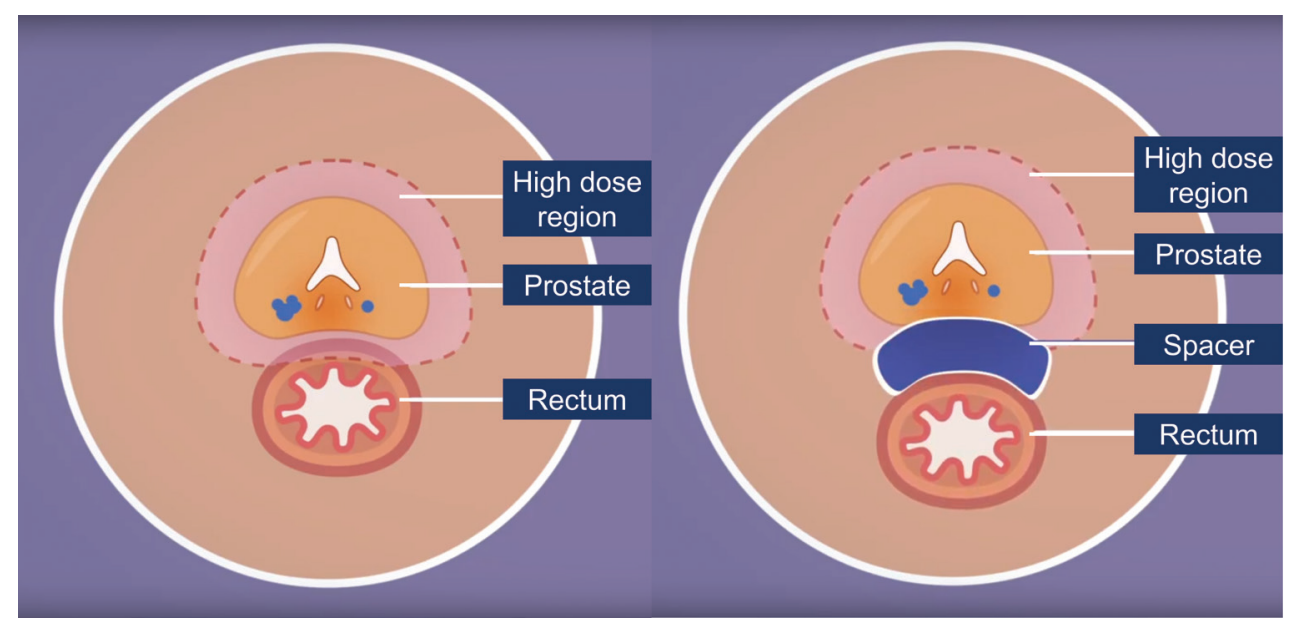

Figure 2.1 - Graphic overview of prostate anatomy during radiotherapy and the effect of the implantable rectum spacer

Objective $2 b$ was to develop and challenge a new DSS on a multidisciplinary level. In Chapter $\mathbf{7}$ we developed a DSS for treatment using radical prostatectomy (RP) or EBRT. This was done by combining a set of predictive models for TCP and probability of late rectal bleeding, erectile dysfunction and urinary incontinence with an extensive cost-effectiveness analysis. In this chapter we tested our DSS using a synthetic patient dataset, which used a combination of published clinical parameters to create a representation of low-intermediate risk prostate cancer patients. The synthetic dataset was adjusted to recreate published clinical trials for the validation of the DSS. We also used this synthetic dataset to create a proof-ofconcept in vitro clinical trial.

\section{Part III}

In the final section a critical discussion is presented of how well the objectives were met, and in how far the obtained knowledge through this research has filled the gap in the literature. As a supplement to this reflection, further research aspects are suggested that would further the development and integration of DSS for prostate cancer research. This section also discusses developments and challenges of individualized patient decision aids (iPDAs), which forms an important field parallel to DSS to further shared decision making. 


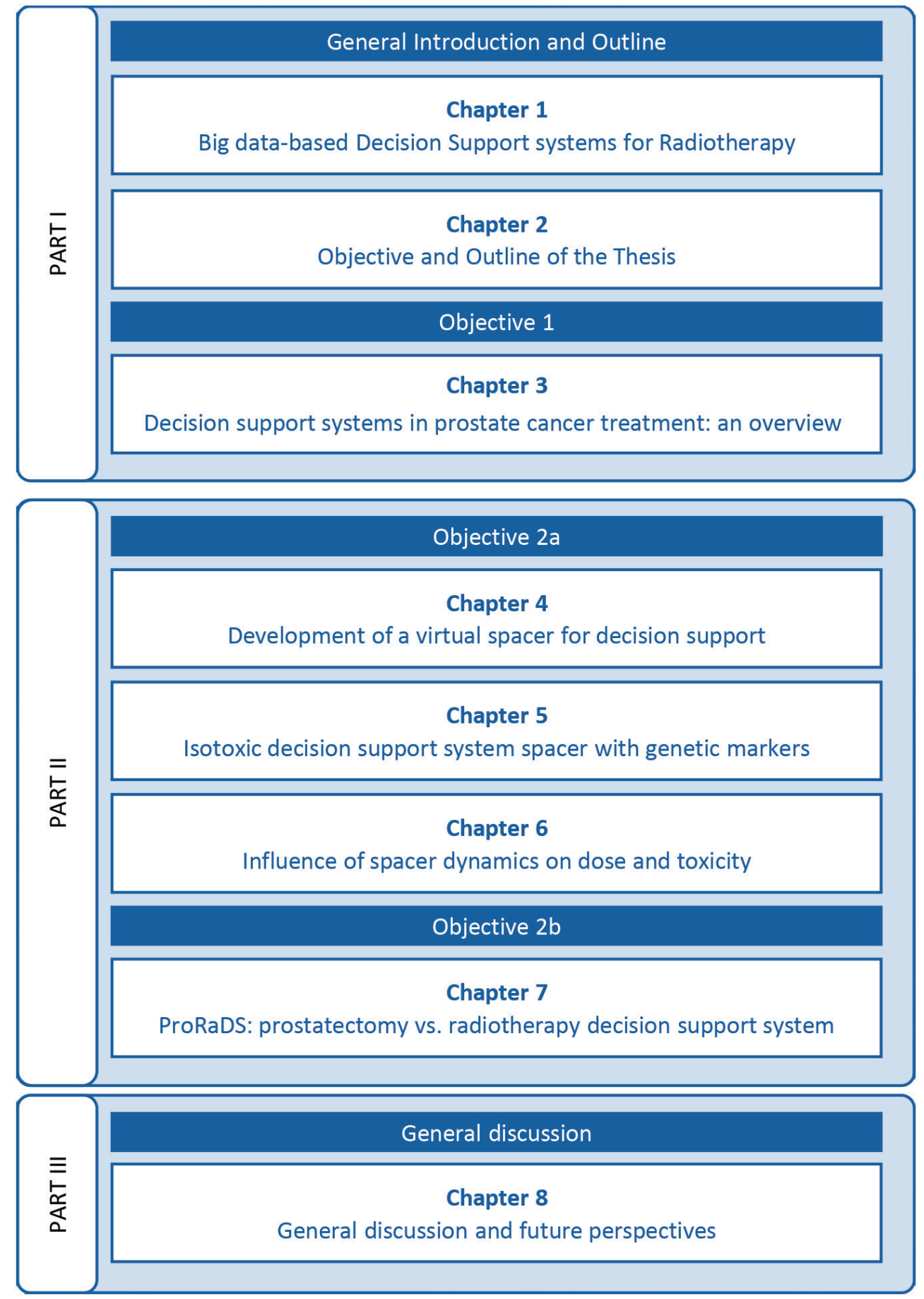

Figure 2.2-Outline of the thesis 

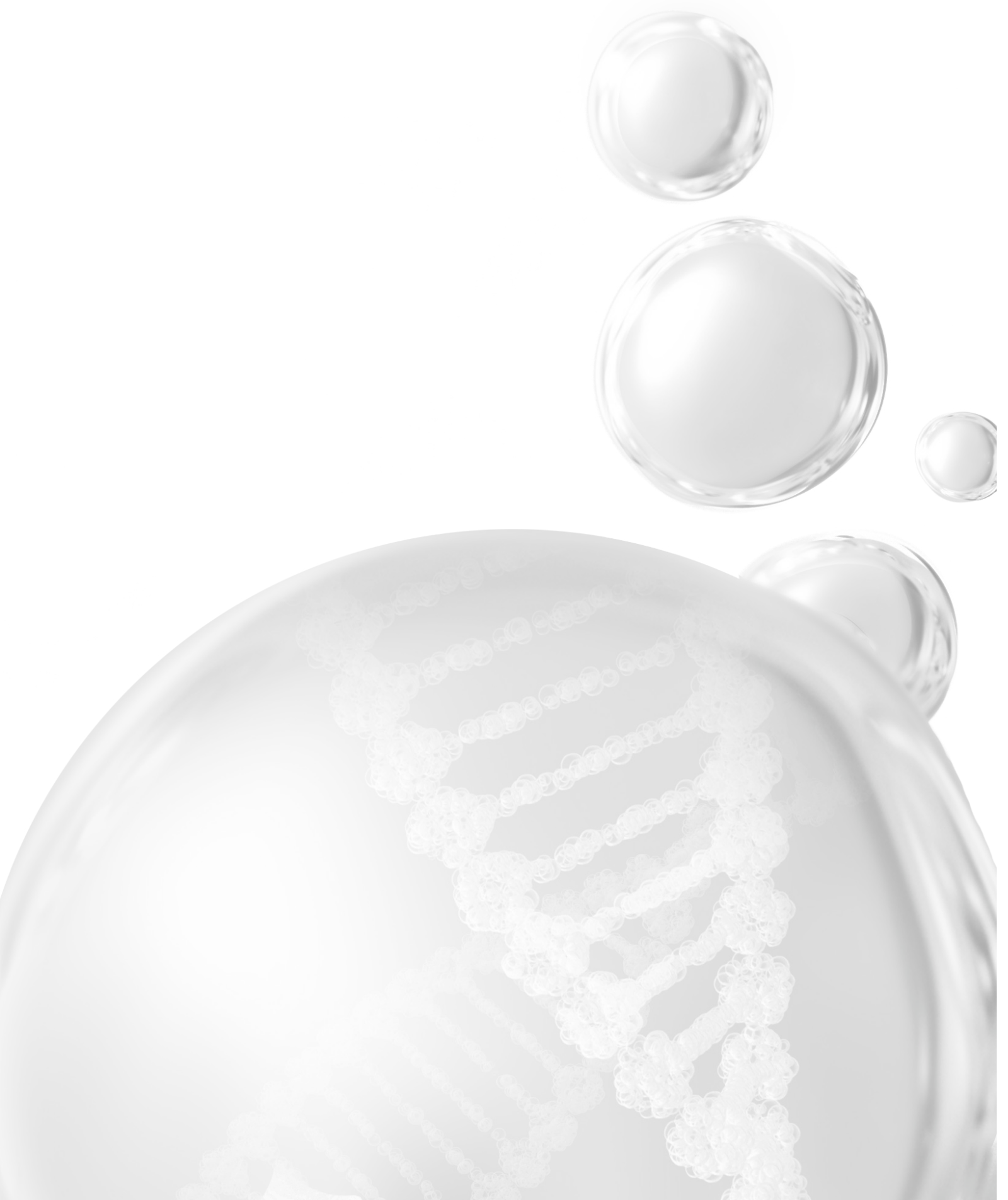


\section{Chapter 3}

\section{Decision support systems in prostate cancer treatment: an overview}

Authors:

Yvonka van Wijk, Iva Halilaj, Evert van Limbergen, Sean Walsh, Ludy Lutgens, Philippe Lambin, Ben Vanneste

Adapted from:

"Decision Support Systems in Prostate Cancer Treatment: An Overview." BioMed research international 2019 (2019)

DOI: $10.1155 / 2019 / 4961768$ 


\begin{abstract}
Background: A multifactorial decision support system (DSS) is a tool designed to improve the clinical decision-making process, while using clinical inputs for an individual patient to generate case-specific advice. The study provides an overview of the literature to analyze current available DSS focused on prostate cancer (PCa), in order to better understand the availability of decision support tools as well as where the current literature is lacking.
\end{abstract}

Methods: We performed a MEDLINE literature search in July 2018. We divided the included studies into different sections: diagnostic, which support the diagnosis of PCa; treatment, which support the decision between treatment modalities or treatment plans; and patient, which focusses on informing the patient. We manually screened and excluded studies that did not contain a DSS concerning prostate cancer and study proposals.

Results: Our search resulted in twelve diagnostic DSS; six treatment DSS; two patient DSS; and eight papers that could improve DSS.

Conclusions: Diagnosis DSS is well represented in the literature as well as treatment DSS considering external beam radiotherapy, however, there is a lack of DSS for other treatment modalities. The development of patient decision aids is a new field of study, and few successes have been made for PCa patients. These tools can improve personalized medicine, but need to overcome a number of difficulties to be successful and require more research. 


\section{Introduction}

Worldwide, prostate cancer (PCa) is the second most occurring type of cancer in men, and the most commonly diagnosed cancer for men living in developed countries, making it a very relevant topic for cancer research ${ }^{[1]}$.

A variety of treatment options is available to treat PCa, such as externalbeam radiotherapy (EBRT) and radical prostatectomy ${ }^{[2-4]}$, which have similar long-term survival outcomes. Other treatments, such as brachytherapy ${ }^{[5-8]}$ are gaining popularity, and active surveillance is an increasingly viable option as well ${ }^{[9,}$ 10], due to the slow progression of some kinds of PCa. Retrospective studies comparing different treatment modalities tend to be conflicting and biased. Consensus on the best treatment choices for men with PCa remains absent because prospective trials for different treatments report different toxicities ${ }^{[4}$, ${ }^{11,12]}$. Due to this, the treatment choice is largely dependent on both patient and physician subjective preferences, rather than knowledge-based decisionmaking ${ }^{[13]}$. Additionally, treatment outcome is dependent on a large number of features, including treatment, patient, tumor, clinical, and genetic features ${ }^{[14]}$. These factors further complicate the integration of evidence-based decision making into clinical practice due to the limitations of human cognitive capacity, which can only take a relatively small number of factors into account on which to base a decision ${ }^{[15,16]}$. In order to meet the aspiration of personalized medicine, the need for multifactorial decision support systems (DSS) is growing ${ }^{[17-23]}$. A DSS is a tool designed to improve the difficult medical decision-making process. It uses multifactorial inputs (treatment, patient, tumor, clinical, genetic, etc.) for a given patient to generate case-specific advice for patients, clinicians or other medical professionals. Due to the variety of treatment options for PCa, all equally efficacious for outcome, but having different secondary effects, this disease is an interesting subject for the use of DSS.

In addition to the need for DSS for treatment selection, similar systems can be used for the diagnosis of PCa, improving early detection as well as reducing over-diagnosis and unnecessary testing. These DSS can use imaging, clinical, biological, and other parameters to improve detection and risk classification of PCa in a minimally invasive method to maximize individual treatment.

This study provides an overview of the literature to analyze current available DSS focused on PCa, in order to better understand the availability of DSS as well as noting where the current literature is lacking. We aim to provide an update for clinicians about recent advances in DSS for personalized PCa oncology, which may improve clinical decision-making. 


\section{Materials and Methods}

\section{Search Strategy}

In order to identify all decision support systems with relations to treatment for PCa, we performed a MEDLINE/PubMed literature search in July 2018, restricted to English. Details of the strategy we used are shown in Figure 3.1.

\section{Search terms:}

Patient:

- Prostate

- Cancer, tumour, tumor, carcino* or oncol*

Outcome:

- Toxicity, ntcp, adevers* or complica*

- Outcome, tcp, prdict*, survival or failure

Design:

- Decision support system, clinical decision support system or multifactorial decision support system

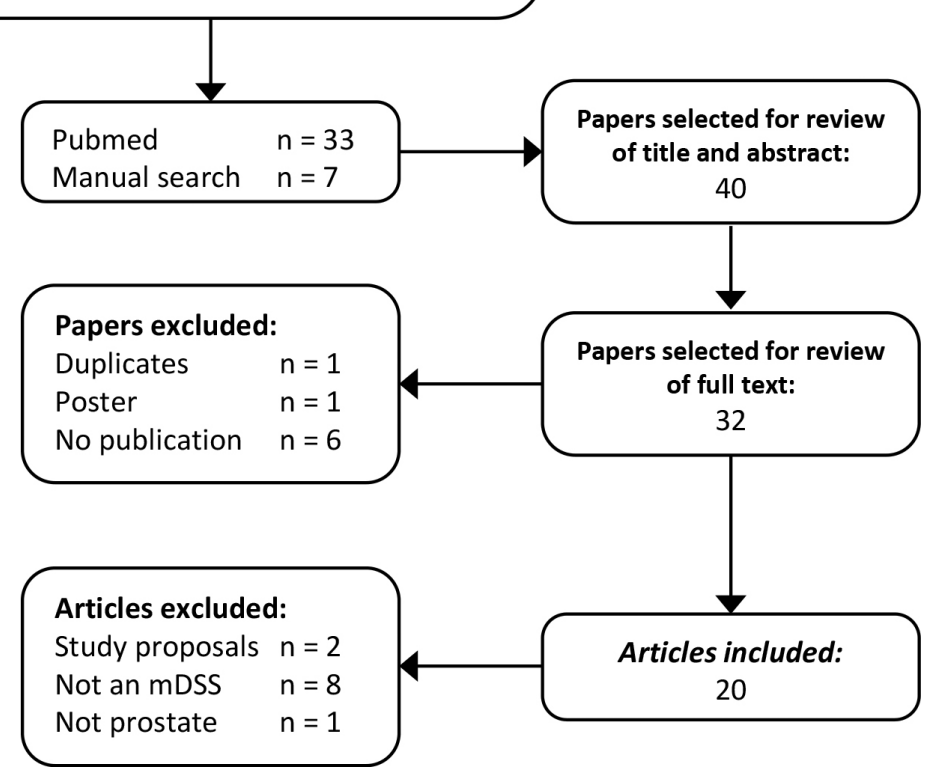

Figure 3.1 - Flowchart of the results of the literature search in PubMed

\section{Selection criteria}

Prior to review full texts, we manually checked the abstracts and titles to select papers for this study. Duplicates, posters or abstracts that did not include a published work were excluded. Additionally, we excluded studies which clearly did not include a DSS for PCa. After full text review, we excluded any papers that described study proposals that did not describe a DSS in PCa. We selected appropriate studies by manually screening and considering the aforementioned criteria. 


\section{Study characteristics}

The studies we included were divided into sections according to the type of DSS: diagnostic DSS, which support the staging of PCa or support the decision for more invasive or expensive diagnostic tests; treatment DSS, which support the decision between treatment modalities or treatment plans; and patient DSS, which focusses on informing the patient.

We described each study using the number of patients, the decision that the system supports, and the system inputs and outcomes. We also commented on the general applicability, the reported performance of the system and some of the primary limitations. In order to assess the reporting quality of the studies, we tested each paper for their compliance to the TRIPOD (Transparent Reporting of studies on prediction models for Individual Prognosis Or Diagnosis) reporting guideline ${ }^{[24]}$. We reported this in a percentage calculated using the document: "Scoring adherence of prediction model study reports to the TRIPOD reporting guideline", available on http://www.tripod-statement.org/.

\section{Results}

Included Studies

DSS for Diagnosis and Diagnostic Interventions

The studies that contained a diagnostic DSS are listed in Table 3.1. Two of the studies we found had as goal to support the use of a diagnostic tool: Lee et al. (2010) and Mukai et al. (2013). The application tested by Mukai et al. (2013) was meant to support the use of prostate specific antigen (PSA) tests for patients. The DSS was a web-based application that would aid in the decision to perform PSA tests for general practitioners (GPs) in Denmark. The study lead to the conclusion that it was possible to grant GPs in Denmark easy access to web-based DSS by replacing certain words in their medical records by hyperlinks. However it also showed that this DSS did not change PSA-testing behavior. Since this study neither developed nor validated this DSS, the compliance to the TRIPOD guidelines could not be tested.

Lee et al. (2010) attempted to support the use of a biopsy by predicting initial biopsy outcomes through three different models: support vector machine (SVM), artificial neural network (ANN) and multiple logistic regression. The study trained each of the models on 600 patients who had undergone transrectal ultrasonography (TRUS)-guided prostate biopsies, and tested them on 477 and compared the model performances. The parameters of the models were TRUS findings and clinical parameters, including age and PSA. The area under the receiver operating characteristic $(R O C)$ curve $(A \cup C)$ for the use of multiple logistic regression analysis, ANN, and the SVM were 0.768, 0.778, and 0.847, respectively, and pairwise comparison of the ROC curves showed that the SVM model had superior performance.

Kim et al. (2011), Sadoughi et al. (2014), Shah et al. (2012), van Leeuwen et al. 
(2017) and Chang et al. (1999) all aimed to detect, diagnose or classify PCa using a variety of methods. Kim et al. (2011) performed a study similar to Lee et al. (2010), but with as aim to improve pathological staging, rather than reduce the number of biopsies. Two models were developed, SVM and ANN, for the prediction of advanced PCa and compared these based on performance. The models used TRUS-guided biopsy parameters and were tested on 532 patients divided into training and test groups. The SVM model performed significantly better $(p=0.02)$ than the ANN model based on ROC curve, with an AUC 0.805 while that of the ANN model was 0.719. This study showed that these models could improve objective pathological staging of biopsy-proven PCa patients, and could be applied in combination with TRUS-guided biopsies once externally validated.

Another neural network was trained on laboratory results by Sadoughi et al. (2014) who then performed particle swarm optimization. The specific goal of this study was to aid in distinguishing between localized PCa and benign hyperplasia of the prostate. The model was internally validated on 60 patients, and the authors found an accuracy of $98.33 \%$. The description of the methodology was limited, and the reporting conformed only $33 \%$ to the TRIPOD guidelines. The model could potentially improve detection of PCa and possibly reduce the number of biopsies, but external validation is necessary.

Notable is Shah et al. (2012), who also used SVM, but in contrast to Lee et al. (2011), the model was not trained on biopsy results, but on pathological regions of a magnetic resonance imaging (MRI) scan of post prostatectomy prostates. The aim was not only to diagnose PCa, but to locate it specifically on MRI scans by modeling voxel specific risk analyses. The sensitivity and the specificity of the model with optimized SVM parameters was 90\%, and the Kappa coefficient was 80\%, where the raters were the DSS and the ground truth histology. The study only included 24 patients, but since the model was trained on specific regions, the training was done on 225 cancer and 264 non-cancerous regions. This model could be applied in any hospital with a 3.0 T endorectal MP-MRI scanner, although it still requires validation

Van Leeuwen et al. (2017) developed a nomogram, rather than a deep learning algorithm, that included a larger number of parameters to diagnose significant PCa. The nomogram included Prostate Imaging Reporting and Data System (PIRADS), age, PSA, digital rectal examination (DRE), prostate volume, and prior biopsy. The model performed with an AUC of 0.864 on an external validation set, and the paper proposed an optimal strategy to reduce the number of biopsies needed with minimal risk of under-diagnosis. This paper conformed to the TRIPOD guidelines for $97 \%$.

Chang et al. (1999) evaluated the usefulness and the performance of a DSS, the Prostate Cancer Expert System (PCES), which was validated on 43 patients with confirmed PCa. The PCES system, which utilized PSA, Gleason score, TRUS, and DRE was used to categorize the patients into localized or advanced PCa, and the same 
test was applied to four attending physicians and four residents. The results showed that the PCES performed with a higher accuracy than all residents and physicians, though the difference was only higher for two physicians. It also showed that after consultation of the PCES, the staging accuracy of the residents improved to the level of the attending physicians.

A number of prediction tools are currently being applied in the clinic to aid in the further diagnosis of the PCa disease, providing predictions on lymph node (LN) involvement, organ confinement (OC), seminal vesical (SV) involvement and extracapsular extension and risk of failure after treatment. The Partin tables ${ }^{[25,26]}$ are a set of nomograms to predict OC of PCa, initially introduced in 1993, and most recently updated in Tosoian et al. (2017) $)^{[27]}$. Based on these nomograms, Roach derived a set of formulas for the prediction of SV involvement in a paper published by Diaz et al. (1994) ${ }^{[28]}$. Roach et al. (1994) ${ }^{[29]}$ also derived formulas that predicted LN involvement based on PSA and Gleason score, and Roach et al. (2000) ${ }^{[30]}$ did the same for the risk of failure following radiotherapy (RT) and extracapsular extension in patients with localized PCa.

Tosoian et al. (2017) validated and updated the Partin tables on a patient cohort of 4459 patients with as goal to predict the pathological outcome after radical prostatectomy. The performance of the model was tested for binary regression where the AUC was calculated when comparing organ confined (OC) PCa to other pathological outcomes. The model performed best when predicting OC versus LN involvement (AUC $=0.918$ ) and versus seminal vesical $(S V)$ involvement (AUC = 0.856 ). The weakest performance was for OC versus extra-prostatic extension (AUC $=0.673)$.

Diaz et al. (1994) split patients into high risk and low risk groups of SV involvement using PSA and Gleason score, and tested this on 217 patients. The incidence rate of SV involvement in the low risk group was $7 \%$, while the incidence rate in the high risk group was 37\%, resulting in a chi-square of 23.17 .

Roach et al. (1994) performed a similar study on 282 patients, but divided the patients into low and high risk groups for $L N$ involvement. This resulted in $6 \%$ incidence rate in the low risk group and a $40 \%$ incidence rate in the high risk group, resulting in a significant split $(p<0.001)$.

Roach et al. (2000) split 895 patients into low, intermediate and high risk groups for extracapsular extension. This resulted in an incident rate of $17.8 \%, 46.7 \%$ and $66.7 \%$ incidence rate in low, intermediate and high risk groups respectively, which was a significant split $(p<0.01)$.

D’Amico et al. (1998) suggested a widely accepted risk classification for prostate cancer to help predict biochemical outcome after five years after PCa treatment, stratifying them into low, intermediate and high risk PCa ${ }^{[31]}$. This study included 1872 patients who underwent radical prostatectomy, EBRT or interstitial RT. Cox regression 
was used to calculate the relative risk between different groups of patients, based on risk level and treatment type. No validation was performed in this paper.

Memorial Sloan Kettering Cancer Centre (MSKCC) also has a set of nomograms publically available that is based on data from more than 10,000 patients. They have nomograms available to predict outcome after radical prostatectomy both pre- and post-treatment as well as after PSA elevation (see https://www.mskcc.org/ nomograms). 
Table 3.1 - Overview Diagnosis support systems

\begin{tabular}{|c|c|c|c|c|c|}
\hline Study & $N$ & Decision/Diagnose & Inputs & Outcomes & TRIPOD \\
\hline Roach $1994^{[28]}$ & 282 & $\begin{array}{l}\text { Low or high risk of LN } \\
\text { involvement }\end{array}$ & $\begin{array}{l}\text { PSA,GS, Clinical } \\
\text { stage }\end{array}$ & $\begin{array}{l}\text { P of LN } \\
\text { involvement }\end{array}$ & $79 \%$ \\
\hline Diaz $1994^{[27]}$ & 217 & $\begin{array}{l}\text { Low or high risk of SV } \\
\text { involvement }\end{array}$ & PSA, GS & $\begin{array}{l}\text { P of SV } \\
\text { involvement }\end{array}$ & $69 \%$ \\
\hline D’Amico $1998^{[30]}$ & 1872 & Patient risk group & $\begin{array}{l}\text { PSA, GS, } \\
\text { Clinical stage }\end{array}$ & $\begin{array}{l}\text { 5-year PSA } \\
\text { outcome }\end{array}$ & $72 \%$ \\
\hline Chang $1999^{[31]}$ & 43 & $\begin{array}{l}\text { Localized vs Advanced } \\
\text { PCa }\end{array}$ & $\begin{array}{l}\text { PSA, GS, TRUC, } \\
\text { DRE }\end{array}$ & $\begin{array}{l}\text { P advanced PCa } \\
\text { and P localized } \\
\text { PCa }\end{array}$ & $65 \%$ \\
\hline Roach $2000^{[29]}$ & 895 & Extracapsular extension & PSA, GS & $\begin{array}{l}\text { Extracapsular } \\
\text { extension risk }\end{array}$ & $92 \% * *$ \\
\hline Lee $2010^{[32]}$ & 1077 & Biopsy & $\begin{array}{l}\text { Clinical*, } \\
\text { Imaging*, PSA }\end{array}$ & P PCa & $86 \%$ \\
\hline $\operatorname{Kim} 2011^{[33]}$ & 532 & Advanced PCa & $\begin{array}{l}\text { TRUS, Clinical, } \\
\text { PSA }\end{array}$ & $\mathrm{P}$ advanced $\mathrm{PCa}$ & $79 \%$ \\
\hline Shah $2012^{[34]}$ & 31 & Location PCa & MRI image & $\begin{array}{l}\text { Cancer } \\
\text { probability map }\end{array}$ & $83 \%$ \\
\hline Mukai 2013 & 30773 & PSA test & Clinical & recommendation & $-* * *$ \\
\hline Sadoughi $2014^{[36]}$ & 360 & $\mathrm{PCa}$ & PSA, Age & P PCa & $33 \%$ \\
\hline $\begin{array}{l}\text { van Leeuwen } \\
2017^{[37]}\end{array}$ & 591 & Significant PCa & $\begin{array}{l}\text { Clinical, PSA, } \\
\text { PIRADS }\end{array}$ & $\mathrm{P}$ significant $\mathrm{PCa}$ & $97 \%$ \\
\hline Tosoian, $2017^{[26]}$ & 4459 & Pathological Stage & $\begin{array}{l}\text { PSA, GS, } \\
\text { Clinical stage }\end{array}$ & $\begin{array}{l}\% \text { likelihood of } \\
\text { given stage }\end{array}$ & $83 \%$ \\
\hline
\end{tabular}

Abbreviations. N: number of patients; P: probability; PCa: prostate cancer; PSA: prostate specific antigen; PIRADS: Prostate Imaging Reporting and Data System; TRUS: transrectal ultrasound scan; LN: Lymph Node; GS: Gleason Score; SV: seminal vesicles; TRIPOD: adherence to the TRIPOD statement; DRE: digital rectal examination

*Clinical, imaging and tumor parameters

**Abstract only

***No development or validation of DSS, so no TRIPOD evaluation possible

\section{DSS Supporting Treatment Decisions}

The studies that contained treatment DSS are listed in Table 3.2. The studies described by Walsh et al. (2018), Smith et al. (2016) and van Wijk et al. (2018) compare different RT treatment plans performed on the same patient and the DSS selects the best plan with most favorable outcome. This type of personalized DSS is very suitable for the comparison of RT modalities, as the treatment plans are a predictor for the delivered dose, with consequent treatment outcome. Walsh et al. (2018) used a combination of existing models for tumor control probability (TCP) and normal tissue complication probability (NTCP) to compare different treatment plans with photon and proton RT for 25 patients. The study included extensive 
corrections for displacements during treatment, which aided in the prediction of delivered dose. As this was a modelling study, no validation was done. However, the concept could be used as a basis for RT plan selection between different modalities and could aid in the optimization of TCP and NTCP. Smith et al. (2016) utilized an advanced Bayesian network to optimize intensity modulated radiotherapy (IMRT) treatment plans based on outcome in terms of progression free survival and toxicity. The models were validated against independent clinical trials for the metastasis free survival and overall survival, and resulted in uncertainties of $2.5 \%$ and $2 \%$ respectively. This method could potentially be implemented into any IMRT planning system and has the potential to improve the quality of treatment plans, resulting in optimized outcomes.

A device called the implantable rectum spacer (IRS) has been developed to spare the rectum during IMRT by increasing the distance between the anterior rectum wall and the prostate ${ }^{[39,40]}$. Van Wijk et al. (2018) made use of image deformation based on a virtual IRS ${ }^{[41,42]}$ : she published models to predict the sparing effect of an IRS before implanting the IRS. The model was tested in a proof of concept study with 16 patients, comparing the DSS outcome for the virtual IRS to the real IRS in the patients, and the median discrepancies in outcome was $1.8 \%$. Once validation has taken place, this DSS could be applied into any RT planning system, and has the potential to personalize treatment choice.

Two studies were found that supported the decisions involving follow-up treatment. (Reed et al. 2014) analyzed the cost-effectiveness (CE) of the use of a nucleic acid detection immunoassay (NADiA) ProsVueTM to support the decision for adjuvant radiotherapy (ART). This model showed that primarily for the intermediate risk patients, NADiA ProsVue had an incremental cost-effectiveness ratio (ICER) lower than $\$ 50,000$,- in $83.6 \%$ of the simulations. This analysis in its current state is not applicable outside the USA. Salem et al. (2018) validated a computer-led decision support system aimed to give additional advice in follow-up treatment strategy. This system uses the clinical profile of the patient in combination with blood test results to propose a follow-up strategy. Validation was done on 200 patients by comparing the computer generated strategy to the advice given by trained urologists. In all cases, the agreement between the experts and the system was better than moderate (kappa >0.6). The paper argued that such a system could significantly reduce costs in follow-up care in the United Kingdom, and believed the system can be used by any healthcare worker, regardless of urology background.

Hodges et al. (2012) developed a CE model to analyze the benefit of IMRT with respect to stereotactic body radiotherapy (SBRT). Though this study compared these two treatments, this study analyzed on cohort level and not case-specific, as is expected of personalized medicine. The CE analysis was done based solely based on probabilistic simulations, thus not taking into account variations in cost, transition rates, or utility 
values. A sensitivity analysis was performed, revealing that in $66 \%$ of the iterations SBRT was cost-effective over IMRT.

Vanneste et al. (2015) constructed a CE model to compare IMRT + IRS versus IMRT without IRS. He developed a decision-analytic Markov model to examine the effect of late rectal toxicity and compare the costs and quality-adjusted life years (QALYS). An ICER of $€ 55,880$ per QALY was gained. For a ceiling ratio of $€ 80,000$, IMRT+S had a $77 \%$ probability of being cost-effective.

Table 3.2 - Overview of Treatment support systems

\begin{tabular}{|c|c|c|c|c|c|}
\hline Study & $N$ & Decision & Inputs & Outcomes & TRIPOD \\
\hline $\begin{array}{l}\text { Hodges } \\
2012^{[43]}\end{array}$ & Model & SBRT, IMRT & $\begin{array}{l}\text { Utility, } \\
\text { transitions }\end{array}$ & QALY, Costs, ICER & $80 \%$ \\
\hline Reed $2014^{[44]}$ & Model & ART & Risk group & QALY, Cost, ICER & $80 \%$ \\
\hline $\begin{array}{l}\text { Vanneste } \\
2015^{[45]}\end{array}$ & Model & $\begin{array}{l}\text { IMRT+IRS, } \\
\text { IMRT }\end{array}$ & $\begin{array}{l}\text { ProsVue } \\
\text { slope }\end{array}$ & QALY, Cost, ICER & $81 \%$ \\
\hline Smith 2016 & Model & RT plan & $\begin{array}{l}\text { Utility, } \\
\text { transitions }\end{array}$ & TCP, NTCP, QALY & $87 \%$ \\
\hline $\begin{array}{l}\text { van Wijk } \\
2018^{[47]}\end{array}$ & 23 & IRS in RT & $\begin{array}{l}\text { DVH, } \\
\text { Clinical* }\end{array}$ & TCP, NTCP & $84 \%$ \\
\hline $\begin{array}{l}\text { Salem } \\
2018^{[48]}\end{array}$ & 200 & Follow-up & DVH, Clinical & $\begin{array}{l}\text { Follow-up } \\
\text { suggestion }\end{array}$ & $71 \%$ \\
\hline $\begin{array}{l}\text { Walsh } \\
2018^{[49]}\end{array}$ & 25 & $\begin{array}{l}\text { IMRT, V-mat, } \\
\text { PSPT, IMPT }\end{array}$ & $\begin{array}{l}\text { Symptoms, } \\
\text { Blood tests }\end{array}$ & $\begin{array}{l}\text { TCP, NTCP, } \\
\text { Robustness, stability }\end{array}$ & $84 \%$ \\
\hline
\end{tabular}

Abbreviations. N: number of patients; IMRT: intensity modulated radiotherapy; V-mat: volumetricmodulated arc therapy; PSPT: passively scattered protontherapy; IMPT: image modulated proton therapy; TCP: tumor control probability; NTCP: normal tissue complication probability; DVH: dose-volumehistogram; QALY: quality adjusted life year; IRS: implantable rectum spacer; ART: adjuvant radiotherapy; ICER: incremental cost-effectiveness ratio; TRIPOD:adherence to the TRIPOD statement.

*Clinical parameters.

\section{DSS Focusing on Providing Patient Information}

This section describes the studies that attempted to improve shared decisionmaking. This type of DSS fosters patient involvement in therapeutic decisions, and emphasizes the provision of information needed to make such decisions (Table 3.3). Berry et al. (2013) described the testing of a personal patient-profile (P3P) intervention for PCa patients. P3P is a web-based program to help men prepare for shared decision making about the management of early stage prostate cancer. The study hypothesized that when P3P was used in combination with a consultation with different clinicians to prepare for decision making, patients were more satisfied with their treatment choice and experienced less regret, but testing did not result in significant improvement. However, this type of system has the capability to take into account patient preferences 
and priorities, while when only using consultations, these preferences can be misinterpreted by clinicians. The paper suggested similar studies to be performed in the future on larger cohorts.

Nguyen et al. (2009) performed an extensive literature research for predictive outcome models for 15 different treatment options for PCa, including active surveillance, combinations of radical prostatectomy, RT, hormonal therapy and high intensity focused ultrasound. They attempted to create a comprehensive overview of the different outcome combinations, such as survival, metastasis, and various toxicities. This overview was designed to be comprehensive for patients so that they could use their own priorities and preferences to make an informed decision. Though they concluded that there is a great need for additional models, this paper provided a framework and is a step towards evidence-based personalized medicine. Additionally, this framework could be a useful tool for clinical decision making by medical personnel when adapted for these users.

Table 3.3 - Summary of patient support systems

\begin{tabular}{l|lllll}
\multicolumn{1}{l}{ Study } & N & Decision & Inputs & Outcomes & TRIPOD \\
\hline Nguyen 2009[50] & Literature & Treatment & Various & Various & $86 \%$ \\
Berry 2013 & $494]$ & Treatment & P3P & Decision satisfaction & $82 \%$ \\
\hline
\end{tabular}

Abbreviations. N: number of patients; P3P: personal patient-profile for prostate cancer.

\section{Excluded studies}

Two studies, Stacey et al. (2016) $)^{[52]}$ and Jayadevappa et al. (2015) ${ }^{[53]}$ were excluded because they contained study proposals. Both these studies will attempt to test patient decision aids. Another study, McRae et al. (2016) ${ }^{[54]}$ was excluded because it was not about prostate cancer.

Eight studies in total were excluded as they did not describe a DSS, but instead provided tools that could be used in the development of a DSS. Though these studies can be used to further personalize PCa treatment, these tools cannot be applied in the clinic in their current form. A short overview of these papers is given in Table 3.4.

Two studies, Daemen et al. (2009) and Beyan et al. (2014) attempted to use genetic information to improve cancer prediction outcomes. Beyan specifically worked to incorporate single nucleotide polymorphisms (SNPs) into the national health information system of Turkey.

Zumsteg et al. (2013) ${ }^{[55]}$, Gnanapragasam et al. (2016) ${ }^{[56]}$ and Epstein et al. (2016) [57] provided new definitions for risk group stratification, increasing upon the current low, intermediate and high risk groups. They found clear separation in progression free survival in their new risk groups, making these a potential tool for diagnostic DSS. Gries et al. (2017) ${ }^{[58]}$ provided a method to interpolate the utility values for all combinations of 18 different health states, which could be a valuable tool for DSS to quantify QALYS. 
Kuru et al. (2013) ${ }^{[59]}$ showed that a PIRADS score on multiparametric magnetic resonance imaging (mpMRI) is prognostic for PCa.

Finally Kent et al. (2015) ${ }^{[60]}$ performed a literature search and attempted to create a diagnostic tool which would predict the life expectancy in PCa patients. They concluded that no existing model was suitable for incorporation into a DSS.

Table 3.4 - Overview of excluded studies that described tools to improve DSS

\begin{tabular}{|c|c|c|c|c|}
\hline Study & $N$ & Tool & Inputs & Outcomes \\
\hline Daemen 2009[61] & 55 & Genetic integration & DNA, CNV & Cancer outcome \\
\hline Kuru 2013[59] & 50 & Diagnostics & mpMRI & PIRADS \\
\hline Zumsteg 2013 $3^{[55]}$ & 1024 & Risk stratification & $\begin{array}{l}\text { Risk factors, Gleason } \\
\text { score, biopsy }\end{array}$ & Risk group \\
\hline Beyan $2014^{[62]}$ & Model & Genetic integration & SNPS & Various \\
\hline Kent $2015^{[60]}$ & Model & Diagnostics & $\begin{array}{l}\text { Clinical and tumor } \\
\text { features }\end{array}$ & Life expectancy \\
\hline $\begin{array}{l}\text { Gnanapragasam } \\
2016^{[56]}\end{array}$ & 10139 & Risk stratification & $\begin{array}{l}\text { PSA, stage, Gleason } \\
\text { score }\end{array}$ & Risk group \\
\hline Epstein 2016 $[57]$ & 26325 & Risk stratification & PSA, stage, & Gleason grade \\
\hline Gries $2017^{[58]}$ & 120 & Utility values & $\begin{array}{l}\text { Utility's } 18 \text { health } \\
\text { states }\end{array}$ & $\begin{array}{l}\text { Utility's } 243 \\
\text { heath states }\end{array}$ \\
\hline
\end{tabular}

Abbreviations. N: Number of patients; CNV: copy number variation; SNP: single nucleotide polymorphism; PSA: prostate specific antigen; $m p M R I$ :multiparametric magnetic resonance imaging; PIRADS: Prostate Imaging Reporting and Data system.

\section{Discussion}

\section{Primary findings}

In response to the increasing number of PCa treatment options, the development of DSS has become a growing topic of interest to provide an aid in difficult medical decisions. Diagnosis and staging DSS have also been a growing topic of interest, as well as a number of tools to improve cancer detection, predictions of treatment outcome and outcome stratification. However, there is still a need for new DSS in treatment decision aids and validation of diagnostic DSS. Additionally, the field of patient informed decision-making is still in its infancy, but essential for the growth towards individualized medicine.

For the diagnostic tools, there are a number of viable tools available for the diagnosis of local PCa that have been validated on large cohorts ${ }^{[33,34,36,38,63]}$. Additionally, a study has been performed for detection of PCa on the voxel level for MRI images ${ }^{[35]}$. This type of diagnostic DSS could additionally assist in treatment planning or treatment selection. One study explores diagnosis of PCa with LN metastasis ${ }^{[64]}$, but this field remains largely uncharted, similar to the computer automated detection of biochemical failure or treatment failure, also explored in only one study ${ }^{[48]}$. 
For treatment DSS, the proportion between the number of treatment options and the decision support tools remains somewhat skewed. Nguyen et al. (2009) ${ }^{[50]}$ suggested 15 different combinations of treatment options, and this did not include the use of rectal displacement devices ${ }^{[65,66]}$ or proton therapy. The treatment DSS that were found by the current study primarily involved RT, including treatment plan selection ${ }^{[46]}$, proton compared to photon therapy ${ }^{[49]}$, SBRT compared to $I M R T^{[43]}$, and the use of an implantable rectum spacer in EBRT ${ }^{[47]}$. Nguyen et al. (2009) attempted to create a general overview of prediction tools to create a clear overview for patients, and found most prediction tools to be focused on RT and radical prostatectomy. Less prediction tools are available for brachytherapy, which is a very viable treatment option for PCa. One study is Alitto et al. (2017) ${ }^{[67]}$ describes an Umbrella Protocol for the standardized development of new DSS. This protocol could help in improving the application of new DSS in clinics.

Development of patient decision aids is challenging, as cultural and language barriers are much more present in this field of research. Nguyen et al. (2009) developed a comprehensive treatment overview for patients, but found that the predictive tools available were limited, leaving an incomplete overview. Berry et al. (2013) ${ }^{[51]}$ hypothesized that patient satisfaction was increased after treatment when they were actively involved in the decision-making process, as well as comorbidities like anxiety, depression and fatigue was reduced, and proceeded to test this using the P3P intervention. He has found, however, that this method has not increased self-reported preparation for the intervention.

\section{DSS Design}

The last decade deep learning algorithms have gained popularity in the development of DSS for the classification of cancers, and the same is true within the field of PCa. Kim et al. (2011) and Lee et al. (2010) tackled a similar problem with both an ANN and an SVM, but the dataset of Kim et al. (2011) was approximately double in size. This increased patient cohort resulted in a higher AUC performance for both models, which confirms that deep learning algorithms rely heavily on large datasets. Also, no external validation was done, so currently these models are not generally applicable. An approach to make these models both more accurate and applicable for clinical use would be to use distributed learning, where the models are trained on centers all over the world, without the data having to leave the clinics. Shah et al. (2012) used an SVM for a more complex problem than just classification by doing voxel based analyses for the localization of PCa. The drawback of this model is that for training, a large amount of imaging data must be available, as well as 3D pathology, but the advantage is that the usage of sub-regions in the prostate allows for data augmentation.

Notable is that a number of nomograms initially developed more than two 
decades ago are currently still in use, though updated using newer datasets. The continued application of simple, easy to interpret models is something to keep in mind in the development of new DSS. Though artificial intelligence has the potential to improve diagnosis of PCa, transparency plays a large role in clinical application. It also shows that predictive parameters for PCa are very consistent, with persistent usage of Gleason score, PSA, Clinical stage and age. Any new DSS being developed should be tested against the performance of these parameters to avoid tackling a simple problem using computationally heavy machine learning.

When looking at the available treatment DSS, we see that most of these are focused around RT. This is likely due to the patient specific treatment planning done in RT, which result in highly detailed dose maps before the start of treatment. This is ideal for DSS, as different treatment plans can be directly compared, and the different outcomes can be predicted using dose response curves. Other types of treatment, such as prostatectomy or watchful waiting rely much more heavily on clinical parameters and tumor parameters for predictions of their outcome or on subjective physician decisions. This makes it harder to compare outcomes of different treatments for the same patient. The development of new DSS comparing completely different treatment types with each other for the same patient would be very beneficial for filling this gap in the current literature.

\section{Study Limitations}

This study focused on DSS in PCa, and the scope did not include any other cancer types. It is possible that general DSS, applicable for more than one cancer type were therefore overlooked. The search was also focused on DSS, and thus did not include any predictive models that could aid in decision-making. The terms describing DSS may not have been used in interesting studies that could have been included in this overview. Patient decision aids was also not a search term, which may be the cause of the limited number of DSS for patients included in this overview. The search performed was only MEDLINE/PubMed linked, so studies not available on this medium were overlooked.

Inherent to literature overviews is that negative findings are not always reported, so failed attempts at creating DSS are often uncommon in overviews and literature reviews, and this may cause a biased view on the subject.

We assessed the reporting quality of each included study using the TRIPOD statement for quantification and comparison. Although the TRIPOD statement has been endorsed by a large number of medical journals and editorial organizations, it is not an universal gold standard. The checklist used for the assessment has limitations, such as the severe point punishment for the lack of specific keywords in title or abstract. This could reduce the score of a well-written paper. Additionally, the adherence to the TRIPOD statement was compressed 
into a single number, and the specific reporting issues were not named, such as improper analyses, lack of validation or reporting of study specifics. However, it is a useful tool to show the strength of a report, as when adherence to the TRIPOD statement was high, the paper was clearly written, and all proper steps were taken for the development of quality DSS tools.

\section{Conclusion}

A number of DSS for the primary diagnosis and staging of localized PCa are available. Treatment DSS were mostly focused on EBRT, for which several tools are available. However, a lack of DSS for other treatment modalities suggests that the development of new tools are necessary to compare objectively different treatment modalities. The development of patient decision aids is a new field of research, and few successes have been made for PCa patients. Though the idea of informed decision making by patients is in line with the goal of personalized medicine, the development of these tools needs to overcome a number of barriers to be successful, like comprehensiveness, language barriers, patient cooperation, physician cooperation, etc. More research needs to be performed to better empower clinical decisions in the diagnosis and treatment process.

\section{Conflicts of Interest}

Dr. Philippe Lambin reports grants/sponsored research agreements from Varian medical, Oncoradiomics, ptTheragnostic and outside the submitted work, from Health Innovation Ventures and DualTpharma. He received an advisor (SAB)/ presenter fee and/or reimbursement of travel costs/external grant writing fee and/ or in kind manpower contribution from Oncoradiomics and, outside the submitted work, BHV and Convert pharmaceuticals. Dr Lambin has shares in the company Oncoradiomics SA and, outside the submitted work, Convert pharmaceuticals SA and is co-inventor of two patents on radiomics (PCT/NL2014/050248, PCT/ NL2014/050728) licensed to Oncoradiomics and one patent on mtDNA (PCT/ EP2014/059089) licensed to ptTheragnostic/DNAmito, three non-patentable invention (softwares) licensed to ptTheragnostic/DNAmito, Oncoradiomics and Health Innovation Ventures.Dr. Seán Walsh is a paid employee at Oncoradiomics. Yvonka van wijk is an employee at ptTheragnostics. Iva Halilaj is a paid employee at Health Innovations. 


\section{References}

1. Bray, F., J. Ferlay, I. Soerjomataram, et al., Global cancer statistics 2018: GLOBOCAN estimates of incidence and mortality worldwide for 36 cancers in 185 countries. CA Cancer J Clin, 2018. 68(6): p. 394-424.

2. Hamdy, F.C., J.L. Donovan, J.A. Lane, et al., 10-Year Outcomes after Monitoring, Surgery, or Radiotherapy for Localized Prostate Cancer. N Engl J Med, 2016. 375(15): p. 1415-1424.

3. Lennernas, B., K. Majumder, J.E. Damber, et al., Radical prostatectomy versus high-dose irradiation in localized/locally advanced prostate cancer: A Swedish multicenter randomized trial with patient-reported outcomes. Acta Oncol, 2015. 54(6): p. 875-81.

4. Donovan, J.L., F.C. Hamdy, J.A. Lane, et al., Patient-Reported Outcomes after Monitoring, Surgery, or Radiotherapy for Prostate Cancer. N Engl J Med, 2016. 375(15): p. 1425-1437.

5. Stone, N.N., L. Potters, B.J. Davis, et al., Multicenter analysis of effect of high biologic effective dose on biochemical failure and survival outcomes in patients with Gleason score 7-10 prostate cancer treated with permanent prostate brachytherapy. Int J Radiat Oncol Biol Phys, 2009. 73(2): p. 341-6.

6. Sylvester, J.E., P.D. Grimm, J.C. Blasko, et al., 15-Year biochemical relapse free survival in clinical Stage T1-T3 prostate cancer following combined external beam radiotherapy and brachytherapy; Seattle experience. Int J Radiat Oncol Biol Phys, 2007. 67(1): p. 57-64.

7. Ragde, H., G.L. Grado, and B.S. Nadir, Brachytherapy for clinically localized prostate cancer: thirteen-year disease-free survival of 769 consecutive prostate cancer patients treated with permanent implants alone. Arch Esp Urol, 2001. 54(7): p. 739-47.

8. Grimm, P., I. Billiet, D. Bostwick, et al., Comparative analysis of prostate-specific antigen free survival outcomes for patients with low, intermediate and high risk prostate cancer treatment by radical therapy. Results from the Prostate Cancer Results Study Group. BJU Int, 2012. 109 Suppl 1: p. 22-9.

9. Musunuru, H.B., T. Yamamoto, L. Klotz, et al., Active Surveillance for Intermediate Risk Prostate Cancer: Survival Outcomes in the Sunnybrook Experience. J Urol, 2016. 196(6): p. 1651-1658.

10. Agarwal, G., D. Buethe, C. Russell, et al., Long term survival and predictors of disease reclassification in patients on an active surveillance protocol for prostate cancer. Can J Urol, 2016. 23(2): p. 82159.

11. Soloway, M. and M. Roach, 3rd, Prostate cancer progression after therapy of primary curative intent: a review of data from prostate-specific antigen era. Cancer, 2005. 104(11): p. 2310-22.

12. Roach, M., 3rd, Radical prostatectomy or external beam radiotherapy: one step forward or two steps back? Cancer, 2002. 95(2): p. 215-8.

13. Vanneste, B.G., E.J. Van Limbergen, E.N. van Lin, et al., Prostate Cancer Radiation Therapy: What Do Clinicians Have to Know? Biomed Res Int, 2016. 2016: p. 6829875.

14. Valentini, V., N. Dinapoli, and A. Damiani, The future of predictive models in radiation oncology: from extensive data mining to reliable modeling of the results. Future Oncol, 2013. 9(3): p. 311-3.

15. Dekker, A., S. Vinod, L. Holloway, et al., Rapid learning in practice: a lung cancer survival decision support system in routine patient care data. Radiother Oncol, 2014. 113(1): p. 47-53. 
16. Abernethy, A.P., L.M. Etheredge, P.A. Ganz, et al., Rapid-learning system for cancer care. J Clin Oncol, 2010. 28(27): p. 4268-74.

17. Lambin, P., R.G. van Stiphout, M.H. Starmans, et al., Predicting outcomes in radiation oncology-multifactorial decision support systems. Nat Rev Clin Oncol, 2013. 10(1): p. 27-40.

18. Lambin, P., J. Zindler, B. Vanneste, et al., Modern clinical research: How rapid learning health care and cohort multiple randomised clinical trials complement traditional evidence based medicine. Acta Oncol, 2015. 54(9): p. 1289-300.

19. Lambin, P., E. Roelofs, B. Reymen, et al., 'Rapid Learning health care in oncology' - an approach towards decision support systems enabling customised radiotherapy'. Radiother Oncol, 2013. 109(1): p. 159-64.

20. Lambin, P., S.F. Petit, H.J. Aerts, et al., The ESTRO Breur Lecture 2009. From population to voxel-based radiotherapy: exploiting intra-tumour and intra-organ heterogeneity for advanced treatment of non-small cell lung cancer. Radiother Oncol, 2010. 96(2): p. 145-52.

21. Lambin, P., J. Zindler, B.G. Vanneste, et al., Decision support systems for personalized and participative radiation oncology. Adv Drug Deliv Rev, 2017. 109: p. 131-153.

22. Pawloski, P.A., G.A. Brooks, M.E. Nielsen, et al., A Systematic Review of Clinical Decision Support Systems for Clinical Oncology Practice. J Natl Compr Canc Netw, 2019. 17(4): p. 331-338.

23. McNutt, T.R., S.H. Benedict, D.A. Low, et al., Using Big Data Analytics to Advance Precision Radiation Oncology. Int J Radiat Oncol Biol Phys, 2018. 101(2): p. 285-291.

24. Moons, K.G., D.G. Altman, J.B. Reitsma, et al., New Guideline for the Reporting of Studies Developing, Validating, or Updating a Multivariable Clinical Prediction Model: The TRIPOD Statement. Adv Anat Pathol, 2015. 22(5): p. 303-5.

25. Partin, A.W., J. Yoo, H.B. Carter, et al., The use of prostate specific antigen, clinical stage and Gleason score to predict pathological stage in men with localized prostate cancer. J Urol, 1993. 150(1): p. 110-4.

26. Partin, A.W., L.A. Mangold, D.M. Lamm, et al., Contemporary update of prostate cancer staging nomograms (Partin Tables) for the new millennium. Urology, 2001. 58(6): p. 843-8.

27. Tosoian, J.J., M. Chappidi, Z. Feng, et al., Prediction of pathological stage based on clinical stage, serum prostate-specific antigen, and biopsy Gleason score: Partin Tables in the contemporary era. BJU Int, 2017. 119(5): p. 676-683.

28. Diaz, A., M. Roach, 3rd, C. Marquez, et al., Indications for and the significance of seminal vesicle irradiation during 3D conformal radiotherapy for localized prostate cancer. Int J Radiat Oncol Biol Phys, 1994. 30(2): p. 323-9.

29. Roach, M., 3rd, C. Marquez, H.S. Yuo, et al., Predicting the risk of lymph node involvement using the pre-treatment prostate specific antigen and Gleason score in men with clinically localized prostate cancer. Int J Radiat Oncol Biol Phys, 1994. 28(1): p. 33-7.

30. Roach, M., 3rd, A. Chen, J. Song, et al., Pretreatment prostate-specific antigen and Gleason score predict the risk of extracapsular extension and the risk of failure following radiotherapy in patients with clinically localized prostate cancer. Semin Urol Oncol, 2000. 18(2): p. 108-14.

31. D’Amico, A.V., R. Whittington, S.B. Malkowicz, et al., Biochemical outcome after radical 
prostatectomy, external beam radiation therapy, or interstitial radiation therapy for clinically localized prostate cancer. JAMA, 1998. 280(11): p. 969-74.

32. Chang, P.L., Y.C. Li, T.M. Wang, et al., Evaluation of a decision-support system for preoperative staging of prostate cancer. Med Decis Making, 1999. 19(4): p. 419-27.

33. Lee, H.J., S.I. Hwang, S.M. Han, et al., Image-based clinical decision support for transrectal ultrasound in the diagnosis of prostate cancer: comparison of multiple logistic regression, artificial neural network, and support vector machine. Eur Radiol, 2010. 20(6): p. 1476-84.

34. Kim, S.Y., S.K. Moon, D.C. Jung, et al., Pre-operative prediction of advanced prostatic cancer using clinical decision support systems: accuracy comparison between support vector machine and artificial neural network. Korean J Radiol, 2011. 12(5): p. 588-94.

35. Shah, V., B. Turkbey, H. Mani, et al., Decision support system for localizing prostate cancer based on multiparametric magnetic resonance imaging. Med Phys, 2012. 39(7): p. 4093-103.

36. Mukai, T.O., F. Bro, F. Olesen, et al., To test or not: a registry-based observational study of an online decision support for prostate-specific antigen tests. Int J Med Inform, 2013. 82(10): p. 973-9.

37. Sadoughi, F. and M. Ghaderzadeh, A hybrid particle swarm and neural network approach for detection of prostate cancer from benign hyperplasia of prostate. Stud Health Technol Inform, 2014. 205: p. 481-5.

38. van Leeuwen, P.J., A. Hayen, J.E. Thompson, et al., A multiparametric magnetic resonance imaging-based risk model to determine the risk of significant prostate cancer prior to biopsy. BJU Int, 2017. 120(6): p. 774-781.

39. Vanneste, B.G.L., K. van De Beek, L. Lutgens, et al., Implantation of a biodegradable rectum balloon implant: tips, Tricks and Pitfalls. Int Braz J Urol, 2017. 43(6): p. 1033-1042.

40. Vanneste, B.G.L., F. Buettner, M. Pinkawa, et al., Ano-rectal wall dose-surface maps localize the dosimetric benefit of hydrogel rectum spacers in prostate cancer radiotherapy. Clin Transl Radiat Oncol, 2019. 14: p. 17-24.

41. van Wijk, Y., B.G.L. Vanneste, S. Walsh, et al., Development of a virtual spacer to support the decision for the placement of an implantable rectum spacer for prostate cancer radiotherapy: Comparison of dose, toxicity and cost-effectiveness. Radiother Oncol, 2017. 125(1): p. 107-112.

42. Vanneste, B.G.L., Y. van Wijk, L.C. Lutgens, et al., Dynamics of rectal balloon implant shrinkage in prostate VMAT : Influence on anorectal dose and late rectal complication risk. Strahlenther Onkol, 2018. 194(1): p. 31-40.

43. Hodges, J.C., Y. Lotan, T.P. Boike, et al., Cost-effectiveness analysis of SBRT versus IMRT: an emerging initial radiation treatment option for organ-confined prostate cancer. Am J Manag Care, 2012. 18(5): p. e186-93.

44. Reed, S.D., S.B. Stewart, C.D. Scales, Jr., et al., A framework to evaluate the cost-effectiveness of the NADiA ProsVue slope to guide adjuvant radiotherapy among men with high-risk characteristics following prostatectomy for prostate cancer. Value Health, 2014. 17(5): p. 545-54.

45. Vanneste, B.G., M. Pijls-Johannesma, L. Van De Voorde, et al., Spacers in radiotherapy treatment of prostate cancer: is reduction of toxicity cost-effective? Radiother Oncol, 2015. 114(2): p. 276-81. 
46. Smith, W.P., M. Kim, C. Holdsworth, et al., Personalized treatment planning with a model of radiation therapy outcomes for use in multiobjective optimization of IMRT plans for prostate cancer. Radiat Oncol, 2016. 11: p. 38.

47. van Wijk, Y., B.G.L. Vanneste, A. Jochems, et al., Development of an isotoxic decision support system integrating genetic markers of toxicity for the implantation of a rectum spacer. Acta Oncol, 2018: p. 1-7.

48. Salem, H.A., G. Caddeo, J. McFarlane, et al., A multicentre integration of a computer-led follow-up of prostate cancer is valid and safe. BJU Int, 2018.

49. Walsh, S., E. Roelofs, P. Kuess, et al., Towards a Clinical Decision Support System for External Beam Radiation Oncology Prostate Cancer Patients: Proton vs. Photon Radiotherapy? A Radiobiological Study of Robustness and Stability. Cancers (Basel), 2018. 10(2).

50. Nguyen, C.T. and M.W. Kattan, Development of a prostate cancer metagram: a solution to the dilemma of which prediction tool to use in patient counseling. Cancer, 2009. 115(13 Suppl): p. 3039-45.

51. Berry, D.L., Q. Wang, B. Halpenny, et al., Decision preparation, satisfaction and regret in a multicenter sample of men with newly diagnosed localized prostate cancer. Patient Educ Couns, 2012. 88(2): p. 262-7.

52. Stacey, D., M. Taljaard, J. Smylie, et al., Implementation of a patient decision aid for men with localized prostate cancer: evaluation of patient outcomes and practice variation. Implement Sci, 2016. 11(1): p. 87.

53. Jayadevappa, R., S. Chhatre, J.J. Gallo, et al., Treatment preference and patient centered prostate cancer care: Design and rationale. Contemp Clin Trials, 2015. 45(Pt B): p. 296-301.

54. McRae, M.P., G. Simmons, J. Wong, et al., Programmable Bio-nanochip Platform: A Point-of-Care Biosensor System with the Capacity To Learn. Acc Chem Res, 2016. 49(7): p. 1359-68.

55. Zumsteg, Z.S., D.E. Spratt, I. Pei, et al., A new risk classification system for therapeutic decision making with intermediate-risk prostate cancer patients undergoing dose-escalated externalbeam radiation therapy. Eur Urol, 2013. 64(6): p. 895-902.

56. Gnanapragasam, V.J., A. Lophatananon, K.A. Wright, et al., Improving Clinical Risk Stratification at Diagnosis in Primary Prostate Cancer: A Prognostic Modelling Study. PLoS Med, 2016. 13(8): p. e1002063.

57. Epstein, J.I., M.J. Zelefsky, D.D. Sjoberg, et al., A Contemporary Prostate Cancer Grading System: A Validated Alternative to the Gleason Score. Eur Urol, 2016. 69(3): p. 428-35.

58. Gries, K.S., D.A. Regier, S.D. Ramsey, et al., Utility Estimates of Disease-Specific Health States in Prostate Cancer from Three Different Perspectives. Appl Health Econ Health Policy, 2017. 15(3): p. 375-384.

59. Kuru, T.H., M.C. Roethke, P. Rieker, et al., Histology core-specific evaluation of the European Society of Urogenital Radiology (ESUR) standardised scoring system of multiparametric magnetic resonance imaging (mpMRI) of the prostate. BJU Int, 2013. 112(8): p. 1080-7.

60. Kent, M. and A.J. Vickers, A systematic literature review of life expectancy prediction tools for patients with localized prostate cancer. J Urol, 2015. 193(6): p. 1938-42. 
61. Daemen, A., O. Gevaert, F. Ojeda, et al., A kernel-based integration of genome-wide data for clinical decision support. Genome Med, 2009. 1(4): p. 39.

62. Beyan, T. and Y. Aydin Son, Incorporation of personal single nucleotide polymorphism (SNP) data into a national level electronic health record for disease risk assessment, part 1: an overview of requirements. JMIR Med Inform, 2014. 2(2): p. e15.

63. Ghaderzadeh, M., Clinical decision support system for early detection of prostate cancer from benign hyperplasia of prostate. Stud Health Technol Inform, 2013. 192: p. 928.

64. Chang, P.L., T.M. Wang, S.T. Huang, et al., Use of a medical decision support system to improve the preoperative diagnosis of prostate cancer with pelvic lymph node metastases. Changgeng Yi Xue Za Zhi, 1999. 22(4): p. 556-64.

65. Wilton, L., M. Richardson, S. Keats, et al., Rectal protection in prostate stereotactic radiotherapy: a retrospective exploratory analysis of two rectal displacement devices. J Med Radiat Sci, 2017. 64(4): p. 266-273.

66. Mok, G., E. Benz, J.P. Vallee, et al., Optimization of radiation therapy techniques for prostate cancer with prostate-rectum spacers: a systematic review. Int J Radiat Oncol Biol Phys, 2014. 90(2): p. 278-88.

67. Alitto, A.R., R. Gatta, B. Vanneste, et al., PRODIGE: PRediction models in prOstate cancer for personalized meDIcine challenGE. Future Oncol, 2017. 13(24): p. 2171-2181. 

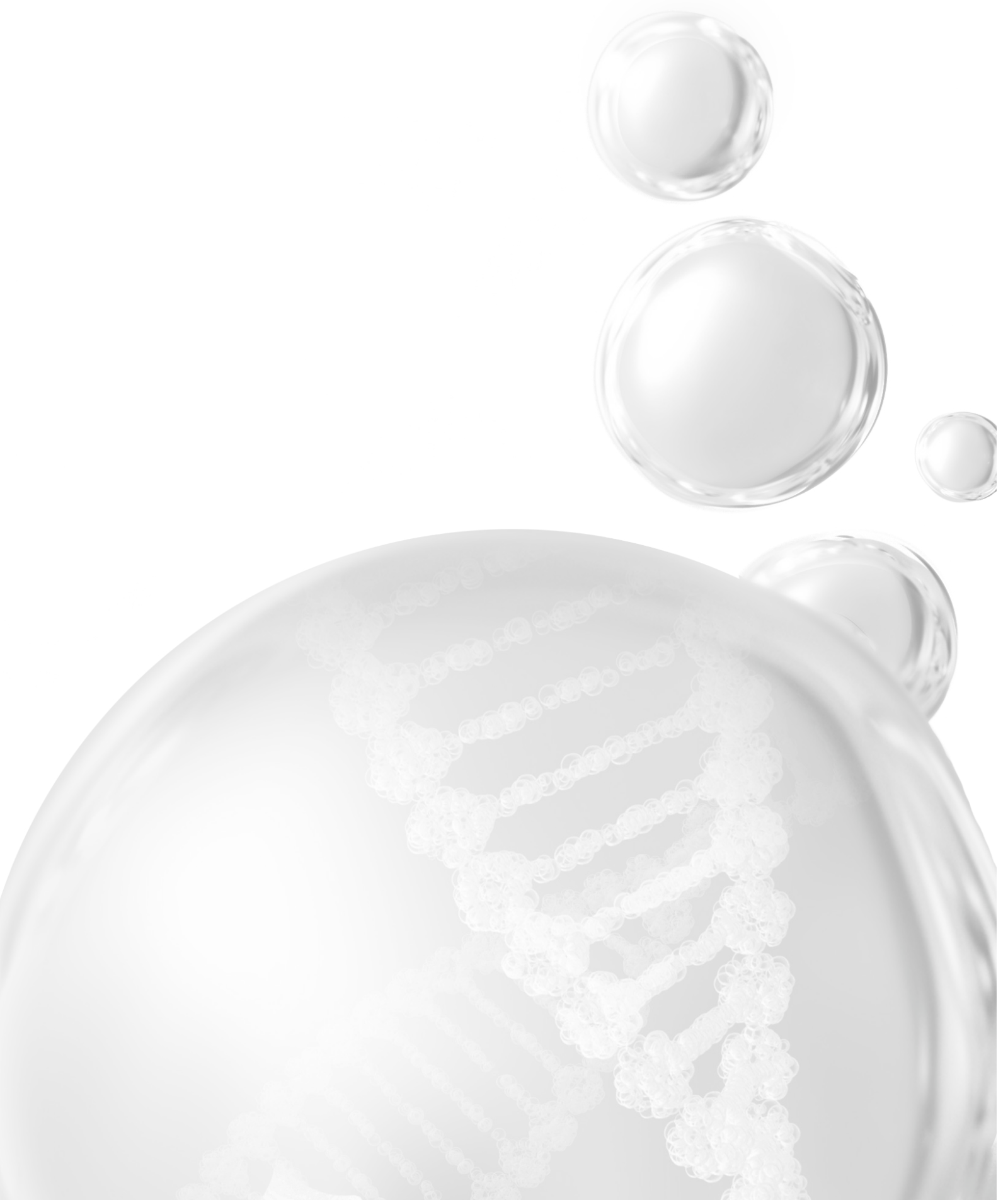
Treatment decision support systems for prostate cancer 

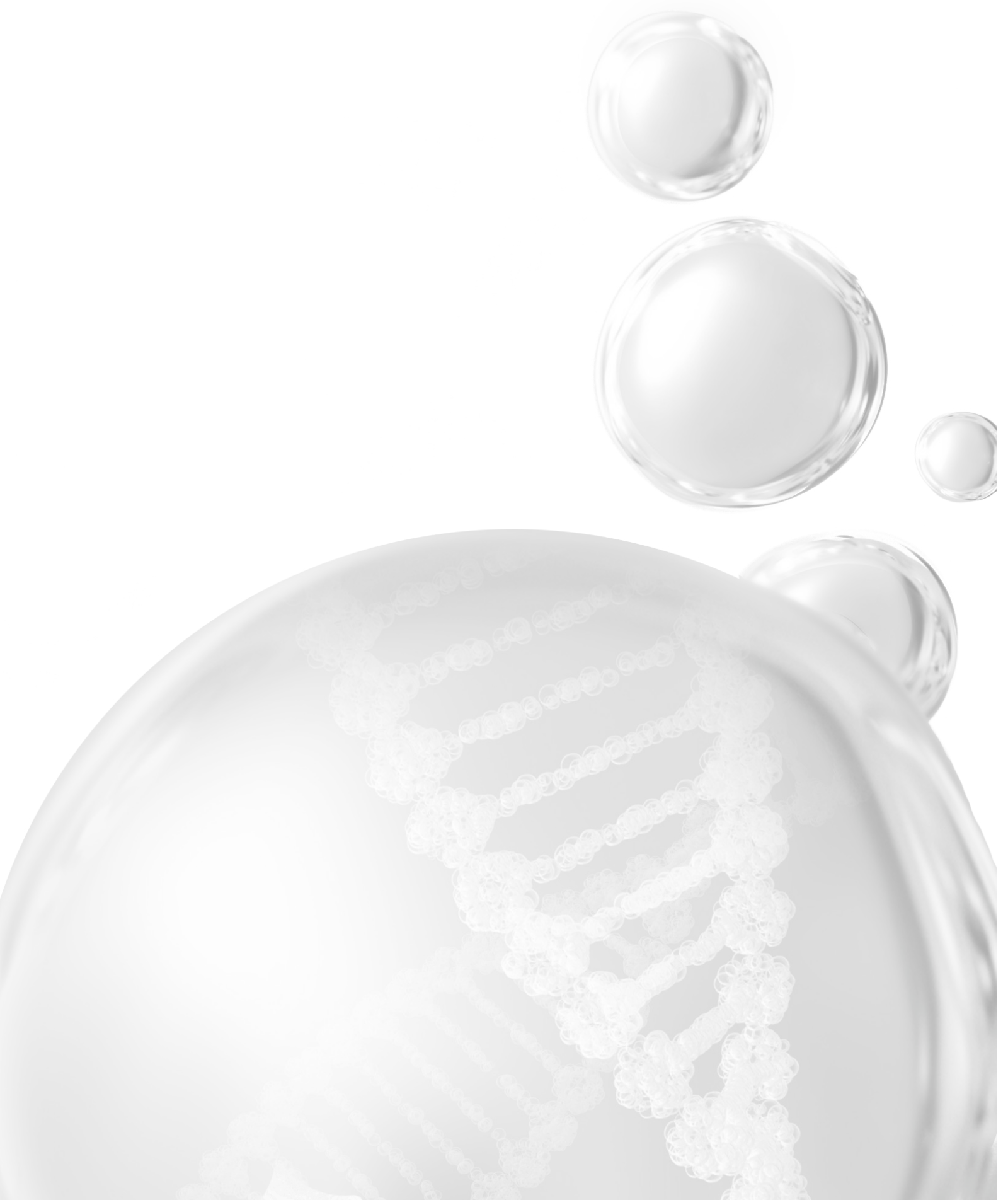


\section{Chapter 4}

\section{Development of a virtual implantable rectum spacer as the basis for a decision support system}

Authors:

Yvonka van Wijk, Ben Vanneste, Sean Walsh, Skadi van der Meer, Bram Ramaekers, Wouter van Elmpt, Michael Pinkawa, Philippe Lambin

Adapted from:

"Development of a virtual spacer to support the decision for the placement of an implantable rectum spacer for prostate cancer radiotherapy: Comparison of dose, toxicity and cost-effectiveness." Radiotherapy and Oncology 125.1 (2017): 107-112.

DOI: 10.1016/j.radonc.2017.07.026 


\begin{abstract}
Background: Previous studies have shown that the implantable rectum spacer (IRS) is not beneficial for all patients. A virtual IRS (V-IRS) was constructed to help identify the patients for whom it is cost-effective to implant an IRS, and its viability as a tool to tailor the decision of an IRS implantation to be beneficial for the specified patient was assessed. Please watch animation:
\end{abstract}

\title{
(https://www.youtube.com/watch?v=tDlagSXMKqw)
}

Methods: The V-IRS was tested on 16 patients: 8 with a rectal balloon implant (RBI) and 8 with a hydrogel spacer. A V-IRS was developed using 7 computed tomography (CT) scans of patients with a RBI. To examine the V-IRS, CT scans before and after the implantation of an IRS were used. IMRT plans were made based on CT scans before the IRS, after IRS and with the V-IRS, prescribing 70 Gray (Gy) to the planning target volume. Toxicity was accessed using externally validated normal tissue complication probability (NTCP) models, and the Cost-effectiveness was analyzed using a published Markov model.

Results: The rectum volume receiving 75 Gy (V75) were improved by both the IRS and the V-IRS with on average $4.2 \%$ and $4.3 \%$ respectively. The largest NTCP reduction resulting from the IRS and the V-IRS was $4.0 \%$ and $3.9 \%$ respectively. The RBI was cost-effective for 1 out of 8 patient, and the hydrogel was effective for 2 out of 8 patients, and close to effective for a third patient. The classification accuracy of the model, regarding cost-effectiveness, was $100 \%$.

Conclusions: The V-IRS approach in combination with a toxicity prediction model and a cost-effectiveness analyses is a promising basis for a decision support tool for the implantation of either a hydrogel spacer or a rectum balloon implant. 


\section{Introduction}

For patients with prostate cancer, intensity modulated radiotherapy (IMRT) is one of the curative treatment options. Dose-escalation has revealed to improve tumor control and overall survival in intermediate and high-risk prostate cancer ${ }^{[1-3]}$. However, this also leads to increased risk of gastro-intestinal (GI) toxicity which negatively impacts the quality of life $(\mathrm{Q} O \mathrm{~L})^{[4,5]}$. The proximity between the prostate and the anterior rectal wall causes part of the high radiation-dose region (>90\%) to overlap with the rectum, the primary organ at risk (Figure 4.1A).

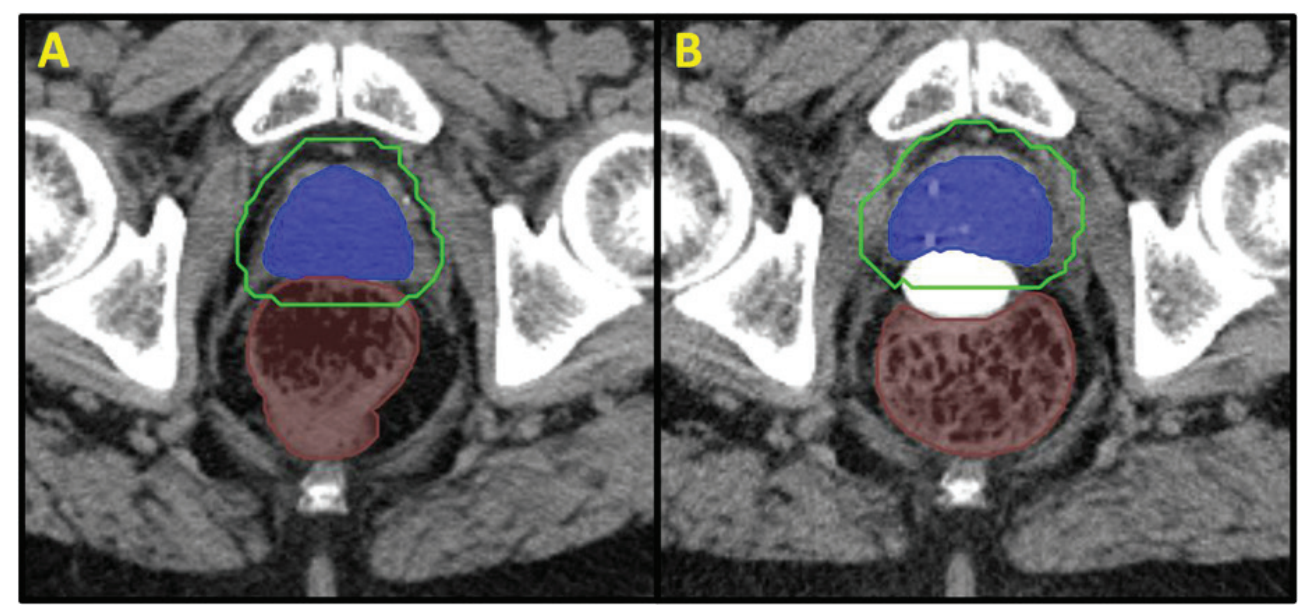

Figure 4.1 - Flowchart (A) The prostate (blue) and rectum (brown) before placement of an Internal Rectum Spacer and (B) after the placement of the Spacer. The contour is the isodose at $90 \%$ of the target dose.

To spare the rectum, two commercially available implantable rectum spacers (IRS) have been developed: one type based on polyethylene glycol hydrogels (SpaceOAR $^{\text {TM }}$ System, Augmenix Inc. $)^{[6]}$ and the other is a biodegradable saline-filled rectal balloon implant (BioProtect $L t d$, Israel) ${ }^{[7,8]}$. These devices have been designed to increase the distance between the anterior rectal wall and the prostate, and pushing the rectal wall out of the high-dose region (Figure 4.1B). Several studies have shown that this technique results in lower dose within the rectum, resulting in lower toxicity levels ${ }^{[9,10]}$. However, the IRS has not shown significant improvements for all patients, and is a costly and invasive procedure ${ }^{[11-13]}$.

To identify the patients that are expected to benefit most from an IRS, we developed a novel method to explore a priori the benefit of the IRS. The method simulates an IRS through the use of deformation fields and predicts the geometric result of an IRS before the start of treatment: a so-called virtual implantable rectum spacer ( $\mathrm{V}-\mathrm{IRS})$. This results in a comparison between computed tomography (CT) scans of the patient with a V-IRS (prior to the start of the treatment), and one 
without IRS. To this end, a comparison of dose gain distributions, predicted normal tissue complication probabilities (NTCP) and a cost-effectiveness (CE) analysis between these two treatment plans can be performed (Figure 4.2). The developed method is based on the multifactorial decision support system developed $\mathrm{in}^{[14]}$ and may be used to support the decision on the implantation of an IRS for individual patients $^{[15,16]}$. In this study, we evaluated the V-IRS' performance in helping the decision support to implant an IRS or not for patients with prostate cancer. We did this by comparing the V-IRS to the real IRS on the three different levels: dose, NTCP, and CE.

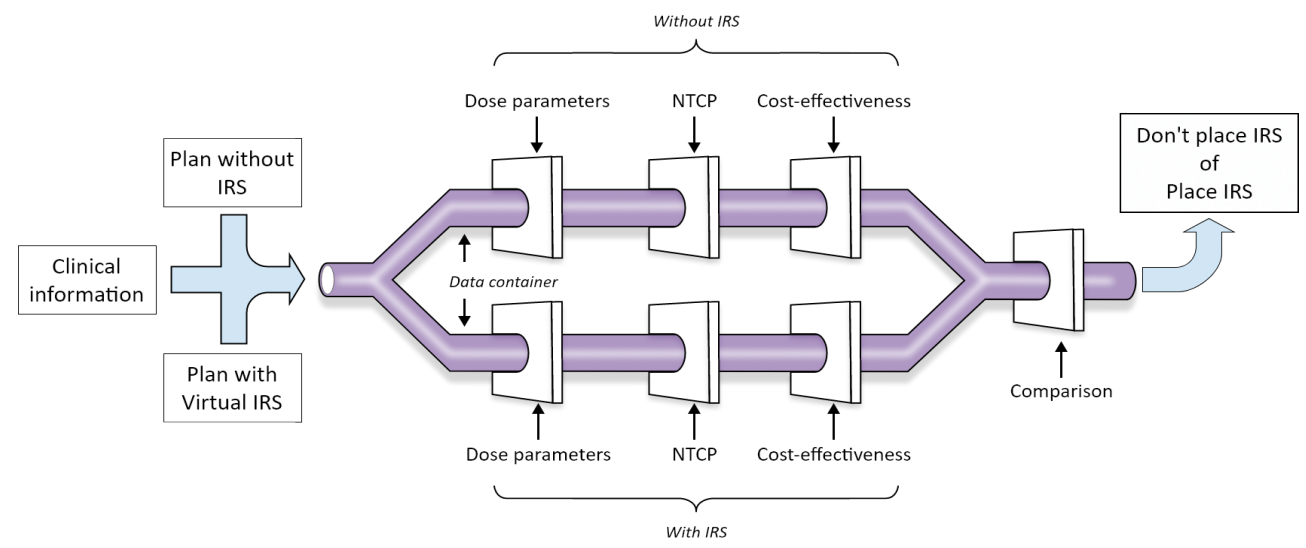

Figure 4.2 - An overview of the decision support system for which the virtual internal rectum spacer (IRS) would be a basis. The image shows the input to be a treatment plan for a patient without the IRS, a treatment plan for a patient with the virtual IRS and relevant clinical information. The input data are then split into two different pipelines: one for without IRS and one for with IRS, and a three level analyses is performed. The two pipelines come together in a third pipeline, where comparison is made which can help support the decision to place a spacer or not. NTCP = normal tissue complication probability.

\section{Material and methods}

\section{Patient characteristics}

This study included 23 patients with localized prostate cancer who have signed informed consent. We placed a rectal balloon implant (RBI) in 15 of these patients and 8 patients had a hydrogel IRS implanted. All patients were included after approval by the local ethics committee.

\section{Image acquisition and organ delineation}

Each patient was scanned in supine position using standard CT imaging. The patients with a hydrogel IRS all underwent two CT scans: one prior to the IRS implantation, and one several days after implantation. The slice thickness of these scans was $5 \mathrm{~mm}$, with 
a pixel size of approx. $1 \mathrm{~mm}$. Of the 15 patients who received a RBI, 8 were scanned prior to implantation as well as several days after, and 7 only after implantation. The slice thickness for these scans was $3 \mathrm{~mm}$ with a pixel size of $1 \mathrm{~mm}$.

The clinical target volume (CTV) in the patients who received a RBI was defined as the prostate only, and for the patients with a hydrogel it was defined as the prostate with seminal vesicles, according the risk of involvement of the seminal vesicles.

The planning target volume (PTV) was obtained by expanding the CTV by $6 \mathrm{~mm}$ in all directions to account for geometrical uncertainties (daily setup variability and internal organ motion).

\section{V-IRS}

To predict the geometrical changes which occur after implantation of an IRS, a virtual CT image was created using image deformation.

The precise shape of an IRS after implementation varied from patient to patient, which is why a model of an IRS was used as the basis for the deformation. The model of the RBI was obtained using the CT scans of 7 of the patients with RBI. The method used is visualized in Supplementary Figure 1.

The model IRS was inserted into the CT image by applying a deformation field, shown in Supplementary Figure 2, to a CT image of a patient prior to the implantation of an IRS. Several assumptions were made during the development of the deformation field. First, the deformation was assumed to only take place along the direction in which the IRS inflates. Though a small amount of deformation took place along the other axes, this effect was assumed minimal compared to the inflation direction. Second, the deformation was assumed to affect up to $2 \mathrm{~cm}$ of tissue. After this, the deformation fades due to compression. Last, due to the stiffness of the prostate and the rectum, the organs didn't align perfectly around the IRS. This effect was simulated using a parabolic distribution. The field was applied to the CT image and contours using the REGGUI software for MATLAB ${ }^{\circledR[17]}$, placing the center of the field between the middle of the prostate and the rectum. A comparison between the shape of the real IRS and organs is compared to the shape of the V-IRS and organs in Supplementary Figure 3.

\section{Dose distribution}

A certified technician applied a treatment plan on the CT scan prior to IRS implementation, with IRS, and on the virtual image. All treatment plans were designed for dose delivery with a volumetric modulated arc technique with $10 \mathrm{MV}$ photon beams (Eclipse Version ICD-10, Varian Medical Systems Inc., Palo Alto, USA). The treatment plans included 28 fractions of 2.5 Gray (Gy), resulting in a total dose of $70 \mathrm{~Gy}{ }^{[18]}$. The equivalent $2 \mathrm{~Gy}$ dose (EQD2) for this type of plan is $77 \mathrm{~Gy}{ }^{[19]}$, for an $\alpha / \beta$ ratio of $3 \mathrm{~Gy}$ for late rectal toxicity ${ }^{[20]}$, and 80 Gy with an $\alpha / \beta$ ratio of 1.5 Gy for a prostate tumor. 
The Dose Volume Histogram (DVH) of the dose within the rectum was used for dose comparison between the plan without IRS to the plan with IRS. To test the $\mathrm{V}$-IRS, three treatment plans were made for each patient; one on the scan before IRS insertion, one on the scan with the IRS, and one on the scan with the V-IRS. To check that all plans were comparable, the equivalent uniform dose (EUD) in the CTV was calculated for each plan.

\section{Toxicity model}

To indicate whether the IRS results in significant improvement in GI toxicity for a given patient, the DVH alone is not sufficient. Clinical differences between patients result in variations in predicted toxicity even when the dose distribution is similar. In order to predict the $\mathrm{Gl}$ toxicity resulting from the treatment plan in combination with certain clinical parameters, a validated NTCP model was used (Table 4.1). The chosen model is a multivariate logistic regression model that uses the mean rectal dose, the percentage of the volume of the rectum receiving more than 75 Gy (V75) and several clinical parameters as predictors for late rectal bleeding ${ }^{[21,22]}$. The concordance indices for this model were 0.62 and is the same NTCP model used $\mathrm{in}^{[11]}$. The IRS is expected to improve the NTCP predicted by this model, because the IRS increases the distance between the rectum and the prostate, thus reducing the $\mathrm{V} 75$ in the rectum.

Table 4.1 - Overview of the NTCP models used.

\begin{tabular}{l|ll} 
G2-G3 acute rectal bleeding $(\mathbf{N}=\mathbf{1 , 1 3 2})$ & Coefficient & SE \\
\hline Constant & -2.489 & 0.5243 \\
Use of anticoagulants (0/1) & -0.4702 & 0.2034 \\
Diabetes (0/1) & 0.2445 & 0.2543 \\
Hemorrhoids (O/1) & 0.4066 & 0.1796 \\
Irradiation of pelvic nodes (0/1) & 0.4455 & 0.2821 \\
Hormonal therapy (0/1) & -0.4252 & 0.2079 \\
Mean rectal dose (Gy) & 0.0339 & 0.0096 \\
\hline G2-G3 late rectal bleeding (N= 718) & Coefficient & SE \\
\hline Constant & -3.5082 & 0.7000 \\
G2-G3 acute rectal bleeding (\%) & 0.0258 & 0.0173 \\
Prior abdominal surgery & 0.7465 & 0.4175 \\
V75 in rectum (\%) & 0.0571 & 0.0215 \\
\hline
\end{tabular}

G2-G3: grade 2-grade 3 standard RTOG/EORTC scores, RTOG: Radiation therapy oncology group, EORTC: European organization for research and treatment of cancer, Gy: Gray, V75: volume percentage receiving more than $75 \mathrm{~Gy}$, SE: standard error 


\section{Cost-effectiveness model}

The system is expanded with a published Markov model that analyses the CE of the implantation of the IRS by comparing the gain from the IRS in quality of life with the increased costs associated with the $\mathrm{IRS}^{[13]}$. The Markov model calculates the incremental net monetary benefit (iNMB) between the two treatment options: IMRT with IRS (IMRT+IRS) and IMRT without IRS (IMRT-IRS). The NMB is obtained by multiplying the number of quality adjusted life years (QALY) with a willingness to pay (WTP) threshold and subtracting the treatment costs. The iNMB is obtained by subtracting the NMB of IMRT-IRS from the NMB of IMRT+IRS. If the iNMB is positive, the implantation of an IRS is considered cost-effective. In this study a WTP threshold of $€ 80,000$ is used, which is the informal ceiling ratio for a high burden of disease in the Netherlands ${ }^{[23]}$. The WTP varies strongly per country ( $£ 20,000-£ 30,000$ in the UK and $\$ 50,000-\$ 100,000$ in the USA), and is a variable input parameter in the model. Whether or not the IRS was effective or not in a given patient strongly depends on the decrease in NTCP resulting from the IRS. A reduced NTCP results in more QALY's, but also lower follow-up costs resulting from toxicity.

\section{Statistics}

We used two-tailed Wilcoxon signed rank tests to determine whether the differences between the plans without IRS and the plans with IRS were significant. P-values of less than 0.05 were considered significant.

\section{Results}

\section{Rectum Balloon Implant results}

The implantation of a RBI resulted in a minor reduction of the mean rectal dose: 1.5 Gy[-14.9-12.4] average ( $p=0.383)$ for the RBI and 5 Gy[2.3-8.4] for the V-IRS ( $p$ $=0.008$ ) (Table 4.2). Large differences were observed in rectal volumes in IMRT+IRS and IMRT+V-IRS: in one patient the rectal volume before RBI implantation was $215.5 \mathrm{~cm}^{3}$, and $33.1 \mathrm{~cm}^{3}$ after RBI implantation. The EUD in the CTV for all these plans was between 79 and $80.5 \mathrm{~Gy}$.

The mean reduction in $\mathrm{V} 75$ was $3.2 \%[0.4-8.6](p=0.008)$ resulting from the RBI and 3.2\% [1.0-7.8] from the V-IRS. The largest difference between the V75 of the plans on the CT's after the implantation of an RBI and those with a V-IRS was $0.9 \%$. 
A NTCP comparison between RBI and V-IRS

B NTCP comparison between HGS and V-IRS

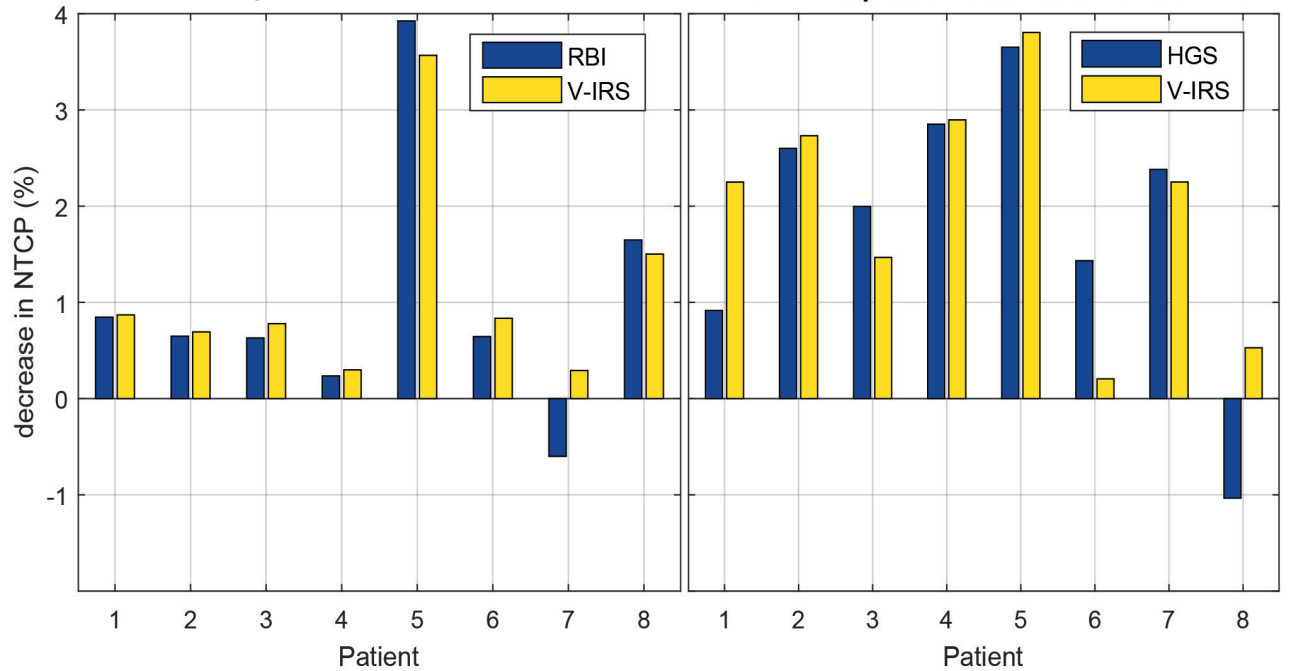

Figure 4.3 - The decrease in normal tissue complication probability (iNTCP) as a result of the implantable rectum spacer (IRS). The results for the rectum balloon implants (RBI) are shown in $A$, and those for the Hydrogel spacer in $B$. These decreases are compared to those caused by the virtual IRS (V-IRS).

A NMB comparison between RBI and V-IRS

B NMB comparison between HGS and V-IRS

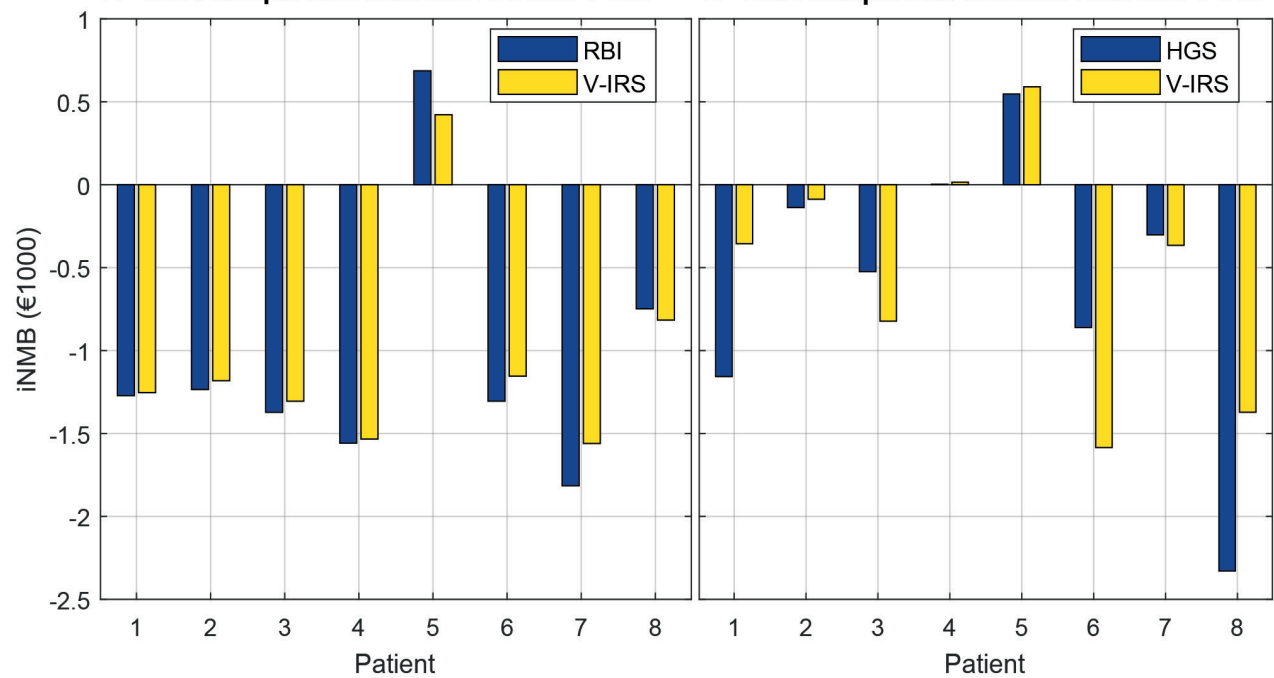

Figure 4.4 - The incremental net monetary benefits for each of the patients with a Rectum balloon implant in A, and with a Hydrogel Spacer in B. If the iNMB is negative, the implantation of an IRS is not cost-effective. 
The NTCP reduction resulting from the RBI was on average 1.0\% [-0.6-3.9], and $1.1 \%$ [0.3-3.5] resulting from the V-IRS. The decrease in NTCP resulting from the $\mathrm{RBI}$ and from the V-IRS is shown in Figure 4.3A. Note that the NTCP for patient 7 was lower than before the IRS was implanted. This resulted in an average iNMB of - $€ 1158$ and - $€ 1032$ for the RBI and the $V$-IRS respectively, showing that the implantation of an IRS is not cost-effective when performed on all 8 patients. The iNMB's for each of the patients that received a RBI are shown in Figure 4.4A. For all these patients, the V-IRS predicts correctly whether or not the implantation of an IRS is cost-effective. For one patient among these eight the implantation is expected to be cost-effective.

\section{Hydrogel results}

The mean rectal dose was reduced by $5.6 \mathrm{~Gy}[-7.8-7.0]$ after the implantation of a hydrogel IRS ( $p=0.055)$, and by 6.3 Gy [1.8-4.0] with the V-IRS ( $p=0.008$ ) (Table 4.2). For this set of patients, large differences in rectal volume were found as well, one with $263 \mathrm{~cm}^{3}$ before the implantation of the hydrogel IRS and $156 \mathrm{~cm}^{3}$ after. The implantation of the hydrogel IRS resulted in a mean $V 75$ reduction of $5.2 \%$ [-1.7-9.8] $(p=0.0156)$, and the V-IRS predicted a reduction of 5.4\% [0.9-9.8] ( $p=$ 0.008). The largest difference between the V75 of the plans on the CT's after the implantation of a hydrogel and those with a V-IRS was 3.7\%. The EUD in the CTV for all these plans was between 79.5 and 81 Gy.

The NTCP was 1.8\% [-1-3.8] lower, on average, after the implantation of a hydrogel IRS than before, and 1.9\% [0.4-3.9] lower with V-IRS. The decrease in NTCP resulting from the Hydrogel and from the $\mathrm{V}$-IRS is shown in Figure 4.3B. Note that the NTCP for patient 8 was lower than before the IRS was implanted. The resulting iNMB was- $€ 669$ and- $€ 567$ for the hydrogel IRS and the V-IRS, respectively, showing that the implantation of the hydrogel is also not cost-effective when applied to all patients. Figure 4.4B shows the iNMB's for each of the patients with a hydrogel spacer. For this set of patients the V-IRS classifies the cost-effectiveness correctly when compared to the real IRS. Patient 4 and 5 appear to be cost-effective, though patient 4 with only a small gain, and patient 2 is close to cost-effective. 
Table 4.2 - Overview of results for the Rectum Balloon implants (RBI) and the virtual implantable rectum spacer ( $V$-IRS).

\begin{tabular}{|c|c|c|c|}
\hline & Before RBI & After RBI & $V$-IRS \\
\hline $\begin{array}{l}\text { Mean Rectal Dose (EQD2 Gy) } \\
\text { [range] }\end{array}$ & $\begin{array}{l}20.8 \\
{[10.5-39.9]}\end{array}$ & $\begin{array}{l}19.3 \\
{[10.2-31.3]}\end{array}$ & $\begin{array}{l}15.8^{*} \\
{[7.9-32.9]}\end{array}$ \\
\hline $\begin{array}{l}\text { V75 in Rectum (\%) } \\
\text { [range] }\end{array}$ & $\begin{array}{l}3.4 \\
{[1.1-9.0]}\end{array}$ & $\begin{array}{l}0.2^{*} \\
{[0-0.7]}\end{array}$ & $\begin{array}{l}0.2^{*} \\
{[0-1.2]}\end{array}$ \\
\hline $\begin{array}{l}\text { NTCP (\%) } \\
\text { [range] }\end{array}$ & $\begin{array}{l}4.9 \\
{[3.8-8.5]}\end{array}$ & $\begin{array}{l}3.9 \\
{[3.5-4.8]}\end{array}$ & $\begin{array}{l}3.8 \\
{[3.2-5.2]}\end{array}$ \\
\hline $\begin{array}{l}\text { iNMB } \\
\text { [range] }\end{array}$ & - & $\begin{array}{l}-€ 1158 \\
{[-€ 2049-€ 481]}\end{array}$ & $\begin{array}{l}-€ 1032 \\
{[-€ 1497-€ 328]}\end{array}$ \\
\hline \multicolumn{2}{|r|}{ Before Hydrogel } & After Hydrogel & V-IRS \\
\hline $\begin{array}{l}\text { Mean Rectal Dose (EQD2 Gy) } \\
\text { [range] }\end{array}$ & $\begin{array}{l}31.9 \\
{[25.2-41.0]}\end{array}$ & $\begin{array}{l}26.3 \\
{[18.9-32.9]}\end{array}$ & $\begin{array}{l}25.6^{*} \\
{[19.8-33.8]}\end{array}$ \\
\hline $\begin{array}{l}\text { V75 in Rectum (\%) } \\
\text { [range] }\end{array}$ & $\begin{array}{l}6.2 \\
{[1.9-9.8]}\end{array}$ & $\begin{array}{l}1.0^{*} \\
{[0-3.6]}\end{array}$ & $\begin{array}{l}0.8^{*} \\
{[0-4.7]}\end{array}$ \\
\hline $\begin{array}{l}\text { NTCP (\%) } \\
\text { [range] }\end{array}$ & $\begin{array}{l}6.2 \\
{[4.8-8.2]}\end{array}$ & $\begin{array}{l}4.4 \\
{[3.6-5.8]}\end{array}$ & $\begin{array}{l}4.3 \\
{[3.7-4.8]}\end{array}$ \\
\hline $\begin{array}{l}\text { iNMB } \\
\text { [range] }\end{array}$ & - & $\begin{array}{l}-€ 669 \\
{[-€ 2308-€ 526]}\end{array}$ & $\begin{array}{l}-€ 567 \\
{[-€ 1525-€ 542]}\end{array}$ \\
\hline
\end{tabular}

RBI: Rectal Balloon implant, V-IRS: Virtual Implantable Rectum Spacer, EQD2: Equivalent 2 Gy Dose, V75: Volume percentage receiving at least 75 Gy, NTCP: Normal tissue complication probability, iNMB: incremental Net Monetary Benefit.

* Two-tailed Wilcoxon signed rank test: significant difference in dose at 5\% from the plan before hydrogel 


\section{Discussion}

In this study, we have developed a V-IRS which can be used to support the decision for the implantation of an IRS for a specified patient or not.

Our method has two main strengths. The first is that a CT of a patient with an IRS can be generated and made available for treatment planning purposes without the real implantation of the IRS itself. This means that the benefit of the IRS for the specified patient can be estimated without having to put the patient through the discomfort of the operation, and without the additional costs of the placement of the IRS. The second is that instead of only looking at the improved dose reduction by implanting the IRS, the NTCP reduction and the CE are also taken into consideration. This allows for a decision based not only on dose reduction, but also on the health gain and the costs associated with the health gain.

The V-IRS gives a good approximation of NTCP and of the cost-effectiveness when compared to an IRS for both commercial systems: the RBI and the hydrogel IRS. The differences between the health gain predicted by the $\mathrm{V}$-IRS and achieved by the RBI were small for 7 of the 8 patients. For the hydrogel these differences were larger, especially when the NTCP was smaller than $2.5 \%$. However, at higher NTCP values, the V-IRS made more accurate predictions. On CE level, the V-IRS performed well: each time the real IRS was expected not to be cost-effective, the $V$-IRS had the same conclusion, and vice versa. The differences in iNMB between the $\mathrm{V}$-IRS and the RBI were small, showing that the $\mathrm{V}$-IRS is an excellent approximation of the RBI. The differences in iNMB between the hydrogel IRS and the $\mathrm{V}$-IRS were larger, but not enough to cause a classification error.

The V-IRS was based on the RBI, which, as a closed system, inflates to a predetermined shape, which is not the case for the hydrogel IRS. This could cause errors in the predicted iNMB for the hydrogel spacer, but due to the variability of the shape of the hydrogel, a V-IRS based on a hydrogel is not likely to be more predictive than the one used in this study. However, the volume of the hydrogel was often lower than the V-IRS, possibly overestimating its effectiveness. This could be solved by decreasing the V-IRS volume when applied to hydrogels.

The results presented in this study showed that the patients who were to be implanted with a hydrogel spacer had higher NTCP's than the patients to be implanted with a RBI. This was because all patients in the hydrogel spacer group had the seminal vesicles included in the CTV, while the patients with in the RBI group didn't. This is kept consistent with the V-IRS, and did not visibly influence its performance.

One of the main limitations of the study was the small amount of patients included. Though they were sufficient for a first proof of concept, a more elaborate study including a larger amount of patients is required for validation of the method. 
Further, the deformation field used to create the images with the V-IRS was arbitrarily chosen, and did not succeed in accurately predicting the deformation caused by the IRS. These differences in precise shape did not impact the performance on NTCP level because at very low dose levels the NTCP is impacted more strongly by clinical factors than dose, so as long as the spacing caused by the IRS was sufficient, the predictions were precise enough for classification. However, better deformation predictions would lead to more accurate classifications. Image registration could be used to develop a deformation field once a larger amount of patients becomes available which could be updated every time a new patient is treated.

Next, the mean rectal dose has been shown to be a predictor for acute rectal bleeding, which, on its turn, is a predictor for late rectal bleeding (consequential late effect). Although the mean rectal dose is dependent on the overlap between the rectal wall and the high-dose region, it is mainly affected by the rectum volume, which varies strongly ${ }^{[24,25]}$. This change in rectal volume cannot be predicted by the model, and might influence the decision on whether or not to place an IRS. For 2 patients, patient 7 with a RBI, and patient 8 with a hydrogel, this even led to an increase in predicted NTCP. Future works should include models that use the dose surface histogram (DSH), rather than the DVH in the rectum, as this is much less affected by the rectal volume ${ }^{[26]}$.

During this study the only NTCP taken into account was for late rectal bleeding. Late fecal incontinence, erectile dysfunction, and urinary symptoms are not calculated, but do have an effect on the patients QoL. An appropriate expansion to the system would be to include NTCP models for these types of toxicities. The possible complications after placement of the IRS (pain, rectal perforation) were not taken into account, as the risk of these factors is low $(<5 \%)^{[27]}$, and are not yet fully described. Furthermore, the nomograms on which the NTCP model is based are from 2002-2004, where now outdated dose delivery techniques were conformal. The performance of a decision support system based on a V-IRS is partially dependent on the accuracy of the NTCP models. This must be taken into account during further development of the system, by using newer models when these become available.

The current model is isodosimetric which shows the toxicity for both the CT images before the IRS and after the IRS assuming both receive the same dose within the target volume. Future studies should include an analyses of an isotoxic version of the model, which would analyze how much dose escalation is possible while still maintaining low NTCP values for each of the different situations. Including genetic markers into the NTCP model would also improve the decision support on whether or not to place an IRS in a prostate cancer patient ${ }^{[28]}$. 


\section{Conclusion}

The implantation of an IRS is not cost-effective for all patients, so individual patient assessment is needed. The V-IRS approach in combination with a NTCP model and a CE analysis can serve as the basis for a decision support system for the implantation of either a hydrogel IRS or a rectum balloon implant.

\section{Acknowledgements}

Marlies Lendfers is gratefully acknowledged for her help in developing the treatment plans used in this study.

Authors acknowledge financial support from ERC advanced grant (ERCADG-2015, $n^{\circ} 694812$ - Hypoximmuno). Authors also acknowledge financial support from the EU 7th framework program (REQUITE - $n^{\circ}$ 601826), SME Phase 2 (RAIL - $n^{\circ} 673780$ ), EUROSTARS (DART), the European Program H2020-2015-17 (PREDICT- ITN- $\mathrm{n}^{\circ}$ 766276) and Interreg V-A Euregio Meuse-Rhine ("Euradiomics"). 


\section{References}

1. Zelefsky, M.J., X. Pei, J.F. Chou, M. Schechter, M. Kollmeier, B. Cox, et al., Dose escalation for prostate cancer radiotherapy: predictors of long-term biochemical tumor control and distant metastases-free survival outcomes. Eur Urol, 2011. 60(6): p. 1133-9.

2. Kalbasi, A., J. Li, A. Berman, S. Swisher-McClure, M. Smaldone, R.G. Uzzo, et al., Dose-Escalated Irradiation and Overall Survival in Men With Nonmetastatic Prostate Cancer. JAMA Oncol, 2015. 1(7): p. 897-906.

3. Ben G.L.Vanneste, E.J.V.L., Emile N. van Lin, Joep G.H. van Roermund, and Philippe Lambin, Prostate Cancer Radiation Therapy: What Do Clinicians Have to Know? BioMed Research International, 2016. 2016: p. 14.

4. Vanneste, B.G., L. Van De Voorde, R.J. de Ridder, E.J. Van Limbergen, P. Lambin,E.N. van Lin, Chronic radiation proctitis: tricks to prevent and treat. Int J Colorectal Dis, 2015. 30(10): p. 1293-303.

5. Glass, A.S., J.E. Cowan, M.J. Fuldeore, M.R. Cooperberg, P.R. Carroll, S.A. Kenfield, et al., Patient demographics, quality of life, and disease features of men with newly diagnosed prostate cancer: trends in the PSA era. Urology, 2013. 82(1): p. 60-5.

6. Pinkawa, M., N.E. Corral, M. Caffaro, M.D. Piroth, R. Holy, V. Djukic, et al., Application of a spacer gel to optimize three-dimensional conformal and intensity modulated radiotherapy for prostate cancer. Radiother Oncol, 2011. 100(3): p. 436-41.

7. Wolf, F., C. Gaisberger, I. Ziegler, E. Krenn, P. Scherer, S. Hruby, et al., Comparison of two different rectal spacers in prostate cancer external beam radiotherapy in terms of rectal sparing and volume consistency. Radiother Oncol, 2015. 116(2): p. 221-5.

8. Gez, E., S. Cytron, R. Ben Yosef, D. London, B.W. Corn, S. Alani, et al., Application of an interstitial and biodegradable balloon system for prostate-rectum separation during prostate cancer radiotherapy: a prospective multi-center study. Radiat Oncol, 2013. 8: p. 96.

9. Hamstra, D.A., N. Mariados, J. Sylvester, D. Shah, L. Karsh, R. Hudes, et al., Continued Benefit to Rectal Separation for Prostate Radiation Therapy: Final Results of a Phase III Trial. Int J Radiat Oncol Biol Phys, 2016.

10. Mariados, N., J. Sylvester, D. Shah, L. Karsh, R. Hudes, D. Beyer, et al., Hydrogel Spacer Prospective Multicenter Randomized Controlled Pivotal Trial: Dosimetric and Clinical Effects of Perirectal Spacer Application in Men Undergoing Prostate Image Guided Intensity Modulated Radiation Therapy. Int J Radiat Oncol Biol Phys, 2015. 92(5): p. 971-7.

11. Vanneste, B.G., A.L. Hoffmann, E.N. van Lin, L. Van De Voorde, M. Pinkawa,P. Lambin, Who will benefit most from hydrogel rectum spacer implantation in prostate cancer radiotherapy? A model-based approach for patient selection. Radiother Oncol, 2016.

12. Hutchinson, R.C., V. Sundaram, M. Folkert,Y. Lotan, Decision analysis model evaluating the cost of a temporary hydrogel rectal spacer before prostate radiation therapy to reduce the incidence of rectal complications. Urol Oncol, 2016. 34(7): p. 291 e19-26.

13. Ben G.L. Vanneste, M.P.J., Lien Van De Voorde, Emile N. van Lin,J.J.L. Kees van de Beek, Bram L. Ramaekers, Philippe Lambin, Spacers in radiotherapy treatment of prostate cancer: Is reduction of toxicity cost-effective? Radiotherapy and Oncology, 2015. 114(2015): p. 276-281.

14. Cheng, Q., E. Roelofs, B.L. Ramaekers, D. Eekers, J. van Soest, T. Lustberg, et al., Development and 
evaluation of an online three-level proton vs photon decision support prototype for head and neck cancer- Comparison of dose, toxicity and cost-effectiveness. Radiother Oncol, 2016. 118(2): p. 281-5.

15. Lambin, P., E. Roelofs, B. Reymen, E.R. Velazquez, J. Buijsen, C.M. Zegers, et al., 'Rapid Learning health care in oncology' - an approach towards decision support systems enabling customised radiotherapy'. Radiother Oncol, 2013. 109(1): p. 159-64.

16. Lambin, P., R.G. van Stiphout, M.H. Starmans, E. Rios-Velazquez, G. Nalbantov, H.J. Aerts, et al., Predicting outcomes in radiation oncology--multifactorial decision support systems. Nat Rev Clin Oncol, 2013. 10(1): p. 27-40.

17. Janssens, G., L. Jacques, J. Orban de Xivry, X. Geets,B. Macq, Diffeomorphic registration of images with variable contrast enhancement. Int J Biomed Imaging, 2011. 2011: p. 891585.

18. Kupelian, P.A., T.R. Willoughby, C.A. Reddy, E.A. Klein,A. Mahadevan, Hypofractionated intensitymodulated radiotherapy (70 Gy at 2.5 Gy per fraction) for localized prostate cancer: Cleveland Clinic experience. Int J Radiat Oncol Biol Phys, 2007. 68(5): p. 1424-30.

19. Barton, M., Tables of equivalent dose in 2 Gy fractions: a simple application of the linear quadratic formula. Int J Radiat Oncol Biol Phys, 1995. 31(2): p. 371-8.

20. Marzi, S., B. Saracino, M.G. Petrongari, S. Arcangeli, S. Gomellini, G. Arcangeli, et al., Modeling of alpha/ beta for late rectal toxicity from a randomized phase II study: conventional versus hypofractionated scheme for localized prostate cancer. J Exp Clin Cancer Res, 2009. 28: p. 117.

21. Valdagni, R., M.W. Kattan, T. Rancati, C. Yu, V. Vavassori, G. Fellin, et al., Is it time to tailor the prediction of radio-induced toxicity in prostate cancer patients? Building the first set of nomograms for late rectal syndrome. Int J Radiat Oncol Biol Phys, 2012. 82(5): p. 1957-66.

22. Valdagni, R., T. Rancati, C. Fiorino, G. Fellin, A. Magli, M. Baccolini, et al., Development of a set of nomograms to predict acute lower gastrointestinal toxicity for prostate cancer 3D-CRT. Int J Radiat Oncol Biol Phys, 2008. 71(4): p. 1065-73.

23. Sensible and sustainable care W.a.S. the Council for Public Health and Health Care to the Minister of Health, Editor. 2006: Zoetermeer.

24. de Crevoisier, R., S.L. Tucker, L. Dong, R. Mohan, R. Cheung, J.D. Cox, et al., Increased risk of biochemical and local failure in patients with distended rectum on the planning $\mathrm{CT}$ for prostate cancer radiotherapy. Int J Radiat Oncol Biol Phys, 2005. 62(4): p. 965-73.

25. Hoogeman, M.S., M. van Herk, J. de Bois,J.V. Lebesque, Strategies to reduce the systematic error due to tumor and rectum motion in radiotherapy of prostate cancer. Radiother Oncol, 2005. 74(2): p. 177-85.

26. Meijer, G.J., M. van den Brink, M.S. Hoogeman, J. Meinders,J.V. Lebesque, Dose-wall histograms and normalized dose-surface histograms for the rectum: a new method to analyze the dose distribution over the rectum in conformal radiotherapy. Int J Radiat Oncol Biol Phys, 1999. 45(4): p. 1073-80.

27. Vanneste, B.G., K. van De Beek, L. Lutgens,P. Lambin, Implantation of a biodegradable rectum balloon implant: tips, Tricks and Pitfalls. Int Braz J Urol, 2017. 43.

28. Fachal, L., A. Gomez-Caamano, G.C. Barnett, P. Peleteiro, A.M. Carballo, P. Calvo-Crespo, et al., A threestage genome-wide association study identifies a susceptibility locus for late radiotherapy toxicity at 2q24.1. Nat Genet, 2014. 46(8): p. 891-4. 


\section{Supplementary Material}
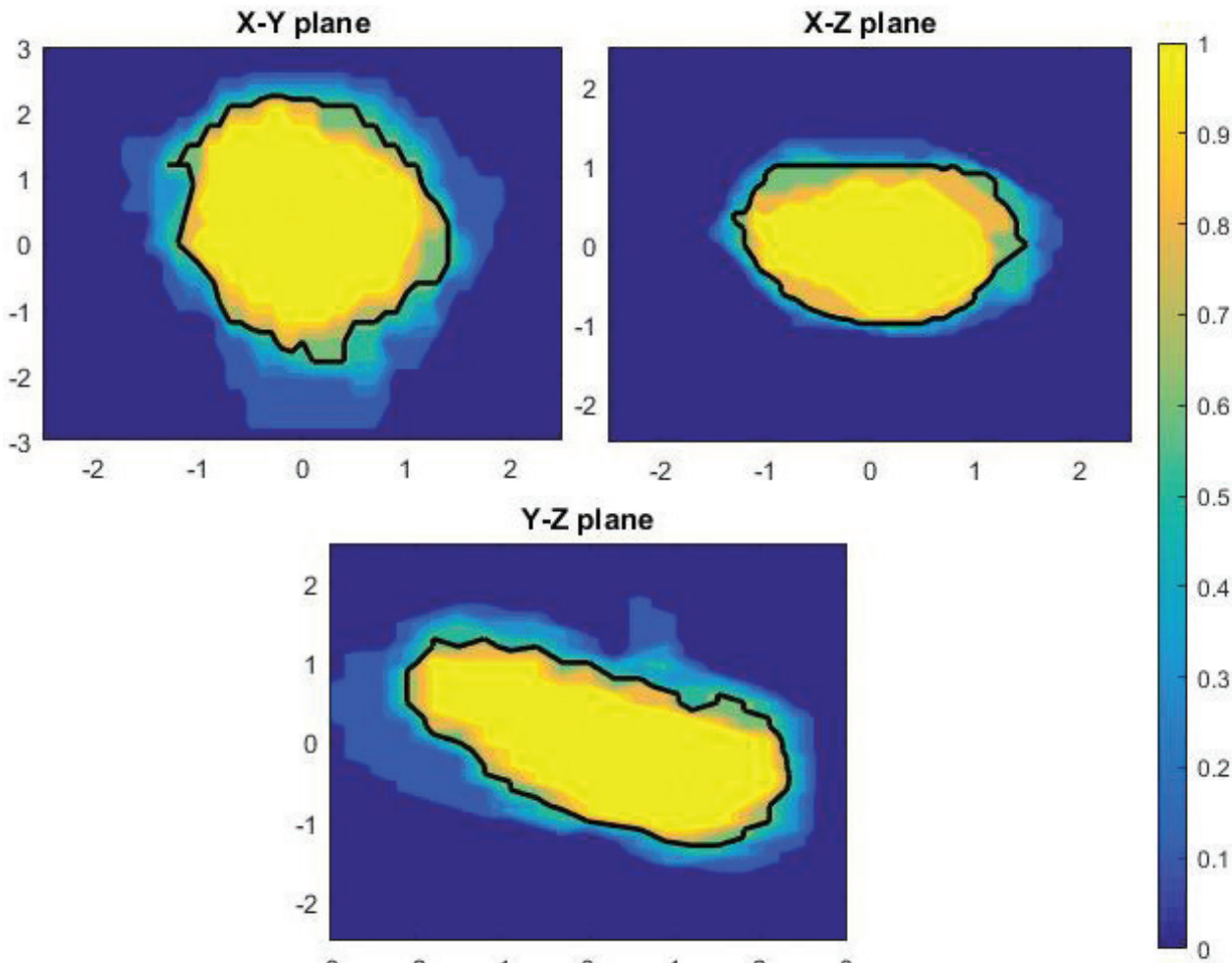

Supplementary Figure S4.1 - Seven delineations of Implantable rectum spacers (IRS) were used to create a probability map for the shape of the rectal balloon implant. At value 1, all IRS's overlap, at value 0, no delineations of IRS's were found at all. The threshold for the model IRS was set at 0.6 and is indicated by the black contour. 


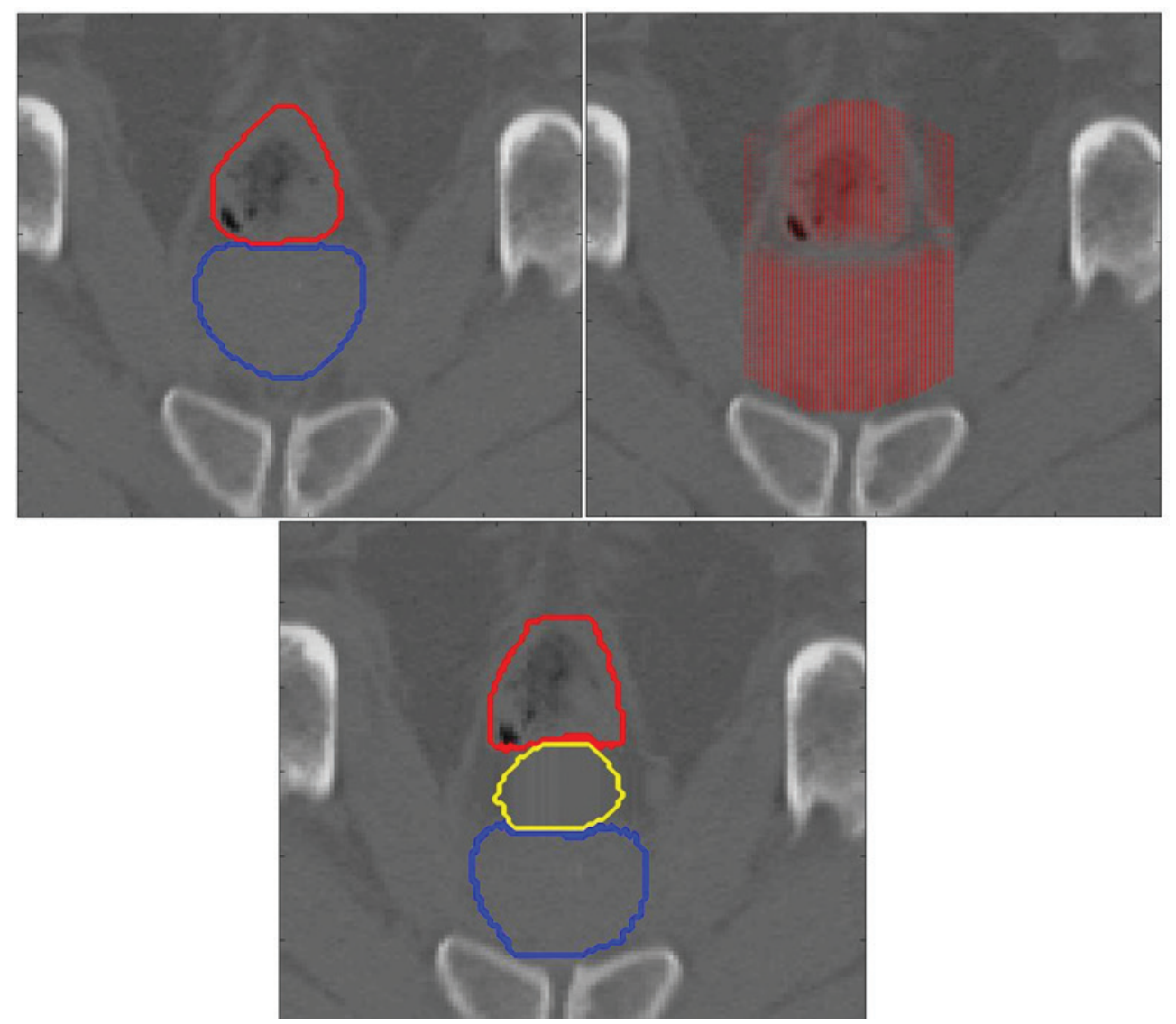

Supplementary Figure S4.2 - The deformation field assumed only deformation along the axis through both the prostate and the rectum. After $2 \mathrm{~cm}$ the deformation field is 0 . The spacer is assumed to have deformation effect in a hyperbolic distribution along the other axes. The top left image shows the prostate and rectum before deformation. The top right shows the deformation field. The bottom image shows the result of the deformation field. 

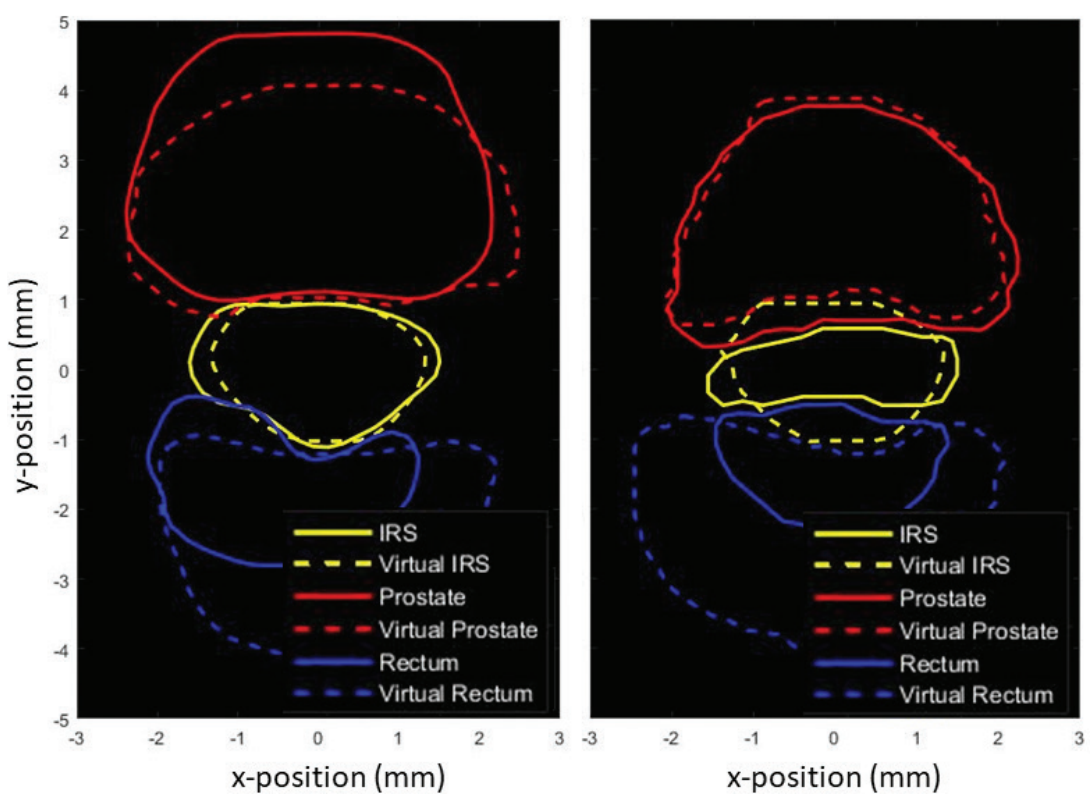

Supplementary Figure S4.3-An overlap of the structures one of the images with the real implantable rectum spacer (IRS) and with the virtual IRS. On the left side the structures of the real image are that of a rectum balloon implant, on the right side the real structures are of a hydrogel spacer.

Supplementary Table S4.1: An overview of the constraints set during treatment planning (given dose)

\begin{tabular}{l|ll} 
Structure & Constraints & V77 $<3 \%$ \\
\hline Planning target volume & $\mathrm{V} 70>95 \%$ & \\
Rectum volume & $\mathrm{V} 70=0 \%$ & Mean dose $<40$ gy \\
Anal canal volume & $\mathrm{V} 74=0 \%$ & V65 $<20 \%$ \\
Anorectum volume & $\mathrm{V} 54<50 \%$ & \\
Bladder volume & $\mathrm{V} 70=0 \%$ &
\end{tabular}

Gy: gray, vxx: percentage of volume receiving more than $x x$ gy 
Supplementary Table S4.2: Overview of the distances between the rectum and the prostate at mid-prostate level and the PTV overlap with the rectum after placement of an RBI or hydrogel spacer and after application of the $\mathrm{V}$-IRS.

\begin{tabular}{|c|c|c|c|}
\hline Property & $R B I$ & $V-I R S$ & P-Value \\
\hline $\begin{array}{l}\text { Median Distance }(\mathrm{cm}) \\
\text { [Range] }\end{array}$ & $\begin{array}{l}1.37^{*} \\
{[0.98-1.90]}\end{array}$ & $\begin{array}{l}1.50 * \\
{[0.72-1.82]}\end{array}$ & 0.64 \\
\hline $\begin{array}{l}\text { Minimum Distance }(\mathrm{cm}) \\
\text { [Range] }\end{array}$ & $\begin{array}{l}0.56 * * \\
{[0.0-1.14]}\end{array}$ & $\begin{array}{l}0.92^{* *} \\
{[0.17-1.44]}\end{array}$ & 0.1 \\
\hline $\begin{array}{l}\text { PTV Overlap With Rectum }\left(\mathrm{cm}^{3}\right) \\
\text { [Range] }\end{array}$ & $\begin{array}{l}0.05 \\
{[0.0-0.74]}\end{array}$ & $\begin{array}{l}0.0 \\
{[0.0-0.58]}\end{array}$ & 1 \\
\hline Property & Hydrogel & V-IRS & P-Value \\
\hline $\begin{array}{l}\text { Median Distance }(\mathrm{cm}) \\
\text { [Range] }\end{array}$ & $\begin{array}{l}0.93^{*} \\
{[0.48-1.57]}\end{array}$ & $\begin{array}{l}1.83^{*} \\
{[1.54-2.18]}\end{array}$ & 0.008 \\
\hline $\begin{array}{l}\text { Minimum Distance }(\mathrm{cm}) \\
\text { [Range] }\end{array}$ & $\begin{array}{l}0.31 * * \\
{[0.1-0.49]}\end{array}$ & $\begin{array}{l}0.81 * * \\
{[0.1-1.41]}\end{array}$ & 0.30 \\
\hline $\begin{array}{l}\text { PTV Overlap With Rectum }\left(\mathrm{cm}^{3}\right) \\
\text { [Range] }\end{array}$ & $\begin{array}{l}0.16 \\
{[0.0-3.25]}\end{array}$ & $\begin{array}{l}0.0 \\
{[0.0-1.58]}\end{array}$ & 1 \\
\hline
\end{tabular}

RBI: Rectum Balloon Implant, V-IRS: Virtual Implantable Rectum Spacer, PTV: Planning Target Volume

*the median of the median distance between the rectum and the prostate over all patients

** the median of the minimum distance between the rectum and the prostate over all patients

Supplementary Table S4.3: An overview of the values used in the Markov CE model for the cost-effectiveness of the IRS

\begin{tabular}{l|ll} 
Utilities & Value & SE \\
\hline Prostate cancer without treatment-related toxicity & 0.9 & 0.05 \\
Prostate cancer with severe late GI toxicity (grade>=2) & 0.727 & 0.04 \\
Costs & Value & $\mathrm{Cl}$ \\
Yearly costs prostate cancer without toxicity & $€ 323$ & $€ 215-€ 430$ \\
Yearly costs late grade 2 Gl toxicity & $€ 481$ & $€ 160-€ 482$ \\
Yearly costs late grade 3 Gl toxicity & $€ 4,051$ & $€ 1,876-€ 5,832$ \\
Proportion grade 2 (of all grade>=2) & 0.75 & $0.652-0.848$ \\
Cost IRS treatment & $€ 1,700$ & $€ 1,300-€ 2,100$ \\
\hline
\end{tabular}

IRS: implantable rectum spacer, GI: gastro-intestinal, SE: standard error, Cl: confidence interval 
Supplementary Table S4.4: An overview of the iNMB of the RBI and the hydrogel spacer after rescaling of the treatment plan to a target dose of $80 \mathrm{~Gy}$ in 32 fractions.

RBI

\begin{tabular}{r|ll}
\hline iNMB original plan & $-€ 1158$ & $-€ 1032$ \\
[range] & {$[-€ 2049-€ 481]$} & {$[-€ 1497-€ 328]$} \\
iNMB scaled plan & $-€ 442$ & $-€ 379$ \\
[range] & {$[-€ 1878-€ 3873]$} & {$[-€ 1476-€ 3631]$} \\
\hline \multicolumn{2}{r}{ Hydrogel Spacer } & $\boldsymbol{V}$-IRS \\
\hline iNMB original plan & $-€ 669$ & $-€ 567$ \\
[range] & {$[-€ 2308-€ 526]$} & {$[-€ 1525-€ 542]$} \\
iNMB scaled plan & $€ 376$ & $€ 619$ \\
[range] & {$[-€ 2769-€ 2057]$} & {$[-€ 1210-€ 2333]$} \\
\hline
\end{tabular}

RBI: Rectal Balloon implant, V-IRS: Virtual Implantable Rectum Spacer, iNMB: incremental Net Monetary Benefit. 


\section{Chapter 5}

\section{Development of an isotoxic Decision Support System integrating genetic markers of toxicity for the implantation of a rectum spacer}

Authors:

Yvonka van Wijk, Ben Vanneste, Arthur Jochems, Sean Walsh, Cary Oberije, Michael Pinkawa, Bram Ramaekers, Ana Vega, Philippe Lambin

Adapted from:

"Development of an isotoxic decision support system integrating genetic markers of toxicity for the implantation of a rectum spacer." Acta Oncologica 57.11 (2018): 1499-1505.

DOI: 10.1080/0284186X.2018.1484156 


\section{Abstract}

Introduction: Previous studies revealed that dose escalated radiotherapy for prostate cancer patients leads to higher tumour control probabilities (TCP) but also to higher rectal toxicities. An isotoxic model was developed to maximize the given dose while controlling the toxicity level. This was applied to analyse the effect of an implantable rectum spacer (IRS) and extended with a genetic test of normal tissue radio-sensitivity. A virtual IRS (V-IRS) was tested using this method. We hypothesized that the patients with increased risk of toxicity would benefit more from an IRS.

Materials and Methods: 16 localised prostate cancer patients implanted with an IRS were included in the study. Treatment planning was performed on Computed Tomography (CT) images before and after the placement of the IRS and with a V-IRS. The normal tissue complication probability (NTCP) was calculated using a QUANTEC reviewed model for Grade>=2 late rectal bleeding and the number of fractions of the plans were adjusted until the NTCP value was under $5 \%$. The resulting treatment plans were used to calculate the TCP before and after placement of an IRS. This was extended by adding the effect of two published genetic single nucleotide polymorphisms (SNPs) for late rectal bleeding.

Results: The median TCP resulting from the optimized plans in patients before the IRS was 75.1\% [32.6-90.5\%]. With IRS, the median TCP is significantly higher: 98.9\% [80.8-99.9\%] ( $P<0.01)$. The difference in TCP between the V-IRS and the real IRS was $1.8 \%$ [0.0-18.0\%]. Placing an IRS in the patients with SNPs improved the TCP from 49.0\% [16.1-80.8\%] and 48.9\% [16.0-72.8\%] to 96.3\% [67.0-99.5\%] and 90.1\% [49.0-99.5\%] $(\mathrm{P}<0.01)$ respectively for either SNP.

Conclusion: This study was a proof-of-concept for an isotoxic model with genetic biomarkers with a V-IRS as a multifactorial decision support system for the decision of a placement of an IRS. 


\section{Introduction}

Intensity modulated radiotherapy (IMRT) is one of the primary curative treatments for patients with prostate cancer. Studies have revealed that dose escalation leads to better biochemical progression free survival ${ }^{[1,2]}$. However, the proximity between the anterior rectal wall and the prostate allows for limited dose escalation without resulting in severe gastro-intestinal (GI) toxicity ${ }^{[3-5]}$.

The implantable rectum spacer (IRS) is a device developed to spare the rectum by increasing the distance between the anterior rectal wall and the prostate, thus reducing the overlap between the rectal wall and the high dose region ${ }^{[6-10]}$. Recent studies have shown that this device significantly reduces the dose in the rectum, thus reducing the risk of complications, for approximately $20 \%$ of the patients ${ }^{[5]}$. For these patients, the IRS would allow for same radiation dose and cure rate with decreased rectal toxicity rates. There is increasing evidence supporting the role of several genetic variants in the development of radiation-induced toxicity to distinguish the patients having increased risk factors for severe rectal and urinary toxicity ${ }^{[11,12]}$. We hypothesize that the patients with increased risk of radiationinduced rectal toxicity would benefit more from an IRS. Furthermore, the use of IRS in prostate cancer patients allows for more dose escalation, with consequently improving their prospective therapeutic ratio.

In this proof-of-concept study we developed a method to determine the amount of possible dose escalation with the resulting tumour control probability (TCP) without exceeding the set of boundaries for the complication risks: the socalled "isotoxic dose escalation" approach ${ }^{[13-15]}$. This method is applied to compare the possible dose escalation on patients with plans available before and after the placement of an IRS. This model is combined with a so-called 'virtual IRS' (V-IRS) [16]. This method uses image deformation to obtain CT images of patients with a predicted IRS position without having to place one (watch the animation: https:// www.youtube.com/watch?v=tDlagSXMKqw). Furthermore, this method helps identify the patients for whom significantly higher TCP is possible by placing an IRS.

To further individualize the predictions done for patients, the patients radiosensitivity needs to be taken into account. Studies have shown that the difference in radio-sensitivity between patients are likely due to common genetic variants, such as single nucleotide polymorphisms (SNPS). We applied the developed method to compare the results for patients using the model including and not including the genetic biomarker.

\section{Materials and methods}

\section{Patient characteristics}

For this study, 16 patients with localized prostate cancer who had signed an informed consent were included (see table in Supplementary Table S5.1). Two 
different commercial types of IRS's are used: 8 had received a rectum balloon implant (RBI) (BioProtect Ltd, Israel) ${ }^{[6]}$ and 8 had a hydrogel spacer (HGS) (SpaceOARTM System, Augmenix Inc., Waltham, MA) placed. The hydrogel and RBI patients included in this study were consecutively selected in 2011 and 2015, respectively. Both studies were approved by their local ethics committees ${ }^{[7]}$. The RBI patients were included in a clinical trial between 2015 and 2016 (registration number: 14-38-03/09-internal-6335 ${ }^{[10]}$. As this is a consecutive case study, it is assumed these patients are representative of average prostate cancer patients.

Imaging and treatment planning

Standard Computed tomography (CT) imaging was performed twice on all patients while they were in supine position: once before and once after placement of an IRS. These images had a slice thickness of $5 \mathrm{~mm}$ for the patients with a HGS, and $3 \mathrm{~mm}$ for those with a RBI. The pixel size was $1 \mathrm{~mm}$ for all images

The clinical target volume (CTV) was defined as the prostate for 8 patients, and the prostate including seminal vesicles for the rest and the planning target volume (PTV) was obtained by extending the CTV with $6 \mathrm{~mm}$ in all directions to account for uncertainties in planning an treatment delivery. The rectum volumes were delineated as a solid organ, including the rectum as well as the anal canal. The same experienced radiation oncologist (BV) did all delineations.

Plans were performed by a certified technician on all CT images, yielding two treatment plans for each patient (one without IRS, one with IRS). The treatment plans were designed for dose delivery with a knowledge-based volumetric modulated arc technique with $10 \mathrm{MV}$ photon beams (Eclipse Version ICD-10, Varian Medical Systems Inc., Palo Alto, USA). Dose delivery was planned to be done in 28 fractions of 2.5 Gray $(G y)^{[17]}$. Dose constraints held during treatment planning are shown in Supplementary Table S5.2.

\section{Isotoxic model}

\section{Dose calculations}

Treatment planning was performed in fractions of 2.5 Gy instead of $2 \mathrm{~Gy}$, which is the case for the treatment plans on which the NTCP model was based. For this reason, the equivalent 2 Gy dose (EQD2) in the rectum resulting from the treatment plan is calculated and used as input for the model. This is done using the Withers equation ${ }^{[18]}$ :

$$
E Q D 2=D \cdot \frac{d+^{\alpha} / \beta}{2+^{\alpha} / \beta}
$$


Here $D$ is the total dose, $d$ is the fraction dose and $\alpha / \beta$ represent the fractionation sensitivity of the organ. For late rectal toxicity, the $\alpha / \beta$ ratio has been estimated to be $3 \mathrm{~Gy}^{[19]}$.

\section{NTCP model}

The NTCP model used is the Lyman-Kutcher-Burman (LKB) mode ${ }^{[20]}$ that was reviewed by the QUANTEC group (quantitative analysis of normal tissue effects in the clinic). The LKB model is a dose response curve that uses the generalized equivalent uniform dose (gEUD) as a dose parameter, which is obtained using the DVH, and a parameter $n$, which determines which part of the DVH is statistically most important. The curve is dependent on the dose required for an NTCP of $50 \%$ (D50) and the steepness of the curve (m). The QUANTEC-recommended parameters are TD50 $=76.9, \mathrm{n}=0.09$ and $\mathrm{m}=0.13$ for grade $>=2$ late rectal toxicity.

The NTCP model used is a Lyman-Kutcher-Burman (LKB) model that was reviewed by the QUANTEC group (quantitative analysis of normal tissue effects in the clinic). This model is described by the equations (5.2-5.4) shown below:

$$
\begin{gathered}
N T C P=\frac{1}{\sqrt{2 \pi}} \int_{-\infty}^{t} e^{-\frac{x^{2}}{2}} d x \\
t=\frac{g E U D-T D 50}{m \cdot T D 50} \\
g E U D=\left(\sum_{i} v_{i} d_{i}^{1 / n}\right)^{n}
\end{gathered}
$$

where gEUD stands for generalized equivalent uniform dose, $i$ is the number of the DVH bin, $v_{i}$ is the volume of the bin and $d_{i}$ is the dose. The QUANTEC-recommended parameters are TD50 $=76.9, \mathrm{n}=0.09$ and $\mathrm{m}=0.13$ for grade $>=2$ late rectal toxicity.

\section{Single Nucleotide Polymorphisms}

This NTCP model was expanded as a proof-of-concept with genetic markers of rectal toxicity, for which we used published results of a meta-analysis of GWASs (Genome Wide Association Study) for genetic markers of late toxicity, in prostate cancer patients treated with radiotherapy ${ }^{[12]}$. The two SNPs most associated with rectal bleeding, that approach meta-analysis significance, were selected for the initial proof-of-concept: rs141044160 (SNP 1) with an odds ratio of 2.68 (P $=2.26^{*} 10^{-6}$ ) and rs7432328 (SNP 2), with an odds ratio of $3.36\left(P=3.32 * 10^{-6}\right)$. The SNPs were incorporated into the dose response curve using a published mathematical method ${ }^{[21]}$. In this method, clinical parameters are incorporated into a dose response by separating the curve for the overall population into two curves: one for the population with the SNP risk allele and one for the population without. 
This is done using the odds ratio and the proportion between the population with and without the risk factor. The proportion of the population with the SNP risk allele can be calculated using the minor allele frequency (MAF). For this study, we demonstrated the effects of the SNPS on the NTCP model by applying it on the patient cohort, assuming they were carriers for either SNP risk allele.

In order to incorporate a SNP into the LKB model, the dose response curve was split into two different dose response curves: one for the population with the SNP and one without. The dosimetric factor used by the LKB is the generalized equivalent univorm dose (gEUD), and the parameters which determine the shape of the dose response curve are the TD50, which is the gEUD needed for a $50 \%$ response, and $\mathrm{m}$, which is inversely proportional to the gradient at TD50. In order to split the LKB curve into two, we need to determine the new TD50 and the new $m$ for both curves. For this, we need to know the relationship of the response of the curve without SNP and with SNP, $\mathrm{p}_{0}$ and $\mathrm{p}_{1}$ respectively, to the original curve $\mathrm{p}$.

The prevalence factor of the SNP can be calculated using the minor allele frequency as shown in equation (5.5):

$$
s=2 \cdot M A F+M A F^{2} \approx 2 \cdot M A F
$$

Where $\mathrm{s}$ is the prevalence. Because the total probability of toxicity needs to remain the same over the whole populations, the relationship of the two new curves to the original can be described as shown in equation (5.6):

$$
p=s \cdot p_{1}+(1-s) p_{0}
$$

The odds ratio (OR) can be described as function of $p_{0}$ and $p_{1}$ as shown in equation (5.7):

$$
O R=\frac{p_{1}}{1-p_{1}} / \frac{p_{0}}{1-p_{0}}
$$

Which allows us to describe equation (5.6) as shown in equation (5.8):

$$
p=s \frac{O R \cdot p_{0}}{p_{0}(O R-1)+1}+(1-s) p_{0}
$$

Since the OR of each SNP is reported, as well as the MAF, we can now use the method reported in Appelt\&Vogelius (2012) to find the correct values for TD50 and $m$ corresponding to the low and high risk curves.

\section{TCP model}

The used TCP mode ${ }^{[22]}$ was built to predict the clinical response to external beam radiotherapy in patients with low, intermediate and high risk prostate cancer, and is available on http://predictcancer.org/. This model utilizes realistic radio biologically 
input parameters and works for a wide range of treatment strategies ${ }^{[23]}$.

The model is described using equation (5.9):

$$
T C P=\left(\frac{1}{k}\right) \sum_{i=1}^{k}\left(\exp \left(-N_{0} S(D)_{i}\right)\right)
$$

where $k$ represents groups of patients with separate radiosensitivity, $N_{0}$ is the initial clonogen sum and $S(D)$ is the surviving fraction of a population of cells irradiated by a total uniform dose $D$.

The inputs to the TCP model are the number of fractions, the fraction dose, the treatment modality and the risk group of the patient. The prostate-specific antigen (PSA), TNM and Gleason score are the input factors to the classification of the risk group: low, medium or high ${ }^{[24]}$. To evaluate the result of dose escalation, only the fraction dose is varied in this study to test the amount of dose escalation possible without exceeding NTCP limits.

\section{Isotoxic method}

The proposed model uses an optimized treatment plan for a patient, calculates the DVH in the organs at risk and scales this DVH by changing the fraction dose used during treatment planning. The fraction dose is varied in order to obtain a maximum TCP value without exceeding the cut-off value set for the NTCP value, which, for this paper, is arbitrarily chosen to be $5 \%$ (Figure 5.1). The fraction dose for a plan with 28 fractions advised by the model can vary between 1.5 and 3.5 Gy. These limits are chosen because a plan with 28 fractions with less than 1.5 Gy, resulting TCP was modelled to always be lower than $25 \%$, and more than 3.5 fractions was to always result in a TCP higher than 95\%. In reality 1.5 Gy in 28 fractions would not be for curative treatment, but in this study these plans are calculated for theoretical comparison.

The method only takes into account rectal toxicity, but as the bladder is also an organ at risk, we added a constraint to the bladder dose ${ }^{[25]}$, as is shown in Supplementary Figure S5.1.

To use the method to select patients for whom the placement of an IRS is beneficial, a minimum increase in TCP should be chosen. For this study, two cutoff values were used for the increase in TCP as a result of the placement of an IRS: $25 \%$ and $50 \%$. 


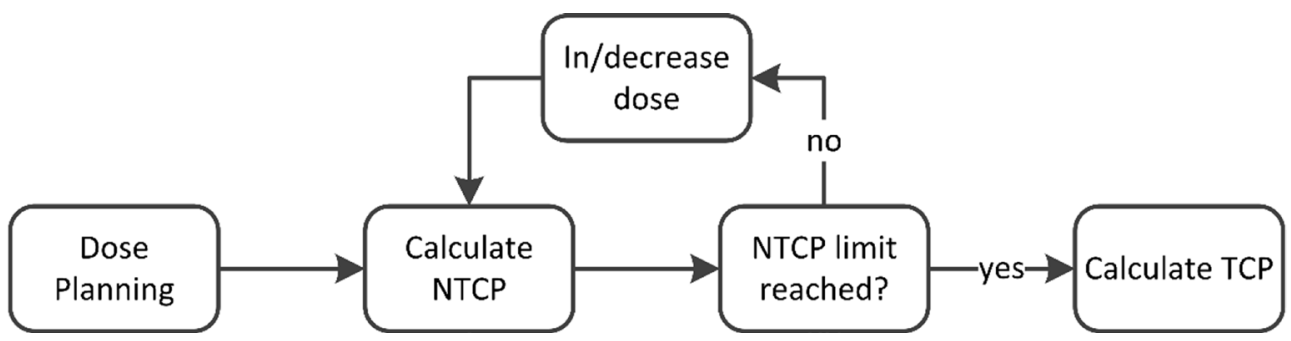

Figure 5.1. A flowchart of the isotoxic model. The model loads a treatment plan made by an expert and increases or decreases the fraction dose. Each time fraction dose is increased or decreased, the normal tissue complication probability (NTCP) is recalculated until a set cutoff value is reached. The tumour control probability (TCP) is hen calculated

\section{Virtual Spacer}

In order to use the isotoxic model to predict the improvement of the IRS in a specified patient, CT images of a patient are needed before the placement of the IRS. To this end, a previously developed V-IRS was used ${ }^{[16]}$. This V-IRS uses a model IRS derived from 7 delineations of a RBI and uses image deformation to insert the model into a CT image of a patient without an IRS, thus creating a virtual image of the patient with an IRS. This virtual image is then subjected to treatment planning, and the isotoxic model is used to calculate the highest possible TCP without exceeding the NTCP limit. In this study we test the V-IRS by comparing the results of the isotoxic model to those of the actual IRS.

\section{Statistics}

We used two-tailed Wilcoxon signed rank tests to determine whether the increase in optimal TCP for the plans without IRS and with IRS was significant. The test was also applied to test if the difference between the NTCP values before and after placement was significant. P-values of less than 0.05 were considered significant.

\section{Results}

\section{Dose escalation}

Giving more dose per fraction increases both the TCP and the NTCP predictions for a given patient (Figure 5.2). The original plans (before applying the isotoxic method) consisted of 28 fractions of $2.5 \mathrm{~Gy}$, which, for this set of patients corresponds to a median TCP of $80.8 \%$ with a range of [51.8-92.0\%]. The median fraction dose allowed by the model without exceeding the NTCP constraint before the placement of an IRS was 2.5 Gy [2.4-2.7 Gy] (Table 5.1), and corresponds 
to $75.1 \%$ [32.6-90.5\%] TCP. The TCP for the patients when given a conformal treatment plan of $2 \mathrm{~Gy}$ in 35 fractions corresponds to a TCP of $60.8 \%$ [26.3-80.9\%]. Figure 2 shows that to obtain a median TCP of at least $90 \%$, the dose needs to be escalated further to 2.7 Gy per fraction for a treatment plan with 28 fractions, which was possible for 2 of the 16 patients while holding to the pre-set accepted toxicity rates. For 5 of the patients 2.6 Gy per fraction could be given, and for the remaining patients, no dose escalation was possible. 
Table 5.1 - Overview Diagnosis support systems

\begin{tabular}{l|lll} 
& $\begin{array}{l}\text { Before IRS } \\
\text { Median [range] }\end{array}$ & $\begin{array}{l}\text { After IRS } \\
\text { Median [range] }\end{array}$ & $\begin{array}{l}\text { V-IRS } \\
\text { Median [range] }\end{array}$ \\
\hline EUD (Gy) & 58.5 & 59.0 & 59.0 \\
& {$[57.2-60.4]$} & {$[57.6-60.3]$} & {$[57.5-60.2]$} \\
Fraction (Gy) & 2.5 & 2.8 & 3.1 \\
& {$[2.4-2.7]$} & {$[2.5-3.4]$} & {$[2.5-3.9]$} \\
NTCP late RB (\%) & 3.5 & 3.7 & 3.6 \\
& {$[2.5-5.2]$} & {$[2.7-5.1]$} & {$[2.6-4.8]$} \\
TCP (\%) & 75.1 & 98.9 & 99.3 \\
& {$[32.6-90.5]$} & {$[80.8-99.9]$} & {$[80.8-100.0]$} \\
\hline
\end{tabular}

IRS: Implantable Rectum Spacer, V-IRS: virtual IRS, EUD: equivalent uniform dose in rectum with $n=0.09$, TCP: Tumor Control Probability, NTCP: Normal Tissue Complication Probability, RB: rectal bleeding

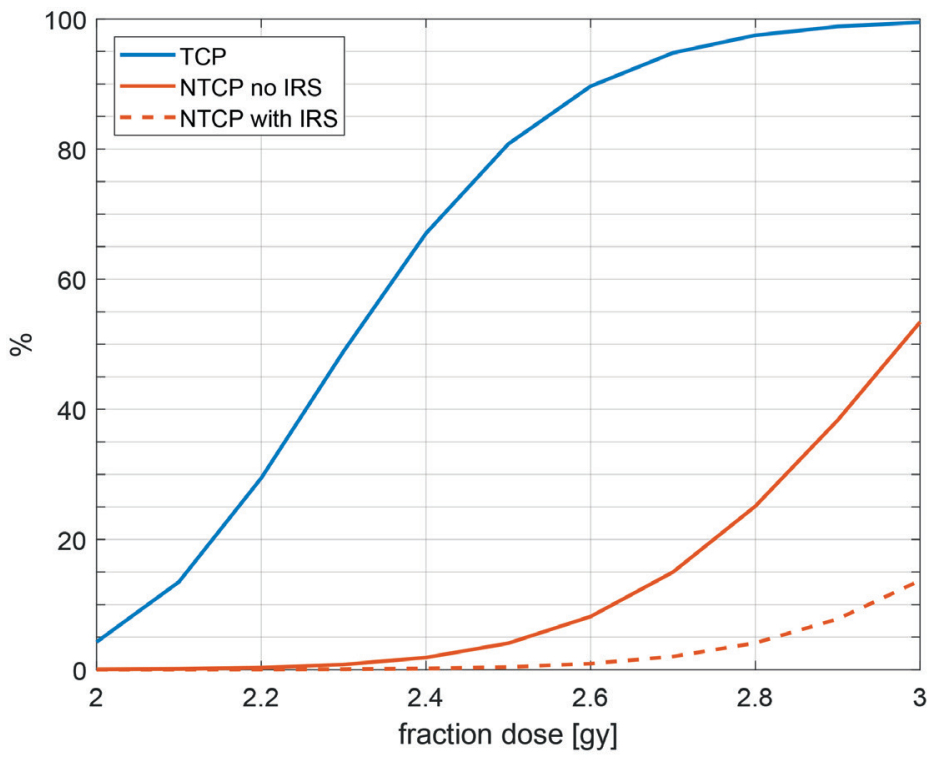

Figure 5.2. The median tumour control probability (TCP) and the median normal tissue complication probability (NTCP) is plotted against the fraction dose for the patients without implantable rectum spacer (IRS) and with IRS.

\section{IRS gain}

The placement of an IRS resulted in an increase in advised fraction dose of 0.4 Gy [-0.1-0.9 Gy] on average for all patients. Note that the minimum increase is negative, which means this is a decrease. This results in an absolute increase in TCP of $21.1 \%$ [-8.9-57.9\%] which is statistically significant $(P<0.01)$ (Figure 5.3). Patient specific outcomes have been plotted in a similar manner in Supplementary Figure 
S5.2. In only one patient the TCP after the placement of an IRS is lower than before placement. For this patient, the rectum volume had decreased after the placement of an IRS (from $268.9 \mathrm{~cm}^{3}$ to $156.1 \mathrm{~cm}^{3}$ ).

If an increase in TCP of $25 \%$ is taken as a threshold for the decision to place an IRS, then 7 of the 16 patients would be selected by the model for the placement of an IRS. Two patients even had an increased TCP of $50 \%$ resulting from the IRS.

The difference in the NTCP resulting from the optimized plan for the patients without IRS and with IRS is $0.15 \%$ and is not significant $(P=0.47)$. We performed an analyses for different NTCP limits in Supplementary Figure S5.3.

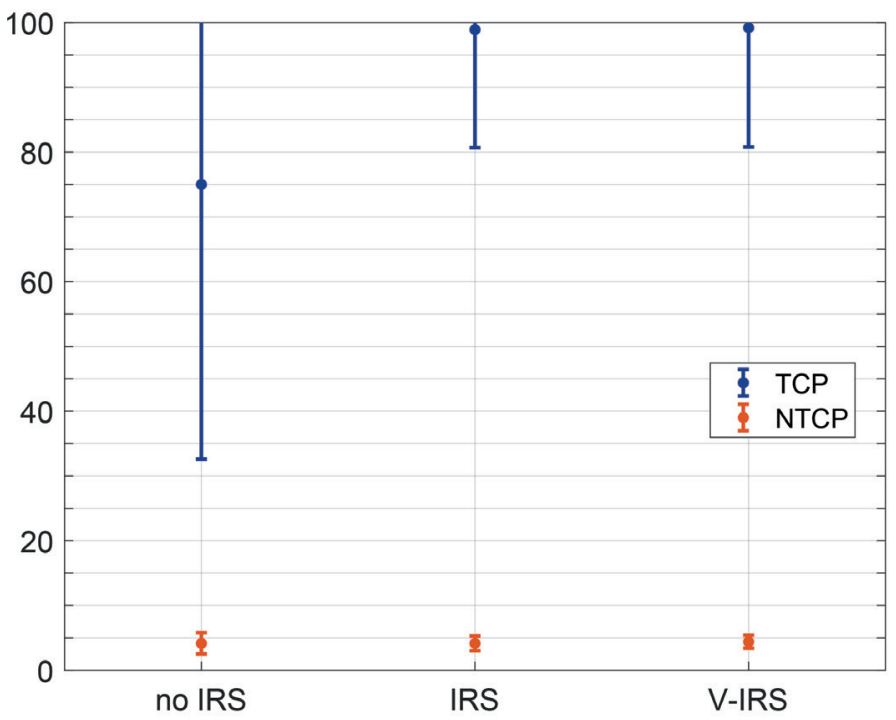

Figure 5.3. The median tumour control probability (TCP) and range, as well as the median normal tissue complication probability (NTCP) and range, for the patients without implantable rectum spacer (IRS), with IRS and with the virtual IRS (V-IRS) is shown.

\section{Performance V-IRS}

The difference in fractionation result between the V-IRS and the real IRS was a median of $0.3 \mathrm{~Gy}[0-0.8 \mathrm{~Gy}]$, which translates to a median TCP difference of $1.8 \%$ [0.0-18.0\%]. The difference between the optimized TCP for the real IRS and the V-IRS was not significant $(P=0.2)$. There were two patients for whom the difference in TCP between the V-IRS plan and the IRS plan was 18\%, and in both patients the rectum volume had changed significantly $(57.9 \%$ and $58.1 \%$ volume decrease in the image after the IRS).

When considering a minimum increase in TCP of $25 \%$ for the placement of an IRS, then 6 of the 16 patients would be selected by the model using the V-IRS, in contrary to the 7 selected using the actual IRS. The increase predicted by the V-IRS 
was underestimated compared to the real IRS in one patient, with an increase of $13.7 \%$ versus $27.7 \%$. The two patients who had an increased TCP of $50 \%$ resulting from the real IRS, had the same increase predicted by the V-IRS.

\section{SNP results}

Figure 5.4 shows the TCP and the NTCP as function of the fraction dose, and it can be observed that though the TCP is the same as for the patients without SNPS, the NTCP curve is much steeper for both SNPs.

The median NTCP for the original plan (28 fractions of $2.5 \mathrm{~Gy}$ ) was $11.2 \%$ [2.219.6\%] and 13.1\% [2.7-22.5\%] for SNP 1 and SNP 2 respectively, which is reduced significantly $(P<0.01)$ when using the isotoxic method. The results show (Table 5.2) that if the patients are carriers of the chosen SNPS, the TCP while restraining the NTCP would be $49.0 \%$ for SNP 1, and $48.9 \%$ for SNP 2. The placement of an IRS resulted in significantly better TCP values $(P<0.01)$, with a median of $96.3 \%$ [67.0-99.5\%] and 90.1\% [49.0-99.5\%] for SNP 1 and SNP 2 respectively, which was significantly higher than without IRS $(P<0.01)$ for both SNPS. Four other SNPs were found to be near significant for late rectal bleeding; results for these SNPs are shown in Supplementary Figure S5.4. We performed a sensitivity analyses on a range of odds ratios to show the possible effects of different SNPs for late rectal bleeding in Supplementary Figure S5.5.
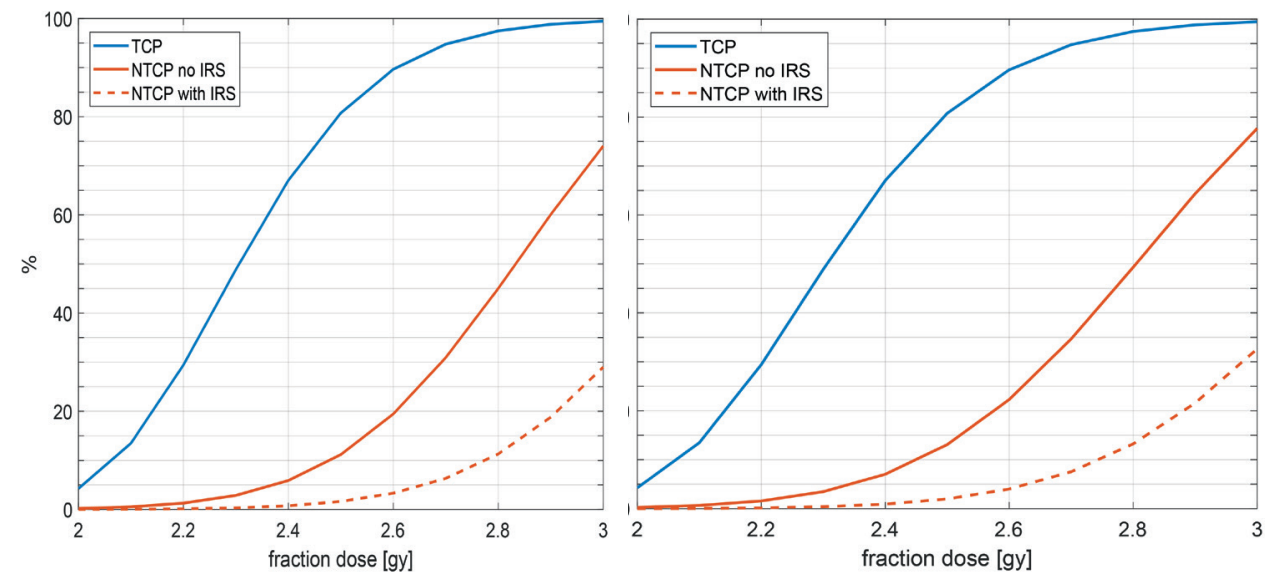

Figure 5.4. The median tumour control probability (TCP) and the median normal tissue complication probability (NTCP) is plotted against the fraction dose for the patients with SNPS (a: rs141044160 and b: rs7432328) without implantable rectum spacer (IRS) and with IRS. 
Table 5.2 - Overview Diagnosis support systems

\begin{tabular}{|c|c|c|c|}
\hline SNP 1 & $\begin{array}{l}\text { Before IRS Median } \\
\text { [range] }\end{array}$ & After IRS Median [range] & $\begin{array}{l}\text { V-IRS Median } \\
\text { [range] }\end{array}$ \\
\hline EUD (Gy) & $\begin{array}{l}53.9 \\
{[52.1-55.1]}\end{array}$ & $\begin{array}{l}54.1 \\
{[52.7-55.2]}\end{array}$ & $\begin{array}{l}53.9 \\
{[53.0-54.9]}\end{array}$ \\
\hline Fraction (Gy) & $\begin{array}{l}2.4 \\
{[2.2-2.6]}\end{array}$ & $\begin{array}{l}2.7 \\
{[2.4-3.2]}\end{array}$ & $\begin{array}{l}2.9 \\
{[2.3-3.7]}\end{array}$ \\
\hline NTCP late RB (\%) & $\begin{array}{l}3.8 \\
{[2.5-4.9]}\end{array}$ & $\begin{array}{l}3.9 \\
{[2.8-4.9]}\end{array}$ & $\begin{array}{l}3.8 \\
{[3.0-4.6]}\end{array}$ \\
\hline TCP (\%) & $\begin{array}{l}49.0 \\
{[16.1-80.8]}\end{array}$ & $\begin{array}{l}96.3 \\
{[67.0-99.5]}\end{array}$ & $\begin{array}{l}96.4 \\
{[48.9-100.0]}\end{array}$ \\
\hline SNP 2 & $\begin{array}{l}\text { Before IRS } \\
\text { Median [range] }\end{array}$ & $\begin{array}{l}\text { After IRS } \\
\text { Median [range] }\end{array}$ & $\begin{array}{l}\text { V-IRS } \\
\text { Median [range] }\end{array}$ \\
\hline EUD (Gy) & $\begin{array}{l}53.2 \\
{[51.0-54.4]}\end{array}$ & $\begin{array}{l}52.9 \\
{[51.5-54.1]}\end{array}$ & $\begin{array}{l}53.3 \\
{[51.3-54.3]}\end{array}$ \\
\hline Fraction (Gy) & $\begin{array}{l}2.3 \\
{[2.2-2.5]}\end{array}$ & $\begin{array}{l}2.6 \\
{[2.3-3.2]}\end{array}$ & $\begin{array}{l}2.9[ \\
2.3-3.7]\end{array}$ \\
\hline NTCP late RB (\%) & $\begin{array}{l}3.8 \\
{[2.3-5.0]}\end{array}$ & $\begin{array}{l}3.8 \\
{[2.6-5.2]}\end{array}$ & $\begin{array}{l}4.1 \\
{[2.5-5.2]}\end{array}$ \\
\hline TCP (\%) & $\begin{array}{l}48.9 \\
{[16.0-72.8]}\end{array}$ & $\begin{array}{l}90.1 \\
{[49.0-99.5]}\end{array}$ & $\begin{array}{l}96.4 \\
{[49.0-100.0]}\end{array}$ \\
\hline
\end{tabular}

IRS: Implantable Rectum Spacer, V-IRS: virtual IRS, EUD: equivalent uniform dose in rectum with $n=0.09$, TCP: Tumor Control Probability, NTCP: Normal Tissue Complication Probability, SNP: SingleNucleotide Polymorphism, SNP 1: rs141044160, SNP 2: rs7432328

\section{Discussion}

In this study, we have developed an isotoxic method integrating genetic markers of rectal radio-sensitivity combined with a V-IRS which can be used to support the decision for the implantation of an IRS for a specified patient or not. The method applies image deformation to a CT image of a patient without IRS, and creates a virtual CT image with IRS. The method calculates the maximum dose per fraction that can be given without exceeding an upfront determined limit for the NTCP value for late rectal bleeding.

The results show that a higher prescribed dose should be given to improve TCP, and can be safely administered when combined with an IRS. This method can be used as the basis of a multifactorial DSS ${ }^{[26]}$, which can be used as a meta-treatment planning system ${ }^{[27]}$ to decide in which patients to place an IRS, as well as advise on how much dose escalation is possible to apply for a specified patient ${ }^{[28,29]}$. This method also allows for shared decision making, taking into account the patients priorities, such as cure rate or risk of toxicity.

Our method has two main strengths. Firstly, the method uses NTCP prediction values to predict the amount of dose escalation possible while keeping rectal 
toxicities under control. Applying this method in the clinic would allow dose escalation to be used more safely and improve outcomes for patients ${ }^{[30]}$. Secondly, treatment planning with an IRS can be applied to a CT of a patient without the real implantation of the IRS itself. This means that the increase in TCP with the IRS for the specified patient can be estimated without having to put the patient through the discomfort of the implantation, and without the additional costs of the placement of the IRS, before it has been established that an IRS will be beneficial.

The results showed that the system would advise further dose escalation even without the placement of an IRS, which would result in better outcomes for these patients. The system foresees a gain in TCP for 7 of the 16 patients, showing the model can be used as to identify patients for whom an IRS is beneficial. Using the V-IRS, 6 of the 7 patients for whom the spacer would be significantly effective could be identified. The patient not identified had very different rectal fillings before and after IRS placement. This demonstrates that this DSS has the potential to identify patients who benefit most from an IRS. This improves the cost-effectiveness of the IRS by only placing it in patients who would benefit from it, while also improving their survival and quality of life.

During this study, SNPs were incorporated into the NTCP model and tested on the patient cohort, assuming all were heterogeneous for either SNP. Because the toxicity risk of a carrier is larger than for a non-carrier, these patients could normally be considered for alternative treatments, such as surgery. However, the method presented here has the potential to identify patients with this SNP who could be treated safely with radiotherapy after placement of an IRS. This would be beneficial, since placing an IRS is less invasive and has a very low to no impact on the quality of life, especially in comparison with surgery ${ }^{[8]}$. This would also be beneficial for patients who don't want an operation or who are not eligible for surgery.

One of the main limitations of the study was the small amount of patients included. Though they were sufficient for a first proof-of-concept, a more elaborate study including a larger amount of patients is required for internal validation of the method, and additional data sets are required for external validation.

Furthermore, the LKB model uses the entire DVH as a predictor for late rectal bleeding, which is partly effected by the rectal volume, a factor which varies strongly over time ${ }^{[31]}$. The model cannot predict these changes in rectal volume, and for some patients this may influence the outcome. For two patients, this difference in volume was as much as $150 \mathrm{~cm}^{3}$, and for one patient this resulted in a large overestimation of the NTCP by the V-IRS. For another patient the decrease in rectal volume even resulted in an increased NTCP after the placement of the IRS. A possible solution to this problem is to submit patients with very full rectums to rescanning, or for patients to be given a laxative prior to scanning. For future validation of the V-IRS, only CT scans with similar rectal filling before and after IRS placement should be 
used, as only then a fair comparison can be made. Finally, concerning optimising the treatment planning technique: it is possible to reduce intermediate dose levels (30-50 Gy) in the rectum region by an arc-therapy with an avoidance-region near the rectum.

Another possible error in both the TCP and the NTCP model is the $\alpha / \beta$ ratio, which varies between prostate, rectum and bladder. In order to fully understand the effect possible errors in the $\alpha / \beta$ ratio have on the calculated outcome, a sensitivity analyses should be done. However, considering the uncertainty in estimates for $\alpha / \beta$ ratio's results for these kind of analysis should always be interpreted carefully.

The V-IRS used in this study was based on a RBI, a closed system which has a predictable shape, while 8 of the patients were implanted with a HGS, which has a highly variable shape ${ }^{[32]}$. However, patients with a large increase in TCP resulting from a hydrogel spacer can still be identified using the V-IRS, and therefore it still has potential as the basis of the DSS, even for the hydrogel spacer. The V-IRS has been published in a proof-of-concept study but requires validation. The V-IRS performance is promising, however after validation it is expected to improve and consequently leading to better outcome results.

The integration of the SNPs is theoretical in this study, as none of the patients were sequenced and tested for the SNPs, and as the MAF is low (0.05 and 0.06), it is not likely any of them would be carriers. An improved method would be to build an NTCP model on a large patient cohort for whom sequencing has been done and for whom outcome data has been collected. Also, though SNPs have been identified that are significant for rectal toxicity in general, currently no SNPs have been found to be significant for late rectal bleeding specifically ${ }^{[12]}$. To date, six SNPS have been associated with rectal bleeding (11), of which two were used in this study. These SNPs approaching statistical significance in a meta-analysis ( $P<5 * 10^{-}$ $\left.{ }^{6}\right)$. Further validation in larger studies are needed before incorporate these SNPs in NTCP models. Once significant SNPs are identified, these can be incorporated into NTCP models.

The NTCP model is dependent on the DVH in the rectum; however, during treatment this DVH might vary depending on day-to-day variability of rectum filling. This could influence the accuracy of the NTCP model. In order to fully demonstrate the effect this has on the NTCP prediction, the real administered rectal dose should be computed. Also, the NTCP model used in this study only uses the DVH as a predictor for late rectal bleeding, however, studies have shown that several clinical parameters (diabetes, abdominal surgery, etc.) are also strong predictors. In future work, we intend to include NTCP models using clinical predictors into the model as well as validated SNPS. We also want to validate the method of combining published SNPS with NTCP models on a dataset for which sequencing was done, and for which outcomes were known. 
Including different endpoints for the toxicity models, such as incontinence, erectile dysfunction and urinary toxicity would serve to further improve patient specific decision making. Another good expansion of the model would be to add a cost-effectiveness analyses, which would enhance the cost-effectiveness of the IRS, a topic that is becoming increasingly important due to ever-expanding expenses in health care ${ }^{[33,34]}$. Another development would be to focus on hypofractionation as well. Possibilities for extreme hypofractionation, such as stereotactic radiotherapy, single fraction high dose rate brachytherapy or single shot proton therapy could be explored. This would serve to further increase patient prospects as well as costeffectiveness.

Before application in the clinic is possible, the DSS model needs to be further developed while taking into accounts the limits of treatment planning and application. When the model advices lower dose prescription than the original treatment plan, the V-MAT system might not be able to calculate the treatment plan. Analyses should be done on how easily prescription dose can be adjusted, and whether variation in fraction dose and/or number of fractions would be most effective.

The isotoxic model integrating genetic markers for rectal toxicity developed in this study can be used to evaluate a treatment plan, and test how much dose can be given without causing excessive damage to the rectum. Thus sparing the organs at risk at a chosen described level while optimizing the TCP. In combination with the V-IRS, this method can serve as the basis for a DSS for the implantation of an IRS.

\section{Disclosures of potential conflicts of interest}

Sean Walsh is a salaried employee of ptTheragnostic B.V. and CSO of OncoRadiomics SPRL. Philippe Lambin is co-inventors of IP related to Decision Support Systems and he is member of the advisory board of ptTheragnostic/DNAmito. Cary Oberije is employee and CEO of ptTheragnostic B.V.

\section{Acknowledgements}

Authors acknowledge financial support from ERC advanced grant (ERC-ADG-2015, $n^{\circ}$ 694812- Hypoximmuno). This research is also supported by the Dutch technology Foundation STW (grant $n^{\circ} 10696$ DuCAT \& n P14-19 Radiomics STRaTegy), which is the applied science division of NWO, and the Technology Programme of the Ministry of Economic Affairs. Authors also acknowledge financial support from the EU 7th framework program (REQUITE - $n^{\circ}$ 601826), Interreg V-A Euregio MeuseRhine ("Euradiomics"), Kankeronderzoekfonds Limburg from the Health Foundation Limburg and the Dutch Cancer Society. 


\section{References}

1. Kalbasi, A., J. Li, A. Berman, et al., Dose-Escalated Irradiation and Overall Survival in Men With Nonmetastatic Prostate Cancer. JAMA Oncol, 2015. 1(7): p. 897-906.

2. Zelefsky, M.J., X. Pei, J.F. Chou, et al., Dose escalation for prostate cancer radiotherapy: predictors of long-term biochemical tumor control and distant metastases-free survival outcomes. Eur Urol, 2011. 60(6): p. 1133-9.

3. Glass, A.S., J.E. Cowan, M.J. Fuldeore, et al., Patient demographics, quality of life, and disease features of men with newly diagnosed prostate cancer: trends in the PSA era. Urology, 2013. 82(1): p. 60-5.

4. Vanneste, B.G., L. Van De Voorde, R.J. de Ridder, et al., Chronic radiation proctitis: tricks to prevent and treat. Int J Colorectal Dis, 2015. 30(10): p. 1293-303.

5. Vanneste, B.G., A.L. Hoffmann, E.N. van Lin, et al., Who will benefit most from hydrogel rectum spacer implantation in prostate cancer radiotherapy? A model-based approach for patient selection. Radiother Oncol, 2016. 121(1): p. 118-123.

6. Gez, E., S. Cytron, R. Ben Yosef, et al., Application of an interstitial and biodegradable balloon system for prostate-rectum separation during prostate cancer radiotherapy: a prospective multi-center study. Radiat Oncol, 2013. 8: p. 96.

7. Pinkawa, M., N.E. Corral, M. Caffaro, et al., Application of a spacer gel to optimize three-dimensional conformal and intensity modulated radiotherapy for prostate cancer. Radiother Oncol, 2011. 100(3): p. 436-41.

8. Vanneste, B.G., K. van De Beek, L. Lutgens, et al., Implantation of a biodegradable rectum balloon implant: tips, Tricks and Pitfalls. Int Braz J Urol, 2017. 43.

9. Vanneste, B.G.L., Y. van Wijk, L.C. Lutgens, et al., Dynamics of rectal balloon implant shrinkage in prostate VMAT : Influence on anorectal dose and late rectal complication risk. Strahlenther Onkol, 2017.

10. Vanneste, B.G.L., Y. van Wijk, L.C. Lutgens, et al., Dynamics of rectal balloon implant shrinkage in prostate VMAT : Influence on anorectal dose and late rectal complication risk. Strahlenther Onkol, 2018. 194(1): p. 31-40.

11. Fachal, L., A. Gomez-Caamano, G.C. Barnett, et al., A three-stage genome-wide association study identifies a susceptibility locus for late radiotherapy toxicity at 2q24.1. Nat Genet, 2014. 46(8): p. 891-4.

12. Kerns, S.L., L. Dorling, L. Fachal, et al., Meta-analysis of Genome Wide Association Studies Identifies Genetic Markers of Late Toxicity Following Radiotherapy for Prostate Cancer. EBioMedicine, 2016. 10: p. 150-63.

13. Zindler, J.D., C.R. Thomas, Jr., S.M. Hahn, et al., Increasing the Therapeutic Ratio of Stereotactic Ablative Radiotherapy by Individualized Isotoxic Dose Prescription. J Natl Cancer Inst, 2016. 108(2).

14. De Ruysscher, D., A. van Baardwijk, J. Steevens, et al., Individualised isotoxic accelerated radiotherapy and chemotherapy are associated with improved long-term survival of patients with stage III NSCLC: a prospective population-based study. Radiother Oncol, 2012. 102(2): p. 228-33.

15. Bongers, M.L., D. de Ruysscher, C. Oberije, et al., Model-Based Cost-Effectiveness of Conventional and Innovative Chemo-Radiation in Lung Cancer. Int J Technol Assess Health Care, 2017. 33(6): p. 681-690. 
16. van Wijk, Y., B.G.L. Vanneste, S. Walsh, et al., Development of a virtual spacer to support the decision for the placement of an implantable rectum spacer for prostate cancer radiotherapy: Comparison of dose, toxicity and cost-effectiveness. Radiother Oncol, 2017. 125(1): p. 107-112.

17. Kupelian, P.A., T.R. Willoughby, C.A. Reddy, et al., Hypofractionated intensity-modulated radiotherapy (70 Gy at 2.5 Gy per fraction) for localized prostate cancer: Cleveland Clinic experience. Int J Radiat Oncol Biol Phys, 2007. 68(5): p. 1424-30.

18. Withers, H.R., H.D. Thames, Jr., and L.J. Peters, A new isoeffect curve for change in dose per fraction. Radiother Oncol, 1983. 1(2): p. 187-91.

19. Marzi, S., B. Saracino, M.G. Petrongari, et al., Modeling of alpha/beta for late rectal toxicity from a randomized phase II study: conventional versus hypofractionated scheme for localized prostate cancer. J Exp Clin Cancer Res, 2009. 28: p. 117.

20. Michalski, J.M., H. Gay, A. Jackson, et al., Radiation dose-volume effects in radiation-induced rectal injury. Int J Radiat Oncol Biol Phys, 2010. 76(3 Suppl): p. S123-9.

21. Appelt, A.L. and I.R. Vogelius, A method to adjust radiation dose-response relationships for clinical risk factors. Radiother Oncol, 2012. 102(3): p. 352-4.

22. Walsh, S. and W. van der Putten, A TCP model for external beam treatment of intermediate-risk prostate cancer. Med Phys, 2013. 40(3): p. 031709.

23. Stewart, R.D. and X.A. Li, BGRT: biologically guided radiation therapy-the future is fast approaching! Med Phys, 2007. 34(10): p. 3739-51.

24. D’Amico, A.V., R. Whittington, S.B. Malkowicz, et al., Biochemical outcome after radical prostatectomy, external beam radiation therapy, or interstitial radiation therapy for clinically localized prostate cancer. JAMA, 1998. 280(11): p. 969-74.

25. Viswanathan, A.N., E.D. Yorke, L.B. Marks, et al., Radiation dose-volume effects of the urinary bladder. Int J Radiat Oncol Biol Phys, 2010. 76(3 Suppl): p. S116-22.

26. Lambin, P., R.G. van Stiphout, M.H. Starmans, et al., Predicting outcomes in radiation oncology-multifactorial decision support systems. Nat Rev Clin Oncol, 2013. 10(1): p. 27-40.

27. Chetty, I.J., M.K. Martel, D.A. Jaffray, et al., Technology for Innovation in Radiation Oncology. Int J Radiat Oncol Biol Phys, 2015. 93(3): p. 485-92.

28. Lambin, P., J. Zindler, B. Vanneste, et al., Modern clinical research: How rapid learning health care and cohort multiple randomised clinical trials complement traditional evidence based medicine. Acta Oncol, 2015. 54(9): p. 1289-300.

29. Lambin, P., E. Roelofs, B. Reymen, et al., 'Rapid Learning health care in oncology' - an approach towards decision support systems enabling customised radiotherapy'. Radiother Oncol, 2013. 109(1): p. 159-64.

30. Reymen, B., A. van Baardwijk, R. Wanders, et al., Long-term survival of stage T4NO-1 and single station IIIA-N2 NSCLC patients treated with definitive chemo-radiotherapy using individualised isotoxic accelerated radiotherapy (INDAR). Radiother Oncol, 2014. 110(3): p. 482-7.

31. Hoogeman, M.S., M. van Herk, J. de Bois, et al., Strategies to reduce the systematic error due to tumor and rectum motion in radiotherapy of prostate cancer. Radiother Oncol, 2005. 74(2): p. 177-85.

32. Pinkawa, M., J. Klotz, V. Djukic, et al., Learning curve in the application of a hydrogel spacer to protect 
the rectal wall during radiotherapy of localized prostate cancer. Urology, 2013. 82(4): p. 963-8.

33. Lambin, P., J. Zindler, B.G. Vanneste, et al., Decision support systems for personalized and participative radiation oncology. Adv Drug Deliv Rev, 2017. 109: p. 131-153.

34. Vanneste, B.G., M. Pijls-Johannesma, L. Van De Voorde, et al., Spacers in radiotherapy treatment of prostate cancer: is reduction of toxicity cost-effective? Radiother Oncol, 2015. 114(2): p. 276-81. 


\section{Supplementary Material}

Supplementary Table S5.1: An overview of the patient characteristics.

\begin{tabular}{l|ll} 
Characteristic & Subgroup & Number of patients (\%) \\
\hline Risk group & Low & $2(12.5 \%)$ \\
& Intermediate & $7(43.75 \%)$ \\
& High & $7(43.75 \%)$ \\
\hline IRS & Balloon & $8(50 \%)$ \\
& Hydrogel & $8(50 \%)$ \\
\hline
\end{tabular}

IRS: Implantable rectum Spacer

Supplementary Table S5.2: An overview of the constraints set during treatment planning (given dose)

\begin{tabular}{l|ll} 
Structures & Constraints & \\
\hline Planning Target Volume & V70 $>95 \%$ & \\
Rectum Volume & V70 $=0 \%$ & Mean dose $<40$ Gy \\
Anal Canal Volume & V74 $=0 \%$ & V65 $<20 \%$ \\
Anorectum Volume & V54 $<50 \%$ & \\
Bladder Volume & V70 $=0 \%$ &
\end{tabular}

Gy: Gray, Vxx: percentage of volume receiving more than $x x G y$

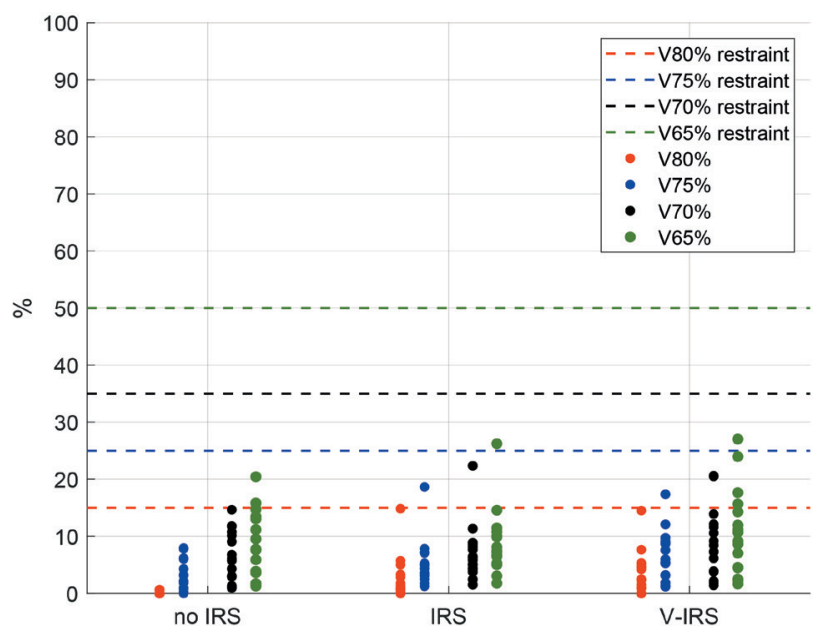

Supplementary Figure S5.1: The Vxx\% is the percentage of the bladder volume receiving at least $x x$ Gray (Gy). This figure shows the V80\%, V75\%,V70\% and V65\% for each of the patients included in the study after the dose was optimized by the isotoxic model. For all treatment plans, the bladder dose remains within the given limits. 

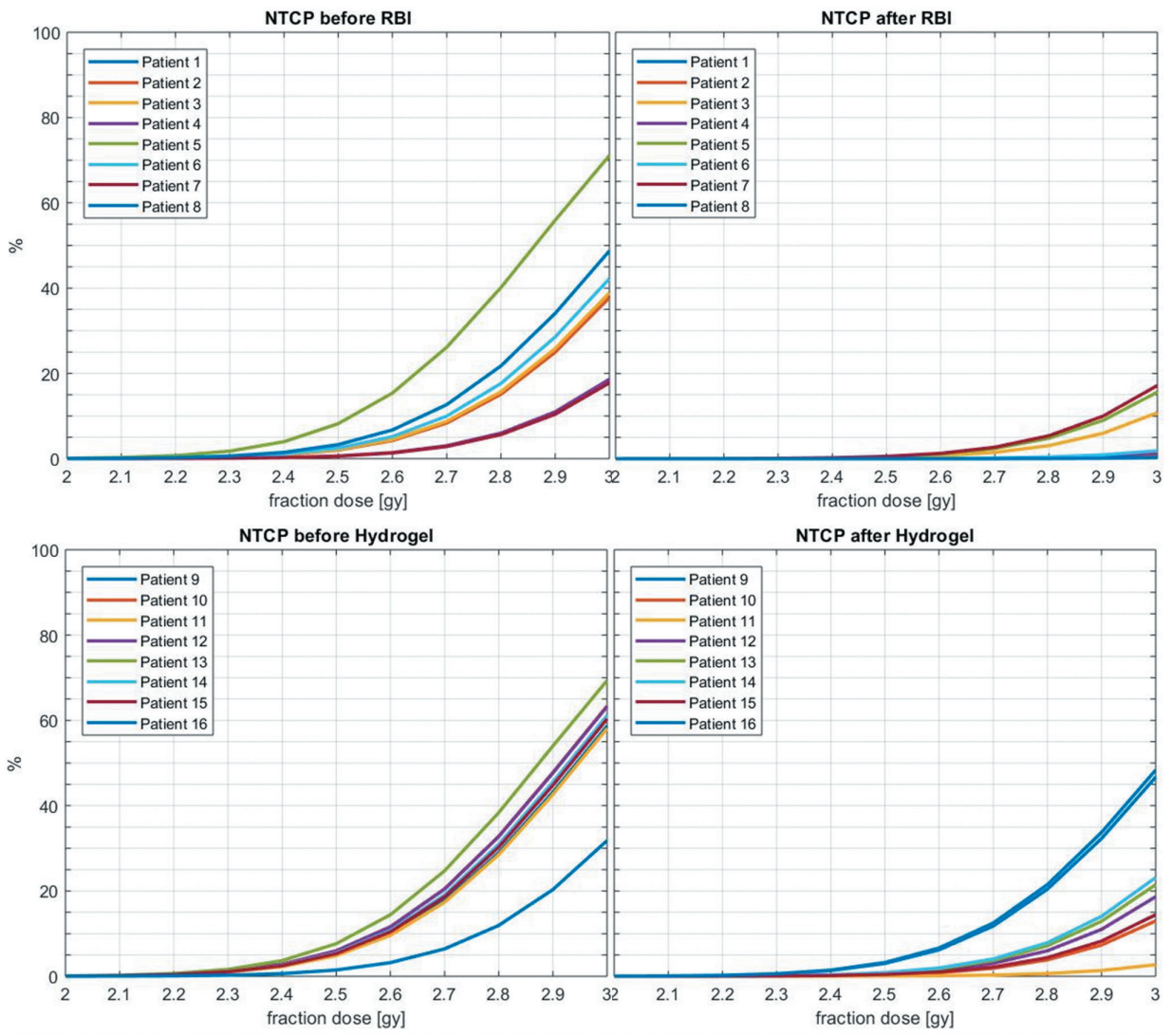

Supplementary Figure S5.2 the normal tissue complication probability (NTCP) predictions as function of the fraction dose for each individual patient before and after the placement of an implantable rectum spacer (IRS). The first 8 patients received a rectum balloon implant (RBI) and the second 8 received a hydrogel spacer. 

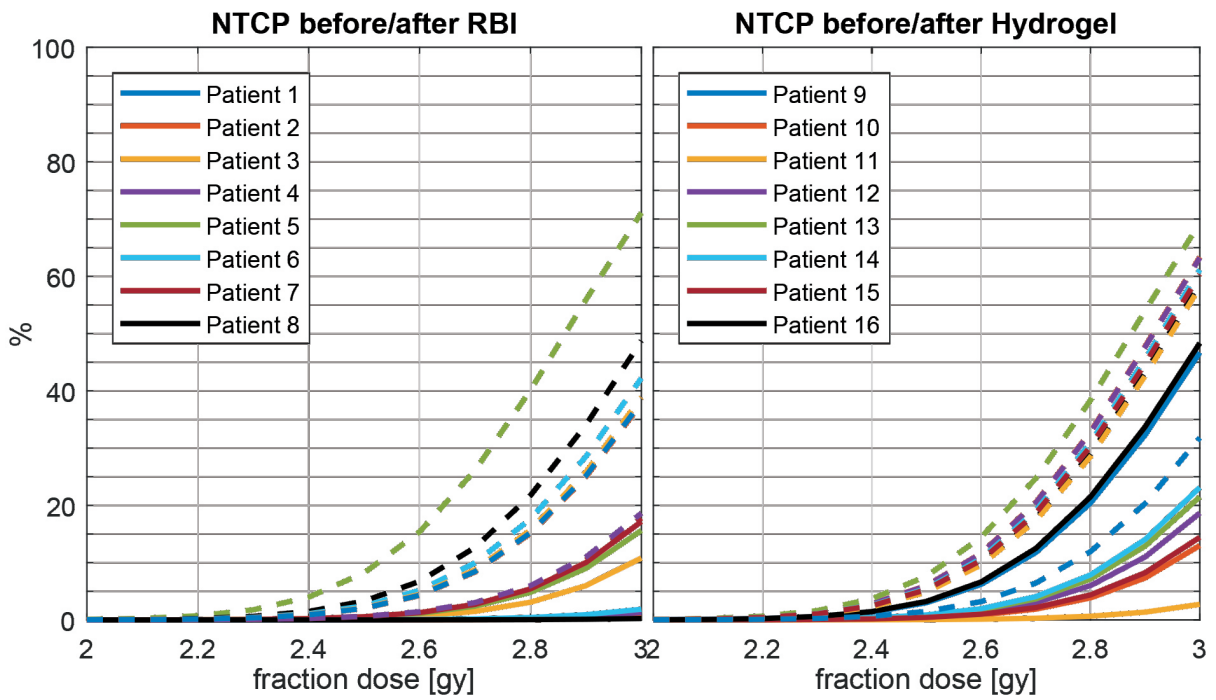

Supplementary Figure S5.3: The normal tissue complication probability (NTCP) predictions as function of the fraction dose for each individual patient before and after the placement of an implantable rectum spacer (IRS). The first 8 patients received a rectum balloon implant (RBI) and the second 8 received a hydrogel spacer. The dashed lines are before IRS placement, the solid lines after.

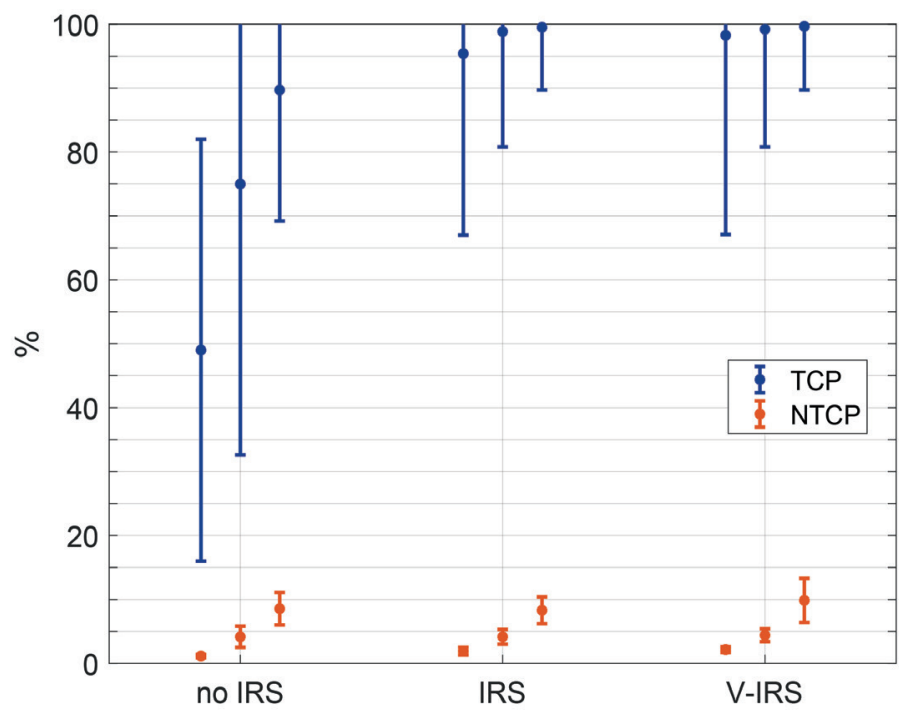

Supplementary Figure S5.4: The median tumour control probability (TCP) and range, as well as the median normal tissue complication probability (NTCP) and range, for the patients without implantable rectum spacer (IRS), with IRS and with the virtual IRS (V-IRS). Per group, the NTCP limit of the iso-toxic model is set to be 2.5, 5 and $10 \%$. 

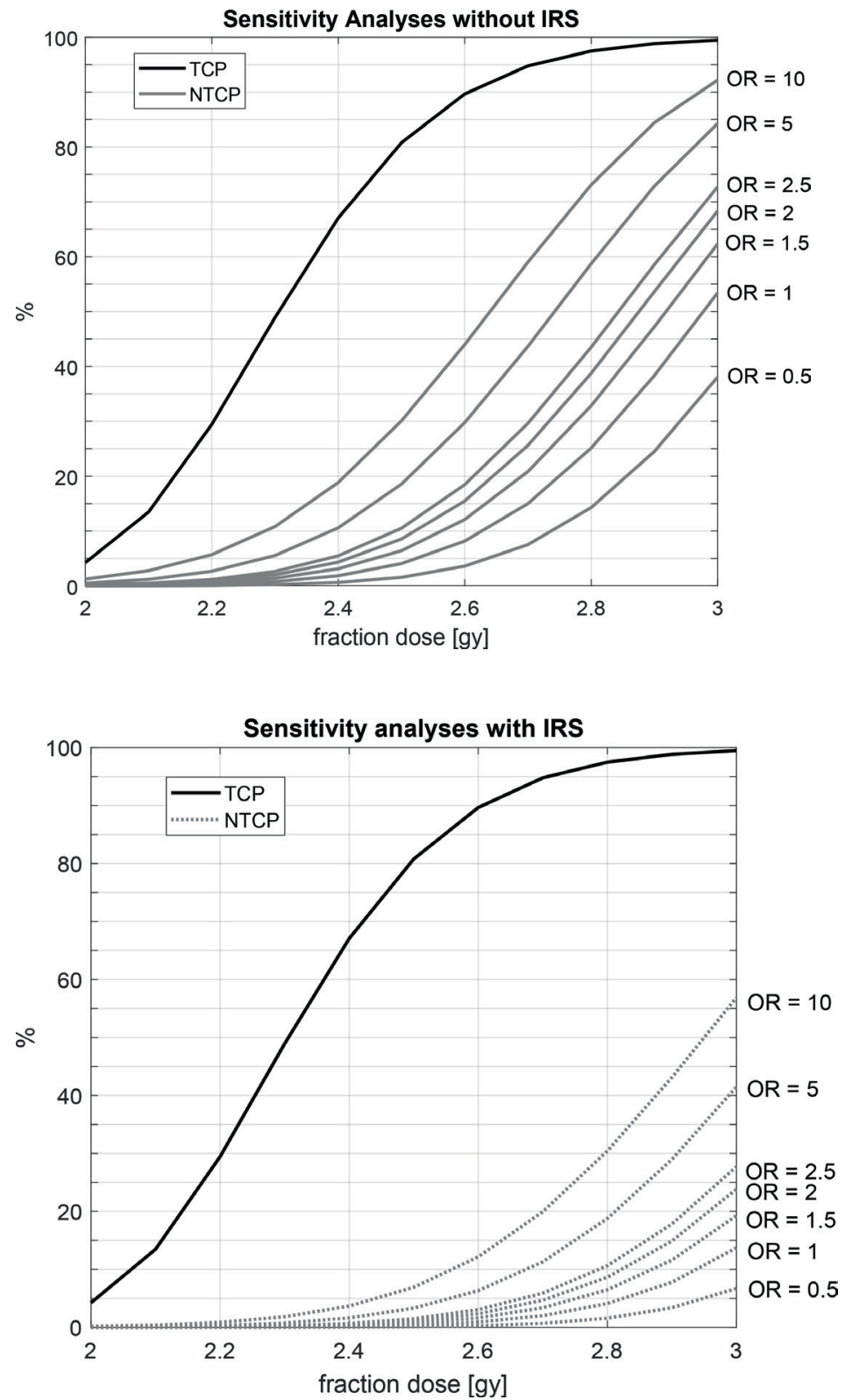

Supplementary Figure S5.5: To demonstrate the effect of the odds ratios (ORs) single nucleotide polymorphisms (SNPS), the OR's of the SNPS were artificially varied between 0.5 and 10, and the resulting normal tissue complication probability (NTCP) was calculated for the different fraction doses. Since the tumour control probability (TCP) is not effected by the SNPs, this remains the same. The top plot shows the results without implantable rectum spacer (IRS), the bottom one with. The Minor Allele Frequency for all these ORs was assumed to be 0.05 . 

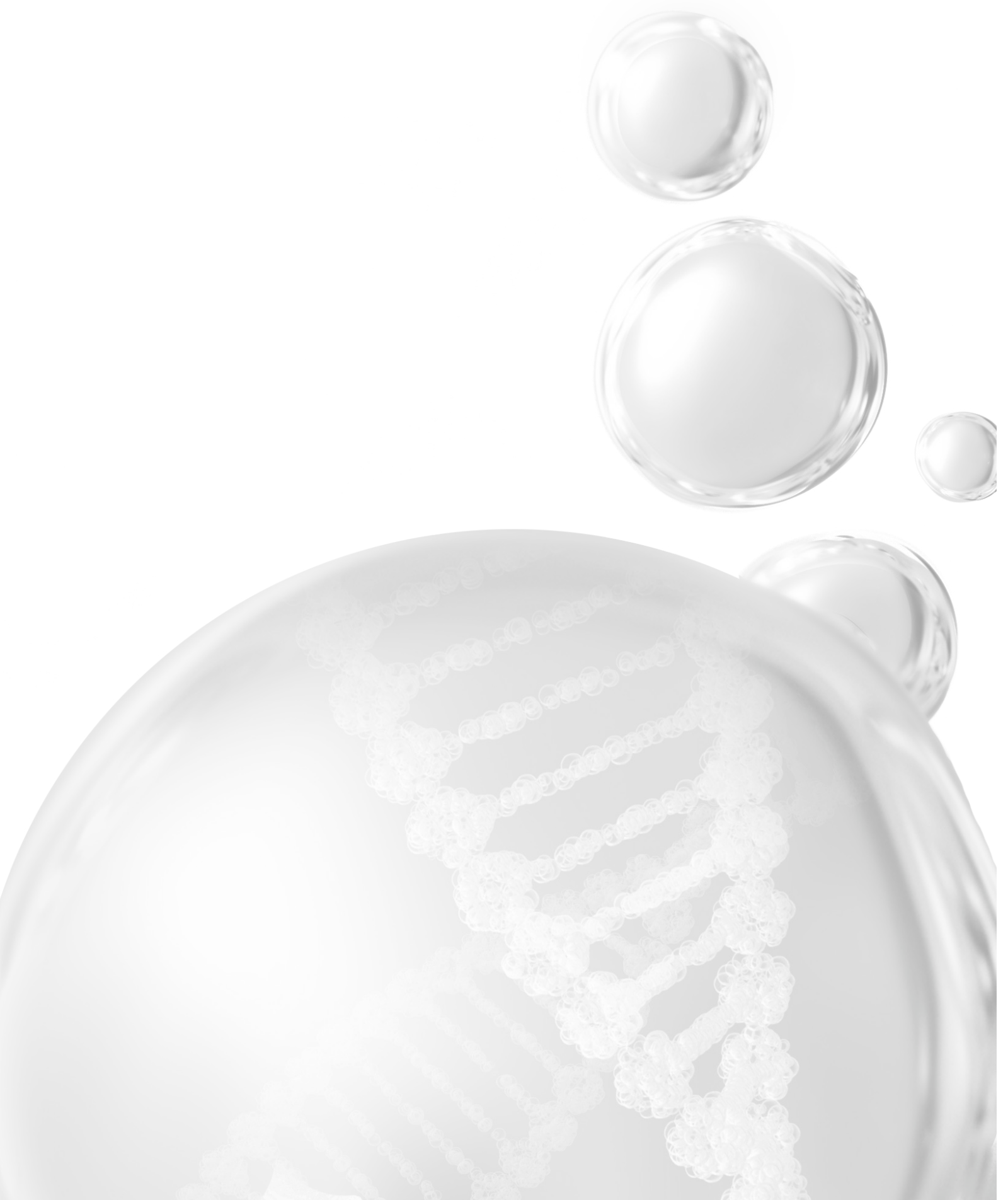


\section{Chapter 6}

\section{Influence of rectal spacer dynamics on anorectal dose and late rectal complication risk}

Authors:

Ben Vanneste, Yvonka van Wijk, Ludy Lutgens, Evert van Limbergen, Emile van Lin, Kees van de Beek, Philippe Lambin, Aswin Hoffmann

Adapted from:

"Dynamics of rectal balloon implant shrinkage in prostate VMAT: Influence on anorectal dose and late rectal complication risk."

Strahlenther Onkol. 2018 Jan; 194(1):31-40.

DOI: 10.1007/s00066-017-1222-x 


\section{Abstract}

Purpose: To assess the effect of a shrinking rectal balloon implant (RBI) on the anorectal dose and complication risk during the course of moderately hypofractionated prostate radiotherapy.

Methods: In 15 patients with localized prostate cancer, an RBI was implanted. A weekly kilovolt cone-beam computed tomography (CBCT) scan was acquired to measure the dynamics of RBI volume and prostate-rectum separation. The absolute anorectal volume encompassed by the 2 Gy equieffective 75 Gy isodose (V75Gy) was recalculated as well as the mean anorectal dose. The increase in estimated risk of grade 2-3 late rectal bleeding (LRB) between the start and end of treatment was predicted using nomograms. The observed acute and late toxicities were evaluated.

Results: A significant shrinkage of RBI volumes was observed, with an average volume of $70.4 \%$ of baseline at the end of the treatment. Although the prostaterectum separation significantly decreased over time, it remained at least $1 \mathrm{~cm}$. No significant increase in V75Gy of the anorectum was observed, except in one patient whose RBI had completely deflated in the third week of treatment. No correlation between mean anorectal dose and balloon deflation was found. The increase in predicted LRB risk was not significant, except in the one patient whose $\mathrm{RBI}$ completely deflated. The observed toxicities confirmed these findings.

Conclusions: Despite significant decrease in RBI volume the high-dose rectal volume and the predicted LRB risk were unaffected due to a persistent spacing between the prostate and the anterior rectal wall. 


\section{Introduction}

External beam radiation therapy (EBRT) is an effective curative treatment option for patients with localized adenocarcinoma of the prostate compared to surgery ${ }^{[1]}$. Increasing radiation dose is associated by increased control however this is correlated with an increased potential risk of gastro-intestinal (GI) toxicity, with possibly a decrease in the quality of life ${ }^{[2-4]}$. Despite the development of advanced treatment techniques like intensity-modulated radiation therapy, volumetric arc therapy, and image-guided radiotherapy, sparing of the rectal wall is a prerequisite for safe delivery of high doses to the prostate. This makes the rectum the dominant dose-limiting organ at risk in prostate EBRT. To spare the rectum, artificial spacing material has been used for insertion into the retro-prostatic space. Implantable rectum spacers (IRS) separate the anterior rectal wall from the prostate by creating an artificial distance between these organs. As such they reduce the dose exposure to the rectum, consequently decreasing the risk of GI toxicity. Different types of IRS exist, all of which are implanted through a transperineal approach. Hyaluronic acid $^{[5]}$ and collagen implants ${ }^{[6]}$ are physiologically compounds made of substances that are naturally present in the human body. Potential side effects for transmission of infectious agents or immunological reactions have been reported ${ }^{[7]}$. Therefore, commercially available spacers based on polyethylene glycol (PEG) hydrogels ${ }^{[8]}$ and biodegradable saline-filled rectal balloon implants $(\mathrm{RBI})^{[9]}$ have been developed. Recently Wolf and co-workers compared PEG and RBI spacer technologies in 59 prostate cancer patients undergoing radiation treatment and concluded that the RBI was superior in reducing rectum dose, whereas the PEG-hydrogel spacer had a better volume consistency with respect to the duration of treatment ${ }^{[10]}$. They reported an early and sudden RBI volume decline in 4 out of 16 patients, and an average volume loss of $>50 \%$ in the remaining 12 patients during the full course of treatment over 8 weeks of a normofractionated radiotherapy regimen comprising 41 fractions.

The aim of our study was to evaluate the RBI volume stability and the dosimetric effect of $\mathrm{RBI}$ volume shrinkage on the anorectum and to estimate the 3-year risk of grade 2-3 late rectal bleeding (LRB) during the course of a moderately hypofractionated EBRT regimen comprising 28 fractions. We tested the hypotheses that despite of an expected RBI volume decrease over time there is no significant increase in the absolute anorectal volume encompassed by the 2 Gy equieffective dose of 75 Gy (V75Gy) and in the mean anorectal dose, both of which are considered relevant parameters for predicting LRB. We also tested the hypothesis that there is no correlation between RBI deflation and mean anorectal dose. Additionally we hypothesized that the predicted increase in risk of LRB resulting from the volume decrease of the RBI is insignificant. Furthermore, we postulated that this predicted status quo of LRB risk can be explained by a persistent prostate-rectum separation 
of at least $1 \mathrm{~cm}$ during the whole course of treatment. Finally, we reported the observed acute and-as far as possiblelate toxicities.

\section{Materials and methods}

Patient selection

After approval by the institutional review board number 14-38-03/09internal-6335) 15 consecutive prostate cancer patients were prospectively included in this study between June 2015 and March 2016. Patients with a histologically confirmed, localized adenocarcinoma of the prostate were enrolled in this study to receive an $\mathrm{RBI}$ (BioProtect Ltd, Israel). All patients had signed an informed consent. The patient and tumor characteristics are summarized in Table 6.1. Patients who had been classified as intermediaterisk were prescribed additional neo-adjuvant hormonal therapy for 6 months ${ }^{[11]}$. The high-risk patients were offered an additional 1.5 years hormonal therapy in extent of the 6 months neo-adjuvant therapy after EBRT. All patients underwent magnetic resonance imaging (MRI) to exclude extraprostatic spread. Dorsal extra-prostatic disease extension (stage T3a/4) was an exclusion criteria, as well as distant metastatic disease, inflammatory bowel disease and previous pelvic EBRT.

Table 6.1 - Patient $(N=15)$ and tumor characteristics

\begin{tabular}{|c|c|}
\hline Parameter name & Value \\
\hline Age (years; median [range]) & $72[63-77]$ \\
\hline \multicolumn{2}{|c|}{ Prognostic risk group*: no. of patients) } \\
\hline Low-risk & $1(7 \%)$ \\
\hline Intermediate-risk & $5(33 \%)$ \\
\hline High-risk & $9(60 \%)$ \\
\hline
\end{tabular}

* Low-risk: no risk factors: PSA <10 $\mathrm{ng} / \mathrm{ml}$; Gleason score <7; cT-stage <2b; Intermediate-risk: PSA 10-20 ng/ml and/or Gleason score $=7$ or cT-stage $=2 \mathrm{~b} / \mathrm{c}$; High-risk: PSA $>20 \mathrm{ng} / \mathrm{ml}$ or Gleason score $>7$ or cT-stage $>2 \mathrm{~b} / \mathrm{c}$.

RBI implantation procedure

An RBI was implanted in these patients between the prostate and the anterior rectal wall 7-10 days prior to the start of EBRT. The injection technique has been described in detail previously ${ }^{[12]}$. A short general anesthesia is preferred at our department. However, the implantation procedure can be also performed under local or spinal anesthesia. Firstly, 4 fiducial markers (PolyMark ${ }^{\mathrm{TM}}, \mathrm{CIVCO}$, Orange City, USA) were implanted intraprostatically for daily position verification. The $\mathrm{RBI}$ was implanted transperineally under bi-plane transrectal ultrasonography guidance. A bubble-free (sterile) saline solution was used to fill and inflate the $\mathrm{RBI}$. The saline solution was mixed with approximately $1.5 \mathrm{~cm}^{3}$ iodinated contrast 
medium to enhance the visualization of the RBI on computed tomography (CT) scans. The injected volume was varied, depending on the volume of the prostate. Because the RBI should not be filled to achieve a prostate-rectum separation larger than $30 \mathrm{~mm}$ we adapted the volume of the RBI to the volume of the prostate: small prostates $(<35 \mathrm{cc}$ ) do not need the maximum RBI volume to guarantee a prostaterectum separation of at least $1 \mathrm{~cm}$, which is considered as a conclusive spread ${ }^{[13]}$.
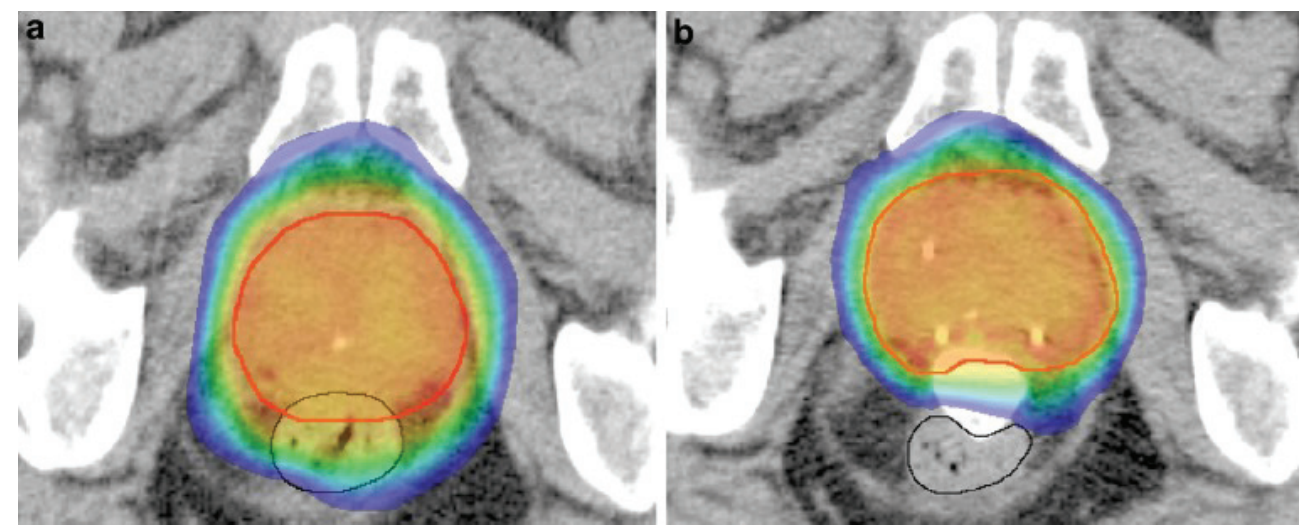

Figure 6.1. Color-wash isodose distribution projected on an axial CT slice before (a) and after $R B I$ implantation (b) in the same patient with the planning target volume in red. Without RBI (a), the high-dose region $>80 \%$ (green isodose) overlaps with the entire ventral part of the rectum (black line), whereas with the $R B I$ in situ (b) the rectum is exposed to a dose $<65 \%$ (blue isodose)

\section{Treatment planning}

Each patient underwent a CT scan and MRI scan 5-7 days after RBI implantation in supine position with a slice thickness of $3 \mathrm{~mm}$ for treatment planning and target volume delineation purposes, respectively. One hour prior to image acquisition patients were instructed to first empty their bladder, then drink $300 \mathrm{ml}$ of water to have a full bladder, and empty their bowel. No use of laxative was recommended. The CT and MRI scans (balanced turbo field-echo-sequence with isotropic $0.5 \mathrm{~mm}$ in-plane resolution) were co-registered based on the fiducial markers.

Delineation of the prostate (CTV = clinical target volume) was performed on the T2-weighted MRI scan, while the RBI and the organs at risk were delineated on the CT scan (Figure 6.1). In case the CT and MR images showed different prostate shapes and volumes (e.g. due to differences in rectal filling), the MR imaging was repeated. The first planning target volume (PTV1) was constructed according to the institutional protocol by expanding the CTV with 10, 7 and $6 \mathrm{~mm}$ in cranial-caudal, anterior-posterior, and left-right direction respectively. A second PTV (PTV2) was defined as a $5 \mathrm{~mm}$ isotropic expansion of the CTV, with exclusion of the anorectum 
and bladder. All treatment plans were designed for dose delivery with a volumetric modulated arc technique (VMAT) using $10 \mathrm{MV}$ photon beams (Eclipse Version ICD10, Varian Medical Systems Inc., Palo Alto, USA). The prescribed dose to PTV1 and PTV2 was 65.8 and $70 \mathrm{~Gy}$, in 28 fractions of 2.35 and $2.5 \mathrm{~Gy}$, respectively ${ }^{[14]}$. With $\alpha / \beta=3$ Gy for late rectal toxicity, the maximum 2 Gy equieffective dose (EQD23) in the anorectum for this type of plan is $77 \mathrm{~Gy}^{[15]}$. No density override for the iodinecontaining RBI was performed, because it was assumed that the contrast medium that was present in the RBI at the time of CT scanning and treatment planning remained present during treatment delivery. The overall treatment time was 7 weeks, at 4 fractions a week. The dose-volume constraints fulfilled the institutional protocol, which is based on the QUANTEC guidelines ${ }^{[16]}$. All patients underwent daily $X$-ray based position verification and repositioning based on the intraprostatic fiducial markers.

\section{RBI volume and distance dynamics}

To assess the volume stability of the RBI over time, a weekly kilovolt conebeam computed tomography (CBCT) scan was acquired in treatment position prior to irradiation, respectively at fractions 1, 7, 11, 15, 19, 23, and 27. The resulting 105 CBCT scans were imported into the treatment planning system for delineation and subsequent 3D volumetry of the RBI. Furthermore, the minimum distance between the prostate and the anterior rectal wall was measured at mid-prostate level in anterior-posterior direction. Two observers independently delineated the $\mathrm{RBI}(\mathrm{RG}$ and $\mathrm{BV}$ ) and the anorectum (DH and BV). The anorectal structure consists of the rectum and the analcanal. The rectum was delineated from the top of the anal-canal up to the recto-sigmoid flexure. The anal-canal was considered as the distal $3 \mathrm{~cm}$ of the anorectum. Six to nine months after RBI implantation an MRI scan was acquired to evaluate the biodegradability of the RBI.

\section{Anorectal dose estimation}

To assess the dosimetric consequences of RBI shrinkage on the anorectum, the planned dose distribution of the three patients with the largest observed $\mathrm{RBI}$ volume reduction was recalculated on the weekly acquired CBCT images, while keeping all planning parameters (e.g. beam arrangement, field size, fluence maps, monitor units) the same as for the initial, CT-based treatment plan. The DVH for the anorectum obtained from the treatment plan was converted into EQD23 using the Withers formula ${ }^{[17]}$ :

$$
E Q D 2=D \cdot \frac{d+^{\alpha} / \beta}{2+^{\alpha} / \beta}
$$


where $\alpha / \beta=3$ Gy for late rectal toxicity, $D$ is the total dose, and $d$ is the dose per fraction. All dose(-volume) parameters were obtained from the EQD23-converted DVH. The absolute V75Gy of the anorectum was compared between the CT and CBCT plans: in the 3 cases with the largest RBI volume decrease the weekly CBCT images were compared. For the remaining patients, only the CBCT images of the last week (CBCT27) were used for comparison of the anorectal V75Gy against the CT-based plan. The EQD23-converted mean anorectal dose was calculated for the planning $\mathrm{CT}$ and the final CBCT for all patients.

\section{Complication risk estimation}

To assess the effect of the RBI volume decrease on the 3-year risk of grade 2-3 LRB, a set of previously published multifactorial nomograms were used ${ }^{[18]}$. These nomograms use clinical parameters (use of anticoagulants, hormonal therapy, or anti-hypertensives; pelvic node irradiation; presence of diabetes or haemorrhoids; and a history of pre-RT abdominal surgery), in addition to dosimetric parameters (mean rectal dose and the percentage of the anorectum volume receiving at least an EQD23 of $75 \mathrm{~Gy}$ ) to predict the risk of late rectal bleeding. The nomograms were applied to the initial treatment plans and the plans performed on the final CBCT images for each patient. The results were used to estimate the largest change in predicted complication risk due to shrinkage of the RBI.

\section{Observed Toxicity Assessment}

The complications were recorded in terms of Common Terminology Criteria for Adverse Events (Version 4.03). Acute gastro-intestinal (GI) and genitourinary (GU) toxicities were scored in the 2 nd, 4 th, 6 th week of treatment and 3 months after its completion. Late toxicities were scored in the 6th, 9th, and - in case if possible -12 th, 18th months after treatment completion.

\section{Statistical analysis}

The statistical analyses were carried out using the Statistics Toolbox of MATLAB (Version 10.2, The MathWorks, Natick, USA) software. The pairedsamples Wilcoxon signed rank test was applied to test for a significant decrease in volume of the RBI on weekly acquired CBCT scans, and for a significant decrease in distance between prostate and rectum. This test was also applied to test for a significant increase in predicted complication risk between the first and last fraction. All statistical tests were one-sided, with $\mathrm{P}<0.05$ considered to be statistically significant. 
Table $6.2-R B I$ volume and minimum prostate-rectum distance dynamics

RBI volume

( $\mathrm{cm}^{3} ;$ median [range])

\section{Planning $C T$}

CBCT 1

CBCT 7

CBCT 11

CBCT 15

CBCT 19

CBCT 23

CBCT 27

\begin{tabular}{|ll}
$20.0[12.9-22.6]$ & $2.3[1.9-2.9]$ \\
$19.6[12.8-21.7]$ & $2.2[1.8-2.8]$ \\
$16.0[12.8-20.7]$ & $2.0[1.7-2.5]$ \\
$15.8[11.7-20.7]$ & $1.9[1.6-2.4]$ \\
$15.6[11.7-20.6]$ & $1.9[1.5-2.4]$ \\
$14.5[11.3-20.0]$ & $1.9[1.3-2.4]$ \\
$14.0[9.6-19.8]$ & $1.7[1.1-2.3]$ \\
$11.9[6.3-19.8]$ & $1.4[1.1-2.3]$
\end{tabular}

Prostate-rectum distance

(cm; median [range])

Abbreviations: $R B I=$ rectal balloon implant; $C B C T$ = cone-beam computed tomography

\section{Results}

$\mathrm{RBI}$ volume and distance stability

The median injected and delineated RBI volumes were $17.0 \mathrm{~cm}^{3}$ [range: 9-17 $\mathrm{cm}^{3}$ ] and $20.0 \mathrm{~cm}^{3}$ [range: $12.9-22.6 \mathrm{~cm}^{3}$ ], respectively. Volume differences are explained by the fact that the delineated RBI structure comprises both the injected saline solution and the RBI envelope. Two patients were excluded from the stability analysis, because in one patient $C B C T$ scans were missing due to a protocol violation and in another patient the RBI had disappeared on CBCT in the third week of treatment as no contrast medium could be detected. For the remaining 13 patients, the descriptive statistics of the RBI volume dynamics are summarized in Table 6.2 and depicted in Figure 2. The median RBI volumes at fractions 1, 15 and 27 were 19.6 [range: $12.8-21.7$ ], 15.6 [range: $11.7-20.6$ ], and $11.9 \mathrm{~cm}^{3}$ [range: $6.3-19.8$, respectively. The weekly decrease in absolute RBI volume was significant for all time points, with an average volume loss of $29.6 \%$ at fraction 27 relative to baseline. The largest relative volume decrease occurred during the first week (i.e. between fraction 1 and 7), and after fraction 19 (Figure 6.2). The median volume loss relative to baseline was 25\% [range: $5.7-54.9 \%$ ]. The descriptive statistics of the prostate-rectum distance dynamics are also summarized in Table 6.2. Although the weekly decrease of minimum prostate-rectum distance was significant for all time points, this distance remained greater than $1 \mathrm{~cm}$ at all times. 


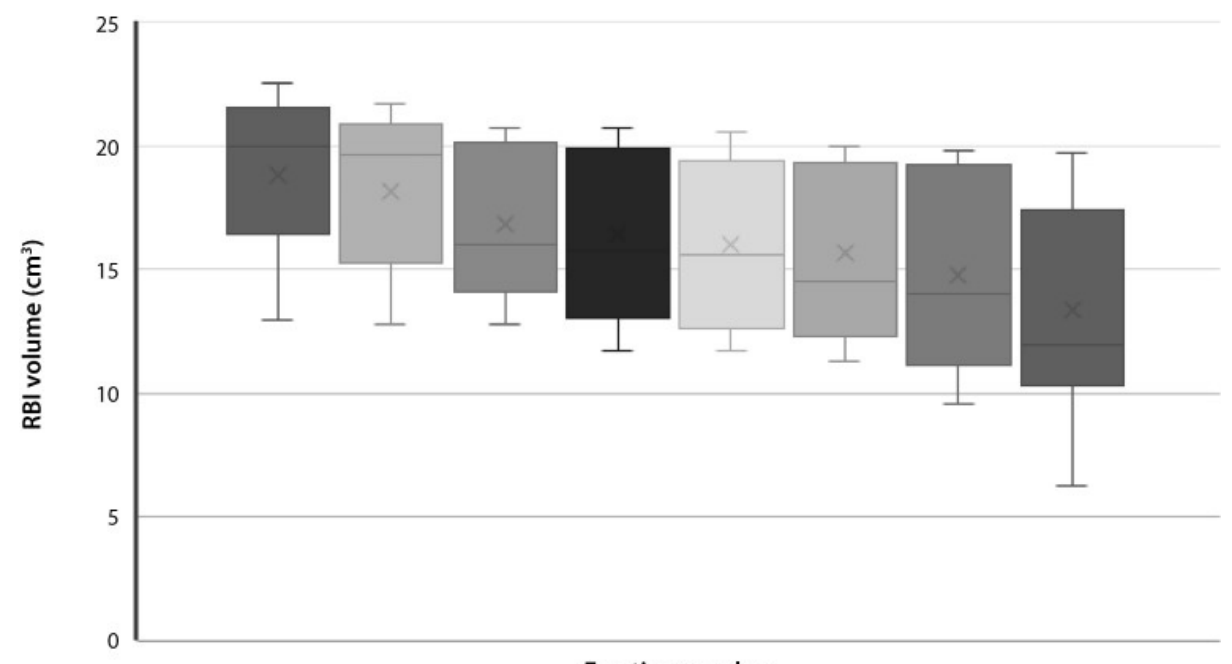

Fraction number

$\square$ CT/MRI $\square 1 \square 7 \square 11 \square 15 \square 19 \square 23 \square$ Fraction number

Figure 6.2. A box and whisker plot of the delineated rectal balloon implant (RBI) volumes during a full treatment course of 28 fractions in 13 patients (grey dashed lines) observed by weekly cone-beam computed tomography (CT) images

\section{$\mathrm{RB}$ I resorption}

Six to nine months after completion of EBRT a residual rest of the RBI envelope was visible on T2-weighted MRI in only 1 out of 15 (7\%) patients; no contrast medium or saline solution could be observed. Neither space-occupying effects nor complications (infections, perforations, fibrosis) were observed in any of the patients.

Table 6.3 -Descriptive statistics or treatment plans based on the planning CT scan and the CBCT scan of fraction 27

\begin{tabular}{l|ll} 
& \multicolumn{1}{l}{$\begin{array}{l}\text { Planning CT } \\
\text { median [range] }\end{array}$} & $\begin{array}{l}\text { CBCT27 } \\
\text { median [range] }\end{array}$ \\
\hline Rectal volume (cC) & $88.7[56.1-187.4]$ & $68.65[45.3-200.8]$ \\
Mean rectal dose (Gy) & $26.2[10.9-34.6]$ & $33.9[19.3-38.7]$ \\
V75Gy (cc) & $0.1[0.0-1.6]$ & $0.7[0.0-5.9]$ \\
Complication risk (\%) & $4.1[3.8-11.9]$ & $4.5[3.9-11.6]$ \\
\hline
\end{tabular}

CT computed tomography, CBCT cone-beam computed tomography, V75Gy volume receiving at least a 2 Gy equieffective dose (EQD23) of 75 Gy, Mean rectal dose mean EQD23 dose in the anorectum 


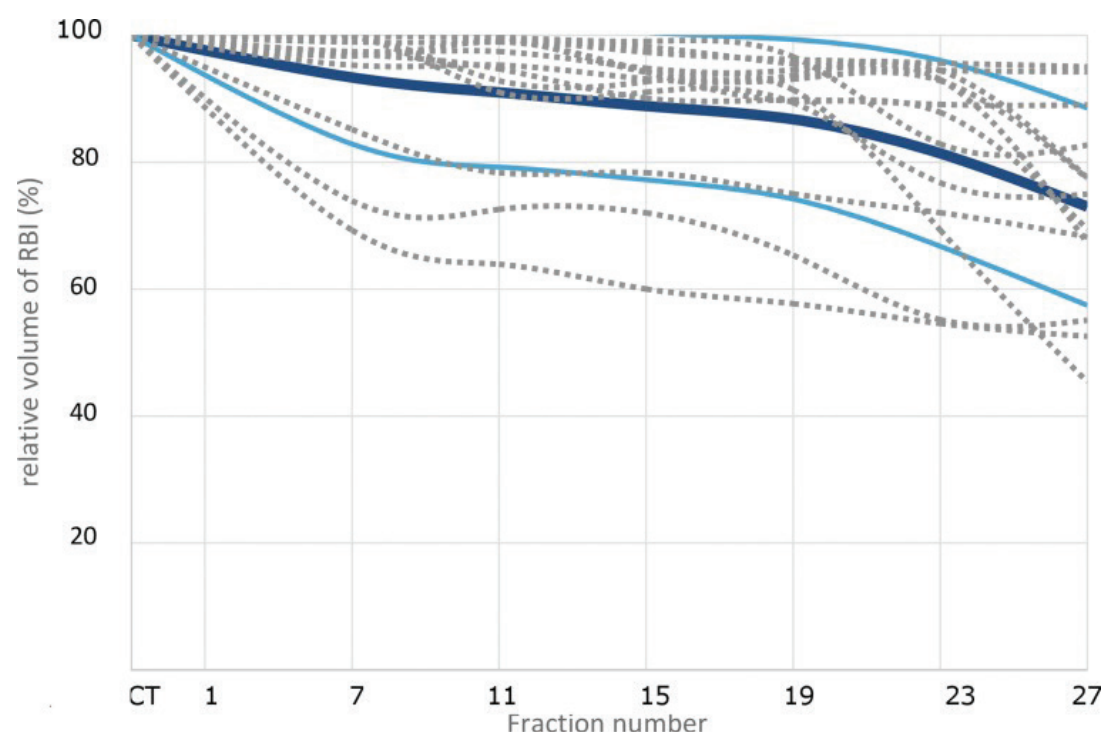

Figure 6.3. Relative volume dynamics of rectal balloon implant during a full treatment course of 28 fractions in 13 patients (grey dashed lines) observed by weekly cone-beam computed tomography (CT) images. The mean values (dark blue) with the standard deviations (light blue) are presented

\section{Anorectal dosimetry}

In the patient whose RBI had disappeared on the CBCT scan in the third week of treatment, the V75Gy of the anorectum significantly increased from 0 to 4.3 and $5.9 \mathrm{~cm}^{3}$ at fraction 1, 11 and 27, respectively (Pat. 9, Figure 6.3). In the other two patients (nrs. 5 and 12) exhibiting the largest RBI volume decrease (45\% and $52 \%$ of the original volume, respectively) a significant increase in absolute V75Gy of the anorectum was only observed in the CBCT scan of fraction 23 (Pat. 5 and 12, Figure 6.3). Furthermore, the RBI deflation did not lead to significant increase in V75Gy ( $p=0.577)$. Among the remaining patients there was only one patient who showed an increase of V75Gy from $0 \mathrm{~cm}^{3}$ on the CT plan to $3.6 \mathrm{~cm}^{3}$ on the CBCT27 plan (Table 6.3). In 2 out of 11 patients the $\mathrm{V} 75 \mathrm{~Gy}$ remained 0 for both the CT and CBCT27 scans. In 6 patients even a slight decrease of V75Gy was determined. The increase in V75Gy for all patients except the one of whom the RBI disappeared was not significant $(P=0.57)$. The increase in mean anorectal dose was significant $(P=$ 0.02), however, no correlation was found between the mean anorectal dose and the volume of the RBI $(P=0.3)$. The correlation between the anorectal volume and the anorectal mean dose was significant $(P=0.005)$. 


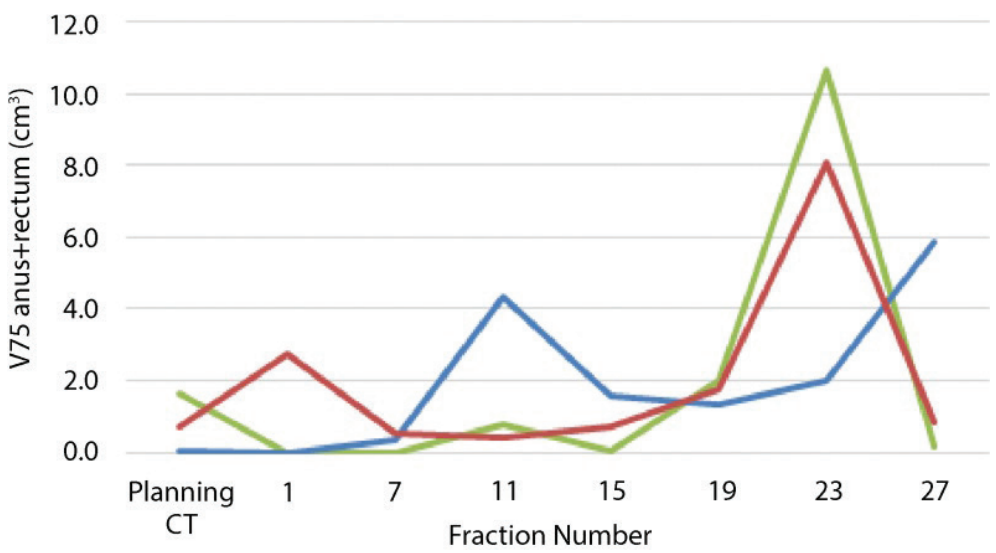

Pat. 5 -Pat. 9 Pat. 12

Figure 6.4. Dynamics of estimated absolute anorectal volume encompassed by the biological equivalent 75 Gy isodose during a full treatment course of 3 patients (numbers 5, 9, 12) exhibiting the largest volume decrease of the implanted rectal balloon. Patient 9 had the complete rectal balloon implant (RBI) shrinkage on cone-beam computed tomography (CBCT) of fraction 11: the V75Gy increased significantly. In the other two patients (5 and 12) a significant increase in absolute V75Gy of the anorectum was observed in only one CBCT scan (fraction 23) over the total treatment course.

\section{Risk of late rectal bleeding}

In the patient whose RBI had completely deflated in the third week of treatment (patient 9), the risk of LRB was predicted to increase from $4.0 \%$ to 9.8\%. For the two patients with the largest observed RBI shrinkage (patient 5 and 12) the predicted increase in LRB risk was $0 \%$ and $0.2 \%$, respectively (Figure 6.4). The largest increase in complication risk predicted for the remaining patients was $3.4 \%$, resulting from a RBI volume decrease of $32 \%$. The difference between the predicted risk of LRB at the start of treatment and the end of treatment, excluding the patient with the deflated RBI, was not significant $(P=0.07)$ (Figure 6.5). 


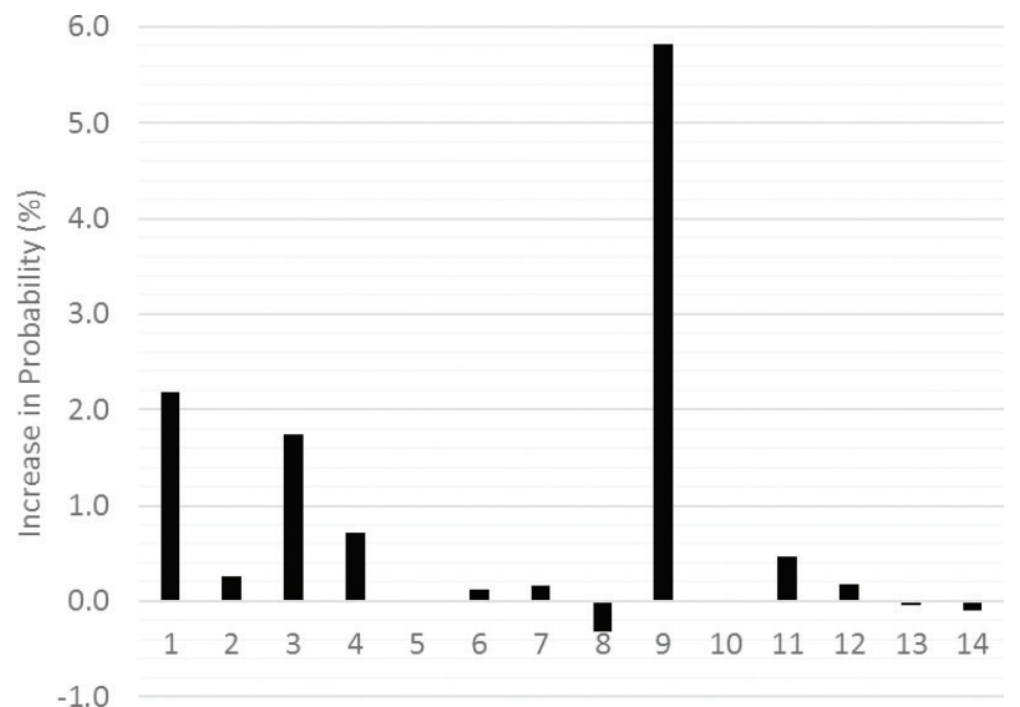

Patient Number

Figure 6.5. Increase in predicted probability (percentage points) of late rectal bleeding between the planning computed tomography (CT) and the last cone-beam CT (CBCT) scan (fraction 27) for each of the patients

\section{Observed toxicities}

No acute grade 3 or 4 toxicities were reported during treatment or 3 months after completion. Overall, 5 patients (33\%) experienced no toxicity at all, 6 patients (40\%) had grade $1 \mathrm{GU}$ toxicities, and 4 patients (27\%) had grade $2 \mathrm{GU}$ toxicities. During the course of therapy, in 4 patients (27\%) grade $1 \mathrm{Gl}$ toxicity was observed, but no grade 2 or more acute $\mathrm{Gl}$ toxicities.

No late grade $3 \mathrm{Gl}$ toxicity has been reported. Late grade 2 Gl toxicity was observed in 1 patient: the rectal bleeding started at 9 months after radiation. This was the patient whos RBI had completely deflated in the third week of treatment. Grade $1 \mathrm{GI}$ and $\mathrm{GU}$ late toxicities were reported in 2 patients.

\section{Discussion}

This study provides the first evaluation of RBI volume stability that is based on weekly CBCT measurements during the full course of EBRT. As previously reported in literature, an RBI volume decrease was expected over time. We analyzed the dosimetric consequences of this phenomenon and predicted the increase in risk of 3-years grade 2-3 late rectal bleeding resulting from a shrinking RBI to assess its potential clinical impact. Moreover, the observed acute and early late toxicities were adjusted. 
GI toxicity is the major treatment-related side effect in prostate cancer radiotherapy: the rates of acute and chronic Grade $\geq 2$ rectal toxicity has increased by dose-escalated EBRT (up to a dose of 78 Gy) compared with lower doses (e.g., $68 \mathrm{~Gy}$ ) from $3 \%$ to $20 \%$ and from $5 \%$ to $21 \%$, respectively ${ }^{[20-24]}$. Therefore, it is advantageous to push the rectal wall out of high-dose regions by implantation of an IRS device ${ }^{[25,26]}$. So far, most studies in literature have reported on the use of a PEGhydrogel as an IRS ${ }^{[27-31]}$. The RBI has some practical advantages ${ }^{[9]}$. First of all postimplant correction of the RBI position is possible; if the RBI is dispositioned, it can be easily deflated and replaced, whereas liquid spacers (PEG-hydrogels, hyaluronic acid, human collagen) do not permit any correction once being injected ${ }^{[9,12]}$. In addition, a chemical reaction is required to occur in PEG-hydrogels, which limits the implantation time. Furthermore, since the RBI inflates to a predetermined and predictable shape, the learning curve to obtain an adequate implant is less steep than for PEG-hydrogels. Additionally, due to the defined shape and homogenous hypodense signal, the RBI has an excellent CT visibility, which is most advantageous for treatment planning and CBCT-based evaluations. Besides, some amount of iodine contrast can be added to the saline to enhance the visualization of the RBI on CT and CBCT scans (Figure 6.1). Moreover, because the RBI is a closed system, there is no risk of air or hydrogel injection into vessels.

Nevertheless, also disadvantages of the RBI have been reported. Recently Wolf and colleagues described an early deflation effect ${ }^{[10]}$. They reported an average volume loss of $>50 \%$ during a full treatment course of 37-41 fractions ( 8 weeks). The volumes they estimated were mainly based on measurements of the diameters of the RBI on two orthogonal X-ray images and calculations by the volume formula for an ellipsoid cylinder. The measurements were only performed on CBCT scans of fraction 20 and 38. Data on the dynamics of the prostate-rectum separation over time is missing in their study. Wolf and colleagues only recalculated one treatment plan on a CBCT scan for a single patient whose RBI showed a significant volume loss of $58 \%$, and reported an increase of $9.2 \mathrm{~cm}^{3}$ for the rectum volume encompassed by the $95 \%$ isodose. We measured 3D volume changes on weekly CBCT scans and observed that a prostate-rectum separation of at least $1 \mathrm{~cm}$ is maintained during the full treatment course, except in the patient where the RBI deflated. The persistent spread of at least $1 \mathrm{~cm}$ means that also for other RT techniques like 3D conventional EBRT, intensity-modulated radiotherapy, or proton therapy, the sustained spread is considered as enough for protecting the anorectal structures ${ }^{[13]}$.

By evaluating the dose on the anorectal structure, we observed that the V75Gy of the anorectum steadily increased only in one patient whose RBI disappeared completely in the third week of treatment. In the 2 remaining patients where the RBI shrank most, the V75Gy changed significantly in only 2 CBCT scans during the whole treatment course, but not in the last CBCT scan. This effect was caused by 
a difference in rectal filling (inclusion of gas bubbles and/or stool). The distance between the prostate and anorectum was still enlarged due to the presence of the RBI. However, the distance between the prostate and anorectum at the level of the cranial part of the rectum (above the RBI) could decrease incrementally. This is especially the case when the rectal filling is increased dramatically, and when seminal vesicles are irradiated. In this situation, the cranial part of the rectum above the RBI anorectum could receive a higher dose. Further, the RBI could exert pressure on the rectum and thereby decrease the rectal volume being exposed to an intermediate and a high dose and increase the volume being exposed to low doses, with unknown clinical consequences. To reduce the low- and intermediate dose levels, a different treatment planning technique using either an arc technique with an avoidance region near the rectum or using strictly anterior and lateral beams would be required.

By applying multifactorial nomograms on the initial treatment plan and on the final treatment plan, the predicted increase in risk of late rectal bleeding was analyzed. This showed that for the patient with the deflated RBI, the risk considerably increased, emphasizing the effect of the device. For the patient whos RBI had completely deflated a significant increase in the risk of late rectal bleeding was predicted by the nomogram. This was confirmed by the late rectal bleeding event at 9 months after treatment. For the remaining patients there was no significant increase in predicted complication risk, suggesting that the decrease in RBI volume has little impact on the effectiveness of the RBI. Some fluctuations in predicted toxicity risk can be seen, but these are likely also due to the rectal filling. Indeed, besides the distance between the rectal wall and the prostate, the rectum size could be a possible predictor of GI toxicity ${ }^{[32-35]}$.

A previous study reported a sudden complete deflation of the RBI in 4 out of 16 patients three weeks after implantation ${ }^{[10]}$. In our series, one patient experienced such a sudden complete deflation three weeks after implantation. A possible explanation was a non-optimal positioning: the RBI was positioned more caudally than the others, with the tip of the RBI in the pelvic muscle. Because of this positioning an excessive force was required to inflate the RBI, which could have damaged the sealing mechanism. Another possible explanation of early deflation could be an excessive filling of the RBI (i.e., prostate-rectum separation larger than $30 \mathrm{~mm}$ ) with bursting and consequent loss of function: each RBI is handmade, and one has to be sure not to exceed the maximum volume allowed that is indicated on the label of the product.

A limitation of this study is the limited number of patients included. As this was a feasibility study, only 15 patients were included. Furthermore, there was no prior consensus on the level and window settings of the CBCT scans, which might have influenced the volumetric results. In addition, the CBCT scans were 
acquired at different time points and hence revealed different bladder and rectum filling, hence adding extra uncertainty to the comparison performed. Further, the nomograms used are not validated for patients treated with a RBI. More research is needed in larger patient cohorts to obtain more evidence. Finally, we evaluated weekly time points, and not daily, which latter could be more representative for the whole treatment.

The RBI was successfully implanted in all 15 patients. The mean RBI volumes revealed an average volume of $70.4 \%$ of baseline at the end of treatment. Despite the weekly RBI shrinkage to be significant, neither significant increase in absolute V75Gy of the anorectum nor in predicted LRB risk were observed over the full treatment course of our moderately hypofractionated EBRT regimen. Although the minimum prostate-rectum distance showed a significant decrease, it was at least $1 \mathrm{~cm}$ during the full treatment course, indicating that such spacing is sufficient to reduce the anorectal $V 75 G y$ of a treatment plan delivered by a volumetric modulated arc technique. In patients experiencing a complete deflation of the RBI, the absolute V75Gy of the anorectum is expected to increase significantly, and so is the predicted risk of late rectal bleeding, and the observed toxicity. We advise to acquire imaging by CBCT scans at regular times during the course of treatment to assess deflation dynamics of the RBI. Only when the prostate-rectum distance is decreasing under $1 \mathrm{~cm}$ a treatment plan adaptation is recommended.

\section{Acknowledgements}

The authors thank Renee Granzier, Debbie Herfs, Janneke Bovendeerd, and Marlies Lendfers for their help with the delineations and treatment planning on the cone-beam CT scans. 


\section{References}

1. Hamdy, F.C., et al., 10-Year Outcomes after Monitoring, Surgery, or Radiotherapy for Localized Prostate Cancer. N Engl J Med, 2016. 375(15): p. 1415-1424.

2. Fonteyne, V., et al., Rectal toxicity after intensity modulated radiotherapy for prostate cancer: which rectal dose volume constraints should we use? Radiother Oncol, 2014. 113(3): p. 398-403.

3. Musunuru, H.B., et al., Dose-escalation of five-fraction SABR in prostate cancer: Toxicity comparison of two prospective trials. Radiother Oncol, 2016. 118(1): p. 112-7.

4. Vanneste, B.G., et al., Chronic radiation proctitis: tricks to prevent and treat. Int J Colorectal Dis, 2015. 30(10): p. 1293-303.

5. Prada, P.J., et al., Transperineal injection of hyaluronic acid in anterior perirectal fat to decrease rectal toxicity from radiation delivered with intensity modulated brachytherapy or EBRT for prostate cancer patients. Int J Radiat Oncol Biol Phys, 2007. 69(1): p. 95-102.

6. Noyes, W.R., C.C. Hosford, and S.E. Schultz, Human collagen injections to reduce rectal dose during radiotherapy. Int J Radiat Oncol Biol Phys, 2012. 82(5): p. 1918-22.

7. Prendes, B.L., et al., Long-term effects of injection laryngoplasty with a temporary agent on voice quality and vocal fold position. Laryngoscope, 2012. 122(10): p. 2227-33.

8. Pinkawa, M., et al., Application of a spacer gel to optimize three-dimensional conformal and intensity modulated radiotherapy for prostate cancer. Radiother Oncol, 2011. 100(3): p. 436-41.

9. Melchert, C., et al., Interstitial biodegradable balloon for reduced rectal dose during prostate radiotherapy: results of a virtual planning investigation based on the pre- and post-implant imaging data of an international multicenter study. Radiother Oncol, 2013. 106(2): p. 210-4.

10. Wolf, F., et al., Comparison of two different rectal spacers in prostate cancer external beam radiotherapy in terms of rectal sparing and volume consistency. Radiother Oncol, 2015. 116(2): p. 221-5.

11. Denham, J.W., et al., Paradoxical metastatic progression following 3 months of neo-adjuvant androgen suppression in the TROG 96.01 trial for men with locally advanced prostate cancer. Radiother Oncol, 2013. 107(2): p. 123-8.

12. Vanneste, B.G.L., et al., Implantation of a biodegradable rectum balloon implant: tips, Tricks and Pitfalls. Int Braz J Urol, 2017. 43(6): p. 1033-1042.

13. Susil, R.C., et al., Effects of prostate-rectum separation on rectal dose from external beam radiotherapy. Int J Radiat Oncol Biol Phys, 2010. 76(4): p. 1251-8.

14. Kupelian, P.A., et al., Hypofractionated intensity-modulated radiotherapy (70 Gy at 2.5 Gy per fraction) for localized prostate cancer: Cleveland Clinic experience. Int J Radiat Oncol Biol Phys, 2007. 68(5): p. 1424-30.

15. Bentzen, S.M., et al., Bioeffect modeling and equieffective dose concepts in radiation oncology--terminology, quantities and units. Radiother Oncol, 2012. 105(2): p. 266-8.

16. Michalski, J.M., et al., Radiation dose-volume effects in radiation-induced rectal injury. Int J Radiat Oncol Biol Phys, 2010. 76(3 Suppl): p. S123-9.

17. Withers, H.R., H.D. Thames, Jr., and L.J. Peters, A new isoeffect curve for change in dose per fraction. Radiother Oncol, 1983. 1(2): p. 187-91.

18. Valdagni, R., et al., Is it time to tailor the prediction of radio-induced toxicity in prostate cancer patients? Building the first set of nomograms for late rectal syndrome. Int J Radiat Oncol Biol Phys, 2012. 82(5): p. 1957-66. 
19. Institute, N.C., Common Terminology Criteria for Adverse Events 4.03 (CTCAE). 2017: http://ctep.cancer.gov/ protocolDevelopment/electronic_applications/ctc.htm.

20. Al-Mamgani, A., et al., Update of Dutch multicenter dose-escalation trial of radiotherapy for localized prostate cancer. Int J Radiat Oncol Biol Phys, 2008. 72(4): p. 980-8.

21. Aluwini, S., et al., Hypofractionated versus conventionally fractionated radiotherapy for patients with prostate cancer (HYPRO): acute toxicity results from a randomised non-inferiority phase 3 trial. Lancet Oncol, 2015. 16(3): p. 274-83.

22. Ghadjar, P., et al., Patterns and predictors of amelioration of genitourinary toxicity after high-dose intensitymodulated radiation therapy for localized prostate cancer: implications for defining postradiotherapy urinary toxicity. Eur Urol, 2013. 64(6): p. 931-8.

23. Kuban, D.A., et al., Long-term results of the M. D. Anderson randomized dose-escalation trial for prostate cancer. Int J Radiat Oncol Biol Phys, 2008. 70(1): p. 67-74.

24. Zelefsky, M.J., et al., Incidence of late rectal and urinary toxicities after three-dimensional conformal radiotherapy and intensity-modulated radiotherapy for localized prostate cancer. Int J Radiat Oncol Biol Phys, 2008. 70(4): p. $1124-9$.

25. Mok, G., et al., Optimization of radiation therapy techniques for prostate cancer with prostate-rectum spacers: a systematic review. Int J Radiat Oncol Biol Phys, 2014. 90(2): p. 278-88.

26. Pinkawa, M., Spacer application for prostate cancer radiation therapy. Future Oncol, 2014. 10(5): p. 851-64.

27. Mariados, N., et al., Hydrogel Spacer Prospective Multicenter Randomized Controlled Pivotal Trial: Dosimetric and Clinical Effects of Perirectal Spacer Application in Men Undergoing Prostate Image Guided Intensity Modulated Radiation Therapy. Int J Radiat Oncol Biol Phys, 2015. 92(5): p. 971-977.

28. Pinkawa, M., et al., Hydrogel injection reduces rectal toxicity after radiotherapy for localized prostate cancer. Strahlenther Onkol, 2017. 193(1): p. 22-28.

29. Song, D.Y., et al., A multi-institutional clinical trial of rectal dose reduction via injected polyethylene-glycol hydrogel during intensity modulated radiation therapy for prostate cancer: analysis of dosimetric outcomes. Int J Radiat Oncol Biol Phys, 2013. 87(1): p. 81-7.

30. Strom, T.J., et al., A dosimetric study of polyethylene glycol hydrogel in 200 prostate cancer patients treated with high-dose rate brachytherapy+/-intensity modulated radiation therapy. Radiother Oncol, 2014. 111(1): p. 126-31.

31. Uhl, M., et al., Low rectal toxicity after dose escalated IMRT treatment of prostate cancer using an absorbable hydrogel for increasing and maintaining space between the rectum and prostate: results of a multi-institutional phase II trial. Radiother Oncol, 2013. 106(2): p. 215-9.

32. Chen, Z., et al., Dosimetric impact of different bladder and rectum filling during prostate cancer radiotherapy. Radiat Oncol, 2016. 11: p. 103.

33. Hedrick, S.G., et al., Validation of rectal sparing throughout the course of proton therapy treatment in prostate cancer patients treated with SpaceOAR((R)). J Appl Clin Med Phys, 2017. 18(1): p. 82-89.

34. Hoogeman, M.S., et al., Strategies to reduce the systematic error due to tumor and rectum motion in radiotherapy of prostate cancer. Radiother Oncol, 2005. 74(2): p. 177-85.

35. Huang, T.C., et al., Fractionated changes in prostate cancer radiotherapy using cone-beam computed tomography. Med Dosim, 2015. 40(3): p. 222-5. 

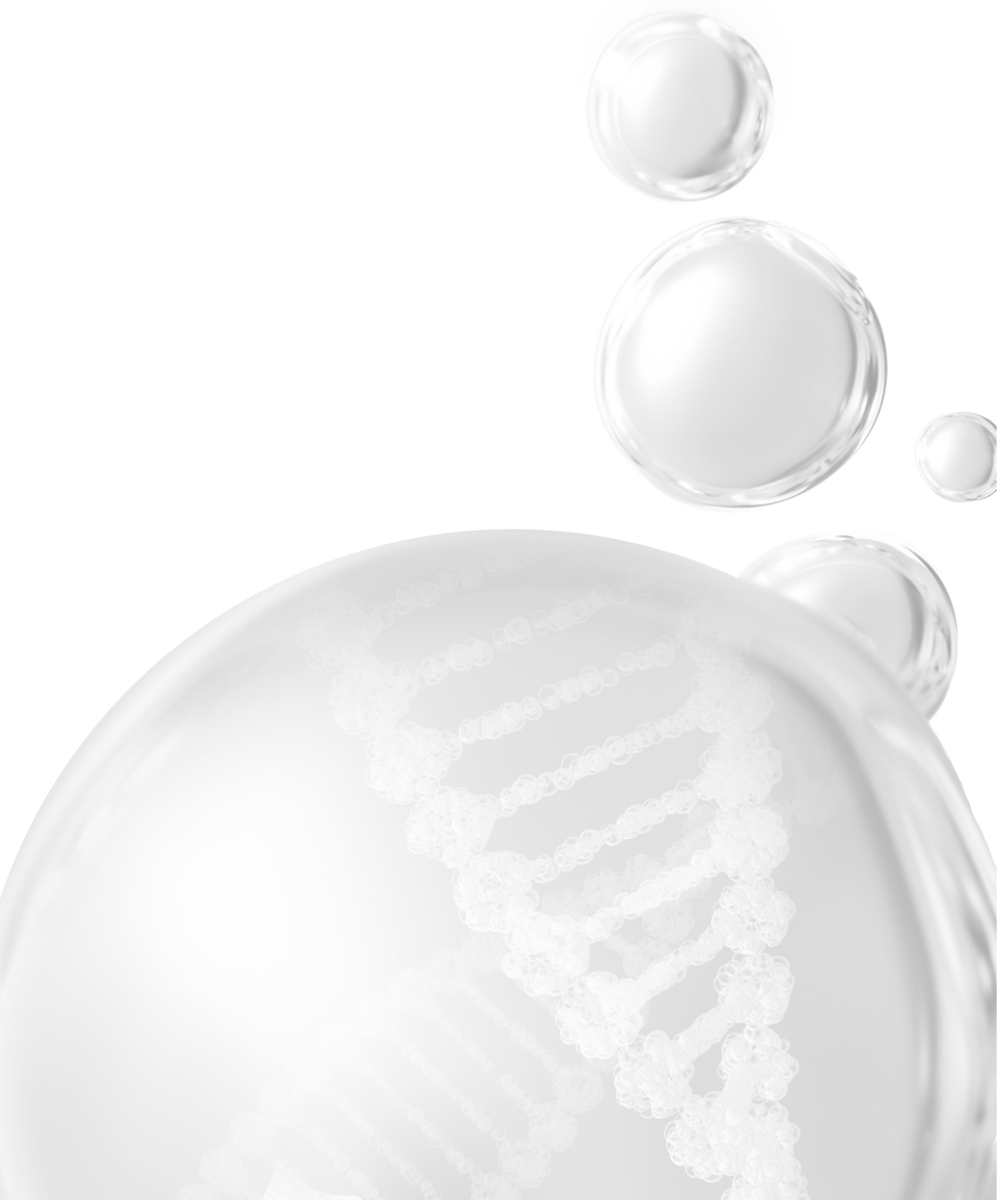


\section{Chapter 7}

\section{ProRaDS: Prostatectomy versus external beam Radiotherapy Decision Support system}

Authors:

Yvonka van Wijk, Bram Ramaekers, Ben Vanneste, Iva Halilaj, Cary Oberije, Avishek Chatterjee, Tom Marcelissen, Arthur Jochems, Henry Woodruff, Philippe Lambin

Adapted from:

"Modeling-based decision support system for radical prostatectomy versus external beam radiotherapy for prostate cancer incorporating an in silico clinical trial and a cost-utility study." Cancers (Basel), 2021 May 29;13(11):2687.

doi: 10.3390/cancers13112687 


\section{Abstract}

Background: Studies have found that survival after radical prostatectomy (RP) and external beam radiotherapy (EBRT) as primary treatment for prostate cancer (PCa) is similar, but the risks of toxicities are not. The aim of this study was to build a decision support system (DSS) using a decision-analytic model (individual statetransition model) based on predictive models (TRIPOD 2-4) for estimating tumor control and toxicity probabilities for both RP and EBRT for low to intermediate risk localized PCa.

Methods: We performed analyses on a synthetically generated dataset of 1000 patients with realistic clinical parameters, externally validated our approach by comparing our results with those from randomized clinical trials, and subsequently set up an in silico clinical trial for elderly patients. We assessed the cost-effectiveness (CE) of the DSS for treatment selection by comparing it to randomized treatment allotment as a proxy for current clinical practice.

Results: Using the DSS to decide upon treatment (RP or EBRT) for the synthetic dataset, $47.8 \%$ were selected for RP and $52.2 \%$ for EBRT. Upon comparison to published results, differences with the simulations of late toxicity and biochemical failure never exceeded $2 \%$. The in silico trial showed that for elderly patients, toxicity has more influence on the decision than TCP, and the predicted QoL depends on the initial erectile function. The DSS is estimated to result in cost savings (€323 (95\% Cl €213-€433)) and more quality adjusted life years (QALYs; 0.11 years, Cl: 0.00-0.22) than randomized treatment selection.

Conclusions: This modelling study shows that DSS based treatment decisions will result in a clinically relevant increase in the patients' quality of life and could be used for in silico trials. 


\section{Introduction}

Prostate cancer (PCa) is the second most commonly diagnosed cancer for men, ac-counting for $13.5 \%$ of new cancers diagnosed in 2018 in the Netherlands, of which $40 \%$ are low- to intermediate-risk localized PCa ${ }^{[1,2]}$. PCa is a topic of heightened research interest, with new biomarkers and treatment modalities being tested at a high rate ${ }^{[3]}$. The leading choices for managing clinically localized PCa are external beam radiotherapy (EBRT), radical prostatectomy (RP), brachytherapy, and active surveillance ${ }^{[4]}$. For low- and intermediate-risk PCa, active surveillance is often proposed (in $\sim 70 \%$ and $\sim 30 \%$ of cases, respectively), and of the active treatment options, RP and EBRT are recommended most often ( $50 \%$ and $\sim 45 \%$ ), according to the Netherlands Cancer Registry. However, no consensus has been reached as to which is superior in terms of effectiveness and/or toxicity, both due to the varying spectrum of toxicities as well as the difference in incidence. The treatment decision is often based on doctor preference and, to a much lesser extent, on patient preferences and patient-specific characteristics or expected outcomes such as (biochemical recurrence-free) survival or toxicity ${ }^{[5]}$. Typically, cost-effectiveness is not taken into account in the treatment decision. Since no calculations are performed based on patient-specific outcomes, one could argue that this treatment selection method is random when considered from an outcome perspective.

Previous work has compared EBRT to RP in terms of long-term survival as well as different toxicities. Chen et al. performed a meta-analysis of the efficacy of EBRT versus $\mathrm{RP}^{[6]}$. They reported no statistical difference in cancer-specific survival for low- and intermediate-risk patients. Potosky et al. ${ }^{[7]}$ and Donovan et al. ${ }^{[8]}$ showed that urinary incontinence and reduced sexual function were more common for RP, but bowel toxicity was more likely after EBRT. Currently, there are no studies available that help assess the individual benefits of EBRT versus RP based on patient characteristics $^{[9-11]}$, even though it has been suggested that parameters such as age, BMI, tumor grade, and pretreatment prostate-specific antigen (PSA) levels do influence both recurrence-free survival and toxicity ${ }^{[12]}$.

The importance of personalized medicine has become progressively evident, and treatment selection for PCa is no exception ${ }^{[13]}$. An important step towards personalized PCa treatment would be a clinical decision support system (DSS), introduced in 2013 ${ }^{[14]}$, that aids in the decision between RP and EBRT. Additionally, when considering the limited resources available for cancer care, it is becoming increasingly important to consider the cost-benefit ratio when comparing treatments to guide the decision-making process ${ }^{[15]}$. The integration of a clinical DSS could aid in this, as it has already for proton therapy ${ }^{[16]}$.

In addition, the possibility of very cost-effective in silico trials (individualized computer simulations used in the development of drugs, devices, or interventions) 
promise to improve clinical research through better design, more transparent and detailed information about possible results, and greater explanatory power in interpreting side effects, as well facilitating the exploration of interactions with the individuals' biology and the long-term or rare effects.

Our hypothesis is that the DSS can accurately replicate the results from published studies and can be used for in silico trials. We also hypothesize that the use of a DSS for treatment selection results in better tumor control, less toxicity, increased patient quality of life (QoL), and improved cost-benefit ratio when compared with current clinical practice based on tumor boards or medical specialist opinion.

The aim of this study is to build such a DSS using predictive models for estimating tumor control and toxicity probabilities for both RP and EBRT for lowto intermediate-risk localized PCa patients and validate this by comparing it to published clinical trials. We also set up an in silico trial using this model-based approach to assess the outcome for elderly patients. Additionally, we compared the cost-effectiveness (CE) of applying this DSS to random treatment decisions as a proxy for current clinical practice (Figure 7.1). The DSS will be made available at www.ai4cancer.ai.

\section{Materials and Methods}

\section{Decision support system}

Markov model

The target population consisted of overall tumor stage T1-T2 PCa patients who were eligible for active treatment (i.e., EBRT and RP). The DSS was developed by constructing an individual state-transition model to estimate the effects and associated costs of treatment with RP vs. EBRT for each patient. Based on patientspecific parameters (e.g., age) and treatment type (EBRT or RP), probabilities of developing long-term toxicities, including rectal bleeding, urinary incontinence, and impotence or a combination, are calculated. After treatment, patients have a risk of progressing to the recurrence state, which is de-pendent on patientspecific parameters (e.g., Gleason score), after which they can develop metastatic disease and subsequently progress to PCa-related death. Furthermore, from any health state, it is possible to die of causes unrelated to cancer (Figure 7.1). The DSS then pro-vides a comparison of tumor control probability (TCP), probability of chronic erectile dysfunction (ED), chronic urinary incontinence (UI), and late rectal bleeding (RB), as well as a comparison of expected costs and quality-adjusted life years (QALYS). Detailed explanation of the transition probabilities are shown in Supplementary Information S7.1. 


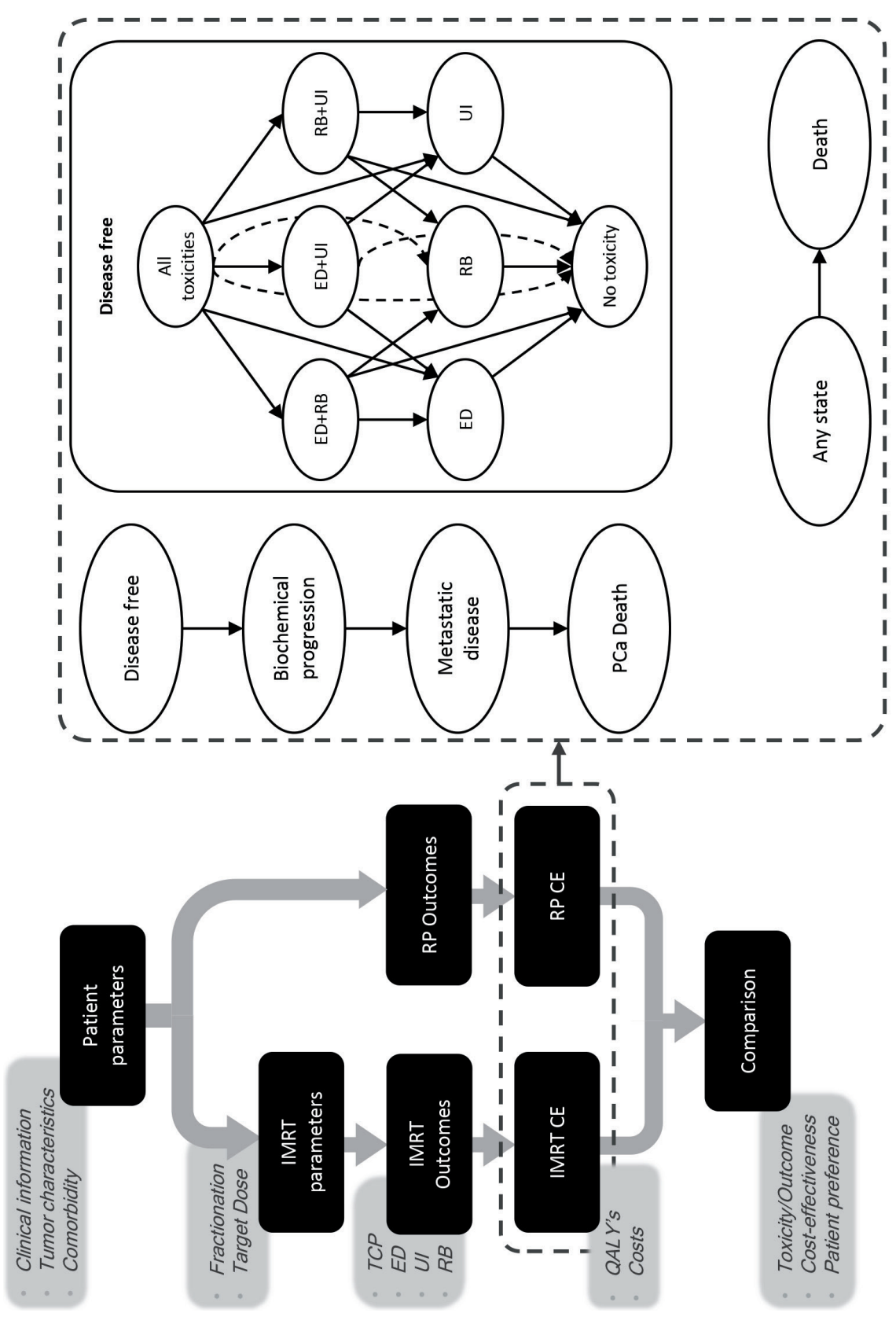

Figure 7.1. Setup of the clinical decision support system and a summary of the Markov model. Ovals represent different health states, arrows represent transitions between health states. IMRT: intensity modulated radiotherapy, RP: radical prostatectomy, CE: costeffectivenesS, QALY: quality adjusted life year, TCP: tumor control probability, ED: erectile dysfunction, UI: urinary incontinence, RB: rectal bleeding, PCa: prostate cancer 
Several assumptions are made in this model, the four most relevant being: (i) All RP patients start with ED and UI (at the first cycle); (ii) when a patient has developed bio-chemical failure, the utility value is described by a single number rather than separate disutility for ED, UI, and RB; (iii) we assume that after progression, the patient cannot re-turn to the cancer-free health state; (iv) the transition probability from recurrence to meta-static disease is the same for all patients for both treatments. Moreover, a cycle time of one month and a time horizon of 20 years were used; these were chosen since the survival of lowintermediate prostate cancer is high, while increasing the time horizon beyond 20 years would unnecessarily increase model uncertainty.

In order to quantify the relative importance of various health outcomes using a common measurement unit, utility is used as a metric to assign weights to health states on a scale ranging from 0 (for dead) to 1.0 (for perfect health). Health-statespecific utilities, also incorporating treatment-related toxicities (and all possible combinations), were retrieved from Stewart et al. ${ }^{[17]}$. QALYs are obtained by multiplying these utility values by the time spent in the corresponding health state.

In order to account for the baseline utility of men living in the Netherlands, we used a published model that had calculated the age-dependent health-related quality of life (HRQoL) for different countries and applied it as a multiplicative factor to health-state-specific utilities ${ }^{[18]}$.

The costs were calculated from the perspective of the healthcare system, so other societal costs, such as productivity losses and patient and family costs, were excluded. For detailed descriptions of the utility and cost data, see Supplementary Information S7.2.

Predictive models

The transition probabilities were estimated per individual in order to make this DSS patient-specific and ready for precision medicine applications. The individual probabilities of progression after treatment and the risk of developing toxicities were calculated using a selection of regression models or nomograms from the published literature (Table 7.1), adherent to the TRIPOD statement ${ }^{[19]}$. For nomograms, the coefficients or intercepts were derived (if not reported) by reading the nomogram and using interpolation and fitting. 
Table 7.1 An overview of the literature models used for the state transition probabilities. Rectal bleeding does not typically occur after RP, so the transition was set to zero for this treatment type.

\begin{tabular}{|c|c|c|c|c|c|}
\hline Model & TRIPOD & Treatment (n) & Parameters & Outcome & Performance \\
\hline $\begin{array}{l}\text { Warner } \\
2015 *[20]\end{array}$ & $\begin{array}{l}1 a \\
+4\end{array}$ & $\begin{array}{l}\text { EBRT } \\
(822+967)\end{array}$ & $\begin{array}{l}\text { ADT [months] } \\
\text { PSA [ng/ml] } \\
\text { Gleason score } \\
\text { BED [Gy] }\end{array}$ & 5-year BFFS & $R^{2}=0.868$ \\
\hline $\begin{array}{l}\text { Bjartell } \\
2016^{[21]}\end{array}$ & 3 & $\begin{array}{l}\text { RP } \\
(3452+1762)\end{array}$ & $\begin{array}{l}\text { T-stage }[\mathrm{T} 1 / \mathrm{T} 2] \\
\text { PSA [ng/ml] } \\
\text { Primary Gleason grade } \\
\text { Secondary Gleason grade } \\
\text { Positive biopsy cores [n] } \\
\text { Negative biopsy cores [n] }\end{array}$ & $\begin{array}{l}\text { 5-year } \\
\text { RFS** }\end{array}$ & $\begin{array}{l}\text { C-index = } \\
0.68\end{array}$ \\
\hline $\begin{array}{l}\text { Schaake } \\
2018^{[22]}\end{array}$ & $1 b$ & $\begin{array}{l}\text { EBRT } \\
(243)\end{array}$ & Mean Trigone dose [Gy] & $\begin{array}{l}\text { Late } \mathrm{UI} \text { after } \\
3 \text { years }\end{array}$ & $A \cup C=0.66$ \\
\hline $\begin{array}{l}\text { Matsushita } \\
2015^{[23]}\end{array}$ & $2 a$ & $\begin{array}{l}\text { RP } \\
(2849)\end{array}$ & $\begin{array}{l}\text { Age [years] } \\
\text { BMI [kg/m2] } \\
\text { ASA score [I/II / III/IV] } \\
\text { Urethral length [mm] }\end{array}$ & $\begin{array}{l}\text { Recovery } \\
\text { from UI } \\
\text { after } 1 \text { year }\end{array}$ & $A \cup C=0.71$ \\
\hline $\begin{array}{l}\text { Alemozaffar } \\
2011^{[24]}\end{array}$ & 3 & $\begin{array}{l}\text { Both } \\
(524+241)\end{array}$ & $\begin{array}{l}\text { Age [years] } \\
\text { Nerve-sparing }[\mathrm{y} / \mathrm{n}] \\
\text { PSA [ng/ml] } \\
\text { ADT }[\mathrm{y} / \mathrm{n}]\end{array}$ & $\begin{array}{l}\text { Erection } \\
\text { recovery } \\
\text { after } 2 \text { years }\end{array}$ & $\begin{array}{l}A \cup C=0.77 \\
\text { for } R P, \\
A \cup C=0.87 \\
\text { for } E B R T\end{array}$ \\
\hline Liu $2010^{[25]}$ & $2 a$ & $\begin{array}{l}\text { EBRT } \\
(161)\end{array}$ & $\begin{array}{l}\text { DVH } \\
\text { V75 }\end{array}$ & $\begin{array}{l}\text { Late RB } \\
\text { after } 3 \text { years }\end{array}$ & $A \cup C=0.62$ \\
\hline
\end{tabular}

TRIPOD: Transparent reporting of a multivariable prediction model for individual prognosis or diagnosis; EBRT: external beam radiotherapy; RP: radical prostatectomy; $n$ : number; ADT: androgen deprivation therapy; PSA: prostate specific antigen; BED: biologically effective dose; BFFS: biochemical failure-free survival; R2: coefficient of determination; Gy: gray; BMI: body mass index; C-index: concordance statistic; RFS: regression free survival; UI: urinary incontinence; ASA: American Society of Anesthesiologists; V75: volume receiving at least 75 Gy; RB: rectal bleeding *Due to incomplete information, some model coefficients had to be derived from the nomogram.

**Recurrence was defined as biochemical failure, initiation of secondary therapy, distant metastases or prostate cancer related death 


\section{Validation}

Synthetic dataset

Since no complete patient cohort for which all required input parameters were known was identified, we generated a synthetic patient dataset. A patient is described by a set of parameters, which we randomly assigned by drawing values from a distribution. We chose the distributions based on the patient cohorts on which the models had been developed (Supplementary Table S7.9). We assumed that the clinical parameters were independent of one another and generated a synthetic patient dataset including 1000 patients so that the mean and associated error of the generated clinical parameters matched that of the original datasets.

\section{Validation of NTCP and TCP models}

In order to validate the combination of our NTCP and TCP models, we compared the simulation results to the published results. The reliability of the model and the measure in which the synthetic dataset reflects real patient datasets were assessed by generating the synthetic patients so that they matched the reported clinical parameters from actual trials, such as age, Gleason score, PSA values, and T-stage. Nonreported clinical parameters were kept the same as the original synthetic dataset. Dosimetric parameters were scaled according to the 2 Gy equivalent dose. The relevant outcomes, such as biochemical free survival or toxicity, were then compared between the simulation and the clinical trial. For the EBRT $\alpha / \beta$ ratio (a measure of the fractionation sensitivity of the tissue) of $\mathrm{PCa}$, we used a value of $1.5 \mathrm{~Gy}^{[26]}$.

In order to assess the credibility of the predicted biochemical free survival, the model was compared to the results of a randomized clinical trial, reported by Hamdy (2016), that compared EBRT to $\mathrm{RP}^{[27]}$; to assess the effect of hypofractionation, we compared bio-chemical free survival to the $\mathrm{CHHiP}$ tria|[28]. In order to validate the model's predictions of toxicity, we compared the model results to Donovan et al. (2016), who published patient-reported outcomes after EBRT or $\mathrm{RT}^{[8]}$.

\section{In silico trial}

A DSS such as this, in combination with the synthetic patient dataset, could function as an in silico clinical trial, a precursor to actual clinical trials, in order to improve study design or explanatory power. We demonstrated this by generating a patient dataset with patients aged 75-80 to test the outcomes of different treatments for elderly patients, an of-ten-underrepresented group in clinical trials, but a group that might still be eligible for both treatments. We adjusted pretreatment erectile function to an average of $15 \%$ to reduce the impact of ED on the outcome. 
Cost-effectiveness analysis

In order to determine the potential benefit of a DSS for PCa, we performed CE anal-yses that compared two different treatment allotment strategies. In the first, each patient in the synthetic dataset was allotted the treatment for which the DSS calculated the highest number of QALYs. For the second, each patient was allotted a randomized treatment as a proxy for current clinical practice.

To determine which treatment allotment strategy was cost-effective, the incremental net monetary benefit (iNMB) was calculated. This is described by the following Equation 7.1:

$$
i N M B=\left(Q A L Y_{D S S}-Q A L Y_{\text {random }}\right) \cdot W T P-\left(\text { Costs }_{D S S}-\text { Costs }_{\text {random }}\right)
$$

${\text { Where } \text { Costs }_{\text {DSS }} \text { and Costs }}_{\text {random }}$ are the mean costs per patient if treatment decisions are based on a DSS or randomized treatment allotment strategy respectively, and $Q A L Y_{D S S}$ and $Q A L Y_{\text {random }}$ are the mean number of QALYS per patient. WTP is the willingness to pay per QALY. If the iNMB is positive, the DSS was considered cost-effective. In the Netherlands, a WTP threshold of $€ 80,000$ is agreed upon for high burden diseases ${ }^{[29]}$. We applied discount rates of $1.5 \%$ for the QALYs, and $4 \%$ for the costs, which is standard practice within the Netherlands ${ }^{[30]}$.

\section{Results}

\section{Synthetic data characteristics}

When applying the DSS on a synthetically generated patient dataset of 1000 patients with clinical parameters similar to those on which the predictive models were built, $47.8 \%$ of the patients had a higher number of QALYs for RP and $52.2 \%$ for EBRT. The patients for whom EBRT was chosen had a higher mean age (64 versus 59 years), higher mean prostate specific antigen (PSA) values (8.3 versus 6.8 $\mathrm{ng} / \mathrm{ml}$ ), and a higher percentage of T2 stage (49\% versus $24 \%$ ), as shown in Table 7.2 and Supplementary Table S7.10. 
Table 7.2 Patient characteristics of the synthetic patient dataset

\begin{tabular}{l|lll} 
Parameter name & EBRT mean (SD) & RP mean (SD) & $\boldsymbol{P}$ \\
\hline Age (years) & $63.8(10.7)$ & $58.8(9.1)$ & $<<0.001$ \\
PSA (ng/mL) & $8.3(3.5)$ & $6.8(3.5)$ & $<<0.001$ \\
T-stage 2 (\%) & 48.7 & 24.4 & $<<0.001$ \\
P. Gleason grade 4 (\%) & 27.1 & 3.3 & $<<0.001$ \\
S. Gleason grade 4 (\%) & 14.7 & 20.9 & 0.01 \\
ADT given (\%) & 79.8 & 0 & $<<0.001$ \\
\hline
\end{tabular}

PSA: prostate specific antigen; T-stage: tumor stage; P.: primary; S.: secondary; ADT: androgen deprivation therapy

\section{Validation}

We compared the outcome reported in three separate papers against the same out-come simulated by our DSS on a synthetic patient dataset; the results are reported in Table 7.3. More details on the parameter values used are shown in Supplementary Table S7.11, and results are shown in Figure 7.2. The progressionfree survival reported by Hamdy (2016) was very similar to our simulation results, as was the Kaplan-Meier curves obtained from the GUROC (Genitourinary Radiation Oncologists of Canada) ${ }^{[31]}$ Prostate Cancer Risk Stratification (ProCaRS) database ${ }^{[32]}$, and the National Prostate Cancer Register of Sweden (NPCR) ${ }^{[21,33]}$. Dearnaley (2016) published the results of the $\mathrm{CHHiP}$ trial and compared the results of different fractionation plans ${ }^{[28]}$. When comparing these results to what was simulated by our DSS, the simulation was close to the published results, but the difference increased with stronger hypofractionation. Donovan (2016) was used for toxicity comparisons and showed that the DSS simulated late toxicity best but was less accurate for acute toxicity. The relative differences between the simulations and the studies for the different treatment modalities were similar, and the conclusions coincided. The most notable discrepancy is for acute ED for EBRT, which was underestimated by the simulations because we assumed no acute ED after EBRT treatment. 

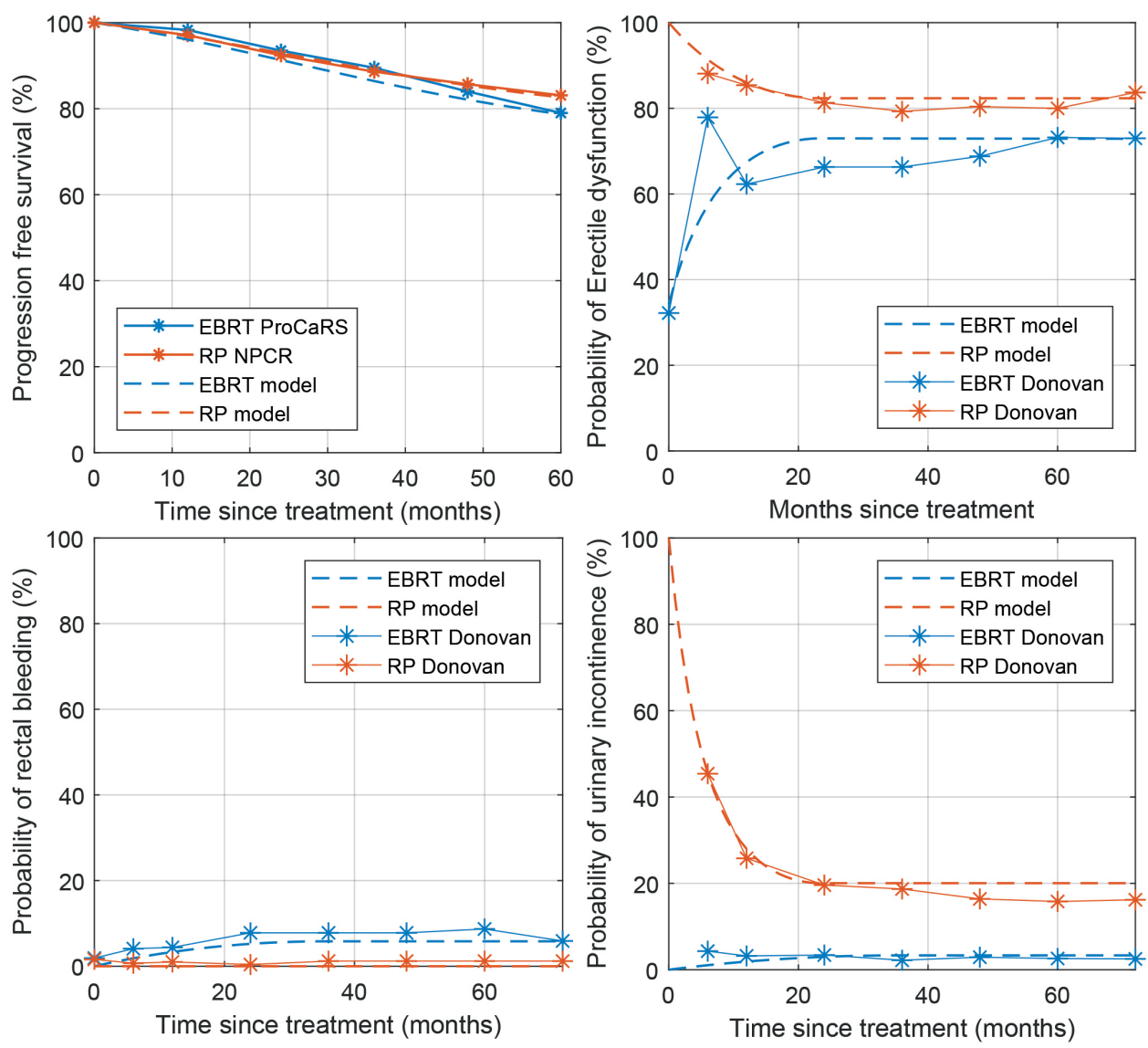

Figure 7.2. Progression free survival and probability of toxicity of the model compared to published data. Progression free survival data was obtained from the GUROC (Genitourinary Radiation Oncologists of Canada) Prostate Cancer Risk Stratification (ProCaRS) database, and the National Prostate Cancer Register of Sweden (NPCR). Data on toxicity was obtained from Donovan 2016 et al.

\section{In silico trial}

We performed an in silico trial on the synthetic dataset by increasing the age of all patients to between 75 and 80 but leaving all other clinical parameters as they were. We found that TCP for RP was marginally higher than for EBRT (HR: 1.007), and the risk of chronic UI was much higher for RP (HR: 10) as well as ED (HR: 1.59); consequently, EBRT did result in more QALYs and lower costs than RP. The DSS selected EBRT for $96 \%$ of the patients. We repeated this analysis while assuming that $85 \%$ of the patients had ED before the start of treatment. This resulted in a smaller difference in ED, thus decreasing the dif-ference in QALYs between RP and EBRT (Table 7.4) and resulting in the DSS selecting EBRT for $72 \%$ of the patients. 
Table 7.3 Patient characteristics of the synthetic patient dataset

\begin{tabular}{|c|c|c|c|c|c|}
\hline Study & Treatment [N] & Outcome & $\begin{array}{l}\text { Published } \\
\text { study (\%) }\end{array}$ & Simulation (\%) & Difference (\%) \\
\hline \multirow[t]{2}{*}{ Hamdy 2016} & EBRT [545] & 5 year BDFS & 88.5 & 89.0 & 0.5 \\
\hline & RP [553] & 5 year BDFS & 88.1 & 87.0 & 1.1 \\
\hline \multirow[t]{3}{*}{ CHHiP } & $\begin{array}{l}\text { EBRT } 74 \text { Gy } \\
{[1065]}\end{array}$ & 5 year BDFS & 88.3 & 88.4 & 0.1 \\
\hline & $\begin{array}{l}\text { EBRT } 60 \text { Gy } \\
{[1074]}\end{array}$ & 5 year BDFS & 90.6 & 89.9 & 0.7 \\
\hline & $\begin{array}{l}\text { EBRT } 57 \text { Gy } \\
{[1077]}\end{array}$ & 5 year BDFS & 85.9 & 87.9 & 2.0 \\
\hline \multirow[t]{2}{*}{$\begin{array}{l}\text { Donovan } \\
2016\end{array}$} & EBRT [545] & $\begin{array}{l}6 \text { month UI } \\
6 \text { year UI } \\
6 \text { month ED } \\
6 \text { year ED } \\
6 \text { month RB } \\
6 \text { year RB }\end{array}$ & \begin{tabular}{l|}
5 \\
3.5 \\
77.8 \\
73.0 \\
3.8 \\
5.9
\end{tabular} & \begin{tabular}{l|l}
3.3 \\
3.3 \\
57.3 \\
72.9 \\
1.9 \\
5.8
\end{tabular} & $\begin{array}{l}1.7 \\
0.2 \\
20.5 \\
0.1 \\
1.9 \\
0.1\end{array}$ \\
\hline & RP [553] & $\begin{array}{l}6 \text { month UI } \\
6 \text { year UI } \\
6 \text { month ED } \\
6 \text { year ED }\end{array}$ & $\begin{array}{l}46 \\
17 \\
88 \\
83.5\end{array}$ & $\begin{array}{l}45.5 \\
20.1 \\
91.3 \\
82.4\end{array}$ & $\begin{array}{l}0.5 \\
3.1 \\
3.3 \\
1.1\end{array}$ \\
\hline
\end{tabular}

$N$ : number of patients; EBRT: external beam radiotherapy; RP: radical prostatectomy; BDFS: biological disease-free survival; UI: urinary incontinence; ED: erectile dysfunction; RB: rectal bleeding; Gy: gray; GS: Gleason score

Table 7.4 In Silico trial results

\begin{tabular}{|c|c|c|c|c|c|c|}
\hline Outcome type & $\begin{array}{l}R P \text { mean } \\
\text { (SD) }\end{array}$ & $\begin{array}{l}\text { EBRT } \\
\text { mean (SD) }\end{array}$ & $\begin{array}{l}\text { DSS mean } \\
\text { (SD) }\end{array}$ & $\begin{array}{l}\text { RP PTED } \\
\text { mean (SD) }\end{array}$ & $\begin{array}{l}\text { EBRT PTED } \\
\text { mean (SD) }\end{array}$ & $\begin{array}{l}\text { DSS PTED } \\
\text { mean (SD) }\end{array}$ \\
\hline 5 year TCP $[\%]$ & $84(7)$ & $84(4)$ & $84(4)$ & $84(7)$ & $84(4)$ & $85(5)$ \\
\hline 2 year ED [\%] & $93(6)$ & $61(19)$ & $61(20)$ & $100(0)$ & $98(2)$ & $98(2)$ \\
\hline 1 year UI [\%] & $70(16)$ & $7(4)$ & $8(10)$ & $70(16)$ & $7(4)$ & $22(26)$ \\
\hline 3 year $R B[\%]$ & $0(0)$ & $5(4)$ & $5(4)$ & $0(0)$ & $5(4)$ & $3(4)$ \\
\hline QALY & $\begin{array}{l}4.28 \\
(0.87)\end{array}$ & $\begin{array}{l}4.46 \\
(0.86)\end{array}$ & $\begin{array}{l}4.46 \\
(0.86)\end{array}$ & $\begin{array}{l}4.25 \\
(0.85)\end{array}$ & $4.30(0.83)$ & $4.32(0.84)$ \\
\hline Costs (1000 €) & $15.2(1.1)$ & $12.6(1.0)$ & $12.7(1.1)$ & $15.2(1.1)$ & $12.7(1.0)$ & $13.3(1.5)$ \\
\hline
\end{tabular}

RP: Radical prostatectomy; EBRT: external beam radiotherapy; DSS: decision support system; PTED: 85\% pretreatment erectile dysfunction; SD: 1 standard deviation; TCP: tumor control probability; ED: Erectile dysfunction; UI: Urinary incontinence; RB: rectal bleeding QALY: quality adjusted life years

Cost-effectiveness analysis

When comparing the DSS treatment selection to a randomized treatment allotment strategy, the DSS resulted in an average cost saving (discounted) of EUR 317 per patient and an increase in the number of discounted QALYS of 0.11 years. 
With an incidence rate of 12,500 patients a year in the Netherlands, with $40 \%$ being low- to intermediate-risk PCa patients and 50\% receiving active treatment, this DSS could affect 2400 patients a year. Since the costs decreased while the QALYs increased, the DSS was the dominant strategy in this "base case" scenario, with an iNMB (Equation (7.1)) of EUR 8798 per patient. The TCP is higher for the DSS than for randomized treatment allotment, and the probability of all toxicities decreases with the use of this DSS (Table 7.5).

Table 7.5 Probability of different outcomes

\begin{tabular}{|c|c|c|c|c|c|c|}
\hline Outcome type & $R P$ mean (SD) & $\begin{array}{l}E B R T \\
\text { mean (SD) }\end{array}$ & $P$ & $\begin{array}{l}\text { Random } \\
\text { mean (SD) }\end{array}$ & DSS mean (SD) & $P$ \\
\hline 5 year TCP $[\%]$ & $83(8)$ & $84(4)$ & 0.003 & $83(7)$ & $85(5)$ & $<<0.001$ \\
\hline $\begin{array}{l}2 \text { year Erectile } \\
\text { dysfunction [\%] }\end{array}$ & $83(13)$ & $59(2)$ & $<<0.001$ & $71(14)$ & $67(20)$ & $<<0.001$ \\
\hline $\begin{array}{l}1 \text { year Urinary } \\
\text { incontinence [\%] }\end{array}$ & $27(18)$ & $7(4)$ & $<<0.001$ & $16(9)$ & $13(15)$ & $<<0.001$ \\
\hline $\begin{array}{l}3 \text { year Rectal } \\
\text { bleeding [\%] }\end{array}$ & $0(0)$ & $5(4)$ & $<<0.001$ & $2(2)$ & $1(3)$ & 0.61 \\
\hline
\end{tabular}

RP: Radical prostatectomy; EBRT: external beam radiotherapy; DSS: decision support system; SD: standard deviation; TCP: tumor control probability 


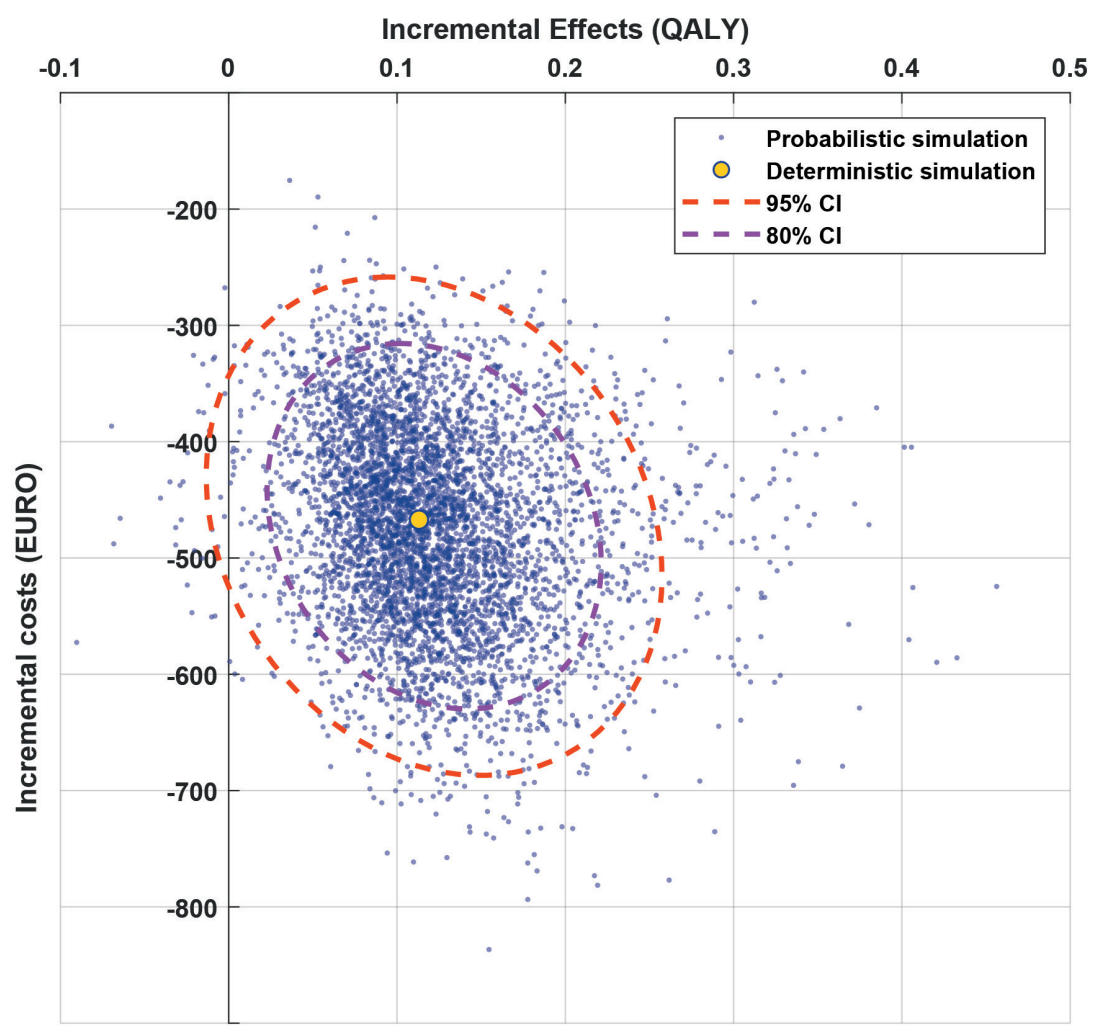

Figure 7.3. The cost-effectiveness plane when comparing the DSS to the randomized strategy. Each blue point represents the incremental costs and quality adjusted life years (QALYS) for DSS vs randomized treatment allocation of a single simulation over all 1000 patients. The yellow dot shows the deterministic simulation, and the dashed lines show confidence intervals of the simulations.

A probabilistic sensitivity analysis, performed with 5,000 Monte-Carlo simulations, resulted in a cohort with a mean increase in discounted QALYs of 0.11 (95\% Cl 0.00-0.22), and a mean decrease in costs of €323 (95\% Cl €213-€433) (Figure 3) for DSS compared with randomized treatment allocation. For $98.6 \%$ of the simulations, using a DSS was cost-effective when compared to a randomized strategy (dominant for $98.2 \%$ of the simulations). For a detailed cost-effectiveness analysis of the model, see Supplementary Information S7.3.

\section{Discussion}

In this study, we developed a clinical DSS for the treatment of PCa patients with either EBRT or RP and tested this on a synthetic patient dataset. We validated the DSS against published clinical studies and set up an in silico trial for patients 
between 75 and 80, eligible for both RP and EBRT. We also assessed the CE of a treatment allotment strategy based on the DSS compared to a randomized treatment allotment strategy. Our first hypothesis was that we could accurately replicate results from published studies, which we aimed to confirm by generating synthetic datasets with clinical parameters similar to published trials. The DSS largely replicated the published results accurately. The relative differences between the treatment modalities and fractionation plans were replicated by the model, and the conclusions of the DSS and the studies agreed. We also performed an in silico trial, exclusively including elderly patients first without, then with, prior ED, using the DSS and found that for the first group, EBRT was preferred, and, for the second, RP performed better in terms of QALYs. Additionally, we hypothesized that a treatment se-lection strategy based on the DSS would improve tumor control, reduce toxicity, and im-prove CE as opposed to randomized treatment selection. Our CE analyses suggest that not only do the costs of treatment decrease with the application of a DSS, but the number of QALYS also increases, making the integration of a DSS dominantly cost-effective com-pared to current clinical practice. The expected cost savings within the Netherlands when using a DSS could be as high as EUR 3.8 million lover five years, assuming 2400 patients are affected every year. Additionally, the number of patients with recurrence after treatment could be reduced slightly by $2 \%$.

These results imply that when deciding between RP and EBRT for a given patient, making the right choice can improve overall QoL and that this decision should not be random. The DSS offers the possibility of combining a large number of clinical parameters to predict NTCP and TCP and quantify these risks into a single metric for different treatment options. This has the potential to improve the decision-making process, along with other factors such as incorporating patient preferences. The development of a DSS fits well into the current trend that strives for personalized medicine, and the results presented in this study confirm the added benefit of such a too ${ }^{[34]}$. The application of the DSS for in silico trials has great potential benefits; it not only improves the design of clinical trials through precursory simulations but also has the benefit of being able to apply different treatments to the same "patient", which allows for a more objective comparison. Another advantage of the DSS is that it is detailed and can be further extended with other disease management options such as brachytherapy or active surveillance. The framework of the DSS is also flexible, making it easy to replace individual models or update them as new clinical trial results are published. It can also be used as the basis for an individualized patient decision aid (iPDA).

This study has several limitations. The first one is that this is a model-based study, using models that were trained and validated on different cohorts. The models were selected based on how recently they were published, the number of 
patients included, and whether or not they used clinical parameters and a TRIPOD level. We also attempted to make sure we only selected models trained on patients with similar treatment modalities and similar clinical parameters; however, not all clinical parameters were reported. In addition, the correlation between clinical parameters was not reported, and when generating the synthetic dataset, no correlations were assumed. It should also be noted that the clinical parameters found in the clinical trials used as a basis for the synthetic dataset may not be representative of the whole patient population. Different outcomes of the models were validated in different studies, so the DSS as a whole has not been validated on a single patient population. However, the acquisition of a dataset where not only all the clinical parameters but also long-term follow-up data for TCP and toxicity for both treatment arms are reported might not be feasible. Moreover, the NTCP models used doctor-reported out-comes as endpoints, while the validation was done on patient-reported outcomes. How-ever, the promising benchmark results indicate that the models have significant value in simulating reality. These results were generated using an $\alpha / \beta$ ratio of 1.5 ; should this value be proven inaccurate, these results will have to be reassessed.

In the CE analysis, we used randomized treatment allotment as a proxy for clinical practice; however, we were not able to compare the performance of the CE model to actual clinical practice. This is because there is no hard baseline and actual clinical practice can vary strongly between countries and even hospitals. However, in Supplementary Information S7.3, we do compare the performance of the CE model to simple clinical parameters, which, in clinical practice, could help determine treatment choice. In its current state, the DSS does not take into account patient preferences but uses average utility values obtained from a population. However, the risks of different types of toxicities are what often drive treatment decision-making, and patient preferences should be taken into account. Future versions of the DSS should allow patients' input to affect the utility values of different toxicities and be integrated into iPDAs to allow for shared decisionmaking ${ }^{[35,36]}$. This is especially true for the $15 \%$ of patients for whom the expected difference in QALYs is very low ( $<0.05$ years).

A limitation of the reported CE analysis is that both the DSS and the CE model were based on the same state-transition model (committing a potential petitio principii fallacy). The differences between the two were that the DSS was based on the undiscounted deterministic run, while the CE model used discount rates for both QALYs and costs and was based on probabilistic simulations. The costs used in this study were based on the health costs in the Netherlands. One of the assumptions in the model was that if the patient had biochemical failure after primary treatment, no secondary treatment would take place, though, often, this is not the case. The health-state recurrence now has the same costs as a regular 
follow-up, but with secondary treatment, these costs would be higher. However, when applying the model with higher costs for recurrence, matching them with the costs for metastatic disease, the cost-effectiveness was not impacted, so this assumption has no direct impact on our conclusions. For the application of the tool in other countries, the costs will be most complex to adapt. However, utility values are simpler to adjust to different countries, so it might be possible only to transfer the section of the tool that calculates QALYs, which already provides valuable information that can support decision making. However, it should be aspired to include the monetary calculations, as these play an increasingly large role in healthcare decision making.

Short-term future work includes merging this DSS with previously developed DSSs, including one where the effectiveness of an implantable rectum spacer is accessed on the patient leve ${ }^{[37,38]}$ as well as a DSS that compares photon to proton therapy. We also plan to expand the NTCP models by including single nucleotide polymorphisms and tumor somatic mutations to incorporate genetic information into the decision-making process ${ }^{[39]}$.

\section{Conclusion}

This study lays the groundwork for a detailed, personalized treatment DSS that aids in the choice between EBRT and RP for low to intermediate risk PCa patients. This DSS could be used for in silico clinical trials when applied to a synthetic dataset, which would be a valuable precursor to clinical trials. The results suggest that the full development and clinical application of this DSS would improve the quality of patient care as well as cost-effectiveness and would be an important step towards personalized and participative treatment decisions. 


\section{References}

1. Bray F, Ferlay J, Soerjomataram I, Siegel RL, Torre LA, Jemal A. Global cancer statistics 2018: GLOBOCAN estimates of incidence and mortality worldwide for 36 cancers in 185 countries. CaCancer J Clin 2018; 68(6): 394-424.

2. D’Amico AV, Whittington R, Malkowicz SB, et al. Biochemical outcome after radical prostatectomy, external beam radiation therapy, or interstitial radiation therapy for clinically localized prostate cancer. Jama-J Am Med Assoc 1998; 280(11): 969-74.

3. Filella X, Fernandez-Galan E, Fernandez Bonifacio R, Foj L. Emerging biomarkers in the diagnosis of prostate cancer. Pharmgenomics Pers Med 2018; 11: 83-94.

4. Lane JA, Donovan JL, Davis M, et al. Active monitoring, radical prostatectomy, or radiotherapy for localised prostate cancer: study design and diagnostic and baseline results of the ProtecT randomised phase 3 trial. Lancet Oncology 2014; 15(10): 1109-18.

5. Heidenreich A, Bastian PJ, Bellmunt J, et al. EAU Guidelines on Prostate Cancer. Part 1: Screening, Diagnosis, and Local Treatment with Curative Intent-Update 2013. Eur Urol 2014; 65(1): 124-37.

6. Chen L, Li Q, Wang Y, Zhang Y, Ma X. Comparison on efficacy of radical prostatectomy versus external beam radiotherapy for the treatment of localized prostate cancer. Oncotarget 2017; 8(45): 79854-63.

7. Potosky AL, Davis WW, Hoffman RM, et al. Five-year outcomes after prostatectomy or radiotherapy for prostate cancer: the prostate cancer outcomes study. J Natl Cancer Inst 2004; 96(18): 1358-67.

8. Donovan JL, Hamdy FC, Lane JA, et al. Patient-Reported Outcomes after Monitoring, Surgery, or Radiotherapy for Prostate Cancer. New Engl J Med 2016; 375(15): 1425-37.

9. van Wijk Y, Halilaj I, van Limbergen E, et al. Decision Support Systems in Prostate Cancer Treatment: An Overview. Biomed Res Int 2019; 2019: 4961768.

10. Walsh S, de Jong EEC, van Timmeren JE, et al. Decision Support Systems in Oncology. JCO Clin Cancer Inform 2019; 3: 1-9.

11. Lambin P, Zindler J, Vanneste BG, et al. Decision support systems for personalized and participative radiation oncology. Adv Drug Deliv Rev 2017; 109: 131-53.

12. Shariat SF, Karakiewicz PI, Margulis V, Kattan MW. Inventory of prostate cancer predictive tools. Curr Opin Urol 2008; 18(3): 279-96.

13. Lambin P, Zindler J, Vanneste B, et al. Modern clinical research: How rapid learning health care and cohort multiple randomised clinical trials complement traditional evidence based medicine. Acta Oncol 2015; 54(9): 1289-300.

14. Lambin P, van Stiphout RGPM, Starmans MHW, et al. Predicting outcomes in radiation oncologymultifactorial decision support systems. Nature Reviews Clinical Oncology 2013; 10(1): 27-40.

15. Meropol NJ, Schrag D, Smith TJ, et al. American Society of Clinical Oncology guidance statement: the cost of cancer care. J Clin Oncol 2009; 27(23): 3868-74.

16. Widder J, van der Schaaf A, Lambin P, et al. The Quest for Evidence for Proton Therapy: ModelBased Approach and Precision Medicine. Int J Radiat Oncol Biol Phys 2016; 95(1): 30-6.

17. Stewart ST, Lenert L, Bhatnagar V, Kaplan RM. Utilities for prostate cancer health states in men 
aged 60 and older. Med Care 2005; 43(4): 347-55.

18. Heijink R, van Baal P, Oppe M, Koolman X, Westert G. Decomposing cross-country differences in quality adjusted life expectancy: the impact of value sets. Popul Health Metr 2011; 9(1): 17.

19. Collins GS, Reitsma JB, Altman DG, Moons KGM. Transparent Reporting of a Multivariable Prediction Model for Individual Prognosis or Diagnosis (TRIPOD): the TRIPOD statement. Bjog-Int J Obstet Gy 2015; 122(3): 434-43.

20. Warner A, Pickles T, Crook J, et al. Development of ProCaRS Clinical Nomograms for Biochemical Failure-free Survival Following Either Low-Dose Rate Brachytherapy or Conventionally Fractionated External Beam Radiation Therapy for Localized Prostate Cancer. Cureus 2015; 7(6): e276.

21. Bjartell A, Bottai M, Persson J, et al. Prediction of clinical progression after radical prostatectomy in a nationwide population-based cohort. Scand J Urol 2016; 50(4): 255-9.

22. Schaake W, van der Schaaf A, van Dijk LV, van den Bergh ACM, Langendijk JA. Development of a prediction model for late urinary incontinence, hematuria, pain and voiding frequency among irradiated prostate cancer patients. PLoS One 2018; 13(7): e0197757.

23. Matsushita K, Kent MT, Vickers AJ, et al. Preoperative predictive model of recovery of urinary continence after radical prostatectomy. Bju Int 2015; 116(4): 577-83.

24. Alemozaffar M, Regan MM, Cooperberg MR, et al. Prediction of erectile function following treatment for prostate cancer. JAMA 2011; 306(11): 1205-14.

25. Liu M, Moiseenko V, Agranovich A, et al. Normal Tissue Complication Probability (NTCP) modeling of late rectal bleeding following external beam radiotherapy for prostate cancer: A Test of the QUANTEC-recommended NTCP model. Acta Oncol 2010; 49(7): 1040-4.

26. Miralbell R, Roberts SA, Zubizarreta E, Hendry JH. Dose-fractionation sensitivity of prostate cancer deduced from radiotherapy outcomes of 5,969 patients in seven international institutional datasets: alpha/beta = 1.4 (0.9-2.2) Gy. Int J Radiat Oncol Biol Phys 2012; 82(1): e17-24.

27. Hamdy FC, Donovan JL, Lane JA, et al. 10-Year Outcomes after Monitoring, Surgery, or Radiotherapy for Localized Prostate Cancer. N Engl J Med 2016; 375(15): 1415-24.

28. Dearnaley D, Syndikus I, Mossop H, et al. Conventional versus hypofractionated high-dose intensity-modulated radiotherapy for prostate cancer: 5-year outcomes of the randomised, noninferiority, phase 3 CHHiP trial. Lancet Oncol 2016; 17(8): 1047-60.

29. Care tCfPHaH. Sensible and Sustainable care. Zoetermeer; 2006.

30. Attema AE, Brouwer WBF, Claxton K. Discounting in Economic Evaluations. Pharmacoeconomics 2018; 36(7): 745-58.

31. Hood L, Friend SH. Predictive, personalized, preventive, participatory (P4) cancer medicine. Nat Rev Clin Oncol 2011; 8(3): 184-7.

32. Barry MJ, Edgman-Levitan S. Shared decision making--pinnacle of patient-centered care. N Engl J Med 2012; 366(9): 780-1.

33. Sferrazza S, Accettone R, Pampoorickel K, et al. Men's experiences of deciding about treatment for localized prostate cancer: a meta-synthesis. Prof Inferm 2019; 72(4): 272-82.

34. van Wijk Y, Vanneste BGL, Walsh S, et al. Development of a virtual spacer to support the decision 
for the placement of an implantable rectum spacer for prostate cancer radiotherapy: Comparison of dose, toxicity and cost-effectiveness. Radiotherapy and Oncology 2017; 125(1): 107-12.

35. van Wijk Y, Vanneste BL, Jochems A, et al. Development of an isotoxic decision support system integrating genetic markers of toxicity for the implantation of a rectum spacer. Acta Oncologica 2018; 57(11): 1499-505.

36. Bergom C, West CM, Higginson DS, et al. The Implications of Genetic Testing on Radiation Therapy Decisions: A Guide for Radiation Oncologists. Int J Radiat Oncol 2019; 105(4): 698-712. 


\section{Supplementary Material}

\section{Supplementary Information S7.1: Calculation of transition probabilities}

Within the Markov model used for the decision support system (DSS), the health state "disease free" consists a number of sub-heath states, namely no toxicity, or any combination of three different toxicities: rectal bleeding, urinary incontinence and erectile dysfunction. From the models we have available the probability that a patient has one of three different toxicities after a certain amount of time, however we need to transfer this into transition probabilities within our model. To explain how these were calculated please refer to the diagram in Supplementary Figure S7.1 below.

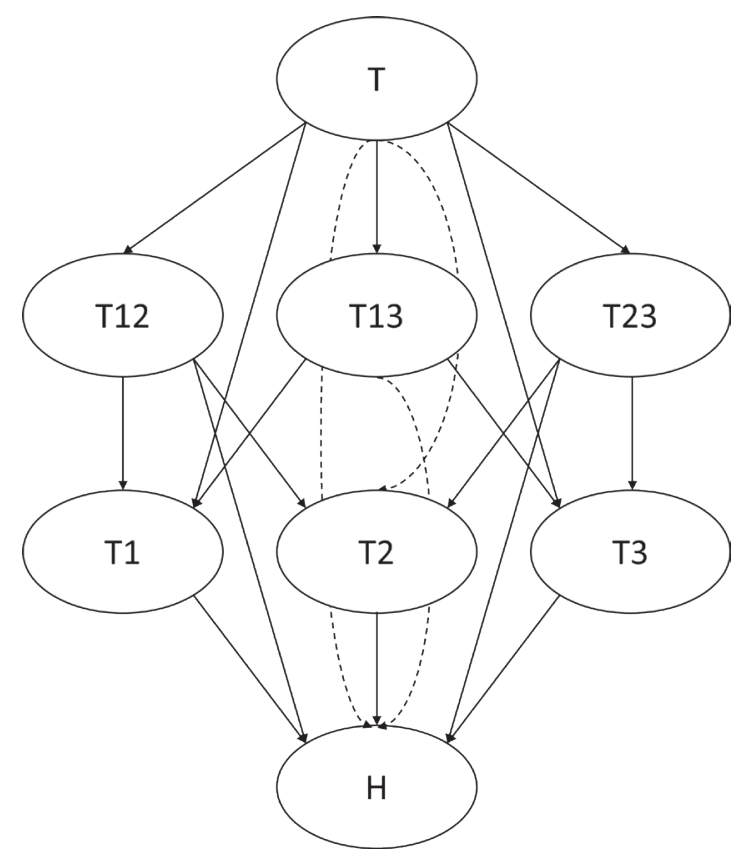

Supplementary Figure S7.1: The transition probability diagram of the "Healthy" life state. $T$ = all three toxicities; $H$ = no toxicities; $T 1$ = erectile dysfunction; $T 2$ = rectal bleeding; $T 3=$ urinary incontinence; $T 12$ = erectile dysfunction and rectal bleeding; $T 13=$ erectile dysfunction and urinary incontinence; T23 = rectal bleeding and urinary incontinence

This diagram represents patients treated with prostatectomy, as they have a probability to recover from toxicity, but they will never get a toxicity if they don't have it initially. We now need to calculate the separate transition probabilities using the probability of a patient recovering from a certain toxicity. First, we convert the probability calculated from the nomograms to the probability per cycle.

P1 = probability of recovering from Erectile dysfunction per cycle

P2 = probability of recovering from Rectal bleeding per cycle

P3 = probability of recovering from Urinary incontinence per cycle 
First, we will calculate the probability of going from all toxicities to any other health state, as shown in Supplementary Figure S7.2 below.

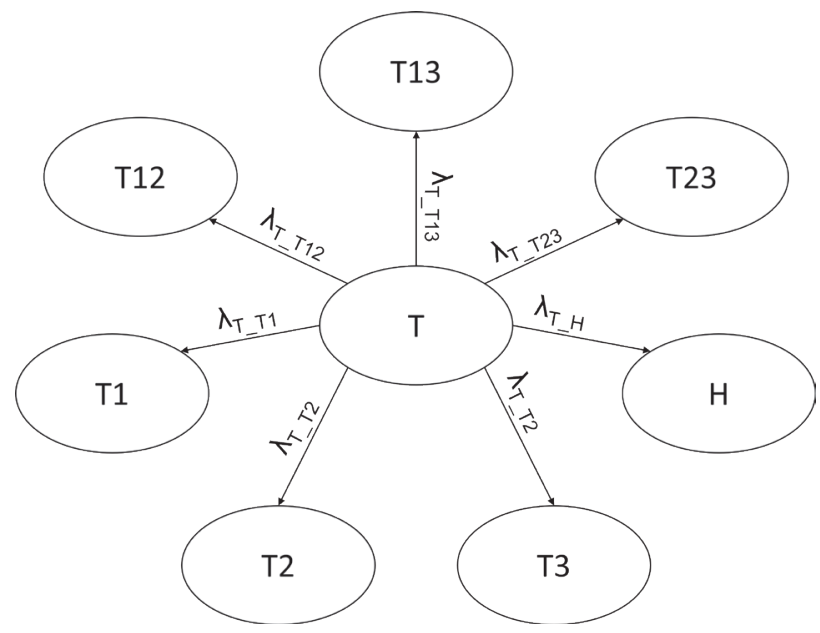

Supplementary Figure 57.2: A diagram showing the transition from all toxicities health state to all other health states

The probability of recovering from all three toxicities in one cycle can be obtained using the following relation:

$$
\lambda_{T \_H}=P 1 * P 2 * P 3
$$

The probability of recovering from two different toxicities can be calculated by multiplying the probability of recovering from either toxicity, subtracted by the probability of recovering from all three toxicities.

$$
\begin{aligned}
& \lambda_{T_{-} T 1}=P 2 * P 3-\lambda_{T_{-} H} \\
& \lambda_{T_{-} T 2}=P 1 * P 3-\lambda_{T_{-} H} \\
& \lambda_{T_{-} T 3}=P 1 * P 2-\lambda_{T_{-} H}
\end{aligned}
$$

The probability of recovering from only one toxicity is equal to the total probability of recovering of that toxicity subtracted by the probability of recovering from that toxicity in combination with any other toxicity.

$$
\begin{aligned}
& \lambda_{T \_T 23}=P 1-\lambda_{T_{-T 2}}-\lambda_{T_{-T 3}}-\lambda_{T_{-} H} \\
& \lambda_{T_{-T 13}}=P 2-\lambda_{T_{-T 1}}-\lambda_{T_{-T 3}}-\lambda_{T_{-} H} \\
& \lambda_{T_{-T 12}}=P 3-\lambda_{T_{-T 1}}-\lambda_{T_{-T 2}}-\lambda_{T_{-} H}
\end{aligned}
$$

Next we want to calculate the state transition probabilities from a combination of two toxicities to healthy or to a single toxicity, as shown in Supplementary Figure S7.3 below. 


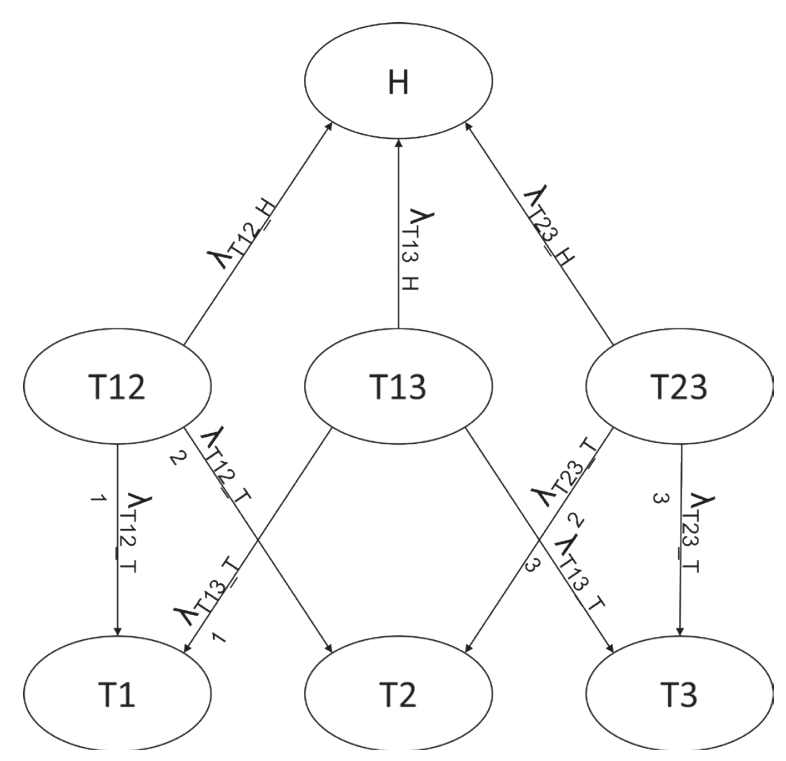

Supplementary Figure S7.3: A diagram showing the state transitions from any combination between two toxicities to the no toxicity health state and a single toxicity heath state

The transition probability of any combination of two toxicities two healthy is multiplication of the total probability of recovering of either toxicity:

$$
\begin{aligned}
& \lambda_{T 12 \_H}=P 1 * P 2 \\
& \lambda_{T 13 \_H}=P 1 * P 3 \\
& \lambda_{T 23_{\_} H}=P 2 * P 3
\end{aligned}
$$

The probability of recovering from one of the two toxicities is the total probability of recovering from that toxicity subtracted by the probability of recovering from both toxicities.

$$
\begin{aligned}
& \lambda_{T 12 \_T 1}=P 1-\lambda_{T 12 \_H} \\
& \lambda_{T 12 \_T 2}=P 1-\lambda_{T 12 \_H} \\
& \lambda_{T 13 \_T 1}=P 1-\lambda_{T 13 \_H} \\
& \lambda_{T 13 \_T 3}=P 3-\lambda_{T 13 \_H} \\
& \lambda_{T 23 \_T 2}=P 2-\lambda_{T 23 \_H} \\
& \lambda_{T 23 \_T 3}=P 3-\lambda_{T 23 \_H}
\end{aligned}
$$

Finally, the most straightforward transition probability is the probability of recovering from a single toxicity, which is equal to the total probability of recovering from that toxicity, as shown in Supplementary Figure S7.4. 


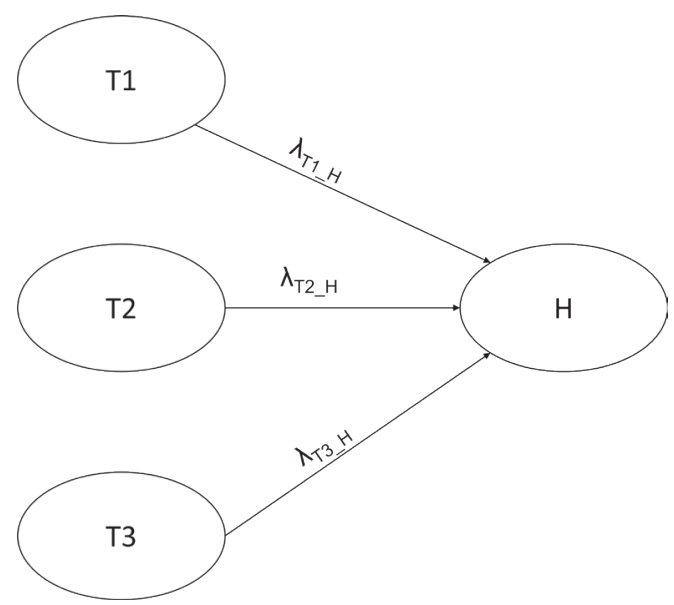

Supplementary Figure 57.4: The diagram showing the transition from a single toxicity health states to the no toxicity heath state.

Therefore:

$$
\begin{aligned}
& \lambda_{T 1 \_H}=P 1 \\
& \lambda_{T 2 \_H}=P 2 \\
& \lambda_{T 3 \_H}=P 3
\end{aligned}
$$

For external beam radiotherapy, patients start without toxicity and develop late toxicity, so the equations are the opposite.

\section{Supplementary Information S7.2: Utility values and costs for Markov model}

An overview of the utility values, obtained from Steward et al. are shown in Supplementary Table S7.1.

Supplementary Table S7.1: Utility values used in the Markov model

\begin{tabular}{l|ll} 
State & Expected value & SD \\
\hline Post-treatment, no adverse effects & Baseline & - \\
$E D$ & 0.89 & 0.16 \\
$U I$ & 0.83 & 0.21 \\
$R B$ & 0.71 & 0.26 \\
$U I+B T$ & 0.70 & 0.24 \\
$E D+B T$ & 0.57 & 0.26 \\
ED+UI & 0.79 & 0.23 \\
UI+ED+BT & 0.45 & 0.31 \\
Recurrence & 0.81 & 0.18 \\
Metastatic disease & 0.25 & 0.11 \\
\hline
\end{tabular}

ED: erectile dysfunction; UI: Urinary incontinence; RB: rectal bleeding; SD: standard deviation 
An overview of the costs are shown in Supplementary Table S7.2.

Supplementary Table 57.2: Costs used in the Markov model

\begin{tabular}{l|ll}
\multicolumn{1}{l}{ Description } & Mean costs $(\boldsymbol{\epsilon})$ & SD $(\boldsymbol{\epsilon})$ \\
\hline Open prostatectomy & 9780 & 978 \\
Radiotherapy & 8275 & 827.5 \\
\hline & Mean costs per year $(\boldsymbol{\epsilon})$ & SD $(\boldsymbol{\epsilon})$ \\
\hline Follow-up (year 1-2) & 511 & 30 \\
Follow-up (year >2) & 255 & 21 \\
Add. costs erectile dysfunction & 60 & 9 \\
Add. costs urinary incontinence & 303 & 21 \\
Add. costs rectal bleeding & 95 & 10 \\
Recurrence & 2535 & 212 \\
Metastatic disease & 2288 & 252 \\
Castrate resistant metastasis & 6493 & 313 \\
\hline
\end{tabular}

$S D=$ standard deviation

These values were obtained using the care product codes of the relevant treatments. We used the costs published on https://www.opendisdata.nl, and we used a standard deviation of $10 \%$ of the total costs of the treatment (Supplementary Table S7.3).

Supplementary Table S7.3: Treatment costs

\begin{tabular}{l|ll} 
& \multicolumn{1}{c}{ Total costs } & Care product code \\
\hline Radiotherapy & $€ 8275$ & 990061072,990061014 \\
Open Prostatectomy & $€ 9780$ & 20109012
\end{tabular}

Costs of treatments less than $€ 850$ were extracted using the care product code and the tariff tool developed by CZ (https://www.cz.nl/service-en-contact/ zoek-tarieven). For post-treatment costs we assumed 4 consultations and prostate specific antigen (PSA) tests per anno in the first two years, and 2 per anno after that (Supplementary Table S7.4).

Supplementary Table S7.4: Post-treatment costs

\begin{tabular}{|c|c|c|c|c|c|c|}
\hline & \multicolumn{2}{|c|}{ Quantity p.a } & \multirow[t]{2}{*}{ Price/unit } & \multicolumn{2}{|l|}{ Total costs } & \multirow[t]{2}{*}{ Product code } \\
\hline Year & $1-2$ & $>2$ & & $1-2$ & $>2$ & \\
\hline Consultation & 4 & 2 & $€ 122(15)$ & $€ 488$ (30) & $€ 244$ (21) & 20109088 \\
\hline PSA test & 4 & 2 & $€ 5,8$ & $€ 23,2$ & $€ 11,6$ & 72621 \\
\hline Total & & & & $€ 511,2(30)$ & $€ 255,6(21)$ & \\
\hline
\end{tabular}


Costs of medication was taken from a website, https://www. farmacotherapeutischkompas.nl, that reports the costs of pharmaceutical healthcare. At the suspicion of biochemical failure or metastatic disease, a PSMA scan can be made. If recurrence has taken place, the patient can receive secondary localized treatment, or hormonal therapy. Once the patient progresses to castrate resistant metastatic disease, the patient is treated with chemotherapy (Supplementary Table S7.5). 
Supplementary Table 57.5: Costs metastatic disease

\begin{tabular}{l|llll}
\multicolumn{2}{c}{ Quantity p.a } & Price/unit (SD) & Total costs (SD) & Product code \\
\hline $\begin{array}{l}\text { Consultation } \\
\begin{array}{l}\text { LHRH agonist } \\
\text { leuprorelin }\end{array}\end{array}$ & 4 & $€ 122(15)$ & $€ 488(21)$ & 20109088 \\
$\begin{array}{l}\text { Docetaxel } \\
\text { Prednisolon }\end{array}$ & 6 & $€ 450(125)$ & $€ 1800(250)$ & 20109010 \\
PSMA-scan & 6 & $€ 721(120)$ & $€ 4326(294)$ & 20109050 \\
Zoledronic acid & 0,35 & $€ 18$ & $€ 108$ & \\
\hline Total & 4 & $€ 1060(123)$ & $€ 371(73)$ & 120501 \\
& & $€ 422(40)$ & $€ 1688(80)$ & \\
\hline
\end{tabular}

SD: standard deviation; p.a; per anno

For the management of late erectile dysfunction, we assumed that half the patients use medication, $10 \%$ of the patients use cavernous injections and $5 \%$ use a vacuum pump. We estimated that medication or injections would be used on average once a month, and that the vacuum pump lasts two years (Supplementary Table S7.6).

Supplementary Table S7.6: Costs of the management of erectile dysfunction

\begin{tabular}{l|lll} 
& \multicolumn{1}{c}{ Quantity $\boldsymbol{p} . \boldsymbol{a}$} & Price/unit & Total costs \\
\hline Sildenafil & 6 & $€ 6,83(2)$ & $€ 41(5)$ \\
Cavernous injection & 1,2 & $€ 11,06(3)$ & $€ 13(3)$ \\
Vacuum pump & 0,025 & $€ 235(35)$ & $€ 6(6,5)$ \\
\hline Total & & & $€ 60(9)$
\end{tabular}

For the management of late urinary incontinence, we estimate that approximately three quarters of patients use physiotherapy, with 8 sessions per year. We estimate that $90 \%$ of the patients with urinary incontinence use pads 3 times a day. We assume that $5 \%$ requires diapers once a day, and all patients who use diapers or pads require net trousers. It is estimated that $2,5 \%$ require drainage bags, of which they will require bed bags and leg bags (Supplementary Table S7.7). For the prices of medical products such as pads, net trousers and leg bags, we collected costs from different websites such as www.mediqcombicare.nl, www. pharmamarket.nl, and www.123drogisterij.nl. 
Supplementary Table S7.7: Costs of the management of urinary incontinence

\begin{tabular}{l|lll}
\multicolumn{1}{c}{ Quantity $\boldsymbol{p .} \boldsymbol{a}$} & Price/unit & Total costs \\
\hline Physiotherapy & 1 & $€ 35(0,6)$ & $€ 35(0,6)$ \\
Balloon catheter & 0,05 & $€ 12(0,3)$ & $€ 0,6(0,07)$ \\
Pads & 394 & $€ 0,36(1)$ & $€ 142(20)$ \\
Diapers & 18 & $€ 0,65(0,08)$ & $€ 11,7(0,3)$ \\
Net trousers & 0,95 & $€ 1,25(0,1)$ & $€ 1,2(0,1)$ \\
Bed bag, sterile & 3 & $€ 20(0,7)$ & $€ 60(1,2)$ \\
Leg bag, sterile & 1,3 & $€ 40(1)$ & $€ 52(1,1)$ \\
\hline Total & & & $€ \mathbf{3 0 2 , 5 ( 2 1 )}$
\end{tabular}

Rectal bleeding is mostly treated with low cost treatments such as medication, but a low percentage has severe rectal bleeding and needs to be treated with infrared coagulation or a coloscopy (Supplementary Table S7.8).

Supplementary Table S7.8: Costs of the management of rectal bleeding

\begin{tabular}{l|llll} 
& Quantity p.a & Price/unit & Total costs & Product code \\
\hline $\begin{array}{l}\text { Salofalk/Arestal } \\
\text { Sclerosis, infrared }\end{array}$ & 36,5 & $€ 1,13(1)$ & $€ 41(6)$ & \\
coagulation & 0,05 & $€ 167(3,8)$ & $€ 8(0,8)$ & 35135 \\
$\begin{array}{l}\text { Intervention-coloscopy } \\
\text { Sigmoidoscopy }\end{array}$ & 0,05 & $€ 469(7)$ & $€ 23,5(1,6)$ & 34697 \\
\hline Total & 0,05 & $€ 445(34)$ & $€ 22(8)$ & 34686 \\
\hline
\end{tabular}

Supplementary Table S7.9: Distribution for the generation of synthetic dataset

\begin{tabular}{l|llll}
\multicolumn{1}{l}{ Parameter name } & Distribution type & Ref \\
\hline Age (years) & Normal & $\mu=60$ & $\sigma=10$ & {$[1-5]$} \\
PSA $(\mathrm{ng} / \mathrm{mL})$ & Beta & $\alpha=4.17$ & $\beta=51.5$ & {$[1-5]$} \\
BMI $(\mathrm{kg} / \mathrm{m} 2)$ & Normal & $\mu=28$ & $\sigma=3$ & {$[3-5]$} \\
T-stage & Step & $\mathrm{T} 1=66 \%$ & $\mathrm{~T} 2=34 \%$ & {$[1,3-5]$} \\
neg. biopsy cores $(\mathrm{N})$ & Normal & $\mu=4.5$ & $\sigma=1.9$ & {$[2]$} \\
pos. biopsy cores $(\mathrm{N})$ & Half normal & Location $=1$ & Scale $=2.46$ & {$[2]$} \\
P. Gleason grade & Beta & $4=14 \%$ & $3=86 \%$ & {$[1,3,4]$} \\
S. Gleason grade & Step & $4=19 \%$ & $3=71 \%$ & {$[2]$} \\
ADT given & Step & no $=22 \%$ & yes $=78 \%$ & {$[1,7]$} \\
ADT length & Beta & $\alpha=0.125$ & $\beta=1.25$ & {$[1]$} \\
ASA score & Step & $\mathrm{I} / \mathrm{II}=18 \%$ & $\mathrm{III} / \mathrm{IV}=82 \%$ & {$[1,3]$}
\end{tabular}


Nerve sparing

Abdominal surgery

HRQOL (\%)

Membranous urethra length $(\mathrm{mm})$

Mean Trigone dose (Gy)

V75 rectum (\%)

\begin{tabular}{|llll} 
Step & no $=81 \%$ & yes $=19 \%$ & {$[4]$} \\
Step & no $=90.3 \%$ & yes $=9.7 \%$ & {$[6]$} \\
Beta & $\alpha=1.5$ & $\beta=6$ & {$[5]$} \\
Normal & $\mu=12$ & $\sigma=3$ & {$[4]$} \\
Normal & $\mu=68$ & $\sigma=6$ & {$[3]$} \\
Gamma & location $=1$ & scale $=4$ & {$[6]$}
\end{tabular}

Ref: reference; PSA: prostate specific antigen; BMI: body mass index; T-stage: Tumor stage; N neg.: number of negative; N pos.: number of positive; P.: Primary; S.: Secondary; ADT: androgen deprivation therapy; ASA: American Society of Anesthesiologists; PN: Pelvic nodes; HRQOL: sexual health-related quality of life; V75: percentage receiving at least 75 Gray; Gy = Gray

1. Warner A, Pickles T, Crook J, et al (2015) Development of ProCaRS Clinical Nomograms for Biochemical Failure-free Survival Following Either Low-Dose Rate Brachytherapy or Conventionally Fractionated External Beam Radiation Therapy for Localized Prostate Cancer. Cureus 7:e276

2. Bjartell A, Bottai M, Persson J, et al (2016) Prediction of clinical progression after radical prostatectomy in a nationwide population-based cohort. Scandinavian Journal of Urology 50:255-259

3. Schaake W, van der Schaaf A, van Dijk LV, et al (2018) Development of a prediction model for late urinary incontinence, hematuria, pain and voiding frequency among irradiated prostate cancer patients. PLoS One 13:e0197757

4. Matsushita K, Kent MT, Vickers AJ, et al (2015) Preoperative predictive model of recovery of urinary continence after radical prostatectomy. BJU Int 116:577-583

5. Alemozaffar M, Regan MM, Cooperberg MR, et al (2011) Prediction of erectile function following treatment for prostate cancer. JAMA 306:1205-1214

6. Valdagni R, Kattan MW, Rancati T, et al (2012) Is it time to tailor the prediction of radio-induced toxicity in prostate cancer patients? Building the first set of nomograms for late rectal syndrome. Int J Radiat Oncol Biol Phys 82:1957-1966 
Supplementary Table S7.10: distribution of clinical parameters in synthetic dataset

\begin{tabular}{|c|c|c|c|}
\hline Parameter name & $E B R T(S D)$ & $R P(S D)$ & $P$ \\
\hline Age (years) & $63.8(10.7)$ & $58.8(9.1)$ & $<<0.001$ \\
\hline$P S A(n g / m L)$ & $8.3(3.5)$ & $6.8(3.5)$ & $<<0.001$ \\
\hline$B M I(\mathrm{~kg} / \mathrm{m} 2)$ & $27.9(2.9)$ & $28.0(3.0)$ & 0.81 \\
\hline T-stage $2(\%)$ & 48.7 & 24.4 & $<<0.001$ \\
\hline neg. biopsy cores (N) & $3.7(1.8)$ & $4.8(1.8)$ & $<<0.001$ \\
\hline pos. biopsy cores (N) & $3.5(1.7)$ & $2.7(1.4)$ & $<<0.001$ \\
\hline P. Gleason grade 4 (\%) & 27.1 & 3.3 & $<<0.001$ \\
\hline S. Gleason grade 4 (\%) & 14.7 & 20.9 & 0.01 \\
\hline ADT given (\%) & 79.8 & 0 & $<<0.001$ \\
\hline ADT length (months) & $19.0(27.5)$ & 0 & $<<0.001$ \\
\hline ASA score $>\| /(\%)$ & 86.2 & 78.9 & 0.003 \\
\hline Nerve sparing (\%) & 12.4 & 27.5 & 0.001 \\
\hline Abdominal surgery (\%) & 6.4 & 11.6 & 0.005 \\
\hline HRQOL (\%) & $82.9(12.3)$ & $76.9(14.6)$ & $<<0.001$ \\
\hline Membranous urethra length $(\mathrm{mm})$ & $11.8(3.0)$ & $12.1(2.8)$ & 0.04 \\
\hline Mean Trigone dose (Gy) & $67.2(6.0)$ & $68.4(5.7)$ & 0.001 \\
\hline V75 rectum (\%) & $3.8(3.9)$ & $4.5(4.6)$ & 0.008 \\
\hline
\end{tabular}

EBRT: external beam radiotherapy; RP: radical prostatectomy; SD: standard deviation; PSA: prostate specific antigen; BMI: body mass index; T-stage: Tumor stage; $N$ neg.: number of negative; N pos.: number of positive; P.: Primary; S.: Secondary; ADT: androgen deprivation therapy; ASA: American Society of Anesthesiologists; PN: Pelvic nodes; HRQOL: sexual healthrelated quality of life; V75: percentage receiving at least 75 Gray; Gy = Gray 
Supplementary Table S7.11: Benchmark study including clinical parameters reported

\begin{tabular}{|c|c|c|c|c|c|c|}
\hline Study & Treatment [N] & Parameters & & Outcome & Value & Model \\
\hline \multirow[t]{2}{*}{$\begin{array}{l}\text { Hamdy } \\
2016\end{array}$} & EBRT [545] & $\begin{array}{l}\text { Age (years) } \\
\text { GS } 7 \\
\text { T2PSA <10 } \\
\text { PSA ng/ml }\end{array}$ & \begin{tabular}{l|}
62 \\
$21 \%$ \\
$25 \%$ \\
$90 \%$ \\
4.7
\end{tabular} & 5 year BDFS & $88.5 \%$ & $89.0 \%$ \\
\hline & RP [553] & $\begin{array}{l}\text { Age (years) } \\
\text { GS } 7 \\
\text { T2 } \\
\text { PSA <10 P } \\
\text { SA ng/ml }\end{array}$ & $\begin{array}{l}62 \\
21 \% \\
25 \% \\
90 \% \\
4.7\end{array}$ & 5 year BDFS & $88.1 \%$ & $87.0 \%$ \\
\hline \multirow[t]{3}{*}{ CHHiP } & 74 Gy [1065] & $\begin{array}{l}\text { Age (years) } \\
\text { T2 } \\
\text { GS } 7 \\
\text { ADT } \\
\text { ADT months }\end{array}$ & $\begin{array}{l}69 \\
58 \% \\
64 \% \\
83 \% \\
6.2\end{array}$ & 5 year BDFS & $88.5 \%$ & $88.4 \%$ \\
\hline & 60 Gy [1074] & $\begin{array}{l}\text { Age (years) } \\
\text { T2 } \\
\text { GS } 7 \\
\text { ADT } \\
\text { ADT months }\end{array}$ & $\begin{array}{l}69 \\
58 \% \\
63 \% \\
85 \% \\
6.2\end{array}$ & 5 year BDFS & $91.3 \%$ & $89.9 \%$ \\
\hline & 57 Gy [1077] & $\begin{array}{l}\text { Age (years) } \\
\text { T2 } \\
\text { GS } 7 \\
\text { ADT } \\
\text { ADT months }\end{array}$ & $\begin{array}{l}69 \\
58 \% \\
65 \% \\
84 \% \\
6.2\end{array}$ & 5 year BDFS & $86.8 \%$ & $87.9 \%$ \\
\hline \multirow[t]{2}{*}{$\begin{array}{l}\text { Donovan } \\
2016\end{array}$} & EBRT [545] & $\begin{array}{l}\text { Age (years) } \\
\text { GS } 7 \\
\text { T2 } \\
\text { PSA (ng/ml) }\end{array}$ & $\begin{array}{l}62 \\
20 \% \\
21 \% \\
4.8\end{array}$ & $\begin{array}{l}6 \text { month UI } \\
6 \text { year UI } \\
6 \text { month ED } \\
6 \text { year ED } \\
6 \text { month RB } \\
6 \text { year RB }\end{array}$ & $\begin{array}{l}5 \% \\
3.5 \% \\
77.8 \% \\
72.6 \% \\
3.8 \% \\
5.6 \%\end{array}$ & $\begin{array}{l}3.3 \% \\
3.3 \% \\
85.4 \% \\
71.6 \% \\
14.7 \% \\
5.7 \%\end{array}$ \\
\hline & RP [553] & $\begin{array}{l}\text { Age (years) } \\
\text { GS } 7 \\
\text { T2 } \\
\text { PSA (ng/ml) }\end{array}$ & $\begin{array}{l}62 \\
22 \% \\
26 \% \\
4.9\end{array}$ & $\begin{array}{l}6 \text { month UI } \\
6 \text { year UI } \\
6 \text { month ED } \\
6 \text { year ED }\end{array}$ & $\begin{array}{l}46 \% \\
17 \% \\
88 \% \\
83.5 \%\end{array}$ & $\begin{array}{l}41.5 \% \\
17.0 \% \\
92.7 \% \\
83.7 \%\end{array}$ \\
\hline
\end{tabular}

$N$ : number of patients; EBRT: external beam radiotherapy; RP: radical prostatectomy; BDFS: biological disease free survival; UI: urinary incontinence; ED: erectile dysfunction; RB: rectal bleeding; Gy: gray; GS: gleason score; PSA: prostate specific antigen in ng/ml; ADT: androgen deprivation therapy

Supplementary Information 57.3: Sensitivity and scenario analysis Costeffectiveness analysis

Univariate one-way sensitivity analyses were performed to examine the impact of individual parameters on the results, of which the fifteen most impactful 
parameters are graphically represented in a tornado plot (Supplementary Figure S7.5). In the diagram we varied each parameter between the 2.5 and 97.5 percentiles and calculated the resulting iNMB of the DSS compared to the randomized treatment allotment strategy. The most impactful parameter is the utility value of erectile dysfunction, but over the range of these parameters the iNMB of the DSS was still positive, and thus cost-effective when compared to the randomized strategy.

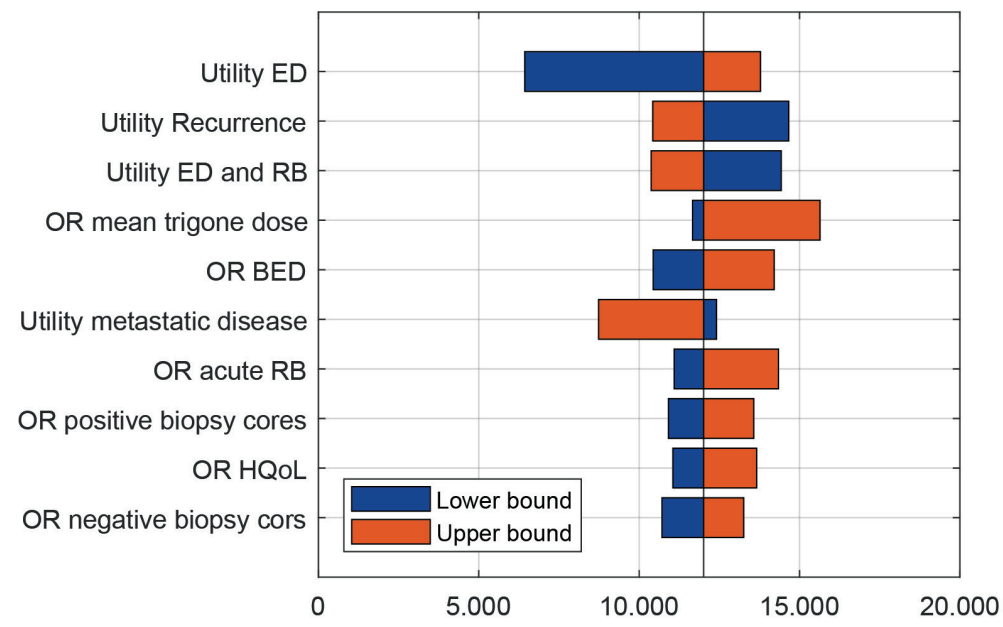

Supplementary Figure 57.5: This figure shows the impact of individual parameters on the incremental net monetary benefit of a decision support system versus randomized treatment. The vertical line represents the deterministic run. $E D=$ erectile dysfunction; $O R$ = odds ratio; $B E D=$ biologically effective dose; $R B=$ rectal bleeding; $H R Q O L=$ health related quality of life

To test the robustness of the model results, we varied some key assumptions in the model, of which the results are shown in Supplementary Table S7.12. Over the different scenarios the DSS kept a high probability of being cost-effective. 
Supplementary Table S7.12: The results of the different scenario analyses

\begin{tabular}{|c|c|c|c|c|c|}
\hline Scenario & Value & $\begin{array}{l}\text { TCP diff (\%) } \\
{[95 \% \mathrm{Cl}]}\end{array}$ & $\begin{array}{l}\text { Cost diff }(€) \\
{[95 \% \mathrm{Cl}]}\end{array}$ & $\begin{array}{l}\text { QALY diff (years) } \\
{[95 \% \mathrm{Cl}]}\end{array}$ & P CE (\%) \\
\hline \multirow[t]{3}{*}{ Discount rate } & $0 \%$ & $1.5[-2.9-5.9]$ & $-478[-657--324]$ & $0.15[-0.01-0.29]$ & 99.2 \\
\hline & $5 \%$ & $1.5[-2.9-5.9]$ & $-296[-396--196]$ & $0.09[0.01-0.18]$ & 99.8 \\
\hline & $10 \%$ & $1.5[-2.9-5.9]$ & $-200[-265--135]$ & $0.07[0.00-0.13]$ & 98.6 \\
\hline \multirow[t]{3}{*}{ Dose plan1 } & $2.5 \times 28$ & $1.8[-3.6-7.3]$ & $-614[-1376--148]$ & $0.09[-0.22-0.41]$ & 79.4 \\
\hline & $2 \times 35$ & $2.0[-2.2-6.2]$ & $-147[-832-538]$ & $0.19[-0.19-0.56]$ & 88.0 \\
\hline & $2 \times 37$ & $1.5[-2.9-5.9]$ & $-308[-425--192]$ & $0.13[0.01-0.25]$ & 99.4 \\
\hline \multirow[t]{4}{*}{ Time horizon } & 5 & 1.5 [-2.9-5.9] & $-78[-106-50]$ & $0.03[-0.01-0.07]$ & 97.2 \\
\hline & 10 & $1.5[-2.9-5.9]$ & $-173[-229--117]$ & $0.07[-0.00-0.15]$ & 99.2 \\
\hline & 15 & $1.5[-2.9-5.9]$ & $-260[-347--172]$ & $0.09[0.00-0.18]$ & 99.0 \\
\hline & 50 & $1.5[-2.9-5.9]$ & $-418[-561--274]$ & $0.17[-0.00-0.35]$ & 98.6 \\
\hline \multirow[t]{4}{*}{ Costs DSS } & 20 & $1.5[-2.9-5.9]$ & $-304[-414--194]$ & $0.12[0.01-0.23]$ & 99.6 \\
\hline & 100 & $1.5[-2.9-5.9]$ & $-224[-334--114]$ & $0.17[-0.0-0.33]$ & 98.6 \\
\hline & 250 & $1.5[-2.9-5.9]$ & $-74[-184-26]$ & $0.17[-0.0-0.33]$ & 99.4 \\
\hline & 500 & $1.5[-2.9-5.9]$ & 176 [66-286] & $0.17[-0.0-0.33]$ & 99.4 \\
\hline
\end{tabular}

$C l=$ confidence interval; $P=$ probability; $E B R T=$ external beam radiotherapy; $D S S=$ decision support system

1 Fraction dose $x$ the number of fractions
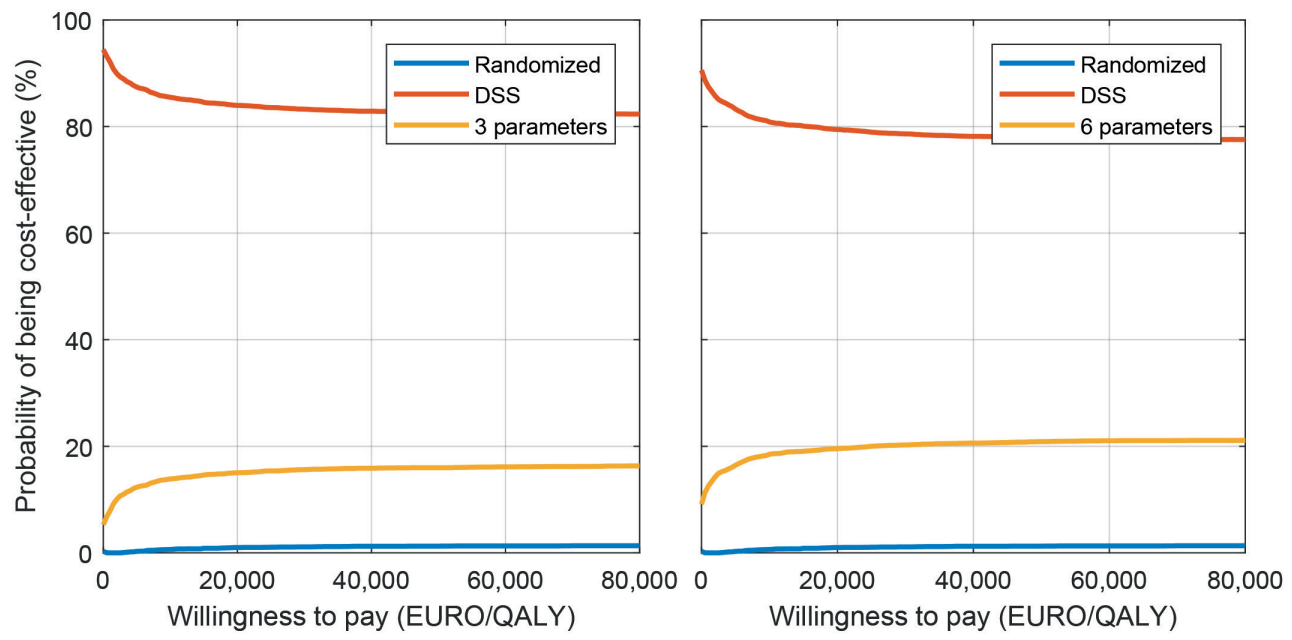

Supplementary Figure S7.6: the cost-effectiveness acceptability curve (CEAC) comparing the randomized treatment allotment strategy to the full decision support system (DSS), and a simplified DSS that utilized only age, prostate specific antigen (PSA) and T-stage. 
To test the usefulness of our advanced DSS, we tested a simplified DSS using only three clinical parameters: age, t-stage, and PSA. The simplified DSS had a large probability (85.1\%) of being cost-effective compared to the randomized treatment allotment strategy. However, compared to the original DSS, the simplified DSS only had a $16.3 \%$ chance of being cost-effective. We expanded the simplified DSS with three extra parameters, including primary Gleason score, diabetes and prior abdominal surgery, and found that this simplified DSS had a $89.1 \%$ probability of being cost-effective compared to the randomized strategy, so $4 \%$ better than the 3 parameter DSS. However, this 6 parameter DSS still only outperforms the full DSS in $21.1 \%$ of the simulations (Supplementary Figure S7.6). 
Discussion 

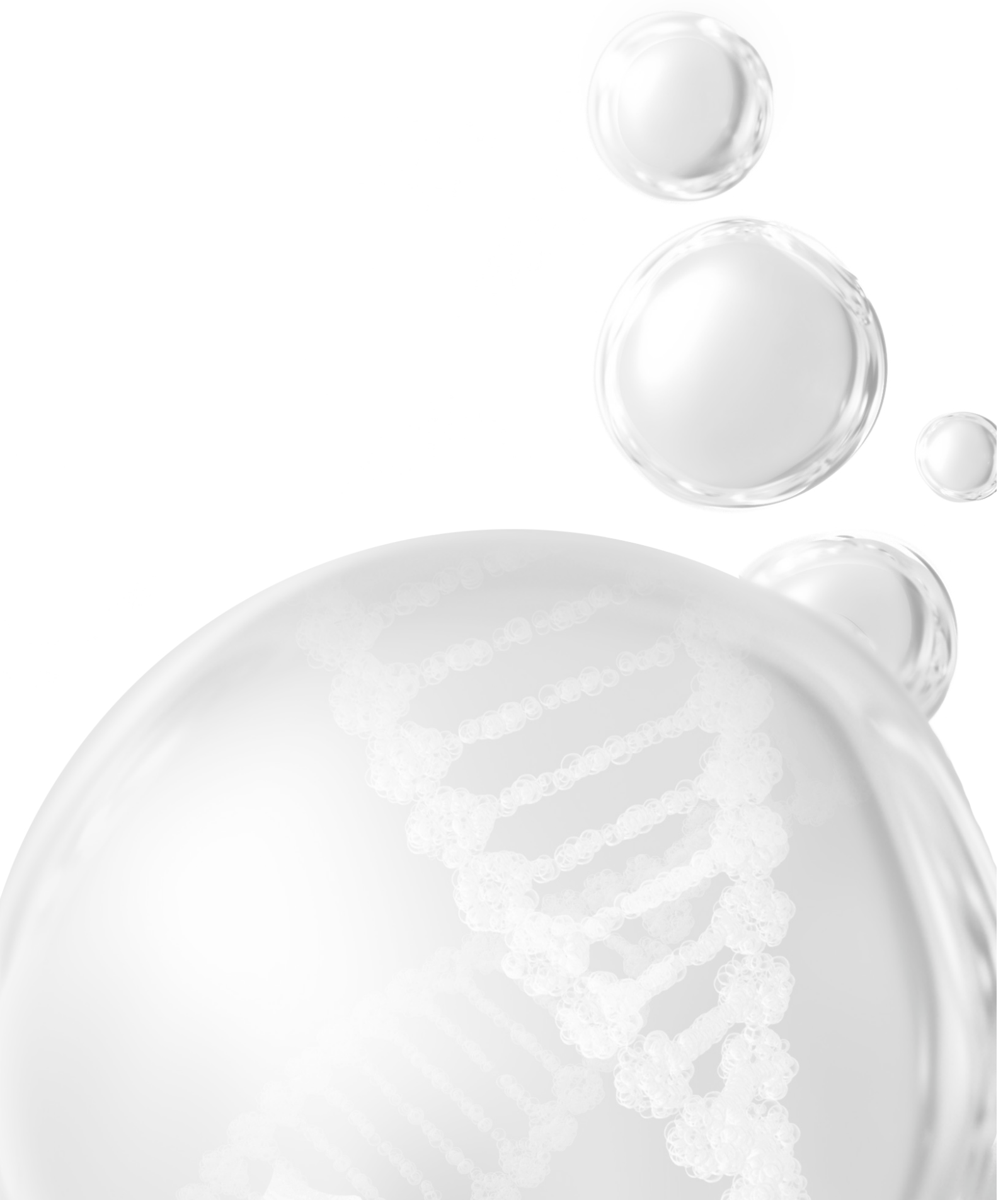


\section{Chapter 8}

General Discussion and future perspectives 


\section{General discussion}

The focus of this thesis was on the development of clinical decision support systems (DSS) for the treatment selection for low-intermediate prostate cancer patients, a group of patients for whom a variety of treatments provide comparable outcomes in terms of (progression-free) survival but which vary in possible treatment related side-effects. The development of DSS is an important part of rapid learning healthcare, a process described in Chapter $\mathbf{1}$, and is a crucial step to bringing machine learning based models that predict outcome into clinical practice.

The overall aim of this thesis, as defined in Chapter $\mathbf{2}$, is as follows:

"Bridging the gap between machine learning based models and the decision making process through the development of clinical decision support systems for prostate cancer patients"

This aim was subdivided into two objectives:

Objective 1: To provide an overview of decision support systems in prostate cancer treatment

Objective 2: $\quad$ To develop and challenge new clinical decision support systems that aid in the treatment selection of prostate cancer both a) within the field of radiotherapy and b) multidisciplinary.

We defined a number of primary hypotheses for this work:

1. Image deformation can be used to create a CT scan of a patient with an implantable rectum spacer prior to implantation, allowing for dose comparisons.

2. A clinical decision support system can be used to identify patients for whom an implantable rectum spacer is beneficial prior to treatment.

3. The expected biochemical failure-free survival of patients can be improved when using an iso-toxic DSS to decide on the implantation of an implantable rectum spacer during external beam radiotherapy.

4. The expected quality of life and cost-effectiveness of patients can be improved when using a clinical decision support system to decide between external beam radiotherapy and prostatectomy for the treatment of prostate cancer.

In the sections below we will present a motivated view of the methods used in this thesis and how they worked towards meeting our objectives as well as our primary findings and how they reflect on our hypotheses. This will be followed by an analysis of the primary implications of our findings and suggestions for future works. 


\section{Main findings}

Prior developments

In Chapter 3, we provide an overview of available literature concerning DSS for prostate cancer, which works towards addressing Objective 1. The DSS were split into three different types: diagnostic DSS, which were meant to support diagnosis of prostate cancer; treatment DSS, which were meant to aid in treatment selection; and patient decision aids (PDA), which were meant to aid decision making by the patient. In this chapter we found that DSS that support diagnosis and staging of prostate cancer were the most available type and had often been validated on large cohorts. Diagnostic DSS were found for assessing a variety of factors, including lymph node involvement, seminal vesical involvement, risk group, cancer stage, or extracapsular extension. DSS for treatment selection were less varied all focused around different types of external beam radiotherapy (EBRT), including proton versus photon therapy, stereotactic body radiotherapy versus intensity modulated radiotherapy and the use of rectum spacing devices during EBRT. As for PDA, this was shown to still be a very immature field of study with many challenges to be overcome, such as cultural and language barriers. However, the studies found do show that patients actively involved in the decision-making process experienced increased satisfaction and reduced anxiety and depression.

\section{Implantable rectum spacer}

Over the years, the benefit of dose-escalation during external beam radiotherapy (EBRT) for prostate cancer has become more and more evident, resulting in higher cure-rates ${ }^{[1,2]}$, however, the dose to the anorectum is one of the primary limiting factors in this development, as this can result in long term damage leading to bleeding and increased frequency ${ }^{[1]}$. Rectum separation devices, such as the implantable rectum spacer (IRS), protect the rectum from high dose levels by creating a distance between the anterior rectal wall and radiated prostate ${ }^{[2]}$. However, since the placement of an IRS does not come without additional costs ${ }^{[3]}$, possible risks and discomfort to the patient, and it has been proven not all patients stand to benefit from such a procedure ${ }^{[4]}$, a DSS to select patients for whom this would have clinical benefit is an important development. Building, expanding and challenging such a DSS was the focus of Chapter 4, 5 and $\mathbf{6}$, and addressed Objective $2 a$.

\section{Virtual spacer}

One of the challenges faced when developing a DSS for an IRS is that, in contrary to other EBRT DSS, it is not as straightforward to compare treatment plans, since a CT scan of a patient with IRS will not be available prior to treatment. Hypothesis 1 of this thesis was that image deformation can be used to create a CT scan of a 
patient with an IRS prior to implantation, allowing for dose comparisons. This was done in Chapter $\mathbf{4}$ by developing a virtual IRS (V-IRS) in the form of a vector field obtained from a set of CT scans of patients with rectal balloon implants. This vector field could then be applied to a CT scan, thus obtaining a synthetic CT scan of a patient with an IRS, a V-IRS.

Hypothesis 2 was that a DSS can be used to identify patients for whom an IRS is beneficial prior to treatment. We used the V-IRS as the basis for such a DSS comparing dose, probability of developing late rectal bleeding and costeffectiveness based on two RT treatment plans: one with and one without a spacer. We assessed the performance of the V-IRS by comparing the DSS outcomes to the outcomes when using a real CT scan of an IRS. The results showed that the V-IRS could not exactly replicate the real IRS results, but performed well in treatment classification: e.g. the real IRS and the V-IRS agreed in optimal treatment selection.

The study presented in this chapter has several limitations. The first one is that the model was only tested in a proof of concept study including 16 patients, and though the results were promising, the V-IRS and the DSS should be validated on a larger cohort. The advantage of this initial test was, however, that it included both hydrogel based IRS' and balloon implants, proving that the model had potential on both closed system IRS (balloon implant) and on liquid IRS (hydrogel). Another limitation is that the model for late rectal bleeding is dated, trained on older EBRT dose and delivery technologies. The used regression model also becomes less accurate when the probability of late rectal bleeding becomes lower than 4\%, which becomes problematic when applying it to the IRS, as in some cased the dose to the rectum is sufficiently reduced so that the risk of late rectal bleeding would be negligible. A significant barrier for the implementation of this DSS in clinical practice is the treatment planning step; in the study published in this chapter manual treatment planning was done for the CT without IRS, with V-IRS and with IRS, and in clinical practice this would result in a minimum of 2 treatment plans, and a maximum of 3 . This does not facilitate a streamlined clinical workflow.

\section{Expanding the DSS}

With the ever growing number of biomarkers predictive for treatment outcome being published, the chasm between scientific literature and clinical practice seems to be growing. Though the biomarkers could provide insight into patient specific treatment outcome, the number and the variety of them issues a new challenge. In Chapter 5, we proposed a method of bridging this chasm by incorporating published biomarkers, namely single nucleotide polymorphisms (SNPs) predictive for rectal toxicity into a Lyman Kutcher Burke (LKB) model ${ }^{[5]}$, without having to retrain the model. This was done using a mathematical approach that utilized the occurrence frequency of the biomarker and the odds ratio ${ }^{[6]}$. The advantage of this 
method is that biomarkers could be combined into existing models without having to retrain these models from scratch. This circumvents the limitation of needing clinical trials for whom these biomarkers as well as all the other parameters of the existing model have been documented, as this might not be feasible. This could aid in preventing valuable published biomarkers from being underutilized in clinical practice ${ }^{[7,8]}$.

The assessment of this method was only limited in this chapter, however, as only SNPS were integrated into an LKB model, and the challenge of incorporating biomarkers into other commonly used types of models, such as Cox regression models or Random Forrest models were not tested. Also the success of this method was not tested through validation on clinical data, and only the potential benefit was discussed and possible applications were indicated.

The DSS described in Chapter $\mathbf{4}$ was an iso-dose model, where both treatment plans were made with the same target dose to the target volume, and the effect on rectal dose and toxicity was further compared. In Chapter $\mathbf{5}$ we adapted the IRS DSS to be iso-toxic, where we optimized the target dose to result in an acceptable risk of rectal toxicity. We did this by performing treatment planning, and then scaling the dose volume histograms (DVHs) of the prostate, the anorectum and the bladder to mimic increasing or decreasing target dose until the expected risk of late rectal bleeding was within the target limit, or until bladder dose restraints were exceeded. The DSS was also expanded to include tumor control probability (TCP), but the cost-effectiveness comparison was excluded from the scope of this chapter.

One of the primary limitations of this method were that the full treatment plan was not remade after initial treatment planning, and only the DVH was scaled. This assumes that when increasing the target dose, the shape of the DVH to organs at risk does not significantly change, and only the magnitude varies. The cost-effectiveness comparison being dropped somewhat undermines the value of the original DSS as well.

\section{Challenging the V-IRS}

In Chapter 6 we challenged the robustness of the V-IRS by assessing the impact of rectal spacer dynamics on rectum dose and complication risk. It was shown that the exact shape of the IRS is not crucial in terms of expected toxicity, implicating that as long as there is sufficient distance between the anorectum and the prostate, the $\mathrm{V}$-IRS and real IRS would find similar reductions in risk of toxicity.

The primary limitations of this study were that the aim was not to assess the robustness of the V-IRS, but rather to assess the impact of natural balloon shrinkage over the treatment period. This had as a consequence that the changes in the rectal balloon volumes were limited to realistic balloon shrinkages, and no 
structural assessment of the impact of shape, size and placement location of the IRS was done.

\section{Prostatectomy versus radiotherapy}

For the treatment of low to intermediate risk prostate cancer patients, the two primary choices for active treatment are EBRT and radical prostatectomy (RP). Studies have shown that these treatments have similar outcomes in terms of curerate, but differ in types of toxicity and risk of toxicity. In Chapter $\mathbf{7}$ we developed an extensive DSS to predict the risk of recurrence, different combinations of treatment related toxicity, quality adjusted life-years (QALY's), and costs for both types of treatment (Figure 8.1). Since EBRT and RP are different disciplinary fields, this DSS is in line with Objective $2 b$. 

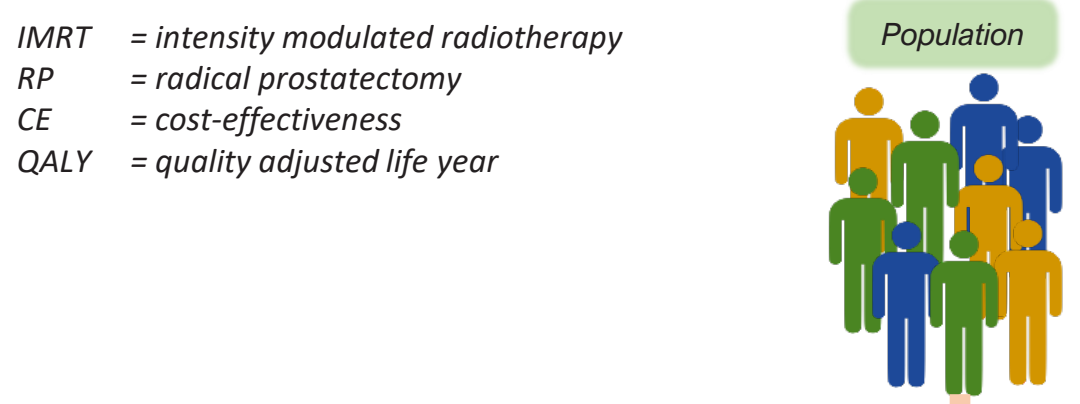

IMRT parameters

Progression risk

- Erectile dysfunction

- Urinary

incontinence

IMRT Outcomes

- Bowel problems

Treatment costs

-Follow-up and

toxicity costs

QALY's

IMRT CE

RP CE
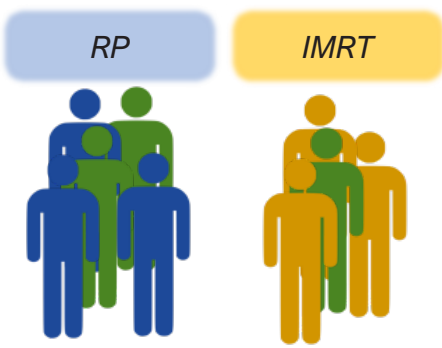

- Toxicity/Outcome

- Cost-effectiveness

- Patient preference

Comparison

Treatment selection

Figure 8.1 - Overview of the DSS for the selection of RP or EBRT for prostate cancer patients. 


\section{The DSS}

The DSS described in Chapter $\mathbf{7}$ had a number of strengths, among which were that it translates the risks of different toxicity types as well as the risk of disease progression into a single number, QALYs. This allows for an easy comparison, and has the potential to improve decision making, as the numerous clinical parameters, disease properties and treatment characteristics are condensed into a much more limited number of factors. The extensive nature of the model also makes it very suitable for expansion, for example to include the IRS into the EBRT arm. Despite the DSS being fully model-based, validation on published clinical trials showed that the outcomes reported there could be replicated with a good degree of accuracy, despite all the assumptions having to be made. This implicates that it could provide relevant information when generally applied. Also, similar to the framework described in the DSS for an IRS, this DSS allows the models incorporated in its structure to be exchanged relatively easily, meaning that if different models are available locally, or newer models become available, these can be used to specify or update the DSS. Hypothesis 4 was that the expected quality of life and costeffectiveness of patients improved with the use of a DSS to select EBRT or RP as primary treatment. We tested this hypothesis by performing a cost-utility analysis that compared a randomized treatment allotment strategy, or even a strategy using age, prostate specific antigen (PSA) and Gleason score, to the DSS. This showed that the DSS has the potential to not only improve health related outcome, but also reduce treatment costs.

The primary shortcoming of this DSS was how clinical practice was simulated after disease progression, namely that secondary treatment was always hormone therapy or chemotherapy ${ }^{[9]}$. Moreover, for clinical practice, this version of the DSS is unpractical, as in total 23 clinical, disease and treatment characteristics need to be available, among which MRI results and dose parameters. Future research should focus on addressing these limitations.

\section{"Synthetic patient data"}

In order to do the validation of the DSS as well as perform the cost-utility analysis, we required a patient dataset for whom all clinical parameters were available. Since no such dataset was available to us, we generated a synthetic patient dataset where patients consisted of a set of clinical, treatment and disease characteristics representative for the population. These characteristics were generated by identifying distributions found in published clinical trials, which were then used to randomly generate patients.

This method has some very significant benefits, first of which was that no actual patients were needed, and we were free to generate as many patients as needed. When validating against published clinical trials, the characteristics of the synthetic 
patient dataset were adjusted to match the parameters available for those trials, while parameters not made available were kept static. This made it possible to assess the accuracy of the DSS models without knowing all the available clinical parameters.

The biggest limitation of this method was that it was assumed there was no interaction between the characteristics, which we know to be false. For example, age is correlated to pre-treatment sexual function, while this is not simulate in the synthetic patient dataset. Another limitation of this approach is that published clinical trials were used as a basis, while those are not representative of the actual population. Though this is not a limitation that affects our validation approach, it does limit the value of the results from the cost-utility analysis.

Though this method should be improved though exploration of the interaction between clinical parameters, and the difference of parameters between the actual population and clinical trials, this method has a lot of potential, as demonstrated in the various applications for which it was used in this thesis. It is also an important step towards in silico clinical trials, which provide the opportunity to perform a "dry run" of a clinical trial prior to execution.

\section{In silico clinical trial}

Within the context of medicine, in silico refers to the direct use of computer simulations in simulating real biological processes. An in silico clinical trial consists of developing virtual cohorts to develop or evaluate a medicine or intervention. In Chapter 7 we combined the use of the previously described synthetic patient dataset with the models compiled in our DSS to develop an in silico clinical trial. The trial we designed was to compare the costs and effects of treating an elderly patient population (aged 75-80) with RP, EBRT or with a DSS based treatment allotment strategy. The results showed that for $72 \%$ of the elderly patients, the DSS advised EBRT as the optimal treatment strategy.

The limitations of the study described here is the limited application; we only applied this for a single patient population. However, our success in replicating published clinical trials suggests that within the restraints of the synthetic patient dataset, valuable information could be provided for sub-sets of patient groups.

\section{Implications for research and practice}

The work presented in this thesis has been done in order to improve the decision making process for prostate cancer patients using biomarkers and machine learning models. Specifically, the different objectives were set up to either aid in overcoming challenges or contributing to the volume of available technologies.

Within this thesis we proposed two different DSS: one for the placement of an IRS during EBRT, and one to compare RP and EBRT. The strengths of these DSS was the multi-level analysis that was obtained from a large number of inputs, providing 
comparisons in relevant dose metrics for both the target volume and the organs at risk, comparisons in cure rate and toxicities and providing a cost-effectiveness analysis. For both of these DSS existing predictive models were used to assess outcome in terms of progression free survival after treatment and risk of treatment related toxicity. One of the challenges doctors who want to use machine learning trained models are currently faced with is that new machine learning models are constantly being published. The quantity and varying quality of these models makes it difficult for them to be applied effectively in clinical practice, leading to many valuable models being underutilized. Using a DSS as proposed in this work, the selection and curation of clinically relevant prediction models has already been done and the clinical implications of these models is put into perspective through treatment comparisons and translation to QALYs. This holds especially true when external validation has been performed of the combination of these models. The way this DSS is developed offers the opportunity to replace these with more clinically relevant models as they become available, or as local clinical practice requires, which prevents the DSS from having to become outdated. Protocols should be followed to ensure that the models are applied correctly and that a certain quality is upheld. The work presented here offers platform that could function a bridge between literature and clinical practice through a comfortable and flexible structure. It could potentially offer clinicians a preselection of refined and combined models and biomarkers with a tool that aids them in optimized application of those models and biomarkers. Further evolution of this tool in replacing outdated models or including new biomarkers should be done using approved tools and checklists such as the CHARMS (critical appraisal and data extraction for systematic reviews of prediction modelling studies) checklist ${ }^{[10]}$, or the PROBAST (prediction model risk of bias assessment tool ${ }^{[11]}$ for proper curation and selection. This fits into our primary aim for our thesis in bridging the gap between machine learning models and individualized decision making.

In order to fit a variety of different machine learning models into a single framework, we applied a number of creative methods. The first step was to find relevant models that predict similar outcomes for different treatment types: e.g. we needed models to predict the risk of biochemical failure for both RP and EBRT, and the same with different toxicities. Not all models were published in a way that made them easily applicable, for instance, many models are published only as nomograms, and did not have the complete regression coefficients available in the paper. Using reverse engineering we extracted these coefficients from the nomogram. These models often predict the risk of developing an event after certain amount of time, for example the risk of developing biochemical failure 5 years after treatment. For some outcomes, this time period was different between the two treatment arms. In order to be able to make a long term comparison, a number of assumptions were made, such as the time after which the risk of events was 0 , the risk of and event 
directly after treatment, and the rate at which the risk changes over time. These assumptions were gathered from literature and applied to each model, and in this work we showed that despite being rough assumptions and interpolations, the trends followed clinical trial results moderately well.

In order to test our DSS for RP and EBRT, we developed a method of generating synthetic data. This has some interesting implications for research, as one of the challenges within medical research is obtaining large quantities of patient data. This is hampered by a number of factors, such as reluctance to share data (for example due to publication pressure or competing research), ensuring patient privacy in keeping with the general data protection regulation (GDPR) and monetary motivations. Clinical trials often provide a limited perspective of the patient population, as a large number of patients falls outside of inclusion criteria. Through our method of generating synthetic patients, we provide an aid in overcoming this challenge ${ }^{[12,13]}$. By resampling patient characteristics, it could be possible to completely synthesize patient populations and provide an alternative when sharing real data is not feasible. For this to be done well, it is recommended that correlation between clinical parameters and to outcome is carefully analyzed and taken into account. Another challenge is obtaining enough data of underrepresented sub-populations. This method could be used for data augmentation to increase the volume of these sub-groups. Such a method could be useful for example in the use of in-vitro clinical trials, as proposed in this thesis, as well as for cost-utility modeling. This could be used to assess the potential benefit of a medical procedure prior to clinical trials or aid in optimizing clinical trial design. An example would be to predict possible outcomes for sub-populations, which could be useful information when determining the inclusion criteria, or to assess which clinical parameters are likely to be clinically relevant. It could also aid in optimizing the desired study size with respect to the goal of the clinical trial. It could also be used to add onto clinical trial results in the form of data augmentation to improve heterogeneity of the population.

\section{Future perspectives}

Some very important steps towards the facilitation of RLHC have been made in this thesis, and the groundwork has been laid for future developments as well.

In Chapter 3 we found that that especially regarding DSS for treatment selection a large literature gap exists. Also the development of PDAs appear to be in an early phase but show promise.

In Chapter 4 we developed a V-IRS as the basis for a DSS. Before this DSS would be applicable in clinical practice, additional work needs to be done. The V-IRS would have to be critically analyzed through validation on a larger scale. Also, the method of applying the V-IRS, which is currently an arbitrarily chosen vector field should be scrutinized through comparison to different methods, such as a different vector 
field or a finite element based model[14]. The models included in the DSS should be updated and expanded to include tumor control probability, cancer specific survival, and perhaps erectile dysfunction models, as studies have suggested that the IRS can impact this as well[15]. An automatic treatment planning step would aid in streamlining the clinical integration as well ${ }^{[16,17]}$. The automatically generated treatment plan would not have to be used for the final treatment strategy, but could function to make a fast, fair comparison between the diagnostic $\mathrm{CT}$ and the synthetic V-IRS CT.

In Chapter $\mathbf{5}$ we expanded this DSS with additional biomarkers and by making the treatment selection strategy iso-toxic rather than iso-dose, but this chapter did not include a cost-utility analysis. An important next step for the improvement of this DSS would be to include a cost-effectiveness analysis, which would have to include treatment costs, and utility values and costs for follow-up treatment after recurrence. This difference with the iso-dose model arises because in the iso-dose model it can be assumed that the probability of biochemical failure is the same in both treatment arms, and thus this health state was omitted. Also, if dose escalation would be done by increasing the number of fractions, differences in treatment costs may arise. An improvement for the model would also be to include an automated treatment planning algorithm, as discussed before. This is even more important here, as this would allow the final comparison to be based on properly generated treatment plans, rather than scaled DVHs. Also, the method of including biomarkers should be tested on multiple types of machine learning based models, such as cox regression and random forest, and different methods should be explored. Methodical validation of these methods is vitally important before implementation on clinically applied models.

Chapter 6 challenged the robustness of the V-IRS by assessing impact of IRS dynamics on rectal toxicity. Though initial results are promising, a more extensive sensitivity analysis should be performed to assess the robustness of the V-IRS in terms of placement and shape. Also the impact on tumor control probability and the bladder toxicity should be taken into this analysis.

In Chapter $\mathbf{7}$ we developed a DSS for the treatment of prostate cancer with EBRT or RP. Direct future works would be to merge this DSS with the IRS DSS and expand to include different treatment modalities. This chapter also described a synthetic dataset. This concept should be further explored and validated. The in silico clinical trial that was done based on the synthetic dataset showed potential, but the application in this chapter was limited. Future works should include developing more in silico clinical trials, and replicating existing clinical trials for validation.

A more detailed discussion regarding future works is given in the sections below. 


\section{Improvement and expansion current models}

This thesis developed models for the IRS during EBRT, and made a comparison between EBRT and RP, however, other viable treatment options should be imbedded into these DSS in the future.

Brachytherapy is another active treatment that is being applied for prostate cancer with high success rates, and more models are becoming available that predict both outcome and toxicity, making it an excellent candidate for incorporation into this DSS ${ }^{[18-20]}$. The advantage of brachytherapy compared to EBRT is that the risk of rectal toxicity is much lower due to no additional treatment planning margins being needed to account for uncertainty, while applying higher doses to the region of interest. Also, the treatment is delivered in a single day, with no recovery time, as opposed to multiple treatments over a longer period of time. The disadvantage is that the risk of urinary toxicity is higher, and the patient will require anesthesia. Despite brachytherapy being a radiation based treatment, rectum spacing devices have less impact combined with brachytherapy as primary treatment, reducing mostly grade 1 rectal toxicity. However, studies have shown that during reirradiation some patients would stand to benefit from additional rectum spacing ${ }^{[21,}$ 22]. When the DSS is expanded to include follow-up treatment, the IRS could be included as an additional treatment option within the brachytherapy arm.

Another treatment decision that needs to be made, in particular for lowrisk prostate cancer patients, is whether to use an active treatment or not. Active surveillance is gaining popularity as a viable treatment option, but is not without risks ${ }^{[23,24]}$. The distinct advantage of active surveillance is that there are not treatment-related side effects, but the disadvantage is that there is a risk of progression. Patient selection for this passive treatment option is therefore very important in order not to reduce patient outcome while reducing treatment related side-effects from active treatment. The patient specific risk of progression during surveillance would be a critical model in order to expand the DSS with this treatment option. Also, follow-up treatment would have to be incorporated in case of progression, taking into account different active treatment options and the different treatment related side-effects.

In addition to expansion, the DSS for the IRS and the DSS for EBRT versus RP should be combined into a single framework. This integration will be seamless once automatic treatment planning is incorporated into the former.

The methods with which the DSS were developed used machine learning models published in literature and combined these into a single framework. In order to create a proper comparison and obtain transition probabilities for a Markov model to calculate the QALYS, assumptions were made and interpolation and extrapolation was done over time. Also, a number of models were only published as nomograms with no coefficients reported, and required reverse- 
engineering. This approach worked well for the scope of this thesis, however it is a sub-optimal approach. For the development of DSS using literature reported models, ideally all details of the models should be reported in publications, or even better, access to the data where the models were trained on. Adherence to the "Transparent reporting of a multivariable prediction model for individual prognosis or diagnosis" (TRIPOD) statement would aid in former of these solutions. The latter is a more complex matter, but the idea of Open Data ${ }^{[25]}$ and adhering to the guidelines written to improve Findability, Accessibility, Interoperability, and Reuse (FAIR) of digital assets ${ }^{[26]}$ would make future curation and combination of these models more reliable.

\section{Integration current models to clinical practice}

One of the large challenges this DSS will have to overcome is integration into clinical practice. Currently, the DSS presented in this thesis are not in a form ready for use by medical professionals, as they lack (external) validation, a proper user interface and certification from a regulatory body.

Prior to integration into the clinic, proper validation needs to be done of the DSS after the developments in the previous section have been completed. Since the models incorporated in the DSS should already be validated, it is the tool as a whole that should be subjected to multi-centric and international validation, where both doctors and patients should have the possibility to provide feedback on the additional value the DSS provides in the decision making process.

Currently the DSS exist only in the form of equations implemented in raw code. An important step that needs to be done is to create an intuitive, easy to use user interface that connects the target user to the framework. This should be both for regular application, e.g. support of treatment decision making, as well as for the selection of local models to embed into the DSS.

For this to be effective, it is crucial that the DSS is further developed in cooperation with clinical experts who can provide feedback on the needs of clinical practice. This will provide valuable feedback and prevent unusable technologies from being developed. This should be followed by try-outs in the form of prototypes that are offered to partnered hospitals or clinics in order to provide validation results.

Finally, the DSS should be subjected to proper certification, such as CE marking or FDA approval, to ensure safe usage and confidence from medical professionals. Once the model is determined to be clinically relevant and safe for use, clinical integration needs to take place. This will require a number of steps ${ }^{[27]}$, such as training medical professionals to effectively apply the DSS, develop a positive attitude and confidence towards the DSS from the clinic, set-up try-outs if clinical integration followed by full integration. The software should be maintained and the models will have to be kept up-to-date over periods of time. 


\section{Patient decision aids}

The DSS presented in this thesis have the aim of aiding the clinical professional in the decision-making process for the treatment of prostate cancer (PCa) patients. Although there is evidence that these DSS have the potential to improve patient quality of life (QoL) and reduce costs, it remains true that for a large group of patients, multiple treatment options have similar outcome ${ }^{[28]}$. For this group of patients, personal preferences, patient background and life-style come to dominate which treatment is the best fit. In these cases, shared-decision making (SDM) is a beneficial extension to the clinical decision-making process ${ }^{[29,30]}$.

In order to implement SDM, a number of challenges need to be overcome, of which the first one is informing the patient. In order to fully involve the patient in the decision for his treatment, he needs to be aware of the different treatment options for which he is eligible, and what the risks and benefits of each of these are. Unfortunately regular consultations are not sufficient for the transference of this information, as has been shown in previous studies ${ }^{[31]}$. This can result in underinformed decisions which impact QoL, or a biased decision due to the limited knowledge of specialists (urologist, radiologist) within the multi-disciplinary field of $\mathrm{PCa}^{[32]}$. Patient decision aids (PDAs) could be a way of addressing this issue. Using PDAs, the patient can receive both high level and in depth information regarding different treatment options considering procedure, outcomes and sideeffects, including side by side comparisons. This information can be presented in an accessible way and is not limited to the short consultation times that clinical professionals can offer.

This potential in PDAs has been recognized, and tools have previously been developed to provide the patient with additional information regarding treatment decision-making ${ }^{[3-35]}$. However, these do not take into account patient specific characteristics and do not aid in personalized decision-making, rather focusing on informed decision making. To further capitalize on the platform provided by PDAs, they could combined with predictive models, similar to the previously discussed DSS, and provide individualized outcome predictions. PDA's would be the patient version of the DSS and could stand to benefit from a link between these two platforms. Using the DSS, the clinician could select patient specific treatment options (e.g. treatments for which the patient is eligible) and use the clinical, disease and treatment parameters to calculate personalized outcome probabilities (Figure 8.2). This information could then be presented to the patient through the interface of the PDA in an understandable and interpretable manner. This could improve the effectiveness of patient-doctor consults as well as better inform the patient and empower him in the decision making process without confusing him or providing him with non-applicable information. 
This presents two different challenges for this to be achieved: how to communicate the results of the DSS to the patient clearly without possible misinterpretation, and how to take into account patient preferences to affect the DSS results. The results of a DSS have large uncertainties and the models incorporated are often based on clinical trials, which may not be representative for the patient. A medical professional has the skills and knowledge to properly interpret these results and apply them accordingly, but a patient might not. This is why it is crucial to consider how to present these results to a patient. A possible solution could be to indicate a risk level for certain comorbidities or cancer free survival, rather than exact risk levels.

The second challenge arises due to the way the impact on the quality of life a certain comorbidity has, expressed in utility values, is averaged over a population, and thus might not reflect a specific patient. A possible solution to this issue could be to allow the patient to indicate impact of certain comorbidities on his perceived quality of life, and scale the utility values accordingly.

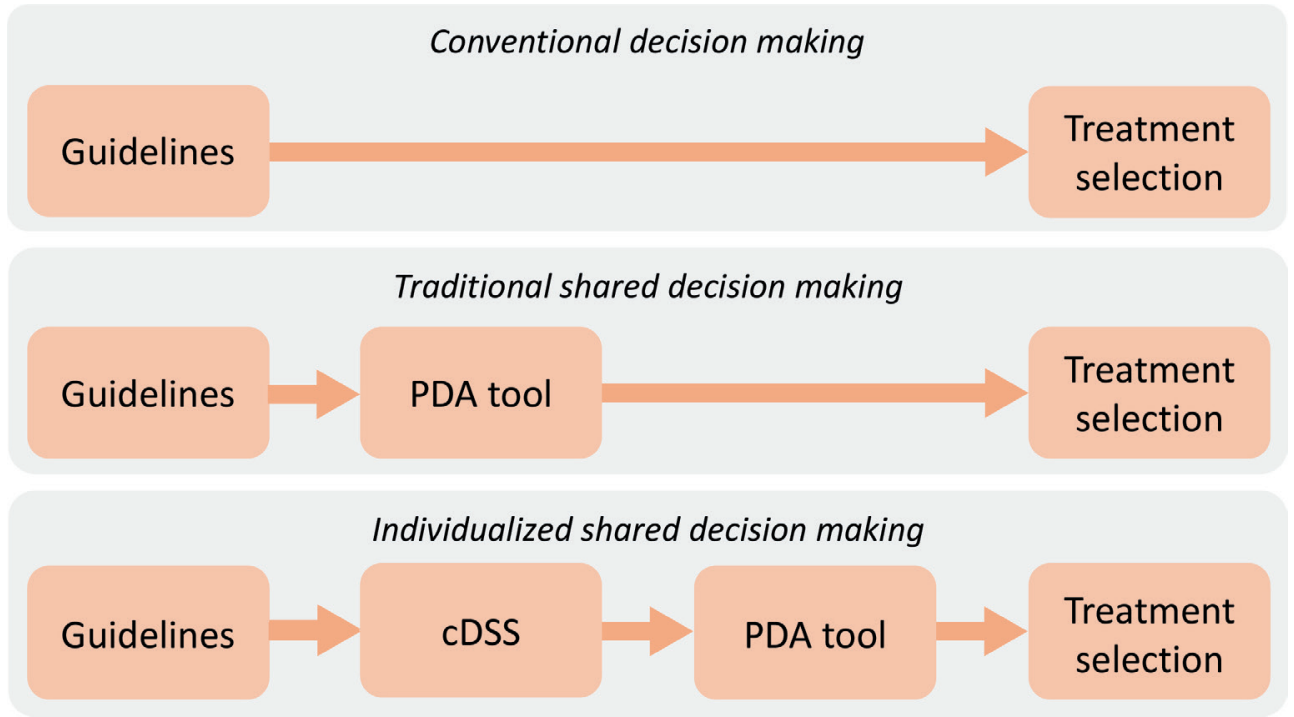

Figure 8.2 - Different types of decision making. PDA: Patient decision aid; DSS: clinical decision support system 


\section{Concluding message}

The aim of this thesis was to bridge the gap between machine learning based models and the decision making process through the development of DSS for prostate cancer patients. We made the first steps towards meeting this aim by developing two new DSS. We discussed different challenges and methods to develop and apply these DSS and challenged their robustness and clinical benefit. The results implicate that the application of these DSS has the potential to increase effectivity and reduce treatment related toxicity. Although the models and frameworks presented require external validation, expansion and refinement before being ready for clinical practice, this work facilitates some important steps towards bringing the gap between machine learning models towards tailored treatment selection for prostate cancer patients. 


\section{References}

1. Michalski, J.M., et al., Effect of Standard vs Dose-Escalated Radiation Therapy for Patients With Intermediate-Risk Prostate Cancer: The NRG Oncology RTOG 0126 Randomized Clinical Trial. JAMA Oncol, 2018. 4(6): p. e180039.

2. Karsh, L.I., et al., Absorbable Hydrogel Spacer Use in Prostate Radiotherapy: A Comprehensive Review of Phase 3 Clinical Trial Published Data. Urology, 2018. 115: p. 39-44.

3. Vanneste, B.G., et al., Spacers in radiotherapy treatment of prostate cancer: is reduction of toxicity cost-effective? Radiother Oncol, 2015. 114(2): p. 276-81.

4. Vanneste, B.G., et al., Who will benefit most from hydrogel rectum spacer implantation in prostate cancer radiotherapy? A model-based approach for patient selection. Radiother Oncol, 2016. 121(1): p. 118-123.

5. Michalski, J.M., et al., Radiation dose-volume effects in radiation-induced rectal injury. Int J Radiat Oncol Biol Phys, 2010. 76(3 Suppl): p. S123-9.

6. Appelt, A.L. and I.R. Vogelius, A method to adjust radiation dose-response relationships for clinical risk factors. Radiother Oncol, 2012. 102(3): p. 352-4.

7. Carneiro, A., et al., Are localized prostate cancer biomarkers useful in the clinical practice? Tumour Biol, 2018. 40(9): p. 1010428318799255.

8. Narayan, V.M., B.R. Konety, and C. Warlick, Novel biomarkers for prostate cancer: An evidence-based review for use in clinical practice. Int J Urol, 2017. 24(5): p. 352-360.

9. Sachdev, S., et al., Assessment of Postprostatectomy Radiotherapy as Adjuvant or Salvage Therapy in Patients With Prostate Cancer: A Systematic Review. JAMA Oncol, 2020.

10. Moons, K.G., et al., Critical appraisal and data extraction for systematic reviews of prediction modelling studies: the CHARMS checklist. PLoS Med, 2014. 11(10): p. e1001744.

11. Moons, K.G.M., et al., PROBAST: A Tool to Assess Risk of Bias and Applicability of Prediction Model Studies: Explanation and Elaboration. Ann Intern Med, 2019. 170(1): p. W1-W33.

12. Goncalves, A., et al., Generation and evaluation of synthetic patient data. BMC Med Res Methodol, 2020. 20(1): p. 108.

13. Pollack, A.H., et al., Creating synthetic patient data to support the design and evaluation of novel health information technology. J Biomed Inform, 2019. 95: p. 103201.

14. Hooshangnejad, H., et al., FEMOSSA: Patient-specific Finite Element Simulation of the Prostate-Rectum Spacer Placement, a Predictive Model for Prostate Cancer Radiotherapy. Med Phys, 2021.

15. Hamstra, D.A., et al., Sexual quality of life following prostate intensity modulated radiation therapy (IMRT) with a rectal/prostate spacer: Secondary analysis of a phase 3 trial. Pract Radiat Oncol, 2018. 8(1): p. e7-e15.

16. Nawa, K., et al., Evaluation of a commercial automatic treatment planning system for prostate cancers. Med Dosim, 2017. 42(3): p. 203-209.

17. Cilla, S., et al., Template-based automation of treatment planning in advanced radiotherapy: a comprehensive dosimetric and clinical evaluation. Sci Rep, 2020. 10(1): p. 423.

18. Goy, B.W., et al., Ten-Year Treatment Outcomes of Radical Prostatectomy Vs External Beam Radiation Therapy Vs Brachytherapy for 1503 Patients With Intermediate-risk Prostate Cancer. Urology, 2020. 136: p. 180-189.

19. Goy, B.W., et al., Treatment results of brachytherapy vs. external beam radiation therapy for intermediate-risk prostate cancer with 10-year followup. Brachytherapy, 2016. 15(6): p. 687-694.

20. Nagore, G., et al., High dose rate brachytherapy for prostate cancer: A prospective toxicity evaluation of a one day 
schedule including two 13.5Gy fractions. Radiother Oncol, 2018. 127(2): p. 219-224.

21. Guimas, V., et al., Focal or whole-gland salvage prostate brachytherapy with iodine seeds with or without a rectal spacer for postradiotherapy local failure: How best to spare the rectum? Brachytherapy, 2016. 15(4): p. 406-411.

22. Mahal, B.A., et al., Use of a rectal spacer with low-dose-rate brachytherapy for treatment of prostate cancer in previously irradiated patients: Initial experience and short-term results. Brachytherapy, 2014. 13(5): p. 442-9.

23. Choyke, P.L. and S. Loeb, Active Surveillance of Prostate Cancer. Oncology (Williston Park), 2017. 31(1): p. 67-70.

24. Hayes, J.H., et al., Active surveillance compared with initial treatment for men with low-risk prostate cancer: a decision analysis. JAMA, 2010. 304(21): p. 2373-80.

25. D’Agostino, M., et al., Open data and public health. Rev Panam Salud Publica, 2018. 42: p. e66.

26. Wilkinson, M.D., et al., The FAIR Guiding Principles for scientific data management and stewardship. Sci Data, 2016. 3: p. 160018.

27. Grol, R. and M. Wensing, What drives change? Barriers to and incentives for achieving evidence-based practice. Med J Aust, 2004. 180(S6): p. S57-60.

28. Vanneste, B.G., et al., Prostate Cancer Radiation Therapy: What Do Clinicians Have to Know? Biomed Res Int, 2016. 2016: p. 6829875.

29. Clayman, M.L., P. Gulbrandsen, and M.A. Morris, A patient in the clinic; a person in the world. Why shared decision making needs to center on the person rather than the medical encounter. Patient Educ Couns, 2017. 100(3): p. 600-604.

30. Charles, C., A. Gafni, and T. Whelan, Shared decision-making in the medical encounter: what does it mean? (or it takes at least two to tango). Soc Sci Med, 1997. 44(5): p. 681-92.

31. Lamers, R.E., et al., Patients are dissatisfied with information provision: perceived information provision and quality of life in prostate cancer patients. Psychooncology, 2016. 25(6): p. 633-40.

32. Sciarra, A., V. Gentile, and V. Panebianco, Multidisciplinary management of Prostate Cancer: how and why. Am J Clin Exp Urol, 2013. 1(1): p. 12-7.

33. Davison, B.J. and L.F. Degner, Empowerment of men newly diagnosed with prostate cancer. Cancer Nurs, 1997. 20(3): p. 187-96.

34. Lin, G.A., et al., Patient decision aids for prostate cancer treatment: a systematic review of the literature. CA Cancer J Clin, 2009. 59(6): p. 379-90.

35. Ankolekar, A., et al., Development and validation of a patient decision aid for prostate Cancer therapy: from paternalistic towards participative shared decision making. BMC Med Inform Decis Mak, 2019. 19(1): p. 130. 

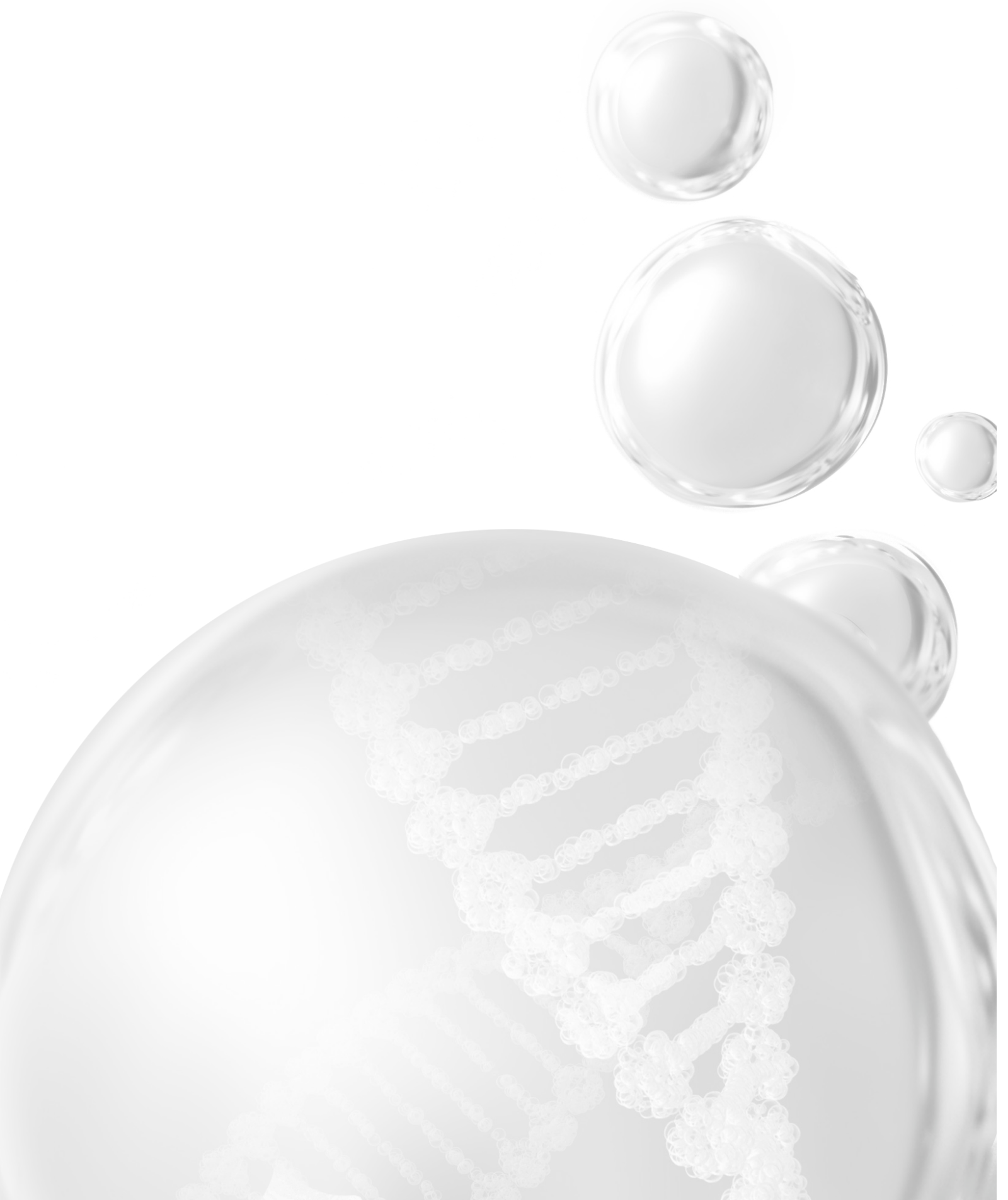
Appendices 

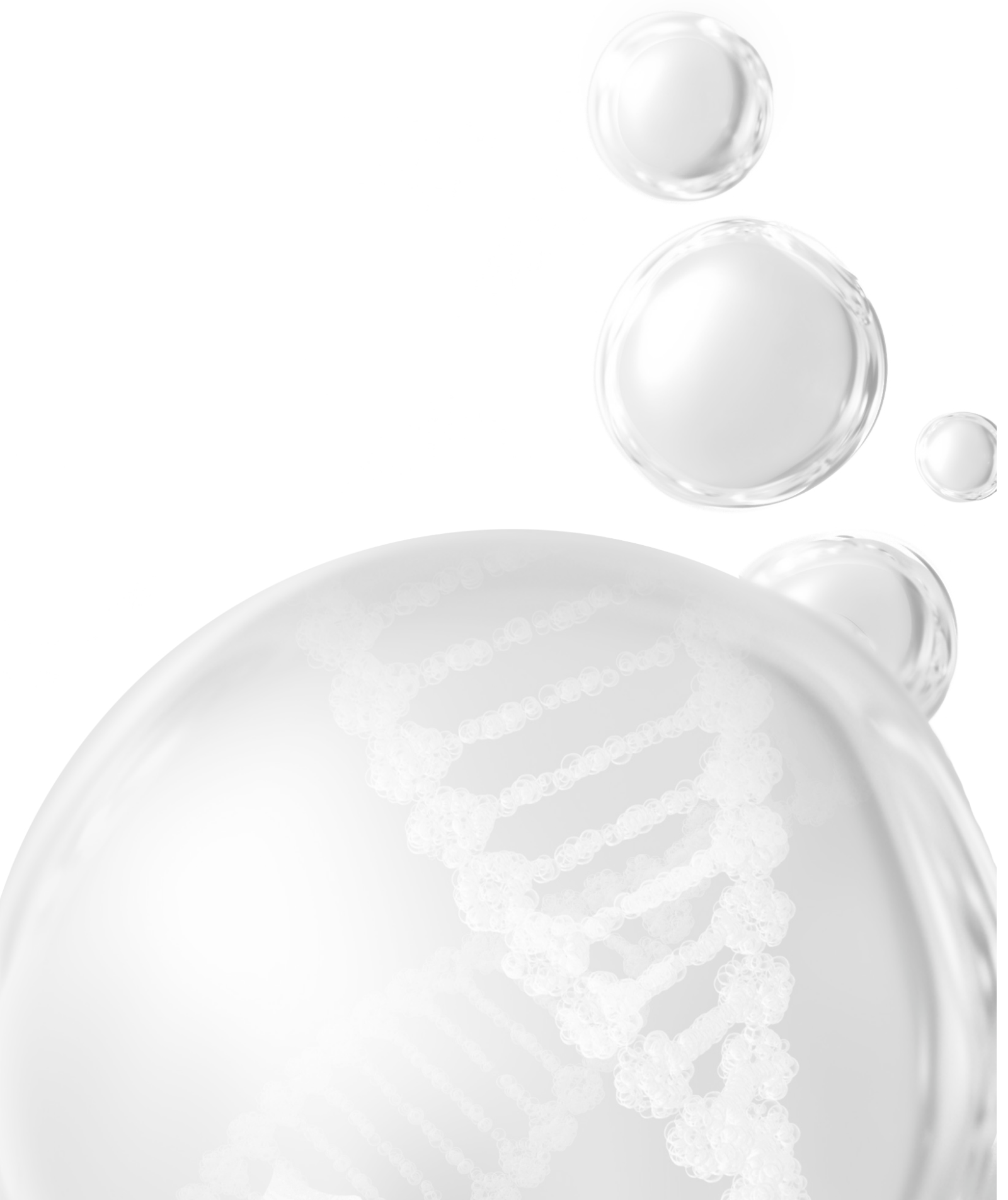
Summary 
Prostate cancer (PCa) is the second most commonly diagnosed cancer for men, and therefor forms a topic of significant research interest. Especially lowintermediate risk PCa, which forms $40 \%$ of the newly diagnosed PCa, has a variety of viable treatment options. The options include passive treatments such as active surveillance, and active treatments such as radiotherapy and prostatectomy. Within treatment types there are still a number of options, such as within radiotherapy the choice exists between brachytherapy or external beam radiotherapy (EBRT), and additional options, such as hormonal therapy or rectum sparing devices can be considered. This, combined with an increasing number of biomarkers make that PCa is an excellent candidate for improvement via personalized medicine.

The importance of personalized medicine has become progressively evident over the past few years, focusing on patient variations, outcome predictions and patient preferences. An important step towards personalized PCa treatment would be a clinical decision support system (DSS), that aids optimizing treatment selection. DSS are typically software applications such as recursive partitioning analyses models, nomograms or websites such as https://www.ai4cancer.ai. A DSS is used to support the groups responsible for deciding on patient treatment, such as the physician, the tumor board and the patient, in making a knowledgeable decision regarding treatment options. The development of such DSS was the primary focus of this thesis.

In Chapter 3 we provided an overview of existing literature on the topic of DSS in the field of PCa. It was found that a large number of diagnosis and staging DSS are becoming available as well as tools that improve cancer detection, predicting treatment outcome and outcome stratifications. However, limited DSS for treatment selection were found in this literature study, which were mostly centered around EBRT. A lack of DSS for other treatment modalities suggests that the development of new tools are necessary to compare objectively different treatment modalities. Additionally, the field of patient informed decision-making is still in its infancy, but essential for the growth towards individualized medicine.

Other chapters in this work focus on developing, improving and challenging treatment DSS both within the field of EBRT, as well as interdisciplinary comparisons.

Chapter 4 describes the development of a Virtual Implantable Rectum Spacer (V-IRS) that can be used to support the decision for the implantation of an implantable rectum spacer (IRS) for a specified patient or not. An IRS is a device (such as a hydrogel or a biodegradable balloon implant) that is placed between the anorectum wall and the prostate prior to the start of EBRT. This device spares the rectum from high dose levels and reduces the risk of long term treatment related side-effects such as bleeding. The V-IRS uses image deformation on a CT 
scan of a patient to render a virtual CT scan of the same patient with a V-IRS. The advantage of this method is that a CT of a patient with an IRS can be generated and made available for treatment planning purposes without the real implantation of the IRS itself. Moreover, we used this method to develop a DSS that, instead of only looking at the improved dose reduction by implanting the IRS, also calculated the toxicity risk reduction and the cost-effectiveness (CE) for comparison. This allows for a decision based not only on dose reduction, but also on the health gain and the costs associated with the health gain. We tested this V-IRS in the following manner: we generated virtual CT scans and performed dose planning on these. We then calculated relevant dose metrics, toxicity risk and performed a CE analysis. This was repeated on CT scans of the same patients with a real IRS, and the results were compared to those of the virtual CTS. The V-IRS resulted in the same classification (place an IRS or don't place an IRS) as the real IRS.

In this study we found that the implantation of an IRS is not cost-effective for all patients, but does provide significant benefit for some, so individual patient assessment could improve the quality of care. We concluded that the V-IRS approach in combination with a toxicity model and a CE analysis can serve as the basis for a decision support system for the implantation of either a hydrogel IRS or a rectum balloon implant.

To expand on the DSS described in the previous chapter, in Chapter $\mathbf{5}$ we developed an isotoxic method integrating genetic markers of rectal radio-sensitivity combined to integrate into this DSS. The method calculates the maximum dose per fraction that can be given without exceeding an upfront determined limit for the risk of toxicity, rather than calculating the risk of toxicity resulting from a given dose.

The results show that a higher prescribed dose should be given to improve tumor control, and can be safely administered when combined with an IRS.

The isotoxic model integrating genetic markers for rectal toxicity developed in this study can be used to evaluate a treatment plan, and test how much dose can be given without causing excessive damage to the rectum. Thus sparing the organs at risk at a chosen described level while optimizing the TCP. In combination with the V-IRS, this method can serve as the basis for a DSS for the implantation of an IRS.

One type of IRS is the saline-filled biodegradable balloon implant. Chapter 6 provides an evaluation of the balloon volume stability that is based on weekly cone-beam CT measurements during the full course of EBRT, because a RBI volume decrease can be expected over time. In this study, we analyzed the dosimetric consequences of this phenomenon and predicted the increase in risk of late rectal bleeding (LRB) resulting from a shrinking balloon implant to assess its potential clinical impact. We concluded that despite that the weekly RBI shrinkage was significant, 
neither significant increase in absolute volume or the anorectum receiving at least 75 Gy, nor in predicted LRB risk were observed over the full treatment course. Only when the prostate-rectum distance decreased to under $1 \mathrm{~cm}$ a treatment plan adaptation would be advisable. The advantage of this work with relation to the previous chapters is that it shows that the exact shape of the V-IRS is not crucial to proper classification considering treatment advice. This is important as the exact shape and position of both the anorectum and the IRS are difficult to accurately predict from a single CT scan.

In Chapter 7 we developed an interdisciplinary DSS, which would aid in the treatment selection of either EBRT or radical prostatectomy (RP) and tested this on a synthetic patient dataset. We validated the DSS against published clinical studies and set up an in silico trial for patients between 75 and 80, eligible for both RP and EBRT. We also assessed the CE of a treatment allotment strategy based on the DSS compared to a randomized treatment allotment strategy. Our first hypothesis was that we could accurately replicate results from published studies, which we aimed to confirm by generating synthetic datasets with clinical parameters similar to published trials. The DSS largely replicated the published results accurately. The relative differences between the treatment modalities and fractionation plans were replicated by the model, and the conclusions of the DSS and the studies agreed. We also performed an in silico trial using the DSS, exclusively including elderly patients both without and with prior ED and found that for the first group EBRT was preferred, and for the second RP performed better in terms of QALYS. Additionally, we hypothesized that a treatment selection strategy based on the DSS would improve tumor control, reduce toxicity, and improve CE as opposed to randomized treatment selection. Our CE analyses suggest that not only do the costs of treatment decrease with the application of a DSS, but the number of QALYS also increases, making the integration of a DSS dominantly cost-effective compared to current clinical practice. The expected cost savings within the Netherlands when using a DSS could be as high as EUR 3.8 million fover five years, assuming 2400 patients are affected every year. Additionally, the number of patients with recurrence after treatment could be reduced slightly by $2 \%$.

This chapter lays the groundwork for a detailed, personalized treatment DSS that aids in the choice between EBRT and RP for low to intermediate risk PCa patients. This DSS could be used for in silico clinical trials when applied to a synthetic dataset, which would be a valuable precursor to clinical trials.

Chapter $\mathbf{8}$ provides a general discussion and future perspectives, which is closed with a concluding message. It is discussed that one strength of this thesis is that it lays the groundwork for an extensive DSS for treatment selection for PCa. A variety of methods is presented in this work including the iso-toxic method, incorporating 
new biomarkers into existing models, the use of synthetic patient datasets, and in silico clinical trials, all of which could aid in the development of future DSS.

Future works should include the improvement, optimization and validation of the DSS developed in this thesis. It is also advisable to combine the IRS DSS and the EBRT versus RP DSS into a single framework and expand this with other treatment modalities such as watchful waiting and brachytherapy. Finally, the next step towards personalized treatment for PCa would be to combine the DSS framework with a patient decision aid, so that patient preferences would provide valuable input to the DSS and the DSS could be used to customize information for the patient decision aid. 

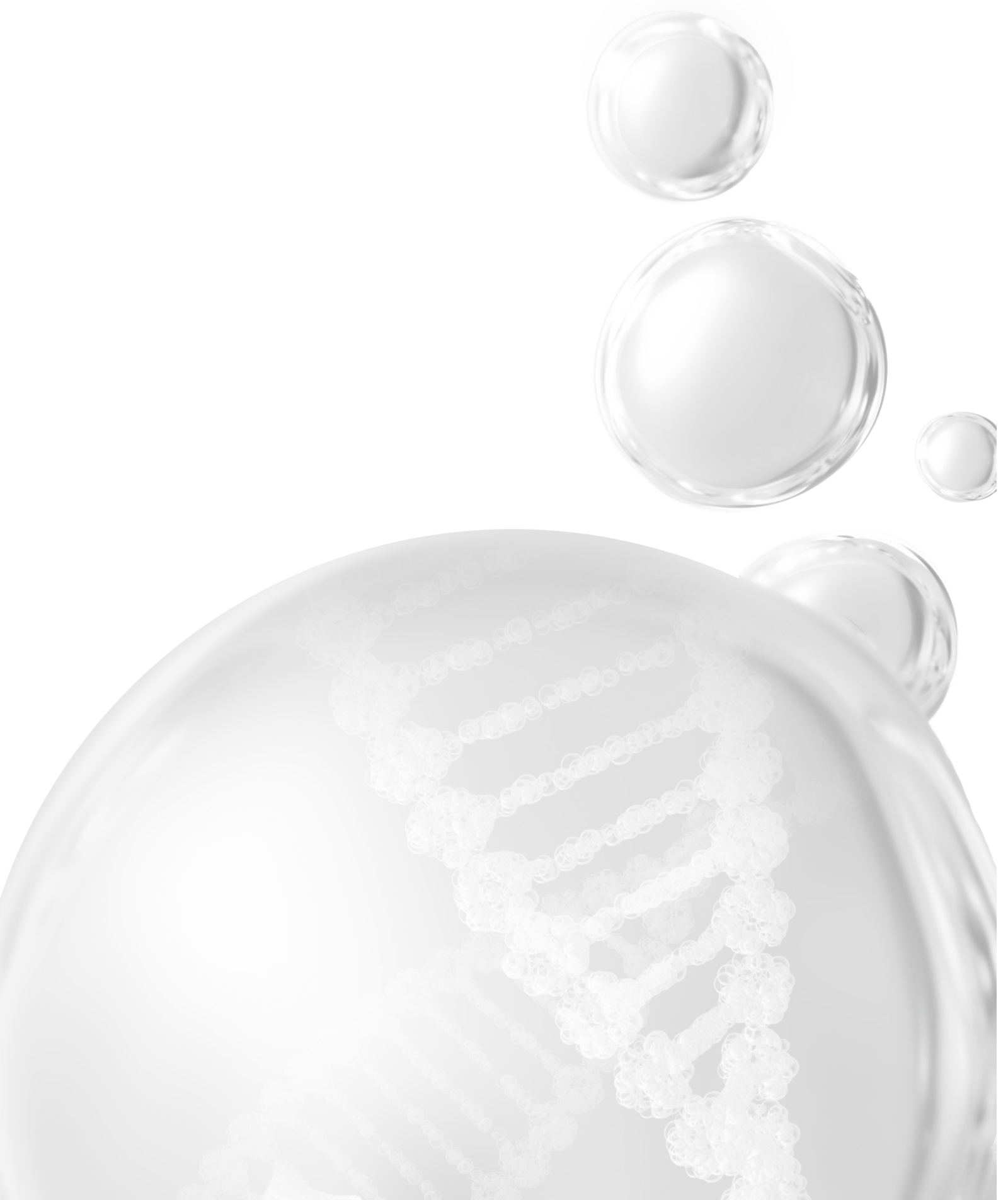


\section{Impact Paragraph}




\section{Social relevance and target groups}

Prostate cancer is a very common cancer type, and although mortality rates are low, it touches the lives of a large number of men. This disease has a range of viable treatment options and a large number of clinical parameters and biomarkers predictive for the primary outcome of these treatments, as well as treatment related side effects. Ideally these factors should therefore guide optimized treatment selection. In practice, however, this is rarely applied and treatment selection is often based on factors largely unrelated to the patients clinical characteristics and treatment outcomes.

Both previously published studies and the research done in this thesis suggest that the quality of life of prostate cancer patients has the potential to be improved through personalized treatment selection. This, combined with the large number of patients diagnosed every year, effectuates optimized treatment selection would have large social impact.

The primary aim of this thesis was to combine existing models predictive of treatment outcome and published biomarkers into clinical decision support systems in order to bridge the gap between literature and clinical practice. This has as an advantage that the large number of clinical factors such as disease parameters, treatment parameters, patient characteristics and genetic markers could be condensed into an easy to handle number of factors, such as expected cure rate, risk of treatment related side-effects and cost-effectiveness. Since the human cognitive ability is limited, and only a number attributes can be recalled during decision making, this condensation of information would streamline the decision making process. Therefore not only the patient is ultimately benefited by the application of such tools, but clinical professionals will be empowered during treatment selection as well.

\section{Primary findings and products}

The primary output of the work presented in this thesis are the methodologies and algorithms that form the basis of the developed decision support systems. All the studies published in this thesis suggest that the application of these models in a clinical environment has the potential to improve the quality of life of patients, often providing monetary value, which underscores the advantage of the developed decision support systems.

The methodologies have been published and described in great detail, sufficient for anyone with access to the publications to replicate the work that was done. This makes it possible for the methods used to be applied in other projects, either for different ends or to follow-up the work that was published.

In addition to the descriptions of model parameters and methods, the code written to implement the decision support systems and generate the results presented in 
this thesis were made available on GitHub, so that they may be applied, reused, adapted and improved in future projects. The models as a whole will also be made available on a public platform currently under construction.

\section{Innovation}

The platform that forms the basis of all the decision support systems presented in this thesis incorporate different models and combine them in a single application. Models eligible for integration are those that predict outcome or toxicity, dose metrics or cost-utility. From a scientific point of view this is beneficial as the methods described combine separate instances of previously published models and biomarkers and provides a bridge for them to be directly applied towards the benefit of the clinical professional and the patient. This improves the prospects with respect to the usefulness and impact of valuable scientific research. To our knowledge, our methods are the first within the field of prostate cancer that work to actively selecting, refining and adapting existing knowledge towards clinical benefit in such a manner.

As mentioned before, the methodologies and code have been made available for further use. Especially the Markov model described in Chapter 7 was developed and described in great detail, including transition probabilities to a large number of health states and detailed descriptions of treatment costs. This information can prove invaluable for other researchers looking to build a cost-effectiveness model concerning the primary treatment of prostate cancer, as many of these model parameters can be recycled, which would streamline and simplify this part of the work for future researchers.

The method for generating synthetic patients is an emerging field, and has to potential to be useful to validate newly developed models against published clinical trials, even when available patient and treatment parameters are incomplete, such as was done in Chapter 7. This allows for relatively cheap and fast testing of models prior to (and potentially to inform) setting up expensive and time consuming retrospective or prospective clinical trials. Similarly, the in silico trial is a possible application of the synthetic patient datasets. An in silico trial could be used to test new methods or technologies, as well as to gain insight into underrepresented patient populations. The information gained from this could be used to better prepare prospective or retrospective clinical trials prior to investing the time and expenses required of such a project. This could result in better designed clinical trials and possibly preventing waste in the form of effort, time and money.

\section{Implementation}

As discussed in the general discussion of this thesis, the developed decision support systems require improvement, optimization, expansion and (prospective) 
validation before further steps can be made toward introducing them into clinical practice. Projects have already been set up for these developments, one of which with the particular aim of including active surveillance into the framework and validation on a patient cohort through a clinical trial. In parallel, work is being done to adapt the framework presented here into a personalized patient decision aid.

When these important developments have taken place, the framework could be properly developed into a software application that would be suitable for commercial use. This development should take place with close cooperation between the developing party (such as a company or a research group) and the potential end users (clinical professionals). This final product could then be subjected to regulatory bodies and presented to hospitals and clinics. This would make the technologies developed in this work available to the end user (the medical professional) and the target group (prostate cancer patients) and potentially improve the quality of life for this patient population. 
Acknowledgements 
As I am putting the finishing touches on my dissertation, I am looking upon my work with no small degree of satisfaction. It has been a long road, and by no means an easy one, but I am happy I took it. This reflection brings to mind the large group of people who aided in my achievements, helped pave this road or ease the way for me, and this chapter provides me with the perfect opportunity to acknowledge these people and thank them.

One of the key players in my work the past years has been my promotor, professor Philippe Lambin. Not only was he the catalysator of my PhD, with brilliant ideas and opening up opportunities such as conferences, courses and collaborations, he also displayed a large amount of faith in me. His way of thinking inspired me, his opportunities helped me grow as a professional, and his faith and support in me helped me grow from an unsure student to a proper researcher. Thank you for all you have done for me over the years, your impact on me will echo through the rest of my career.

Philippe was not alone in supporting me during my PhD, however, and I would like to take this opportunity to thank my co-promotors, dr. Bram Ramaekers and dr. Ben Vanneste. These brilliant minds provided the perfect balance that my thesis needed: on one side the medical expertise and experience of a radiologist, and on the other side the technical input and guidance from an economic modelling expert. Without the input from either, presenting the machine learning and economic modelling based decision support systems in a medically relevant way would have been difficult. Every abstract, every figure, every paper was thoroughly subjected to their excellent feedback, which they provided with an excruciating eye for detail. They helped raise the quality of this work to its current state, and for this I am grateful.

This brings me to the next group of people I would like to thank for improving the quality of this thesis: my assessment committee. Thank you, professor Frank Verhaegen, professor David Azria, professor Marco van Vulpen and dr. Ben Wijnen for taking the time to critically read my thesis and providing me with valuable feedback.

Next I would like to bring to light some important collaborations with valuable colleagues, which helped me shape my work into its final form. Dr. Seán Walsh provided me with some much needed guidance during the start of my PhD years, and helped me incorporate one of his models into my own decision support system. So thank you very much for the pleasant welcome. Later, the task of gently directing me along the correct course of my PhD fell upon dr. Cary Oberije. Thank you, Cary, for helping me through some tough challenges and for the pleasant trip we shared to MD Anderson. Another strong influence on my work through supervision, brainstorming sessions and general expertise was dr. Henry Woodruff, who helped me in writing one of my toughest papers during one of the more difficult years of my PhD. Thank you for your patience and constant support.

I am also very grateful to some of the key contributors to my work. Iva Halilaj strongly supported me in writing one of my papers, dr. Wouter van Elmpt and professor André 
Dekker provided me with early inspirations and idea's for my first paper, dr. Arthur Jochems who helped ensure the quality of my coding, and all other co-authors who provided valuable feedback to my work over the years.

For a large project I really enjoyed working together with dr. Marc Lobbes. This collaboration was really insightful to me, especially since this gave me the opportunity to see the patients we were trying to help. Together with dr. Henry Woodruff, Yousif Widaatalla, Renée Granzier and Manon Beuque, this was a great team to be a part of.

The contribution of two people cannot be overstated when considering the logistics of publishing papers, submitting for grants, attending conferences and obtaining a PhD: Rianne Herben and Floor Franssen. For any and all questions relating to these matters, these two people were sources of information, help and effort, and for this I extend my sincere thanks to them.

Aside from professional collaborations and input, a pleasant work environment can make the challenging years one spends on obtaining a PhD a satisfying and enriching experience. The group of people I found myself working with changed over the years, but was constantly agreeable, welcoming and engaging. For me it was often exhausting to make the trip between Eindhoven and Maastricht, but luckily I had this team of colleagues who were worth the trip. Evelyn, you were one of the first people who welcomed me to the D-lab and made me feel comfortable as a colleague, and your kind and empathetic character is something I was very grateful for. Iva, thank you for being my friend and being such excellent company during the second half of my PhD. I really enjoyed being roommates with you and spending time with you outside of work! Relinde, Elizaveta and Martha, my other roommates, thank you for making the trip to Maastricht worth it, I already miss sharing a room with you. Sergey, Yousif, Esma and Henry I would in particular like to thank for the pleasant conversations during parties and coffee breaks and lunches. Cary, Timo, Ralph and Janita, thanks for making my first two trips abroad for work a lot easier and less daunting, you were great traveling companions. And of course a big thanks to all my other colleagues who have come and gone and made these past few years as pleasant as they were: Abdalla, Alberto, Aniek, Arthur, Avishek, Brent, Cecile, Daniela, Esther, Fadila, Giacomo, Guangyao, José, Jurgen, Manon, Karen, Renée, Ruben, Sebastiaan, Sebastian, Simon, Simone, Sithin, Timo, Turkey, William, and anyone else who has worked with me these past years.

Professional support from supervisors, collaborators and colleagues is invaluable during the completion of a PhD, but the support I felt from the personal side of my life cannot be overstated. For me, this emotional support, either in the form of good advice, interest in my work or relaxation has proven to be of significant worth, and I would like to take the time to thank the people who provided said support. 
First I would like to thank my closest friends, with whom I have spent hours in conversation, and who always offered a listening ear when I needed to get something off my chest. Michiel, Jenny, Iva and Rob were my most frequent victims, and will probably remain so for many years to come. Thanks for always being there. Also a special shout-out to Rob, Erik, Garm, Frank and Tamara, who have suffered me venting my frustration into videogames with them, and who tolerated my worst side with remarkable grace.

This brings me closer to home, namely to family. I am blessed with immense luck that I am close to my family, both relationship-wise and location-wise. All of them, both my blood and my family-in-law have been understanding and supportive of my work, which is always deeply appreciated. Maria and Willy I would like to thank for the advice that was often given as well as the understanding when I would be writing code on family vacations. Dankjewel aan opa en oma van Wijk en opa Klerks dat jullie altijd interesse toonden in waar ik mee bezig was, en jullie liefde en steun vrijelijk aan hebben geboden deze jaren.

Tamara, you played a very unique roll during my PhD. As my only sister, it was naturally unavoidable that I would always talk to you about every little thing going on in my life, so you have my thanks for listening and understanding. But more than that you were always my friend, and you spent effort and time on me that you are by no means obligated to do through our familial bond. On top of that, you aided me in designing this book through your professional expertise as a graphic designer. So not only do I thank you as my family and my friend, but also as a colleague.

And now we come to some of the most crucial people when it came to shaping me both as a person and as a professional: my mom and dad, Rens and Henri. My father, who I have looked up to, competed against and respected as an academic and professional, was one of the biggest reasons for me to pursue a PhD to begin with. Even when I struggled, he taught me to work harder and push through, and to always ask for help when needed. My mother provided an environment that oozed comfort and support whenever I studied. I have fond memories of studying for exams as she kept me foreseen of a steady stream of coffee and snacks. Both my parents have provided me with mountainous support during my PhD, and I can't thank them enough.

Finally I come to my husband, Frank. Words cannot express my gratitude, and words are not needed. 

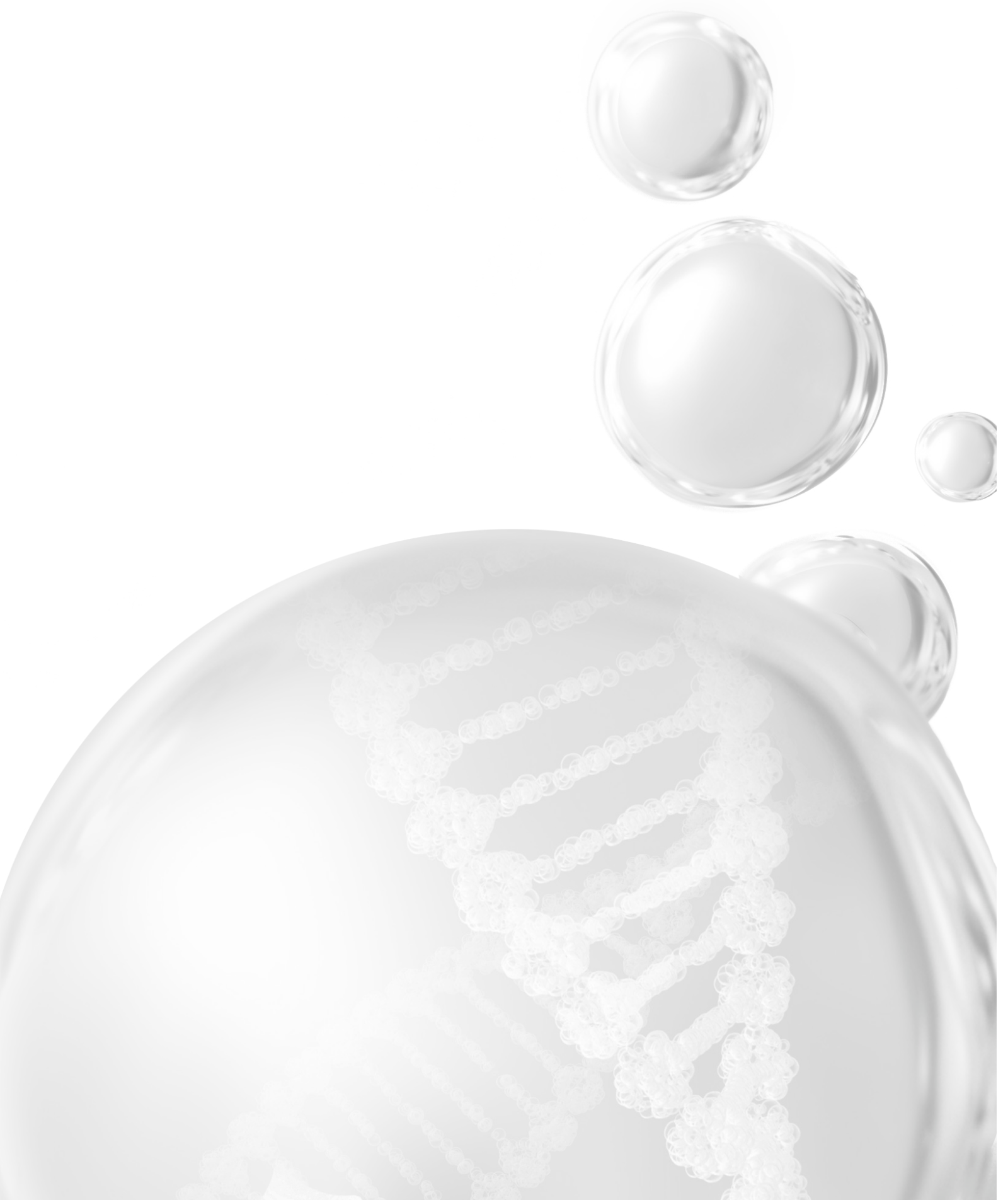
Curriculum vitae 


\section{Curriculum Vitae}

\section{Personal Information}

\begin{tabular}{l|l} 
Name: & Yvonka van Wijk \\
Date of Birth: & November 9th 1989 \\
Nationality: & Dutch \\
Phone number: & $+31(0) 681919788$ \\
E-mail address: & yvonkavwijk@gmail.com
\end{tabular}

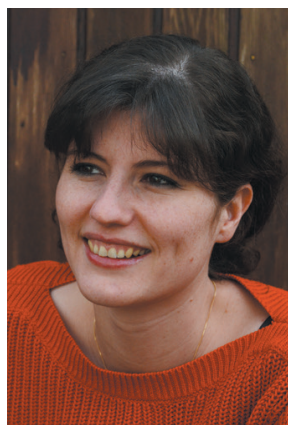

\section{Characteristics}

» Team player: I work well within multidisciplinary groups, and am able to show initiative

» Empathic: I can relate to others, which helps during cooperative work.

» Passionate: I care about my work and feel energized by my achievements.

» Focused: I will always try to deliver quality work without losing sight of the bigger picture

Education

2016-2021

2013-2015

PhD Student at Department of Radiotherapy (Precision Medicine, D-lab)

University of

Thesis subject: Development of decision support tools for

Maastricht prostate cancer patients, focusing on radiation therapy.

Master Biomedical engineering

Eindhoven University of Thesis: "Treatment planning for Microwave ablation of Technology hepatic tumors in the proximity of hepatic veins."

2008-2013

Bachelor Biomedical engineering

Eindhoven University of Thesis: "Simulation of Ultrasound in Bone and Muscle using Ray-tracing"

Practical Experience

\begin{tabular}{l|ll} 
2020-NOW & $\begin{array}{l}\text { Validation Lead at the Interventional Applications Cluster } \\
\text { 2020W }\end{array}$ & $\begin{array}{l}\text { Employeneur at TMC Data Science } \\
\text { Responsibilities: } \\
\text { Personal development; Contributing to the business; } \\
\text { Professional Socializing; Furthering the business }\end{array}$ \\
$\mathbf{2 0 1 6 - 2 0 2 1}$ & $\begin{array}{l}\text { PhD Student at Department of Radiotherapy (Precision } \\
\text { Medicine, D-lab) } \\
\text { Responsibilities: } \\
\text { Developing models; Conducting research; Writing } \\
\text { papers; Writing grants; Supervising master students }\end{array}$ & TMC \\
\end{tabular}




\begin{tabular}{l|ll}
$\mathbf{2 0 1 7 - 2 0 2 0}$ & $\begin{array}{l}\text { Consultant for ptTheragnostic } \\
\text { Responsibilities: } \\
\text { CE marking; Developing models; Preparing and giving }\end{array}$ & \\
demos; Giving pitches & DNAmito \\
$\mathbf{2 0 1 3}$ & $\begin{array}{l}\text { Master Thesis Internship } \\
\text { Responsibilities: } \\
\text { Developing models; Conducting research; Writing Thesis }\end{array}$ & Philips Research \\
& $\begin{array}{l}\text { Master Internship } \\
\text { Responsibilities: } \\
\text { Developing models; Conducting research; Writing Thesis }\end{array}$ &
\end{tabular}

\section{Skills}

\section{Languages}

\begin{tabular}{l|ll} 
& \multicolumn{1}{c}{ Spoken } & Written \\
\hline Dutch & excellent & excellent \\
English & excellent & excellent \\
German & moderate & moderate
\end{tabular}

Software Skills

\begin{tabular}{l|ll}
\multicolumn{2}{l}{ Description } & Level of mastery \\
\hline Matlab & arithmetic program & excellent \\
Comsol Multiphysics & Multiphysics FEM package & excellent \\
Wolfram Mathematica & computation package & good \\
Python & programming language & moderate \\
$R$ & Computation package & good
\end{tabular}

\section{General Skills}

\begin{tabular}{l|ll} 
& \multicolumn{1}{l}{ Experience } & Level of mastery \\
\hline Classification (Random forest) & Hands on & excellent \\
Radiomics & Hands on & excellent \\
CE modelling & PhD courses & excellent \\
Regression & Master courses, hands on & good \\
Signal analyses & Master courses & good \\
Statistics & Master courses & good \\
Image analyses & Internships, courses & good
\end{tabular}




\section{Courses/Congresses}

\begin{tabular}{l|ll}
$\mathbf{2 0 1 9}$ & CE modelling in R & Maastricht University \\
$\mathbf{2 0 1 9}$ & European Congress of Radiology & Vienna, Austria \\
$\mathbf{2 0 1 8}$ & EUSOBI 2018 & Athens, Greece \\
$\mathbf{2 0 1 8}$ & PhD Writing 2 & Maastricht University \\
$\mathbf{2 0 1 8}$ & Presentations Course for PhD Researchers (PhD-4) & Maastricht University \\
$\mathbf{2 0 1 7}$ & Prediction and Modeling of response to Molecular and & Cancéropôle Grand Ouest \\
$\mathbf{2 0 1 7}$ & External Beam Radiotherapies & \\
$\mathbf{2 0 1 6}$ & BiGART 2017 & Aarhus, Denmark \\
$\mathbf{2 0 1 6}$ & PhD Writing 1 & Maastricht University \\
$\mathbf{2 0 1 6}$ & Antroduction modelling cost-effectiveness & Erasmus Academie
\end{tabular}

\section{Publications}

"Modeling-Based Decision Support System for Radical

"Decision Support Systems in Prostate Cancer Treatment: An Overview."

"Decision support systems in oncology"

"Development of an isotoxic decision support system integrating genetic markers of toxicity for the implantation of a rectum spacer."

"Big Data-Based Decision Support Systems for Hadron Therapy" (book chapter)

"Dynamics of rectal balloon implant shrinkage in prostate VMAT : Influence on anorectal dose and late rectal complication risk."

"Development of a virtual spacer to support the decision for the placement of an implantable rectum spacer for prostate cancer radiotherapy: Comparison of dose, toxicity and cost-effectiveness."

"Radiomics: the bridge between medical imaging and personalized medicine"

"Big Data in radiation therapy: challenges and opportunities"

"Modelling the temperature evolution of bone under high intensity focused ultrasound."

$\begin{array}{ll}\begin{array}{l}\text { BioMed Research } \\ \text { International } 2019\end{array} & \text { 1st author } \\ \text { JCO Clin Cancer Inform } & \text { Co-author } \\ \mathbf{2 0 1 9} & \\ \begin{array}{l}\text { Acta Oncol } 2018 \\ \text { 1st author }\end{array} \\ \begin{array}{l}\text { Advances in Particle } \\ \text { Therapy } 2018\end{array} & \text { 1st author } \\ \begin{array}{l}\text { Strahlenther Onkol } \\ \mathbf{2 0 1 8}\end{array} & \text { 2nd author } \\ \end{array}$

Radiother Oncol 2017 1st author

Nat Rev Clin Oncol Co-author 2017

Br J Radiol 2017 Co-author

Phys Med Biol $2016 \quad$ Co-author 
